\title{
Mechanisms and imaging of myocardial injury and repair
}

Citation for published version (APA):

Zandbergen, H. R. (2014). Mechanisms and imaging of myocardial injury and repair. [Doctoral Thesis, Maastricht University]. Datawyse / Universitaire Pers Maastricht. https://doi.org/10.26481/dis.20141223hz

Document status and date:

Published: 01/01/2014

DOI:

10.26481/dis.20141223hz

Document Version:

Publisher's PDF, also known as Version of record

\section{Please check the document version of this publication:}

- A submitted manuscript is the version of the article upon submission and before peer-review. There can be important differences between the submitted version and the official published version of record.

People interested in the research are advised to contact the author for the final version of the publication, or visit the DOI to the publisher's website.

- The final author version and the galley proof are versions of the publication after peer review.

- The final published version features the final layout of the paper including the volume, issue and page numbers.

Link to publication

\footnotetext{
General rights rights.

- You may freely distribute the URL identifying the publication in the public portal. please follow below link for the End User Agreement:

www.umlib.nl/taverne-license

Take down policy

If you believe that this document breaches copyright please contact us at:

repository@maastrichtuniversity.nl

providing details and we will investigate your claim.
}

Copyright and moral rights for the publications made accessible in the public portal are retained by the authors and/or other copyright owners and it is a condition of accessing publications that users recognise and abide by the legal requirements associated with these

- Users may download and print one copy of any publication from the public portal for the purpose of private study or research.

- You may not further distribute the material or use it for any profit-making activity or commercial gain

If the publication is distributed under the terms of Article $25 \mathrm{fa}$ of the Dutch Copyright Act, indicated by the "Taverne" license above, 


\section{Mechanisms and Imaging of Myocardial Injury and Repair}

H.R. Zandbergen

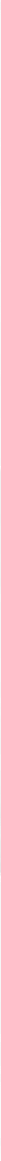


(C) Copyright H.R. Zandbergen, Maastricht 2014

ISBN 9789461593825

Production: Datawyse | Universitaire Pers Maastricht

Cover illustration: The hands of Jurrian and Charlotte-Sophie Zandbergen

Financial support by the Dutch Heart Foundation and Stichting Hartsvrienden RESCAR for the publication of this thesis is gratefully acknowledged

Further financial support granted by Sorin Group Nederland NV, Bard Netherlands BV, QP\&S NV, Olympus Nederland BV, Krijnen Medical Innovations BV, Covidien, St. Jude Medical Nederland BV and Maquet Netherlands BV is gratefully acknowledged 


\title{
Mechanisms and Imaging of Myocardial Injury and Repair
}

\author{
PROEFSCHRIFT \\ ter verkrijging van de graad van doctor aan de Universiteit Maastricht, \\ op gezag van de Rector Magnificus, \\ Prof. Dr. L.L.G. Soete, \\ volgens het besluit van het College van Decanen, \\ in het openbaar te verdedigen, \\ op dinsdag 23 december 2014 om 16.00 uur \\ door \\ Harmen Reinier Zandbergen \\ geboren op 17 oktober 1978 te Deventer
}

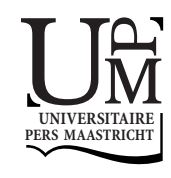


Promotiecommissie

\section{Promotor}

Prof. dr. J.G. Maessen

\section{Copromotores}

Prof. dr. L. Hofstra

Prof. dr. S. Gelsomino (Careggi Hospital, Florence, Italy)

\section{Beoordelingscommissie}

Prof. dr. N. Bouvy (voorzitter)

Prof. dr. H.P. Brunner-la Rocca

Prof. dr. H. ten Cate

Prof. dr. R.P. Koopmans

Prof. dr. M. La Meir (Universitair Ziekenhuis Brussel)

Prof. dr. G. Wesseling 
Aan Marjoes, Jurrian en Charlotte-Sophie Aan mijn ouders 



\section{Table of contents}

Chapter 1 Introduction 9

Chapter 2 Molecular imaging of ventricular remodeling 23

Chapter 3 Macrophage depletion in hypertensive rats accelerates $\quad 41$ development of cardiomyopathy

Chapter 4 Early and late macrophage depletion in acute myocardial infarction 53

Chapter 5 Geometrical models for cardiac mri in rodents: comparison of 59 quantification of left ventricular volumes and function by various geometrical models with a full-volume mri data set in rodents

Chapter 6 Annexin a5 uptake in ischemic myocardium: demonstration of reversible phoshatidylserine externalization and feasibility of radionuclide imaging

Chapter 7 Molecular imaging for efficacy of pharmacologic intervention in myocardial remodeling

Chapter 8 Noninvasive molecular imaging of cell death in myocardial infarction using ${ }^{111}$ in-GSAO

Chapter 9 Discussion

Summary

Samenvatting (Summary in Dutch)

Valorisatie

Dankwoord

Curriculum vitae

Publications 

CHAPTER 1

Introduction 



\section{Cardiovascular disease: still a challenge despite therapeutic revolutions}

Cardiovascular diseases are the leading cause of death both in the Western world and developing countries, and in men and women alike. In 2005, the main cause of death in the USA due to disease of the heart alone was more than of all neoplastic disease combined (1). It is expected that these numbers will continue to increase in upcoming decades due to rising percentages of obesity, hypertension, hyperlipidemia and diabetes as well as population ageing (2). There is, therefore, increased emphasis on preventing atherosclerosis by modifying risk factors, such as healthy nutrition, exercise, and avoidance of smoking tobacco.

Myocardial infarction (MI) is the irreversible necrosis of heart muscle secondary to prolonged ischemia. Each year, more than a million cases of $\mathrm{Ml}$ occur and $>400.000$ people die of coronary artery disease (3). Over the past three decades therapeutic advances such as thrombolytic treatment, angioplasty, coronary bypass surgery, and the use of aspirin, beta-blockers and angiotensin-converting-enzyme (ACE) inhibitors have contributed to reduce mortality after $\mathrm{Ml}$ (4). These improvements were based on 3 important observations. First, it was noticed that the mortality of patients with $\mathrm{MI}$ is closely linked to infarct size and degree of pump function impairment $(5,6)$. Second, studies done on the delay between the onset of symptoms and start of reperfusion therapy consistently found that the time between the onset of coronary occlusion and the benefit of reperfusion therapy is limited to three to four hours (7-9). A third important observation was that following the acute phase of a first infarction, a substantial fraction of patients remain at relatively high risk of subsequent coronary events (10-12). Early angiographic studies in $\mathrm{Ml}$ patients showed that in about $80 \%$ of patients an obstructive coronary thrombus was the main cause for the acute coronary syndrome (13). This finding has generated an era in which patients received thrombolytic therapy, for instance streptokinase or tissue plasminogen activator (tPA) in the acute phase of myocardial infarction (14). However, in the early 1990's it became clear that acute percutaneous intervention ( $\mathrm{PCl})$ provided substantial benefit both in terms of cardiac function as well as mortality, even in the long run $(15,16)$. Therefore, current guidelines recommend early $\mathrm{PCl}$ as the preferred treatment option unless there is no $\mathrm{PCl}$ centre available nearby $(17,18)$.

These improvements apart from better survival, resulted in a decrease in acute complications following $\mathrm{Ml}$ such as acute Congestive Heart Failure (CHF), myocardial rupture and/or arrhythmias (4). In contrast, with more patients surviving the initial stage of $\mathrm{Ml}$, the development of late complications has become a more relevant health care problem $(19,20)$. The development of left ventricular dilatation and loss of pump function in the years following the acute myocardial injury, induced by the formation of the scar, and the impact of the local loss of function on pressure and tensile forces in the rest of the heart, are the subject of intense research. 
Many trials in this area have convincingly shown that inhibition of the renin-angiotensin system, through either ACE inhibitors and/or angiotensin receptor 1 blockers preserve cardiac function and decrease mortality post $\mathrm{MI}$ (21-25). In addition, intervention in mineralo-corticoid signaling has proven to preserve cardiac function and decrease mortality significantly $(26,27)$. Despite these advances, still a substantial fraction, about onethird of MI patients, develop CHF in the long run $(20,28,29)$.

Therefore, there is still a lot to be learned and to be gained from research in cardiac infarct healing and adverse left ventricular remodeling.

\section{Phases of infarct healing, inflammatory cell influx}

In non-injured and normal functioning human heart most of the volume is occupied by cardiomyocytes. The main function of cardiomyocytes is to provide coordinated contractile forces to pump out blood. For this purpose, cardiomyocytes contain specific contractile proteins, such as troponin T (TNT) and troponin I (TNI).

The structure of the heart is provided by the extracellular matrix (ECM), which serves as a scaffold for the contractile cells (30). The extracellular matrix consists of various types of collagen, which, in a normal heart, take up 1-2\% of the total heart volume. These collagens are produced by fibroblasts and in pathologic settings myofibroblasts, which are also found in the cardiac interstitium (31).

Changes in loading conditions have an almost immediate effect on the different cellular and structural components of the hearts. For instance, it is known that cardiomyocytes start to increase their protein synthesis within hours after the onset of increased loading (32). Due to long-standing elevated pressure, cardiomyocytes will start to increase in size, resulting in hypertrophy of the heart (33).

In many ways, wound healing in the heart mimics wound healing in other tissue types, such as skin wound healing (34). However, a set of distinctive characteristics result in marked differences in the final result of cardiac wound healing. First, cardiomyocytes are terminally, or almost terminally differentiated cells, which allows for only limited regenerative capacity.

In general, 4 distinctive phase in cardiac wound healing can be recognized $(34,35)$. In the first phase, which lasts 1-2 days, cardiomyocyte cell death occurs, either trough apoptotic or necrotic cell death, resulting in substantial cell debris. The second phase is the acute inflammation phase, and overlaps quite extensively with the first phase, since it starts already after 6 hours, and continues to up to 5-6 days. In early phase 2, degradation of extracellular matrix occurs, which enable migration of invading Polymorphonuclear Leukocytes (PMN's), and later on, monocytes/macrophages. Depletion of macrophages in models of myocardial infarction, as opposed to depletion of PMNs, results in prolonged presence of cell debris in the cardiac wound, indicating that these are the main phagocytic cells (36). The third phase of cardiac wound healing is characterized by 
the deposition of novel matrix proteins, including fibrin, and collagen type III, and later collagen type I (31). It is thought that most of the new ECM production is due to the activity of myofibroblasts (31). In this phase the scar is still a cell rich tissue, which resembles granulation tissue as found in outer tissue wounds, such as the skin. Another essential process that occurs in the third phase is the formation of novel vessels, also called angiogenesis. This process has generated a lot of attention in recent years, since it is thought that increased blood supply to the cardiac wound may help to preserve cardiac function, and to limit the final injury (37).

In the fourth phase of cardiac wound healing, most cell types, such as the macrophages, start to disappear from the wound. Most likely, this is the consequence of apoptotic cell death of these phagocytic cells (38). By contrast, a large part of the myofibroblasts remains present in the scar area (39). Another essential process is the crosslinking of collagen, which is required to provide sufficient strength to withstand the pressure and tensile forces to which the scar is continuously being exposed to (40).

\section{Adverse remodeling}

The repair mechanisms described above are essential for maintaining the functional and structural integrity of the heart. In addition, several compensatory processes are initiated in the non-infarcted area. Although potentially beneficial, exaggerated occurrence of these processes can lead to morphological and functional changes to the heart which eventually lead to declining pumping capacity and the development of heart failure. The complex of these changes is known as adverse remodeling.

The presence of an infarcted area in the heart and the resulting changes in loading conditions trigger changes in the extra cellular matrix in the remote myocardium. In the normal heart, collagens in the ECM account for $1-2 \%$ of the entire cardiac volume. However, following acute myocardial infarction, this percentage can easily double. The pattern of enhanced collagen deposition in the remote parts of the heart is to a large extent similar to that of the infarct itself (31). First, collagen type III is deposited in the ECM, followed by collagen type I. The effect of the increased deposition in the remote areas is probably to provide enhanced strength and to prevent dilatation. However, the downside of the increase in ECM content is enhanced stiffness, which may result in resistance to diastolic filling and eventually diastolic failure (41).

Another change in the non-infarcted myocardium is cardiomyocyte hypertrophy (42), which is triggered to compensate for the loss of cardiac muscle. However, exaggerated hypertrophy has several unfavorable effects. It may reduce the ventricular cavity volume, thereby reduce myocardial filling and contribute to development of diastolic heart failure (43). Moreover, cardiomyocyte hypertrophy decreases the capillary to myocyte-fiber ratio, since endothelial cell proliferation cannot fully compensate for the 
growth of the cardiomyocyte. The consequence is an increase in oxygen diffusion distance and, in the end a relative oxygen deficit (44).

One of the key regulators of fibrosis and cardiomyocyte hypertrophy is the reninangiotensin-aldosterone system (RAAS) $(45,46)$; inhibition of the RAS-pathway has shown to effectively reduce adverse remodeling through inhibition of cellular hypertrophy and fibrosis $(21-25,27)$. It is well established that myocardial neurohumoral upregulation more so than the systemic upregulation contributes to ventricular remodeling and development of post-MI-CHF (47). In particular, angiotensin II type 1 receptor expression within the myocardium is associated with interstitial fibrosis and ventricular dysfunction (48). Transgenic mice lacking angiotensin II type 1 receptor expression exhibit minimal geometric and structural remodeling following $\mathrm{MI}$, and lower upregulation of so-called fetal genes, such as TGF- $\beta 1$, and collagen deposition in comparison to the wild-type animals (49).

Angiogenesis represents the formation of new capillaries by cellular outgrowth from existing microvessels and occurs as part of the natural healing process following injury. The physiological importance of controlling neo-angiogenesis on remodeling following $\mathrm{Ml}$ is less clear. Experimental studies aimed to induce angiogenesis following $\mathrm{Ml}$ have shown beneficial effects on LV function; however, this has not been proven in clinical studies so far (50).

Another phenomenon involved of cardiac remodeling resulting in congestive heart failure in late stages following $\mathrm{MI}$, is the development of progressive left ventricular dilatation, leading to loss of pump function of the heart (42). The loss of cardiomyocytes through apoptosis may contribute to this phenomenon. Several preclinical and clinical studies have clearly demonstrated that apoptosis occurs in the remote areas of the left ventricular wall (51-53). Although the rate of apoptosis is small, the idea is that this slow but gradual loss of cells eventually contributes to decrease in cardiac performance. The renin-angiotensin-system is also involved in this process $(54,55)$. Indeed, inhibition of AT1 receptor activity has shown to be beneficial in the prevention of cell loss through apoptosis $(48,56,57)$.

\section{Possible role of the macrophage in infarct healing and fibrosis}

As mentioned before, the macrophage is the principal phagocyte in the myocardial scar following infarction. Depletion of macrophages has shown to result in decreased clearance of dead cells and persistence of cell debris up to $3-4$ weeks $(58,59)$. In skin wound healing, injection of macrophages accelerates wound healing, including the clearance of cell debris, and the formation of collagen. Given the essential difference between cardiac wound healing and skin wound healing, being the lack of significant regenerative capacity in the heart and the exposure of the cardiac scar to repetitive pressure and 
tensile forces, it is not altogether clear whether enhanced presences of macrophages will be beneficial or detrimental $(59,60)$.

In a recent study, performed in a cryo-injury model in the mouse, it was shown that depletion of macrophages through the administration of clodronate resulted in decreased wound healing, as evidenced by persistence of cell debris, formation of a scar with low collagen content, a decrease in the presence of myofibroblasts and less angiogenesis (61). Furthermore, this study observed that cardiac cryo-injury in the absence of macrophages resulted in an increase in left ventricular size as compared to controls. From these data, it was concluded that macrophage depletion impairs wound healing and increases let ventricular remodeling. However, from the study data it is apparent that in the control cryo-injury group no remodeling of the left ventricle occurred during the course of the study, which is in contrast to observations in MI models, which consistently show substantial dilatation and adverse remodeling. Therefore, the cryo-injury model does not necessarily reflect the events during myocardial wound healing during myocardial infarction. In addition, the cryo-injury study did not provide functional data on the effect of macrophage depletion on pump function of the left ventricle.

Other studies have attempted to enhance the presence of macrophages in a rat model $\mathrm{Ml}$ through the administration of macrophage colony stimulating factor ( $\mathrm{M}$ CSF)(62). The administration of M-CSF resulted in an increase in macrophage presence, and enhanced deposition of collagen type I and III. In addition, left ventricular function was increased in the $\mathrm{MI}$ group treated with Macrophage Colony Stimulating Factor (M$\mathrm{CSF}$ ) compared to $\mathrm{MI}$ control, although these changes were not significant. This finding is in contrast to other preclinical studies, which have attempted to suppress collagen deposition. For example, in the before mentioned study performed in AT-1 receptor KO mice it was shown that prevention of collagen deposition and was associated with preservation of cardiac function, and prevention of cardiac remodeling (49). Similarly, prevention of cardiac fibrosis through interference in mineralcorticoid signaling has shown to prevent fibrosis and preserve cardiac function $(27,63)$. Therefore, the improved function in the treated animals most likely has other causes than accelerated wound healing and enhanced deposition of collagen. Another argument that speaks in favor of inhibition of fibrosis rather than its promotion is the fact that cardiac regeneration therapy is probably easier to achieve in an area devoid of a dense and fibrotic scar. The association between macrophages and cardiac fibrosis is suggested further by observation in mouse embryo's, which only then shows fibrosis in wounds when macrophages are recruited to the wound area (64). Given the studies in the macrophage depletion model and the M-CSF stimulation studies, it is likely that macrophages are important mediators in the fibrotic process. The role of the macrophage in cardiac wound healing may in large part depend on the balance between certain signaling molecules secreted by macrophages (36,65-67). In a macrophage specific overexpression of urokinase plasminogen activator in a mouse model it was noted that enhanced cardiac fibrosis took place, even in the absence of significant epicardial disease. This same study 
showed that the enhanced fibrosis was specific for the heart, since other organs seemed resistant to macrophage infiltration, and subsequent fibrosis.

\section{Aim of the thesis}

The aims of the thesis are twofold. In the first part, we investigated the role of the macrophage in ventricular remodeling following acute myocardial infarction, to better understand the critical influence of this cell type in infarct healing post myocardial infarction. In the second part, we have used molecular imaging techniques to better understand the different phases of myocardial infarct injury following ischemia. The aims are described in details below.

\section{(1): To investigate the role of macrophages in MI and other experimental models}

As suggested from the literature discussed above, macrophages potentially play an important role in cardiac fibrosis. However, a number of questions remain unanswered. The main difficulty with the macrophage depletion study in a cryo-injury model (61) in the heart is the absence of ventricular remodeling in the presence of macrophages. In addition, functional analysis was not performed in that study. Therefore, the question remains to which extent macrophage depletion would affect cardiac function and structure in models that resemble acute myocardial injury in the setting of severe ischemia better. In addition, it remains to be seen whether macrophage depletion affects cardiac function and ECM structure in other models of cardiac failure, such as hypertension heart disease.

Based on these questions we set out to perform macrophage depletion experiments in different models of cardiac disease. In addition, we studied the effect of inhibition of macrophage migration in CCR2 KO mice, subjected to myocardial infarction. Furthermore, we aimed to investigate the impact of short ischemia episodes on cardiomyocyte recovery, and micro repair in its direct environment.

\section{(2): To use Biomarkers and Molecular Imaging Probes for Cell Death Assessment}

Cell death is an essential biological process that plays a vital physiological and pathological role within an organism. Programmed cell death is critical for the correct formation of organs and tissues during development and also functions to rid the body of cells that have been infected or damaged by pathogens $(68,69)$.

There are several currently validated cell death biomarkers for molecular imaging. Most cell death imaging studies employ one of three modalities: optical, radionuclear, or magnetic resonance imaging. These modalities have complementary properties that make them suitable for different types of in vivo imaging applications 
(70-73). In comparison to the other methods, radionuclear imaging methods such as Positron Emission Tomography (PET) and Single Photon Emission Computed Tomography (SPECT) provide much deeper tissue penetration and greatly reduced scattering of the emitted signal, and thus, are more attractive for many types of clinical applications. In $\mathrm{Ml}$ and heart failure three biological features represents attractive targets for molecular imaging: 1) apoptosis 2) the renin-angiotensin pathway and 3) the biological processes of angiogenesis and fibrogenesis.

First, we employed labeled annexin-A5 (AA5) for radionuclide imaging to demonstrate the reversible phosphatidylserine externalization in cardiomyocyte apoptosis. In addition, We exploited the capacity of a radiolabeled trivalent arsenical peptide, 4-(N-(Sglutathionylacetyl)amino) phenylarsonous acid (GSAO) to target intracellular dithiol molecules like Heat shock protein 90 (HSP90) to identify cell death. Finally, using molecular imaging techniques, we examined interstitial alterations during Post-MI remodeling and assessed the efficacy of antiangiotensin and antimineralocorticoid intervention, alone and in combination employing radionuclide imaging with a novel arginineglutamate-aspartate (RGD) peptide, technetium-99m-labeled Cy5.5-RGD-imaging peptide (CRIP). 


\section{References}

1. Kung HC, Hoyert DL, Xu J, Murphy SL. Deaths: final data for 2005. Natl Vital Stat Rep 2008 Apr 24;56(10):1-120.

2. Heidenreich PA, Trogdon JG, Khavjou OA, Butler J, Dracup K, Ezekowitz MD, et al. Forecasting the future of cardiovascular disease in the United States: a policy statement from the American Heart Association. Circulation 2011 Mar 1;123(8):933-944.

3. Go AS, Mozaffarian D, Roger VL, Benjamin EJ, Berry JD, Borden WB, et al. Heart disease and stroke statistics--2013 update: a report from the American Heart Association. Circulation 2013 Jan 1;127(1):e6-e245.

4. White HD, Chew DP. Acute myocardial infarction. Lancet 2008 Aug 16;372(9638):570-584.

5. Geltman EM, Ehsani AA, Campbell MK, Schechtman K, Roberts R, Sobel BE. The influence of location and extent of myocardial infarction on long-term ventricular dysrhythmia and mortality. Circulation 1979 Oct;60(4):805-814.

6. Sobel BE, Bresnahan GF, Shell WE, Yoder RD. Estimation of infarct size in man and its relation to prognosis. Circulation 1972 Oct;46(4):640-648.

7. Reimer KA, Lowe JE, Rasmussen MM, Jennings RB. The wavefront phenomenon of ischemic cell death. 1. Myocardial infarct size vs duration of coronary occlusion in dogs. Circulation 1977 Nov;56(5):786-794.

8. Brodie BR, Stuckey TD, Wall TC, Kissling G, Hansen CJ, Muncy DB, et al. Importance of time to reperfusion for 30-day and late survival and recovery of left ventricular function after primary angioplasty for acute myocardial infarction. J Am Coll Cardiol 1998 Nov;32(5):1312-1319.

9. Berger PB, Bell MR, Holmes DR,Jr, Gersh BJ, Hopfenspirger M, Gibbons R. Time to reperfusion with direct coronary angioplasty and thrombolytic therapy in acute myocardial infarction. Am J Cardiol 1994 Feb $1 ; 73(4): 231-236$.

10. Wong ND, Cupples LA, Ostfeld AM, Levy D, Kannel WB. Risk factors for long-term coronary prognosis after initial myocardial infarction: the Framingham Study. Am J Epidemiol 1989 Sep;130(3):469-480.

11. Wong ND, Levy D, Kannel WB. Prognostic significance of the electrocardiogram after $Q$ wave myocardial infarction. The Framingham Study. Circulation 1990 Mar;81(3):780-789.

12. Wong ND, Wilson PW, Kannel WB. Serum cholesterol as a prognostic factor after myocardial infarction: the Framingham Study. Ann Intern Med 1991 Nov 1;115(9):687-693.

13. Rentrop KP. Thrombi in acute coronary syndromes : revisited and revised. Circulation 2000 Apr 4;101(13):1619-1626.

14. Van de Werf F, Ludbrook PA, Bergmann SR, Tiefenbrunn AJ, Fox KA, de Geest H, et al. Coronary thrombolysis with tissue-type plasminogen activator in patients with evolving myocardial infarction. N Engl J Med 1984 Mar 8;310(10):609-613.

15. Zijlstra F, de Boer MJ, Hoorntje JC, Reiffers S, Reiber JH, Suryapranata H. A comparison of immediate coronary angioplasty with intravenous streptokinase in acute myocardial infarction. N Engl J Med 1993 Mar 11;328(10):680-684.

16. Grines CL, Browne KF, Marco J, Rothbaum D, Stone GW, O'Keefe J, et al. A comparison of immediate angioplasty with thrombolytic therapy for acute myocardial infarction. The Primary Angioplasty in Myocardial Infarction Study Group. N Engl J Med 1993 Mar 11;328(10):673-679.

17. Task Force on the management of ST-segment elevation acute myocardial infarction of the European Society of Cardiology (ESC), Steg PG, James SK, Atar D, Badano LP, Blomstrom-Lundqvist C, et al. ESC Guidelines for the management of acute myocardial infarction in patients presenting with ST-segment elevation. Eur Heart J 2012 Oct;33(20):2569-2619.

18. Anderson JL, Adams CD, Antman EM, Bridges CR, Califf RM, Casey DE,Jr, et al. 2012 ACCF/AHA focused update incorporated into the ACCF/AHA 2007 guidelines for the management of patients with unstable angina/non-ST-elevation myocardial infarction: a report of the American College of Cardiology Foundation/American Heart Association Task Force on Practice Guidelines. J Am Coll Cardiol 2013 Jun 11;61(23):e179-347.

19. Jhund PS, McMurray JJ. Heart failure after acute myocardial infarction: a lost battle in the war on heart failure? Circulation 2008 Nov 11;118(20):2019-2021. 
20. Velagaleti RS, Pencina MJ, Murabito JM, Wang TJ, Parikh NI, D'Agostino RB, et al. Long-term trends in the incidence of heart failure after myocardial infarction. Circulation 2008 Nov 11;118(20):2057-2062.

21. Zuanetti G, Latini R, Maggioni AP, Franzosi M, Santoro L, Tognoni G. Effect of the ACE inhibitor lisinopril on mortality in diabetic patients with acute myocardial infarction: data from the GISSI-3 study. Circulation 1997 Dec 16;96(12):4239-4245.

22. Latini R, Maggioni AP, Flather M, Sleight $P$, Tognoni G. ACE inhibitor use in patients with myocardial infarction. Summary of evidence from clinical trials. Circulation 1995 Nov 15;92(10):3132-3137.

23. Yusuf S, Pepine CJ, Garces C, Pouleur H, Salem D, Kostis J, et al. Effect of enalapril on myocardial infarction and unstable angina in patients with low ejection fractions. Lancet 1992 Nov 14;340(8829):11731178.

24. Fox KM, EURopean trial On reduction of cardiac events with Perindopril in stable coronary Artery disease Investigators. Efficacy of perindopril in reduction of cardiovascular events among patients with stable coronary artery disease: randomised, double-blind, placebo-controlled, multicentre trial (the EUROPA study). Lancet 2003 Sep 6;362(9386):782-788.

25. Verma S, Strauss M. Angiotensin receptor blockers and myocardial infarction. BMJ 2004 Nov 27;329(7477):1248-1249.

26. Hayashi M, Tsutamoto T, Wada A, Tsutsui T, Ishii C, Ohno K, et al. Immediate administration of mineralocorticoid receptor antagonist spironolactone prevents post-infarct left ventricular remodeling associated with suppression of a marker of myocardial collagen synthesis in patients with first anterior acute myocardial infarction. Circulation 2003 May 27;107(20):2559-2565.

27. Pitt B, Remme W, Zannad F, Neaton J, Martinez F, Roniker B, et al. Eplerenone, a selective aldosterone blocker, in patients with left ventricular dysfunction after myocardial infarction. N Engl J Med 2003 Apr 3;348(14):1309-1321.

28. Hellermann JP, Jacobsen SJ, Redfield MM, Reeder GS, Weston SA, Roger VL. Heart failure after myocardial infarction: clinical presentation and survival. Eur J Heart Fail 2005 Jan;7(1):119-125.

29. Spencer FA, Meyer TE, Goldberg RJ, Yarzebski J, Hatton M, Lessard D, et al. Twenty year trends (19751995) in the incidence, in-hospital and long-term death rates associated with heart failure complicating acute myocardial infarction: a community-wide perspective. J Am Coll Cardiol 1999 Nov 1;34(5):13781387.

30. Weber KT. Cardiac interstitium in health and disease: the fibrillar collagen network. J Am Coll Cardiol 1989 Jun;13(7):1637-1652.

31. van den Borne SW, Diez J, Blankesteijn WM, Verjans J, Hofstra L, Narula J. Myocardial remodeling after infarction: the role of myofibroblasts. Nat Rev Cardiol 2010 Jan;7(1):30-37.

32. Sadoshima J, Izumo S. Mechanical stretch rapidly activates multiple signal transduction pathways in cardiac myocytes: potential involvement of an autocrine/paracrine mechanism. EMBO J 1993 Apr;12(4):1681-1692.

33. Kerkela R, Force T. Recent insights into cardiac hypertrophy and left ventricular remodeling. Curr Heart Fail Rep 2006 Apr;3(1):14-18.

34. Ertl G, Frantz S. Healing after myocardial infarction. Cardiovasc Res 2005 Apr 1;66(1):22-32.

35. Weber KT, Sun Y, Katwa LC. Wound healing following myocardial infarction. Clin Cardiol 1996 Jun;19(6):447-455.

36. Lambert JM, Lopez EF, Lindsey ML. Macrophage roles following myocardial infarction. Int J Cardiol 2008 Nov 12;130(2):147-158.

37. Lee SH, Wolf PL, Escudero R, Deutsch R, Jamieson SW, Thistlethwaite PA. Early expression of angiogenesis factors in acute myocardial ischemia and infarction. N Engl J Med 2000 Mar 2;342(9):626-633.

38. Rafatian N, Westcott KV, White RA, Leenen FH. Cardiac Macrophages and Apoptosis after Myocardial Infarction: Effects of Central MR Blockade. Am J Physiol Regul Integr Comp Physiol 2014 Aug 6.

39. Turner NA, Porter KE. Function and fate of myofibroblasts after myocardial infarction. Fibrogenesis Tissue Repair 2013 Mar 1;6(1):5-1536-6-5. 
40. Woodiwiss AJ, Tsotetsi OJ, Sprott S, Lancaster EJ, Mela T, Chung ES, et al. Reduction in myocardial collagen cross-linking parallels left ventricular dilatation in rat models of systolic chamber dysfunction. Circulation 2001 Jan 2;103(1):155-160.

41. Bujak M, Frangogiannis NG. The role of TGF-beta signaling in myocardial infarction and cardiac remodeling. Cardiovasc Res 2007 May 1;74(2):184-195.

42. Sutton MG, Sharpe N. Left ventricular remodeling after myocardial infarction: pathophysiology and therapy. Circulation 2000 Jun 27;101(25):2981-2988.

43. Pfeffer MA, Braunwald E. Ventricular remodeling after myocardial infarction. Experimental observations and clinical implications. Circulation 1990 Apr;81(4):1161-1172.

44. Otterstad JE. Ischaemia and left ventricular hypertrophy. Eur Heart J 1993 Oct;14 Suppl F:2-6.

45. Dzau VJ, Colucci WS, Hollenberg NK, Williams GH. Relation of the renin-angiotensin-aldosterone system to clinical state in congestive heart failure. Circulation 1981 Mar;63(3):645-651.

46. Weber KT. Aldosterone in congestive heart failure. N Engl J Med 2001;345(23):1689-1697.

47. Dzau VJ. Tissue renin-angiotensin system in myocardial hypertrophy and failure. Arch Intern Med 1993 Apr 26;153(8):937-942.

48. Rosenkranz S. TGF-beta1 and angiotensin networking in cardiac remodeling. Cardiovasc Res 2004 Aug 15;63(3):423-432.

49. Harada K, Sugaya T, Murakami K, Yazaki Y, Komuro I. Angiotensin II type 1A receptor knockout mice display less left ventricular remodeling and improved survival after myocardial infarction. Circulation 1999 Nov 16;100(20):2093-2099.

50. Atluri P, Woo YJ. Pro-angiogenic cytokines as cardiovascular therapeutics: assessing the potential. BioDrugs 2008;22(4):209-222.

51. Olivetti G, Abbi R, Quaini F, Kajstura J, Cheng W, Nitahara JA, et al. Apoptosis in the failing human heart. N Engl J Med 1997;336(16):1131-1141.

52. Sam F, Sawyer DB, Chang DL, Eberli FR, Ngoy S, Jain M, et al. Progressive left ventricular remodeling and apoptosis late after myocardial infarction in mouse heart. Am J Physiol Heart Circ Physiol 2000 Jul;279(1):H422-8.

53. Narula J, Haider N, Arbustini E, Chandrashekhar Y. Mechanisms of disease: apoptosis in heart failureseeing hope in death. Nature Clinical Practice Cardiovascular Medicine 2006;3(12):681-688.

54. Kajstura J, Cigola E, Malhotra A, Li P, Cheng W, Meggs LG, et al. Angiotensin II Induces Apoptosis of Adult Ventricular Myocytes< i> In Vitro</i>. J Mol Cell Cardiol 1997;29(3):859-870.

55. Diez J, Panizo A, Hernandez M, Vega F, Sola I, Fortuno MA, et al. Cardiomyocyte apoptosis and cardiac angiotensin-converting enzyme in spontaneously hypertensive rats. Hypertension 1997 Nov;30(5):10291034.

56. Takemoto M, Egashira K, Tomita H, Usui M, Okamoto H, Kitabatake A, et al. Chronic angiotensinconverting enzyme inhibition and angiotensin II type 1 receptor blockade: effects on cardiovascular remodeling in rats induced by the long-term blockade of nitric oxide synthesis. Hypertension 1997 Dec;30(6):1621-1627.

57. Gajarsa JJ, Kloner RA. Left ventricular remodeling in the post-infarction heart: a review of cellular, molecular mechanisms, and therapeutic modalities. Heart Fail Rev 2011;16(1):13-21.

58. Nahrendorf M, Pittet MJ, Swirski FK. Monocytes: protagonists of infarct inflammation and repair after myocardial infarction. Circulation 2010 Jun 8;121(22):2437-2445.

59. Frantz S, Hofmann U, Fraccarollo D, Schafer A, Kranepuhl S, Hagedorn I, et al. Monocytes/macrophages prevent healing defects and left ventricular thrombus formation after myocardial infarction. FASEB J 2013 Mar;27(3):871-881.

60. Swirski FK, Nahrendorf M. Leukocyte behavior in atherosclerosis, myocardial infarction, and heart failure. Science 2013 Jan 11;339(6116):161-166.

61. van Amerongen MJ, Harmsen MC, van Rooijen N, Petersen AH, van Luyn MJ. Macrophage depletion impairs wound healing and increases left ventricular remodeling after myocardial injury in mice. The American journal of pathology 2007;170(3):818-829. 
62. Shim W, Mehta A, Lim SY, Zhang G, Lim CH, Chua T, et al. G-CSF for stem cell therapy in acute myocardial infarction: friend or foe? Cardiovasc Res 2011 Jan 1;89(1):20-30.

63. Silvestre JS, Heymes C, Oubenaissa A, Robert V, Aupetit-Faisant B, Carayon A, et al. Activation of cardiac aldosterone production in rat myocardial infarction: effect of angiotensin II receptor blockade and role in cardiac fibrosis. Circulation 1999 May 25;99(20):2694-2701.

64. Hopkinson-Woolley J, Hughes D, Gordon S, Martin P. Macrophage recruitment during limb development and wound healing in the embryonic and foetal mouse. J Cell Sci 1994 May;107 ( Pt 5)(Pt 5):1159-1167.

65. Dobaczewski $M$, Chen $W$, Frangogiannis NG. Transforming growth factor (TGF)- $\beta$ signaling in cardiac remodeling. J Mol Cell Cardiol 2011;51(4):600-606.

66. GUILLfiN I, Blanes M, GOMEZ-LECHON M, CASTELL J. Cytokine signaling during myocardial infarction: sequential appearance of ILAp and IL-6. 1995.

67. Neumann FJ, Ott I, Gawaz M, Richardt G, Holzapfel H, Jochum M, et al. Cardiac release of cytokines and inflammatory responses in acute myocardial infarction. Circulation 1995 Aug 15;92(4):748-755.

68. Renehan AG, Booth C, Potten CS. What is apoptosis, and why is it important? BMJ 2001 Jun 23;322(7301):1536-1538.

69. Elmore S. Apoptosis: a review of programmed cell death. Toxicol Pathol 2007 Jun;35(4):495-516.

70. De Saint-Hubert M, Prinsen K, Mortelmans L, Verbruggen A, Mottaghy FM. Molecular imaging of cell death. Methods 2009;48(2):178-187.

71. Berry NC, Sosnovik DE. Cardiomyocyte Death: Insights from Molecular and Microstructural Magnetic Resonance Imaging. Pediatr Cardiol 2011;32(3):290-296.

72. Weissleder R, Mahmood U. Molecular Imaging 1. Radiology 2001;219(2):316-333.

73. Shah K, Weissleder R. Molecular optical imaging: applications leading to the development of present day therapeutics. NeuroRx 2005;2(2):215-225. 



\section{CHAPTER 2}

\section{Molecular imaging of ventricular remodeling}

H.R. Zandbergen and M.W.M. Schellings

Journal of Nuclear Cardiology, May 2009 



\section{Introduction}

Cardiovascular diseases remain the number one cause of morbidity and mortality, both in the Western world and developing countries and in men and women alike. ${ }^{1}$ In 2005 , the main cause of death in the USA due to disease of the heart alone was more than of all neoplastic disease combined. It is expected that these numbers will continue to increase in the coming decades due to escalating proportions of obesity and the aging population. In addition to cardiac disease, cerebrovascular disease, diabetes, and hypertension result in substantial morbidity and mortality. Therefore, cardiovascular diseases as a whole are killer number one in the Western world, and will most likely remain to be so due to adverse lifestyle changes, including unhealthy diets and lack of exercise.

Myocardial infarction (MI) is the number one cardiac disease and often strikes the individual unexpectedly; in $50 \%$ of cases $\mathrm{MI}$ is the first symptom of coronary atherosclerosis. Atherosclerosis is characterized by a chronic inflammatory response resulting in the formation of multiple plaques in the lumen of the artery. This can happen gradually as a result of progressive plaque growth or suddenly as a result of plaque rupture and, subsequently, thrombosis causing acute MI (AMI).

The improvements in treatment of AMI have resulted in better survival and a decrease in the acute complications of $\mathrm{MI}$, such as acute congestive heart failure (CHF), myocardial rupture, arrhythmias, and conduction system disorders. However, with more patients surviving the initial stage of AMI, the development of late complications of AMI become a more prominent health care problem.

The development of left ventricular dilatation and loss of pump function in the years following the acute myocardial injury, induced by the formation of the scar, and the impact of the local loss of function on pressure and tensile forces in the noninfarcted left ventricle, are the subject of intense research. Many trials in this area have convincingly shown that inhibition of the renin-angiotensin system, through either ACE inhibitors and/or angiotensin receptor 1 blockers, preserve cardiac function and decrease mortality post $\mathrm{MI}^{2}$ In addition, intervention in mineralocorticoid signaling has proven to preserve cardiac function and decrease mortality significantly. ${ }^{3,4}$ Despite these advances, still a substantial fraction, about one-third of AMI patients, will develop pump function disorders of the left ventricle in the long run. The outcome of a relatively recent biomarker known as NT-pro Brain Natriuretic Peptide (NT pro-BNP), which is released by cardiomyocytes after the occurrence of ventricular malfunction, has proven its usefulness in diagnosing heart failure. ${ }^{5}$ Nevertheless, it is hard to predict in which individual patient CHF will occur. Therefore, there is still a lot to be learned and to be gained from research in cardiac infarct healing and adverse left ventricular remodeling.

An upcoming diagnostic tool in analyzing the risk of cardiovascular disease in patients is cardiac imaging. ${ }^{6}$ The capability to visualize macroscopic cardiovascular structures and the anatomical and functional consequences of cardiac diseases in patients has made a remarkable progression in the last decades. The development of coronary 
angiography (CAG), echocardiography, magnetic resonance imaging (MRI), and multidetector CT (MDCT) has improved our approach in diagnosing cardiovascular disease such as atherosclerosis or left ventricular function. However, most of these imaging technologies are able to diagnose the end stage of the disease, rather than the beginning of the disease or even pre-disease states. The next frontier in imaging will be the development of the capability to image fundamental biological or molecular changes which cause cardiovascular disease and are able to predict disease outcome at an early stage. For this purpose, imaging tools other than those mentioned above have to be developed. ${ }^{7}$

Imaging techniques, which visualize the fundamental biological characteristics resulting in cardiovascular disease, may provide the potential to predict cardiovascular catastrophe as an early diagnostic tool. ${ }^{8}$ With the introduction of molecular imaging, the opportunities to detect changes in biology of infarcted hearts and, therefore, cardiac remodeling in vivo have increased significantly in the past decade.

It can be hypothesized that the ability to visualize interstitial processes on a molecular level, which precede the geometric and functional deterioration of the left ventricle, should help to better predict the likelihood and rate of remodeling and development of $\mathrm{HF}^{9}{ }^{9}$ Several key biological features provide attractive targets for molecular imaging in heart failure. First, the development of imaging techniques visualizing the biological events of angiogenesis and fibrogenesis, which are considered key processes in myocardial scar formation and left ventricular remodeling, may provide attractive targets for the identification of patients at risk to develop a failing heart. The use of agents targeted to the integrin alpha $v$ beta 3 (avB3) may offer diagnostic means to achieve this goal.

Second, apoptosis, a form of programmed cell death (PCD), has shown to play a key role in the process of gradual loss of pump function in animal models of heart failure, caused by different triggers. Therefore, imaging apoptosis may provide a means to identify hearts that are in the process of substantial cardiomyocyte loss and subsequent loss of pump function. The use of radiolabeled annexin-A5, which shows strong affinity to apoptotic cells which have externalized phosphatidyl serine, may provide an opportunity to develop such a heart failure imaging diagnostic test.

Finally, it is well known that the activation of the renin-angiotensin axis plays a pivotal role in the development of heart failure post AMI. Therefore, imaging of the different components of renin-angiotensin neurohumoral axis may give an additional diagnostic tool for better identification of patients prone to develop heart failure. This brief review will discuss the recent progresses made in molecular cardiac imaging, focusing mainly on the achievements made in avB3 imaging, apoptosis imaging, and imaging of the activation of the renin-angiotensin system. 


\section{avB3 imaging as a tool to identify post MI remodeling}

As described above, early diagnosis of the impact of the infarct on adverse left ventricular remodeling in the individual patient could prevent worsening of left ventricular pump function by adapting treatment to the individual needs and risks. Two main events that occur in the infarcted area are angiogenesis and collagen deposition. The formation of new blood vessels is crucial for infarct healing, and is induced by several factors, such as basic fibroblast growth factor (bFGF) and vascular endothelial growth factor (VEGF). ${ }^{10}$ Among other regulators of angiogenesis, the avB3 integrin has been identified as a critical angiogenic modulator. Angiogenic vessels display increased expression of this critical integrin. ${ }^{11}$ This discovery led to the development of imaging tools to detect tumor angiogenesis with the use of avB3 integrin targeting agents. ${ }^{12,13}$

In addition, imaging of avB3 integrin has been used to noninvasively visualize angiogenesis in infarcted hearts. Meoli et al used indium-111-RP748 ( ${ }^{111}$ In-RP748), a radiolabeled avB3 targeted compound, to image angiogenesis in infarcted canine hearts with SPECT imaging. ${ }^{14}{ }^{111}$ In-RP748 uptake peaked at 1 week after reperfusion and was associated with avB3 integrin expression on endothelial and vascular smooth muscle cells. In addition, they used the same targeting agent in infarcted dog hearts which showed uptake of ${ }^{111}$ In-RP748 at the side of the perfusion defect using SPECT imaging (Figure 1).
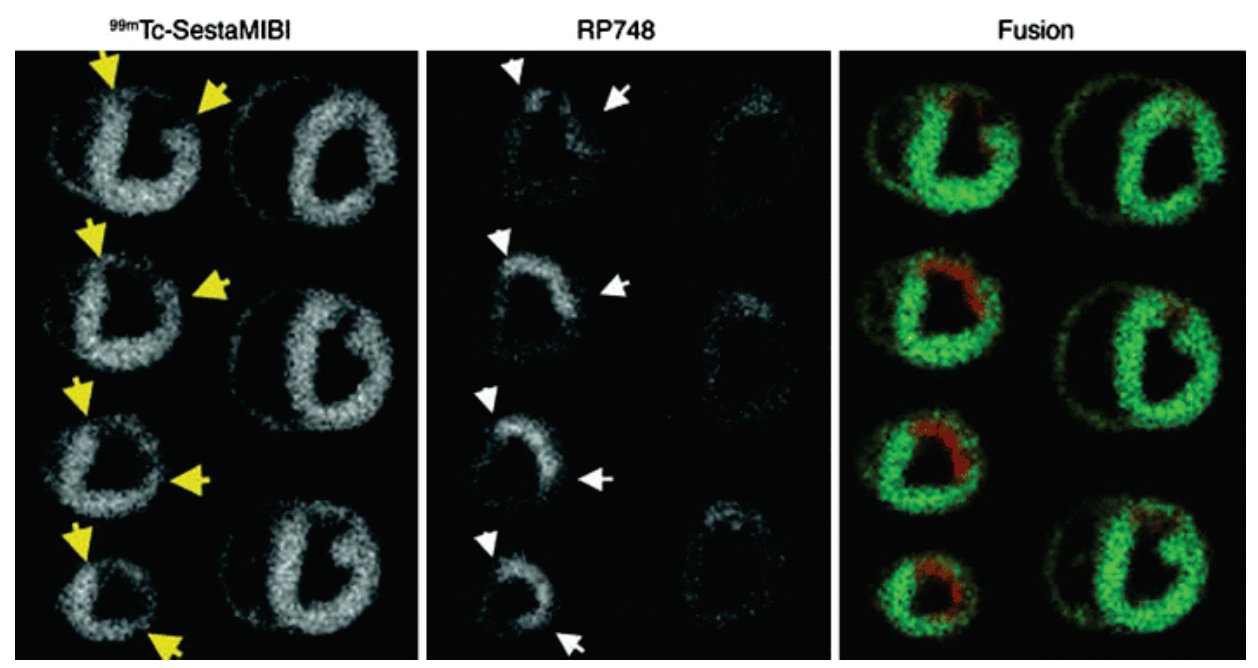

Figure 1. Short axis imaging data 3 weeks after induction of LAD infarction in a dog model. At the site of the perfusion defect (left panel), uptake of the indium-labeled avB3 RP 748 targeting tracer was observed (middle panel). Right panel shows the fusion data.

Recently, ${ }^{18}$ F-Galacto-RGD, a PET tracer targeting specifically avB3 integrin expression, has been introduced. ${ }^{15}$ Higuchi et al used this tracer to monitor avB3 integrin expression in rat hearts after ischemia/reperfusion, and reported results comparable with ${ }^{111} \mathrm{In}$ - 
RP748 imaging. ${ }^{16}$ Clinical use of the ${ }^{18}$ F-Galacto-RGD PET tracer in a patient with $\mathrm{MI}$ was reported shortly. ${ }^{17}$

Apart from its crucial involvement in angiogenesis, avB3 integrin also has been linked to the development of fibrosis, ${ }^{18,19}$ which led our group to evaluate whether avB3 integrin imaging could be used to visualize fibrogenesis in the infarcted heart. ${ }^{20} \mathrm{MI}$ was induced in mice, which received a ${ }^{99 \mathrm{~m}}$ Tc-labeled Cy5.5-RGD imaging peptide (CRIP) intravenously for microSPECT imaging of integrin at 2, 4, and 12 weeks after MI (Figure 2).
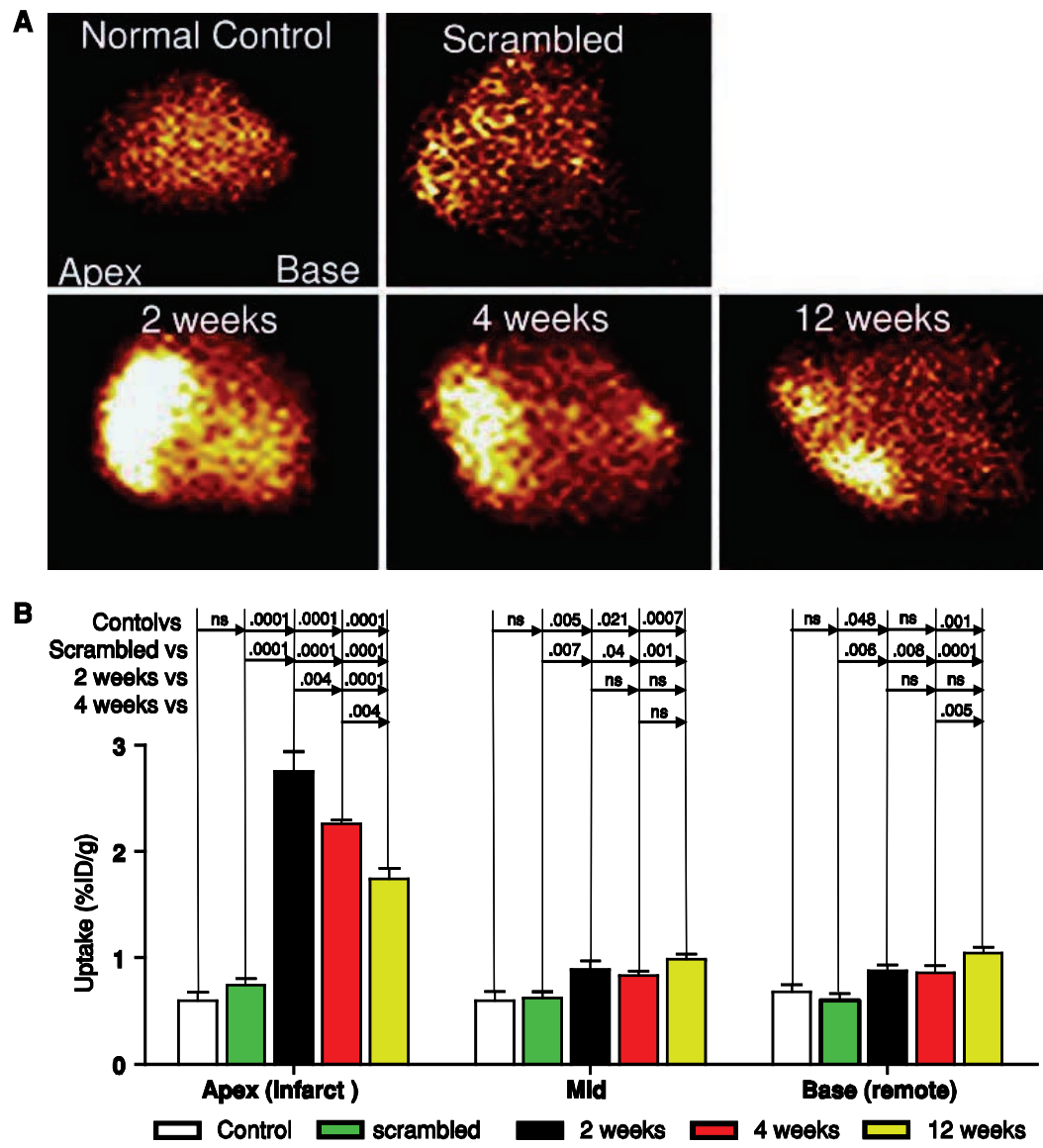

Figure 2. A, Nuclear imaging showed that no uptake was seen of the avB3 targeted tracer was seen in normal controls and in mice with infarcted hearts injected with a scrambled variant of the targeting peptide. Uptake of the tracer peaked at 2 weeks post MI, and decreased gradually at 4 weeks and 12 weeks, respectively.

$\mathbf{B}$, The in vivo imaging data are confirmed by quantitative measurement (\% injected dose per gram) in the infarct zone (left panel). In the mid sections and base sections no significant uptake of the tracer was observed

The uptake of CRIP was most pronounced in the infarcted area, and peaked at 2 weeks after MI. Immunological analysis linked CRIP uptake to myofibroblasts, and CRIP uptake 
paralleled with the production of newly formed collagen, with a yellow/green birefringence. In addition, several mice were treated with antagonists of the renin-angiotensin system, which is known to be involved in pathological myocardial remodeling. Interestingly, mice treated with captopril or with captopril and losartan displayed significantly reduced uptake of the CRIP (Figure 3). Thus, apart from the potential value of radiolabeled CRIP in visualizing collagen formation, it may also fulfill the need for a surrogate endpoint marker for therapeutic interventions.

A
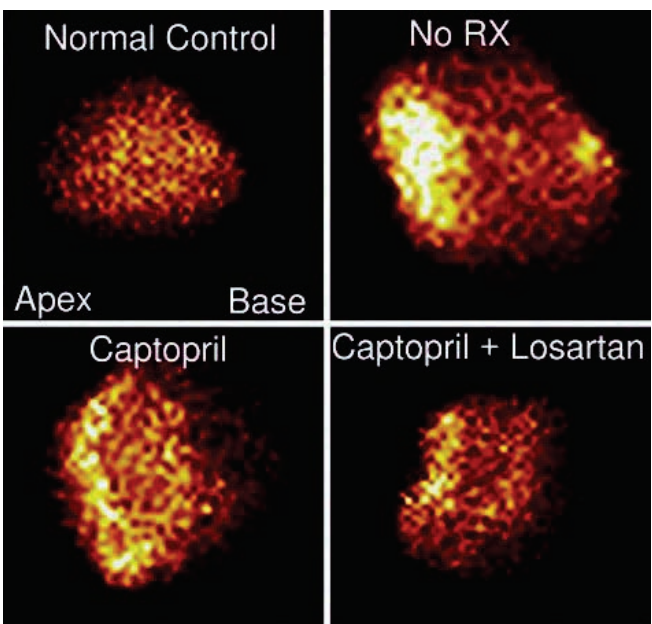

Captopril + Losartan

B
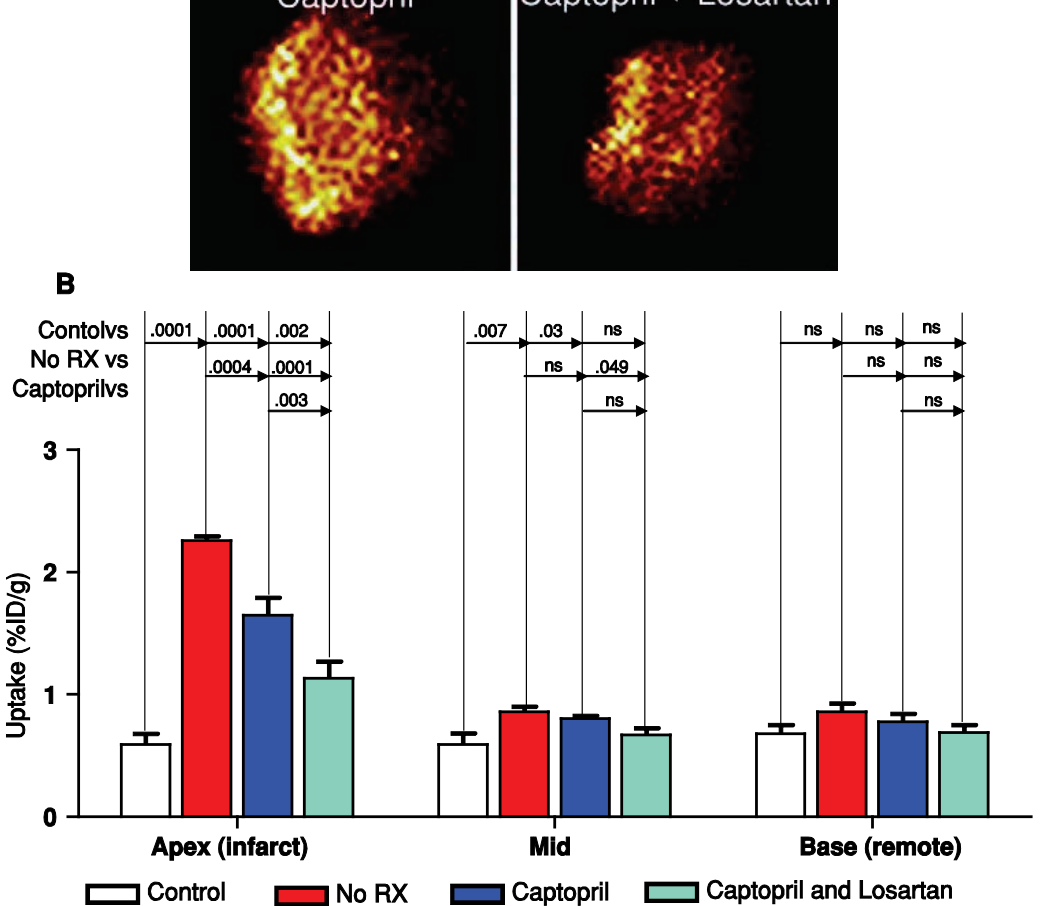

Figure 3. A, The effect of treatment with captopril and/or losartan post MI. In the treated groups, less uptake of the avB3 targeting tracer was observed using captopril and an even more extensive reduction when the treatment of captopril was combined with losartan.

$\mathrm{B}$, The imaging data were confirmed by quantitative measurement (\% injected dose per gram) 
In conclusion, imaging of avB3 integrin has the potential to identify HF-prone patients early after $\mathrm{Ml}$, and thereby will help to optimize medical treatment. However, prospective clinical trials are needed to investigate whether the potential of integrin imaging translate into clinically useful diagnostic tests.

\section{Apoptosis as a target for molecular imaging in CHF}

Apoptosis plays a key role in the process of degradation of cardiomyocytes resulting in ventricular dysfunction. ${ }^{21-24}$ Research in animal models of $\mathrm{CHF}$ has demonstrated that interference in apoptosis pathways delays the ongoing process of pump function disorders resulting in heart failure. ${ }^{25}$

One of the main biochemical characteristics of apoptosis is caspase 3 activation. The process of activation of caspase 3 , an apoptosis-related cysteine protease, begins with the release of cytochrome $\mathrm{C}$ into the cytoplasmic area, mainly caused by oxidative stress and cytokinemia. Caspases have numerous substrates, including contractile proteins, such as troponin-T. In addition, in most cells, caspase activation results in activation of DNA fragmentation enzymes. However, it is thought that the activation of DNA fragmentation enzymes in heart muscle cells is compensated for by different antiapoptotic pathways. The consequence of apoptosis activation in cardiomyocytes is that these cells become dysfunctional, but still survive due to the preservation of DNA. This means that restoration of a healthy environment could potentially restore individual cardiomyocyte function. Another consequence of caspase 3 activation is that it results in alterations in phospholipid distribution in the sarcolemmal lipid bilayer, causing revelation of phosphatidyl serine (PS) to the surface of the cell membrane. ${ }^{26,27}$ Theoretically, the extent of PS externalization reveals an indication of the degree of apoptosis in the heart. Accordingly, it is plausible that PS can be a used as a target to detect activated cell death in heart failure. The detection of PS exposure has been proven extensively by radionuclide imaging using ${ }^{99 m}$ Tc-labeled annexin-A5. ${ }^{28,29}$

Kietselaer et al recently demonstrated the feasibility of annexin-A5 imaging to reveal PCD in a small group of patients with recently diagnosed advanced heart failure. Imaging of ${ }^{99 \mathrm{~m}}$ Tc-labeled annexin-A5 was performed using a SPECT in nine CHF patients with advanced nonischemic cardiomyopathy (hypertrophic, $N=1$, dilated, $N=8$ ). To include a similar genetic background as the hypertrophic cardiomyopathy patient, the same imaging procedure was performed on two relatives who did not suffer from CHF. Left ventricular uptake of annexin-A5 was demonstrated focal, multifocal, or diffuse in a total of five patients. ${ }^{30}$ All patients in whom ${ }^{99 m}$ Tc-labeled annexin-A5 uptake was present in the left ventricle were diagnosed with a significant decrease of left ventricular function as demonstrated by follow-up echocardiography 1 year later (Figure 4). 


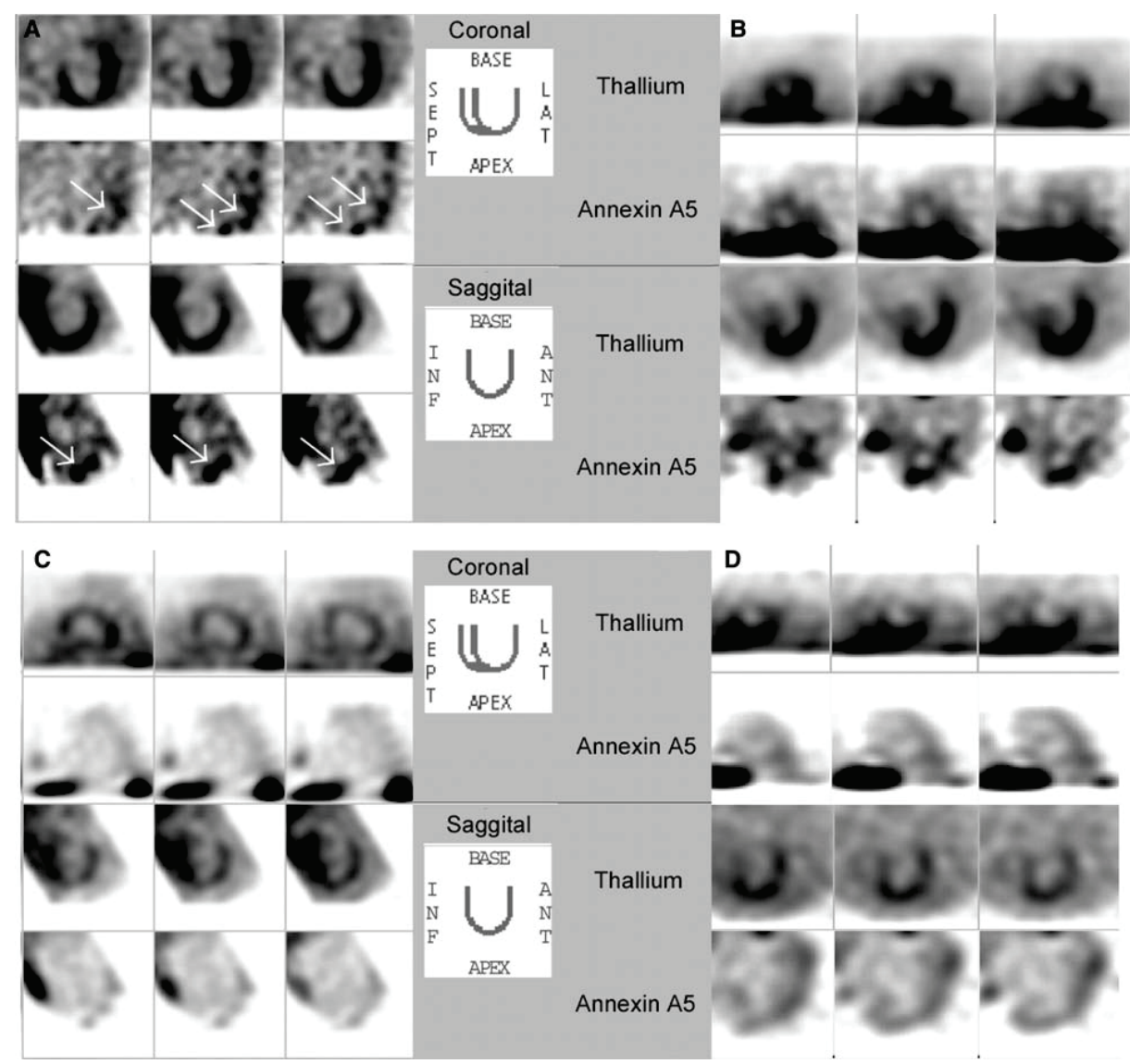

Figure 4. Uptake of ${ }^{99 m}$ Tc-labeled annexin-A5 in advanced cardiomyopathy. A, Patient with dilated cardiomyopathy (DCMP) and reduced left ventricular function showing focal uptake in the anterolateral region of the heart. B, Patient with DCMP in acute heart failure showing global uptake. C, DCMP in a patient with no symptoms of heart failure, notice no uptake is present. In D, relative of DCMP patient, absence of uptake of ${ }^{99 \mathrm{~m}} \mathrm{Tc}$ labeled annexin-A5 (adapted from Kietselaer et $\mathrm{al}^{30}$ )

The five patients who demonstrated no uptake of annexin-A5 remained in a stable clinical state or even showed improvement of left ventricular function (Figure 5). In line with the data in animal models of CHF, these data suggest that annexin-A5 uptake is related to dysfunction of the left ventricle. Annexin-A5 may provide the potential to become a useful imaging tool to identify patients with active apoptosis in the myocardium, which most likely indicates a transition to overt heart failure. The question remains what annexin-A5 binding into the myocardium indicates, since it was suggested that cardiomyocytes activate caspases during apoptosis, but that DNAses are not activated, resulting in the concept of apoptosis interruptus. 


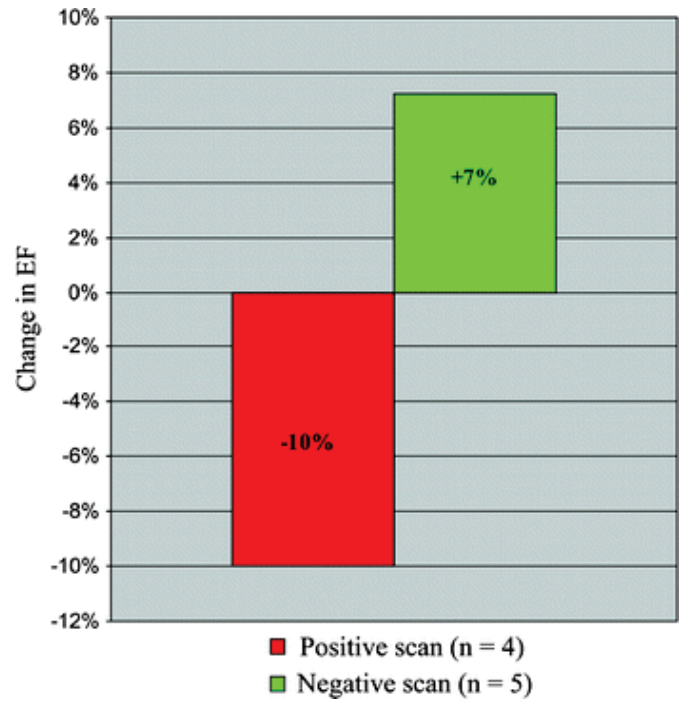

Figure 5. Differences in left ventricular ejection fraction after 1 year follow-up in CDMP patients with uptake of annexin-A5 and no uptake of annexin-A5 on SPECT (adapted from Kietselaer et $\mathrm{al}^{30}$

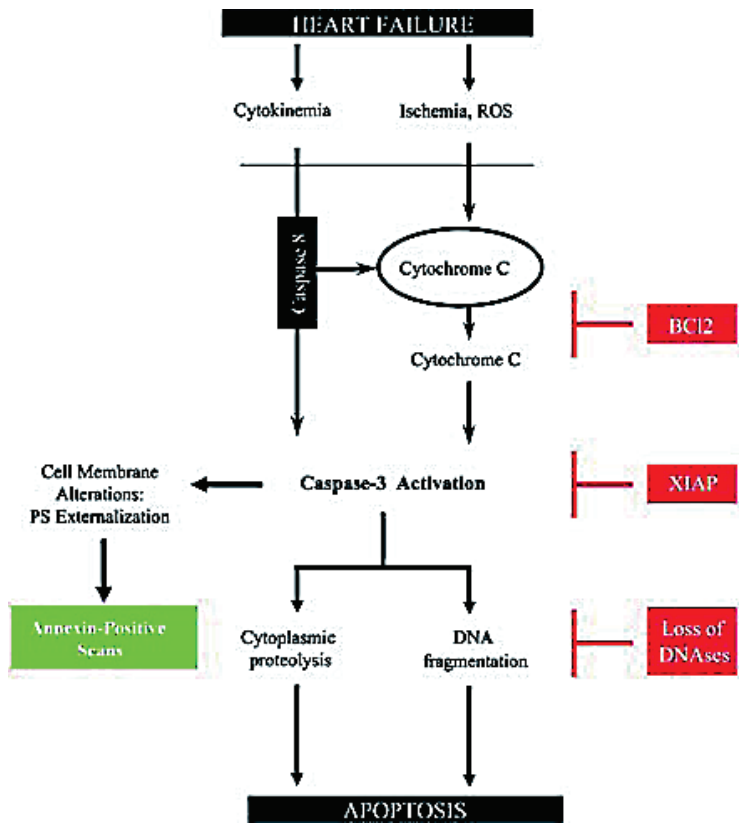

Figure 6. The concept of PS externalization in heart failure. Cytokine and oxidative stress (reactive oxygen species) inducing release of cytochrome c out of the mitochondria lead to caspase 3 activation. This results in cytoplasmic proteolysis and DNA fragmentation, ultimately leading to apoptosis. The increase of BC12- and XIAP proteins and the loss of DNAses prevent the so-called apoptosis interruptus and activate caspase 3 
Figure 6 shows a possible explanation for uptake of ${ }^{99 m}$ Tc-labeled annexin-A5 after apoptosis is stimulated. The revelation of PS is a response of caspase 3 activation. As mentioned above, different antiapoptotic pathways, preventing the degradation of DNA, compensate for the activation of DNA fragmentation enzymes in heart muscle cells. It is assumed that the quantity of PS, which manifests externally, is an indication of the amount of activated of caspase 3. Therefore, it is believed that annexin-A5 uptake not necessarily indicates loss of cardiomyocytes, but is a reflection of the activation level of caspase-3.

\section{The AT-1 receptor as a target for molecular imaging in CHF}

The essential role of the renin-angiotensin system in ventricular remodeling following AMI has been established in many experimental and clinical studies. Rather than circulating renin-angiotensin levels, it believed that myocardial upregulation of angiotensin converting enzyme, angiotensin $\mathrm{I}$, and its receptors determine the likelihood of ventricular remodeling. ${ }^{31,32}$ In an experimental study in mice, it was shown that transgenic mice with deficient angiotensin II type 1 receptor expression revealed negligible remodeling post MI. Moreover, a lower expression of fibrosis and transforming growth factor (TGFB1) was demonstrated. ${ }^{31}$ These insights from experimental models have been translated to clinical studies showing that patients with CHF patients using angiotensin receptor blockers ${ }^{33,34}$ and/or ACE-inhibitors ${ }^{35-37}$ have substantially improved survival. It has been suggested that maximization of antiangiotensin therapy, including increase in ACEinhibitor dose or addition of ARB over ACE-inhibitor therapy, could further reduce morbidity $^{33,38}$ and mortality ${ }^{39}$ in HF. It is, therefore, imaginable that accurate assessment of myocardial angiotensin receptor expression could potentially guide optimization of antiangiotensin therapy. Nowadays, diagnostic imaging of HF is focused on geometric and structural cardiac imaging. ${ }^{40,41}$

As mentioned above, it is well known that inhibition of the renin-angiotensin system, through either ACE inhibitors and/or angiotensin receptor 1 blockers, preserve cardiac function and decrease mortality post MI. ${ }^{2}$ Verjans et al investigated the potential of angiotensin receptor II type 1 imaging in an animal model of post MI left ventricular remodeling. ${ }^{42}$ A fluorescent label was marked to an angiotensin peptide analogue (APA), which traced angiotensin type 1 and 2 receptors for imaging purposes. The data from the study showed distinct uptake of the fluorescent tracer in the infarct and border zone of the mouse hearts between 1 and 6 weeks post MI (Figure 7). 

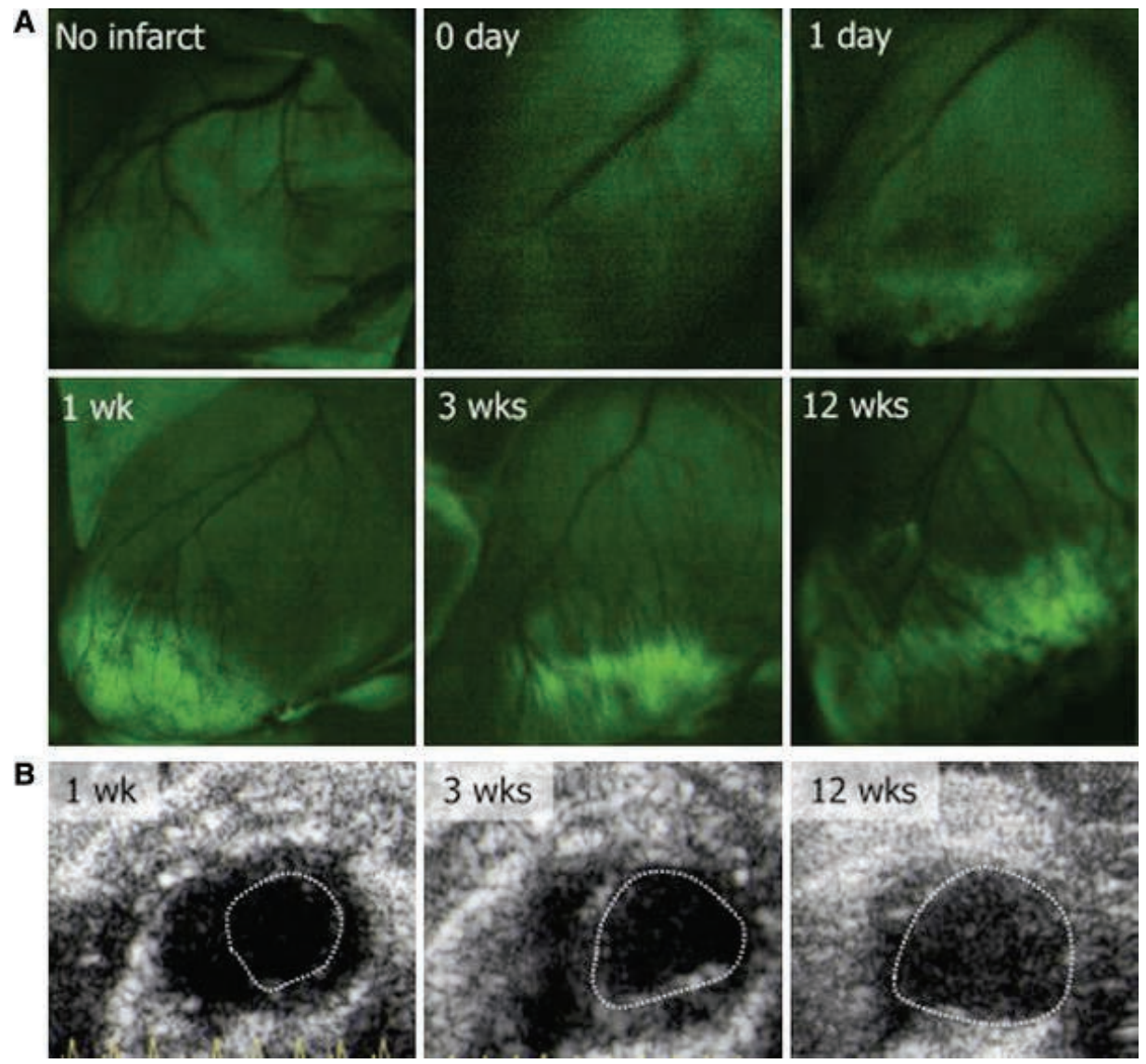

Figure 7. A, Imaging data show the uptake of the fluorescent AT1 receptor-targeted tracer at different time points after $\mathrm{MI}$. The uptake peaks at 1 week post $\mathrm{AMI}$, and then gradually decreases over time. In $\mathrm{B}$, the concomitant echocardiographic data are shown, indicating a clear increase in LV end diastolic diameter

At time point of 12 weeks, the uptake was markedly reduced. Immunohistochemical analysis and 2-photon microscopy showed co-localization of the tracer with both myofibroblasts and collagen. No uptake of the fluorescent tracer was observed in cardiomyocytes. Upregulation of the AT1 receptor on myofibroblasts allows for growth factor (such as angiotensin-II)-induced proliferation and collagen production, which is believed to contribute to healing and the remodeling process following $\mathrm{MI}^{43,44}$

The same research group created a radiolabeled imaging tracer of a well-known angiotensin II antagonist named Losartan. With the use of this analogue, SPECT-CT imaging was performed after left ventricular function was evaluated by echocardiography. These imaging studies showed that uptake of the radiolabeled Losartan product colocalized to the infarct area on SPECT-CT imaging, and that the uptake of the radiolabeled product in the infarct area was 2.4-fold higher as compared to control hearts 
(Figure 8). Together, these data demonstrate the feasibility of in vivo imaging through targeting of angiotensin receptors in an experimental HF model.
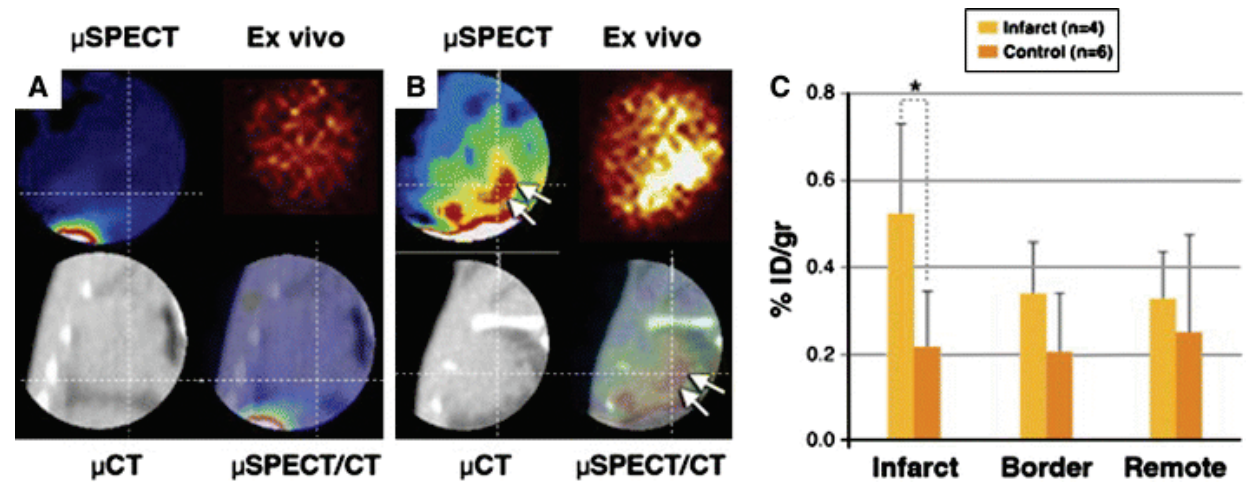

Figure 8. In A, the control mouse does not show uptake of the technetium-labeled losartan product on SPECTCT imaging. In B, in a mouse 3 week post MI clear uptake of the tracer can be seen on SPECT-CT imaging. In C, quantitative analysis (\% injected dose per gram) shows a 2.4-fold uptake of the tracer in the infarct area. In the border zone and remote zone no significant differences were seen

Furthermore, the data showed that upregulation of angiotensin receptor preceded the development of left ventricular remodeling, as detected by echocardiography. Currently, significant emphasis is being placed on the recognition of stage $A$ and $B \mathrm{HF}$ patients as a strategy of prevention of more advanced HF. ${ }^{45}$ Accordingly, development of a technology that predicts occurrence of cardiac remodeling, such as AT-1 receptor imaging, is of crucial importance, especially since the clinical practice nowadays allows diagnosis of $\mathrm{HF}$ only after the left ventricle has undergone adverse remodeling.

This concept is further emphasized by the recent demonstration of another strategy for imaging renin-angiotensin axis with the use of radiolabeled benzoyl lisinopril. Dilsizian et al incubated short-axis myocardial slices explanted from patients undergoing cardiac transplantation for end-stage ischemic cardiomyopathy with F-18 fluoro-benzoyl lisinopril. ${ }^{46}$ There was specific binding of radiotracer to ACE; mean binding was $6.6 \pm 5.2$ compared with $3.4 \pm 2.5$ luminescence $/ \mathrm{mm}^{2}$ in segments pre-incubated with cold lisinopril $(P<0.0001)$. Furthermore, mean radiotracer binding was $6.3 \pm 4.5$ in infarcted, $7.6 \pm 4.7$ in peri-infarcted, and $5.0 \pm 1.0$ luminescence $/ \mathrm{mm}^{2}$ remote noninfarcted $(P<$ 0.02 ) segments. Together, these imaging studies demonstrate that activation of the renin-angiotensin system can be visualized using molecular imaging technology. Both studies also showed that the components of the tissue renin-angiotensin cascade are upregulated only about 2 - to 3 -fold. It remains unclear whether such relatively small difference between remodeling cardiac tissue and control hearts could provide a clinically robust diagnostic strategy for imaging targeted to ATR and/or ACE. 


\section{Outlook}

Based on the preclinical and clinical data obtained in molecular imaging of adverse left ventricular remodeling, the current outlook for the development of such an imaging technology is promising. For both the imaging of avB3 integrin and imaging of phosphatidyl serine expression as a reflection of caspase 3 activation, the preliminary data provide a sufficient basis for the design and execution of novel studies. For imaging of the components of the renin-angiotensin system, the extent of uptake may be insufficient to form a basis for clinical applications. However, before one of these technologies could be adopted as diagnostic tests for routine clinical use, large clinical studies need to be conducted to address key questions. For instance, it is still unknown whether the uptake of avB3 targeting tracers is robust enough to uncover patients that are at the brink of developing adverse remodeling post MI. In addition, it remains to be seen whether the extent of the uptake of the tracer can be modulated by treatment with different regimes of CHF treating compounds. Studies focused on addressing these questions are under way.

For imaging phosphatidyl serine exposure in the heart using annexin-A5, as a reflection of caspase-3 activation, the outlook depends largely on the availability of clinically graded annexin-A5 imaging diagnostic kits. With the availability of clinical annexin-A5 imaging kits, studies could be designed to further explore the predictive value of annexin-A5 in patients with failing hearts and to study the effect of therapeutic interventions. 


\section{References}

1. Bonow RO, Smaha LA, Smith SC Jr, Mensah GA, Lenfant C. World Heart Day 2002: The international burden of cardiovascular disease: Responding to the emerging global epidemic. Circulation 2002;106:1602-5.PubMedCrossRef

2. Prabhu M, Palaian S, Malhotra A, Ravishankar P, Bista D, Almeida R, et al. Therapeutic dimensions of ACE inhibitors-A review of literature and clinical trials. Kathmandu Univ Med J (KUMJ) 2005;3:296-304.

3. Ezekowitz JA, McAlister FA. Aldosterone blockade and left ventricular dysfunction: A systematic review of randomized clinical trials. Eur Heart J 2009;30:469-77.PubMedCrossRef

4. Pitt B, Remme W, Zannad F, Neaton J, Martinez F, Roniker B, et al. Eplerenone, a selective aldosterone blocker, in patients with left ventricular dysfunction after myocardial infarction. N Engl J Med 2003;348:1309-21.PubMedCrossRef

5. van Kimmenade RR, Januzzi JL Jr, Baggish AL, Lainchbury JG, Bayes-Genis A, Richards AM, et al. Aminoterminal pro-brain natriuretic peptide, renal function, and outcomes in acute heart failure: Redefining the cardiorenal interaction? J Am Coll Cardiol 2006;48:1621-7.PubMedCrossRef

6. Sanz J, Fayad ZA. Imaging of atherosclerotic cardiovascular disease. Nature 2008;451:9537.PubMedCrossRef

7. Jaffer FA, Libby P, Weissleder R. Molecular imaging of cardiovascular disease. Circulation 2007;116:105261.PubMedCrossRef

8. Jaffer FA, Libby P, Weissleder R. Molecular and cellular imaging of atherosclerosis: Emerging applications. J Am Coll Cardiol 2006;47:1328-38.PubMedCrossRef

9. Chandrashekhar Y, Narula J. Exposing ACE up the sleeve. J Nucl Med 2007;48:173-4.PubMed

10. Frangogiannis NG. The mechanistic basis of infarct healing. Antioxid Redox Signal 2006;8:190739.PubMedCrossRef

11. Brooks PC, Clark RA, Cheresh DA. Requirement of vascular integrin alpha $v$ beta 3 for angiogenesis. Science 1994;264:569-71.PubMedCrossRef

12. Haubner R, Wester HJ, Reuning U, Senekowitsch-Schmidtke R, Diefenbach B, Kessler H, et al. Radiolabeled alpha(v)beta3 integrin antagonists: A new class of tracers for tumor targeting. J Nucl Med 1999;40:1061-71.PubMed

13. Sipkins DA, Cheresh DA, Kazemi MR, Nevin LM, Bednarski MD, Li KC. Detection of tumor angiogenesis in vivo by alphaVbeta3-targeted magnetic resonance imaging. Nat Med 1998;4:623-6.PubMedCrossRef

14. Meoli DF, Sadeghi MM, Krassilnikova S, Bourke BN, Giordano FJ, Dione DP, et al. Noninvasive imaging of myocardial angiogenesis following experimental myocardial infarction. J Clin Invest 2004;113:168491.PubMed

15. Haubner R, Kuhnast B, Mang C, Weber WA, Kessler H, Wester HJ, et al. [18F]Galacto-RGD: Synthesis, radiolabeling, metabolic stability, and radiation dose estimates. Bioconjug Chem 2004;15:619.PubMedCrossRef

16. Higuchi T, Bengel FM, Seidl S, Watzlowik P, Kessler H, Hegenloh R, et al. Assessment of alphavbeta3 integrin expression after myocardial infarction by positron emission tomography. Cardiovasc Res 2008;78:395-403.PubMedCrossRef

17. Makowski MR, Ebersberger U, Nekolla S, Schwaiger M. In vivo molecular imaging of angiogenesis, targeting alphavbeta3 integrin expression, in a patient after acute myocardial infarction. Eur Heart J 2008;29:2201.PubMedCrossRef

18. Asano Y, Ihn H, Yamane K, Jinnin M, Mimura Y, Tamaki K. Increased expression of integrin alpha(v)beta3 contributes to the establishment of autocrine TGF-beta signaling in scleroderma fibroblasts. J Immunol 2005;175:7708-18.PubMed

19. Scaffidi AK, Petrovic N, Moodley YP, Fogel-Petrovic M, Kroeger KM, Seeber RM, et al. alpha(v)beta(3) Integrin interacts with the transforming growth factor beta (TGFbeta) type II receptor to potentiate the proliferative effects of TGFbeta1 in living human lung fibroblasts. J Biol Chem 2004;279:3772633.PubMedCrossRef 
20. van den Borne SW, Isobe S, Verjans JW, Petrov A, Lovhaug D, Li P, et al. Molecular imaging of interstitial alterations in remodeling myocardium after myocardial infarction. J Am Coll Cardiol 2008;52:201728.PubMedCrossRef

21. Mani K, Kitsis RN. Myocyte apoptosis: Programming ventricular remodeling. J Am Coll Cardiol 2003;41:761-4.PubMedCrossRef

22. Hirota H, Chen J, Betz UA, Rajewsky K, Gu Y, Ross J Jr, et al. Loss of a gp130 cardiac muscle cell survival pathway is a critical event in the onset of heart failure during biomechanical stress. Cell 1999;97:18998.PubMedCrossRef

23. Olivetti G, Abbi R, Quaini F, Kajstura J, Cheng W, Nitahara JA, et al. Apoptosis in the failing human heart. N Engl J Med 1997;336:1131-41.PubMedCrossRef

24. Communal C, Sumandea M, de Tombe P, Narula J, Solaro RJ, Hajjar RJ. Functional consequences of caspase activation in cardiac myocytes. Proc Natl Acad Sci USA 2002;99:6252-6.PubMedCrossRef

25. Webster KA, Bishopric NH. Apoptosis inhibitors for heart disease. Circulation 2003;108:29546.PubMedCrossRef

26. Fadok VA, Savill JS, Haslett C, Bratton DL, Doherty DE, Campbell PA, et al. Different populations of macrophages use either the vitronectin receptor or the phosphatidylserine receptor to recognize and remove apoptotic cells. J Immunol 1992;149:4029-35.PubMed

27. Martin SJ, Reutelingsperger CP, McGahon AJ, Rader JA, van Schie RC, LaFace DM, et al. Early redistribution of plasma membrane phosphatidylserine is a general feature of apoptosis regardless of the initiating stimulus: Inhibition by overexpression of Bcl-2 and Abl. J Exp Med 1995;182:1545-56.PubMedCrossRef

28. Hofstra L, Dumont EA, Thimister PW, Heidendal GA, DeBruine AP, Elenbaas TW, et al. In vivo detection of apoptosis in an intracardiac tumor. JAMA 2001;285:1841-2.PubMedCrossRef

29. Reutelingsperger CP, Dumont E, Thimister PW, van Genderen H, Kenis H, van de Eijnde S, et al. Visualization of cell death in vivo with the annexin A5 imaging protocol. J Immunol Methods 2002;265:12332.PubMedCrossRef

30. Kietselaer BL, Reutelingsperger CP, Boersma HH, Heidendal GA, Liem IH, Crijns HJ, et al. Noninvasive detection of programmed cell loss with 99mTc-labeled annexin A5 in heart failure. J Nucl Med 2007;48:562-7.PubMedCrossRef

31. Harada K, Sugaya T, Murakami K, Yazaki Y, Komuro I. Angiotensin II type 1A receptor knockout mice display less left ventricular remodeling and improved survival after myocardial infarction. Circulation 1999;100:2093-9.PubMed

32. Lamas GA, Pfeffer MA. Left ventricular remodeling after acute myocardial infarction: Clinical course and beneficial effects of angiotensin-converting enzyme inhibition. Am Heart J 1991;121:1194202.PubMedCrossRef

33. Cohn JN, Tognoni G. A randomized trial of the angiotensin-receptor blocker valsartan in chronic heart failure. N Engl J Med 2001;345:1667-75.PubMedCrossRef

34. Dickstein K, Kjekshus J. Effects of losartan and captopril on mortality and morbidity in high-risk patients after acute myocardial infarction: The OPTIMAAL randomised trial. Optimal Trial in Myocardial Infarction with Angiotensin II Antagonist Losartan. Lancet 2002;360:752-60.PubMedCrossRef

35. Effect of ramipril on mortality and morbidity of survivors of acute myocardial infarction with clinical evidence of heart failure. The Acute Infarction Ramipril Efficacy (AIRE) Study Investigators. Lancet 1993;342:821-828.

36. Pfeffer MA, Braunwald E, Moye LA, Basta L, Brown EJ Jr, Cuddy TE, et al. Effect of captopril on mortality and morbidity in patients with left ventricular dysfunction after myocardial infarction. Results of the survival and ventricular enlargement trial. The SAVE Investigators. N Engl J Med 1992;327:669-77.PubMed

37. Kober L, Torp-Pedersen C, Carlsen JE, Bagger H, Eliasen P, Lyngborg K, et al. A clinical trial of the angiotensin-converting-enzyme inhibitor trandolapril in patients with left ventricular dysfunction after myocardial infarction. Trandolapril Cardiac Evaluation (TRACE) Study Group. N Engl J Med 1995;333:16706.PubMedCrossRef 
38. Packer M, Poole-Wilson PA, Armstrong PW, Cleland JG, Horowitz JD, Massie BM, et al. Comparative effects of low and high doses of the angiotensin-converting enzyme inhibitor, lisinopril, on morbidity and mortality in chronic heart failure. ATLAS Study Group. Circulation 1999;100:2312-8.PubMed

39. Pfeffer MA, Swedberg K, Granger CB, Held P, McMurray JJ, Michelson EL, et al. Effects of candesartan on mortality and morbidity in patients with chronic heart failure: the CHARM-Overall programme. Lancet 2003;362:759-66.PubMedCrossRef

40. Narula J, Acio ER, Narula N, Samuels LE, Fyfe B, Wood D, et al. Annexin-V imaging for noninvasive detection of cardiac allograft rejection. Nat Med 2001;7:1347-52.PubMedCrossRef

41. Narula J, Khaw BA, Dec GW Jr, Palacios IF, Southern JF, Fallon JT, et al. Brief report: recognition of acute myocarditis masquerading as acute myocardial infarction. N Engl J Med 1993;328:1004.PubMedCrossRef

42. Verjans JWH, Lovhaug D, Narula N, Petrov AD, Indrevoll B, Bjurgert E, et al. Noninvasive imaging of angiotensin receptors after myocardial infarction. JACC Cardiovasc Imaging 2008;1:354-62. PubMedCrossRef

43. Weber KT. Extracellular matrix remodeling in heart failure: A role for de novo angiotensin II generation. Circulation 1997;96:4065-82.PubMed

44. Weber KT, Brilla CG. Pathological hypertrophy and cardiac interstitium. Fibrosis and renin-angiotensinaldosterone system. Circulation 1991;83:1849-65.PubMed

45. Hunt SA. ACC/AHA 2005 guideline update for the diagnosis and management of chronic heart failure in the adult: A report of the American College of Cardiology/American Heart Association Task Force on Practice Guidelines (Writing Committee to Update the 2001 Guidelines for the Evaluation and Management of Heart Failure). J Am Coll Cardiol 2005;46:e1-82.PubMedCrossRef

46. Dilsizian V, Eckelman WC, Loredo ML, Jagoda EM, Shirani J. Evidence for tissue angiotensin-converting enzyme in explanted hearts of ischemic cardiomyopathy using targeted radiotracer technique. J Nucl Med 2007;48:182-7.PubMed 



\section{CHAPTER 3}

\section{Macrophage depletion in hypertensive}

rats accelerates development of

cardiomyopathy

H.R. Zandbergen*, U.C. Sharma, S. Gupta*, J.W.H. Verjans, S.W.M. van den Borne, S., T. van Brakel, A. Duijvestijn, N. van Rooijen, J.G. Maessen, C. Reutelingsperger, Y. M. Pinto, J. Narula, and Leo Hofstra

*contributed equally 


\section{Abstract}

Inflammation contributes to the process of ventricular remodeling after acute myocardial injury. To investigate the role of macrophages in the chronic process of cardiac remodeling, they were selectively depleted by intravenous administration of liposomal clodronate in heart failure-prone hypertensive Ren-2 rats from the age of 7 until 13 weeks. Plain liposomes were used for comparison. Liposomal clodronate treatment reduced the number of blood monocytes and decreased the number of macrophages in the myocardium. Compared to plain liposomes, liposomal clodronate treatment rapidly worsened left ventricular ejection function in hypertensive rats. Liposomal clodronatetreated Ren-2 rat hearts showed areas of myocyte loss with abundant inflammatory cell infiltration, predominantly comprising CD4 positive T lymphocytes. The current study showed that lack of macrophages was associated with earlier development of myocardial dysfunction in hypertensive rats. Modulation of macrophage function may be of value in the evolution of cardiomyopathy.

Keywords: hypertension; inflammation; ventricular remodeling; heart failure 


\section{Introduction}

Although inflammatory myocardial diseases are well known to induce chronic cardiomyopathic state, sparse inflammatory cell infiltration is commonly observed in noninflammatory heart muscle disease. ${ }^{1,2}$ It is believed that inflammatory mechanisms contribute to chronic cardiomyopathy by slow and progressive tissue damage and alteration of myocardial architecture or induction of autoimmunity. ${ }^{3,4}$

Macrophages may also contribute to myocardial dysfunction by producing inflammatory and profibrotic mediators. ${ }^{5}$ Accumulating evidence on the patho- physiological consequences of sustained expression of macrophage-based proinflammatory mediators in preclinical and clinical heart failure (HF) models has led to several clinical trials that have used targeted approaches to neutralize tumor necrosis factor (TNF) in patients with moderate to advanced HF. ${ }^{6,7}$ However, these targeted approaches have not yielded the anticipated results. In the current study, we investigated the role of macrophages in evolution of ventricular dysfunction in hypertensive homozygous Ren-2 rats. These animals are known to demonstrate rapid progression from hypertension to compensated left ventricular hypertrophy and HF. Male homozygous transgenic rats carrying 2 copies of mouse renin-2 genes (Ren-2 rats) exhibit moderate hypertension, myocardial hypertrophy, and excessive fibrosis associated with overproduction of angiotensin-II (Ang-II). These pathological changes can begin as early as 6 weeks of age and $50 \%$ of these rats progress to HF by 12 to 14 weeks, while the other half remain compensated at least till 17 weeks. The failing Ren-2 rat hearts reveal abundant macrophage infiltration. We hypothesized that antagonizing macrophage influence in the heart by a depletion strategy would result in a delay in the development of end- stage hypertensive cardiomyopathy and HF. For this purpose, we injected liposomal clodronate (LC) intravenously in homozygous Ren-2 rats, for selective elimination of macrophages. ${ }^{8,9}$ Liposomal clodronate is phagocytosed by circulating monocytes. Free clodronate does not cross cell membranes and has an extremely short half-life in the circulation. Liposomal clodronate is removed from the circulation by the renal system and has no major adverse effects on cell types that do not phagocytose the liposomes. ${ }^{8-10}$

\section{Methods}

\section{Experimental Design}

Male, 6-week-old homozygous Ren-2 and Sprague Dawley (SD) rats were obtained from M\&B (Bom- holtvej, Denmark). Clodronate was a gift from Roche Diagnostics $\mathrm{GmbH}$, Mannheim, Germany. Liposomal clodronate and plain liposomes (PL) were prepared as described previously. ${ }^{9}$ Under light isoflurane anesthesia, 10 male homozygous Ren-2 rats received $\mathrm{LC}$ ( $4 \mathrm{~mL} / \mathrm{gram}$ body weight) through the tail vein, every week for 7 weeks. As a 
control for the liposomally encapsulated clodronate, liposomes filled with phosphate buffer saline (PBS) were injected in 7 Ren-2 rats. In addition, 4 healthy SD rats received LC and 4 age-matched SD rats received PL. Rats were euthanized at 13 weeks. In all experimental groups, blood pressure was measured by tailcuff method, and echocardiography was performed at $0,14,28$, and 42 days of $L C$ or PL treatment in rats sedated with $2 \%$ isoflurane. Standard views were obtained in 2-dimensional as well as M-mode with a 12-MHz transducer (Sonos 5500, Hewlett Packard, Netherlands) with approximately 220 frames per recording and the data analysis was performed by a blinded observer. The Institutional Animal Care and Use Committee approved the procedure for care and treatment of animals.

\section{Peripheral Blood Monocytes and CD4p and CD8p T cells}

Peripheral blood monocytes and CD4p and CD8p T cells were analyzed in Ren-2 rats at 2 and 7 days after intravenous infusion of LC or PL with specific antibodies by flow cytometry. In brief, anticoagulated blood $(200 \mathrm{~mL})$ was incubated for 30 minutes $(40 \mathrm{C})$ with mouse anti-rat R-phycoerythrin (RPE)-conjugated anti-CD4, CD8, and CD68 antibodies (Serotec, Dus- seldorf, Germany) and isotype controls. All analyses were performed separately. Fluorescence-activated cell sorting (FACS) lysing solution (1:20 dilution) was added for 15 minutes. The residual cells were washed ( $x 1500 \mathrm{rpm}, 5$ minutes, 4o C) in FACS medium (PBS, 1\% bovine serum albumin [BSA], 0.02\% sodium azide) and suspended for flow cytometry. Monocytes and lymphocytes were identified according to their relative size, side scattering, and fluorescence.

\section{Immunohistochemical localization of macrophages and T lymphocytes in myocardium}

Immunohistochemistry was performed on cryofixed myocardial sections. After drying the sections for 15 minutes, ice-cold methanol (100\%) was added for 10 minutes; sections were washed 3 times with PBS and blocked with 5\% horse serum for 60 minutes. Anti-CD3, -CD4, -CD8, and -CD68 (Serotec) and anti-annexin A5 (BioVision, Uithoorn, The Netherlands) antibodies (1:250 in 0.1\% BSA in PBS) were applied and incubated overnight at $4^{\circ} \mathrm{C}$. Horseradish peroxidase-conjugated secondary antibodies were then used for 4 hours at room temperature followed by horseradish peroxidase-labeled streptavidin for 60 minutes. After washing 3 times for 5 minutes in PBS, 3,30-diaminobenzidine tetrahydrochloride reagent was applied to visualize the reaction. 


\section{Statistical Analyses}

Data are presented as mean + SD. The comparisons were made by unpaired t test. For multiple comparisons, 1-way analysis of variance (ANOVA) in combination with a Dunnett's post hoc analysis was made. Analyses were performed by the use of the statistical package SPSS 10.0. Probability value of $P<.05$ was considered to be statistically significant.

\section{Results}

\section{Peripheral Blood Monocyte Count and the Interstitial Macrophage Infiltration}

Baseline peripheral blood monocyte proportion in PL-treated Ren-2 rats was $2.28 \%+$ $0.15 \%$ (Figure $1 \mathrm{~A}$ and $\mathrm{B}$ ). Two days after LC injection in Ren-2 rats, the number of monocytes sharply reduced to $0.81 \%$

$+0.21 \%$ (Figure $1 \mathrm{C}$ and D), whereas CD4 and CD8 count remained unchanged (CD4: LC, $43.6 \%+3.5 \%$ vs. PL, $45+4.6$; CD8: LC, $5 \%+2.5 \%$ vs. PL, $4.8+1.9$, P = NS; Table 1). However, the monocyte count returned to baseline levels $(1.8+0.38)$ by 7 days. Presence of macrophages in the hearts was shown by CD68-specific monoclonal antibody. In the Ren-2 rats that received PL, macrophages were frequently observed (Table 2). However, LC-treated Ren-2 rats showed scattered cells identical to macrophage morphology; the macrophages also stained positively for annexin A5, suggesting LC- induced apoptotic changes in these cells (Figure 2A). Annexin A5 positivity was not seen in the PL-treated Ren-2 rats (Figure $2 \mathrm{~B}$ ) and control rats with $\mathrm{LC}$ (Figure $2 \mathrm{C}$ ) and $\mathrm{PL}$ (Figure 2D) treatment.

Table 1. Monocyte, CD4, and CD8 Count of LC- and PL-treated Rats (\%)a

\begin{tabular}{lll}
\hline & Ren-2-LC & Ren-2-PL \\
\hline Monocytes & $0.81+0.21$ & $2.28+0.15$ \\
T-helper cells (CD4) & $43.6+3.5$ & $45+4.6$ \\
Cytotoxic T cells (CD8) & $5+2.5$ & $4.8+1.9$ \\
\hline
\end{tabular}

Abbreviations: LC, liposomal clodronate; PBS, phosphate buffer saline; PL, PBS liposomes. ${ }^{\mathrm{a}} \mathrm{P}=\mathrm{NS}$. 
Table 2. Morphometric Data to Compare the Cellular Infiltration and Apoptotic Changes in the Myocardium of LC- or PL-treated Rats

\begin{tabular}{lllll}
\hline \multicolumn{5}{l}{ Cell counts/HPF } \\
\cline { 2 - 5 } & $\begin{array}{l}\text { Ren-2-LC } \\
(\mathrm{N}=7)\end{array}$ & $\begin{array}{l}\text { Ren-2-PL } \\
(\mathrm{N}=7)\end{array}$ & $\begin{array}{l}\text { SD-LC } \\
(\mathrm{N}=4)\end{array}$ & $\begin{array}{l}\text { SD-PL } \\
(\mathrm{N}=4)\end{array}$ \\
\hline Mononuclear cells (HE-staining) & $89.3+26 \mathrm{a}$ & $7+2.4$ & $13.8+2.1$ & $8.5+1$ \\
Macrophages (CD68) & $1.8+0.31$ & $13.8+3.3^{\mathrm{b}}$ & $0.66+0.4$ & $2.5+0.7$ \\
T-lymphocytes (CD3) & $49+13.3^{\mathrm{a}}$ & $3.3+0.68$ & $4.8+1.1$ & $4.3+0.8$ \\
T-helper cells (CD4) & $33.8+13.7^{\mathrm{a}}$ & & & $3.1+0.8$ \\
Cytotoxic T-cells (CD8) & $2.8+0.4$ & $2.3+0.50$ & $2.1+0.4$ & $2.3+0.51$ \\
Apoptotic cells (Annexin A5) & $10.5+4.2^{\mathrm{a}}$ & $2.1+0.66$ & $3.1+0.7$ & $1.1+0.4$ \\
\hline
\end{tabular}

Abbreviations: HE, hematoxylin-eosin; HPF, high power field; LC, liposomal clodronate; PBS, phosphate buffer saline; PL, PBS liposomes; SD, Sprague Dawley.

${ }^{a} \mathrm{P}<.05$ versus Ren-2-PL, SD-LC, and SD-PL; ${ }^{\mathrm{b}} \mathrm{P}<.05$ versus Ren-2-LC, SD-LC, and SD-PL.
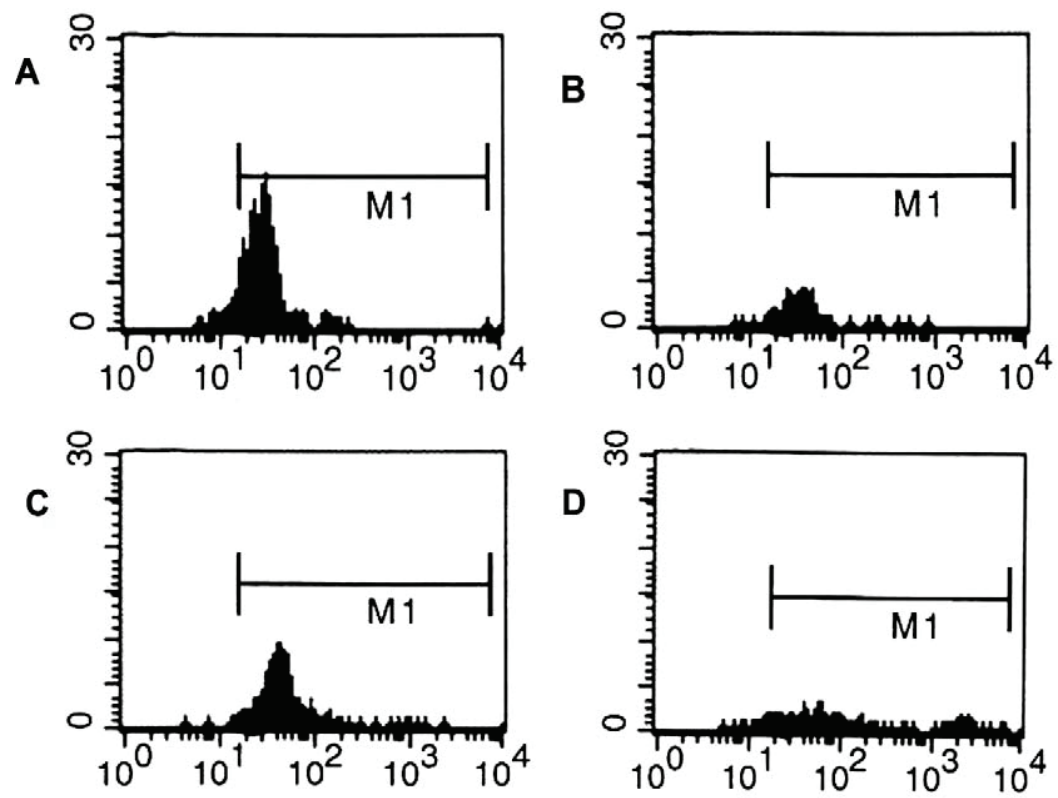

Figure 1. Flow cytometric analysis of blood monocyte population after the infusion of LC or PL in Ren-2 rats. We injected PL or LC in Ren-2 rats and collected the blood samples after 48 hours. Height of the histogram represents monocyte population. A, FACS analysis of CD68 positive cells in the blood in PL-treated Ren-2 rats. B, Isotype control for panel A. C, CD68 positive cells in LC-treated Ren-2 rats. D. Isotype control for panel C. FACS = fluorescence-activated cell sorting; $L C=$ liposomal clodronate; $P L=$ plain liposomes. 

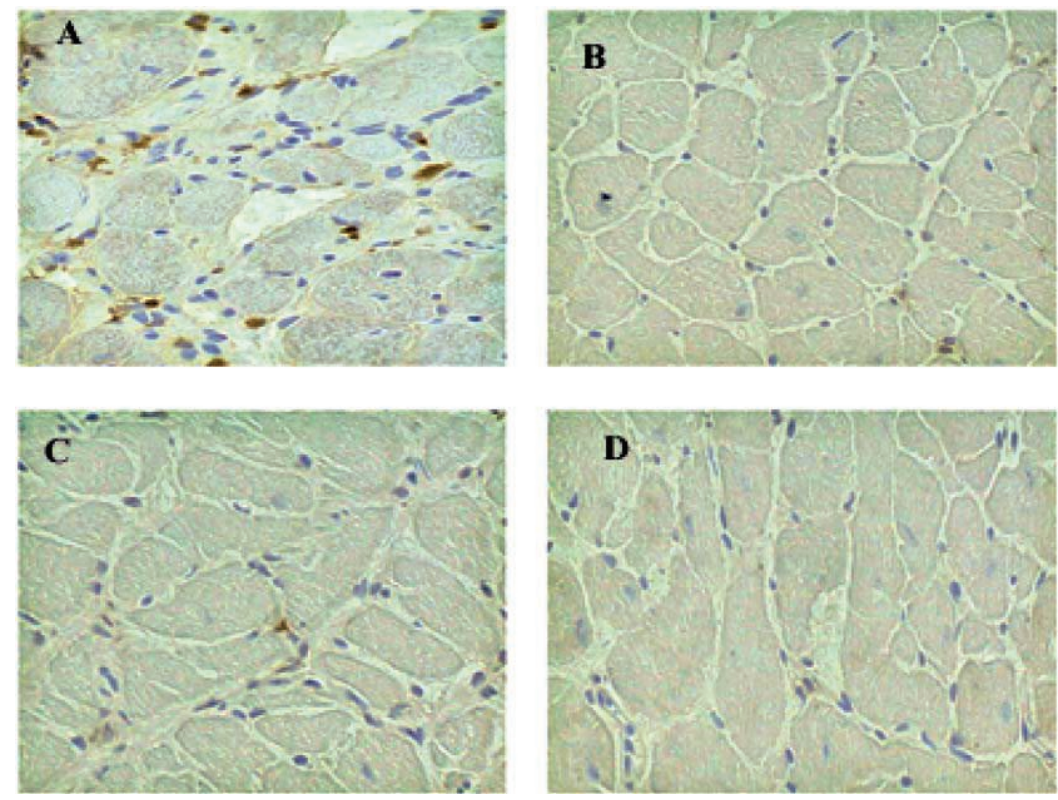

Figure 2. Demonstration of apoptosis-specific changes by annexin A5 positivity. A, Anti-annexin A5 staining showing apoptosis of macrophages in LC-treated Ren-2 rat hearts. B-D, Anti-annexin A5 staining in PL-treated Ren-2 rats, LC-treated SD rats, and PL-treated SD rats, respectively. $\mathrm{LC}=$ liposomal clodronate; PL = plain liposomes; $\mathrm{SD}=$ Sprague Dawley.

\section{Evolution of Cardiac Function in Monocyte-depleted Ren-2 Rats}

The Ren-2 rats that received LC from 7 to 13 weeks of age demonstrated significant deterioration of myocardial function (ejection fraction (EF) \%, baseline, $65.2+4.1 ; 6$ weeks, $49+5.4, \mathrm{P}<.05)$. However, Ren-2 rats that received $\mathrm{PL}$ showed only a mild and no decrease in cardiac performance (EF\%, baseline, $67+3.1 ; 6$ weeks, $62+5.3$ ). The control rats did not show any significant alteration in cardiac perfor-mance, regardless of LC (EF\%, baseline, $64+4 ; 6$ weeks, $63+3.8$ ) or PL (EF\%, baseline, $65.5+1.9 ; 6$ weeks, $66.4+1.0$ ) administration (Table 3 ). This suggests that persistent macrophage depletion in hypertensive hearts (and normally with abundant macrophage infiltration) accelerates functional dete- rioration. There was no significant difference in body weight between the 4 experimental groups. Ren-2 rats were hypertensive and had significantly increased heart weight to body weight ratio compared to controls. However, these parameters were not significantly different between LC- and PL-treated rats (Table 4).

\section{Histological Consequences of Macrophage Depletion}

The Ren- 2 rats that received LC treatment for 6 weeks showed areas of cardiomyocyte damage (Figure 3A), which was not discernible in control groups (Figure 3B-D). Although there was a marked decrease in number of macrophages, abundant focal infiltrates of 
mononuclear cells were noted in the myocardium in LC-treated Ren-2 rat hearts. Morphologically, the infiltrated cells showed round nucleus with myocardial interstitial pattern of dis- tribution (Figure 3A). Immunohistochemical analysis revealed that these round cells expressed CD3 (Figure 4) and CD4 antigen, suggesting that the macrophagedepleted Ren-2 rat hearts harbor increased CD4p T-helper cells in the myocardium (Table 2).

Table 3. Baseline, 2-,4-, and 6-Week Echocardiographic Parameters in LC- or PL-treated Ren-2 Rats and SD Controls

\begin{tabular}{llllll}
\hline $\begin{array}{l}\text { LV Functional } \\
\text { Parameters }\end{array}$ & Time Point & $\begin{array}{l}\text { Ren-2-LC } \\
(\mathrm{N}=10)\end{array}$ & $\begin{array}{l}\text { Ren-2-PL } \\
(\mathrm{N}=7)\end{array}$ & $\begin{array}{l}\text { SD-LC } \\
(\mathrm{N}=4)\end{array}$ & $\begin{array}{l}\text { SD-PL } \\
(\mathrm{N}=4)\end{array}$ \\
\hline EF (\%) & Baseline & $65.2+4.1$ & $67+3.1$ & $64+4$ & $65.5+1.9$ \\
& 2 weeks & $64.6+4.9$ & $66.9+3.04$ & $63.3+3.4$ & $63.7+2.7$ \\
& 4 weeks & $57+6.6$ & $64.3+6.4$ & $62.6+2.3$ & $64.5+3.9$ \\
& 6 weeks & $49+5.4^{\mathrm{a}}$ & $62+5.3$ & $63+3.8$ & $66.4+1.0$ \\
FS (\%) & $32.7+4.2$ & $34.8+4.2$ & $31.2+3.8$ & $36.7+0.5$ \\
& Baseline & $30+4$ & $30.2+4$ & $30.2+1.5$ & $35.2+1.2$ \\
& 2 weeks & $26+3.2$ & $26.5+3.2$ & $28.5+3.7$ & $34.2+2.2$ \\
& 6 weeks & $22.8+4.8^{\mathrm{a}}$ & $30.2+4.2$ & $30.7+3.5$ & $34.4+3.2$ \\
& Baseline & $0.41+0.26$ & $0.40+0.17$ & $0.44+0.15$ & $0.40+0.07$ \\
& 2 weeks & $0.55+0.21$ & $0.41+0.16$ & $0.41+0.1$ & $0.4+0.05$ \\
& 4 weeks & $0.81+0.35$ & $0.43+0.09$ & $0.41+0.07$ & $0.4+0.06$ \\
& 6 weeks & $1.1+0.68^{\mathrm{a}}$ & $0.43+0.16$ & $0.42+0.03$ & $0.42+0.07$ \\
\hline
\end{tabular}

Abbreviations: EF, LV ejection fraction; EDV, end diastolic volume; FS, LV fractional shortening; LC, liposomal clodronate; LV, left ventricular; PBS, phosphate buffer saline; PL, PBS liposome; SD, Sprague Dawley.

${ }^{\mathrm{a}} \mathrm{P}<.05$ versus Ren-2 placebo and SD rats.

Table 4. Blood Pressure, Heart Weight, and Body Weight of LC- and PL-treated Rats

\begin{tabular}{lllll}
\hline & $\begin{array}{l}\text { Ren-2-LC } \\
(\mathrm{N}+7)\end{array}$ & $\begin{array}{l}\text { Ren-2-PL } \\
(\mathrm{N}=7)\end{array}$ & $\begin{array}{l}\text { SD-LC } \\
(\mathrm{N}=4)\end{array}$ & $\begin{array}{l}\text { SD-PL } \\
(\mathrm{N}=4)\end{array}$ \\
\hline $\mathrm{BP}(\mathrm{mm} \mathrm{Hg})$ & $253+15^{\mathrm{a}}$ & $259+10^{\mathrm{a}}$ & $131+4.7$ & $128+10$ \\
$\mathrm{BW}(\mathrm{g})$ & $314+18$ & $310+23$ & $314+13.5$ & $308+8.9$ \\
\hline $\mathrm{HW} / \mathrm{BW}(\%)$ & $0.47+0.04^{\mathrm{a}}$ & $0.50+0.05^{\mathrm{a}}$ & $0.36+0.03$ & $0.35+0.03$ \\
\hline
\end{tabular}

Abbreviations: BP, blood pressure; BW, body weight; HW/BW, heart weight to body weight ratio; LC, liposomal clodronate; PBS, phosphate buffer saline; PL, PBS liposomes; SD, Sprague Dawley.

${ }^{a} \mathrm{P}<.05$ versus SD-LC and SD-PL. 
A

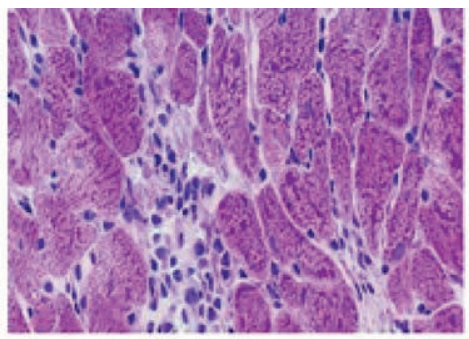

\section{C}

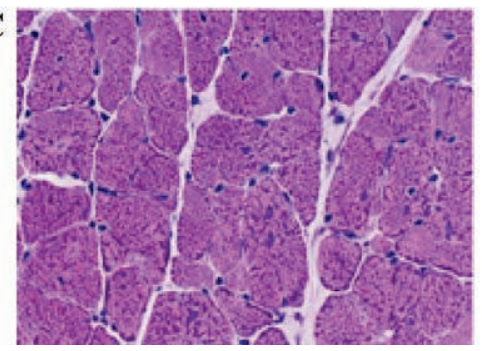

B

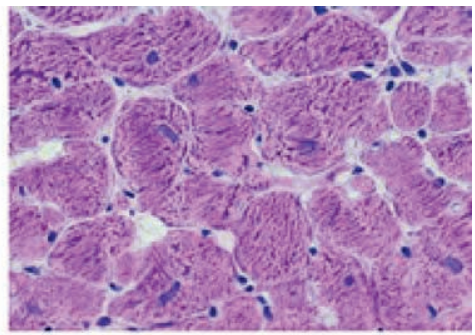

D

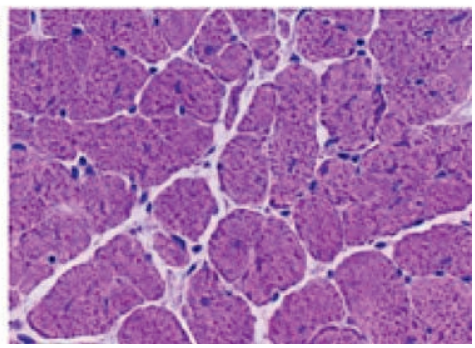

Figure 3. Histological characterization of the myocardial changes in LC-treated Ren-2 rats and controls. A, Hematoxylin-eosin stained myocardial sections showed areas of cardiomyocyte damage with mononuclear cellular infiltration in LC-treated Ren-2 rats. These changes were not remarkable in PL-treated Ren-2 (panel B), LC-treated SD (panel C), and PL-treated SD (panel D) rat hearts. $\mathrm{LC}=$ liposomal clodronate; $\mathrm{PL}=$ plain liposomes; $\mathrm{SD}=$ Sprague Dawley.
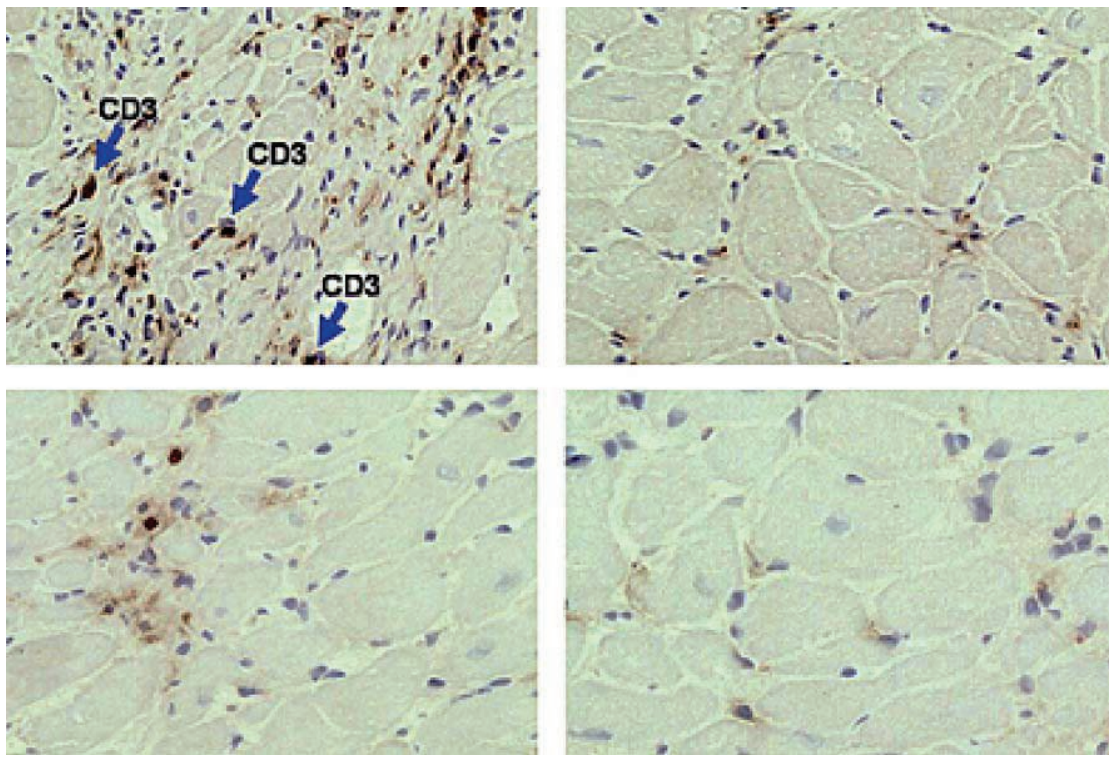

Figure 4. Demonstration of T-lymphocyte infiltration in LC-treated Ren-2 rat hearts. A, Anti-CD3 staining showing abundance of $T$ lymphocytes in the hearts of Ren-2-rats treated with LC. B-D, Anti-CD3 staining in PL-treated Ren-2, LC-treated SD, and PL-treated SD rat hearts, respectively. 


\section{Discussion}

In contrast to our beginning hypothesis, the results of the current study suggest that the depletion of macrophages, in a model of hypertensive heart disease, results in the accelerated development of end-stage hypertensive cardiomyopathy and HF. The macrophage-depleted hearts showed infiltration with CD4p T lymphocyte and multifocal loss of cardiomyocytes.

The possible role of macrophage infiltration in cardiomyopathy can be discussed as follows. First, minimal fibrosis was demonstrated and neither fibroblasts nor collagen deposition was observed. It is known that macrophage-related cytokines are associated with myofibroblast proliferation and collagen production. Although development of interstitial fibrosis is an important component of cardiomyopathic process, it is reasonable to observe that complete abolition of fibrosis should be detrimental. In a postinfarction model, macrophage depletion has been reported to be associated with acute ventricular dilatation and early onset of HF. ${ }^{11}$ It has also been demonstrated that intramyocardial injection of collagen prevents ventricular aneurysmal formation and $\mathrm{HF}^{12}$ Although these experiments highlight the importance of the collagen framework in healing from myocardial injury, neurohumoral antagonists (which have proven to be effective in prevention of remodeling and longevity) are known to reduce collagen deposition. ${ }^{13}$ It is therefore likely that both excessive collagen deposition as well as lack of collagen deposit is adversorial in myocardial remodeling. Second, cardiomyopathies are associated with increased circulating levels of various cytokines, including TNF- $\alpha$, which are known to be detrimental for myocardial function. ${ }^{14}$ However, blocking of TNF- $\alpha$ has paradoxically resulted in worsening of experimental chronic Chagas disease-mediated cardiomyopathy, ${ }^{15}$ which is similar to our observations. Third, macrophage-depleted hearts showed abundant T-lymphocyte infiltration. The mechanisms for compensatory CD4p T-cell infiltration in macrophage-depleted hypertensive rats remain unclear. At least 4 distinct subsets of CD4p T cells have been recognized including Th1, Th2, Treg, and Th17 cells. Treg appear to play a protective role ${ }^{16}$ and Th2 cells play a role in antibody-mediated response; as such CD4p T cells in our study are unlikely to be Treg or Th2 cells. Therefore, we assume that the CD4p T-cell infiltrates may be composed of either Th1 cells, Th17 cells, or both. Th17 cells secrete a number of proinflammatory cytokines, including inter-leukin (IL)-17, and have shown to play an important role in autoimmunity and inflammation. ${ }^{17-19}$ It has been shown that IL-7-mediates experimental autoimmune cardiomyopathy in t-bet knock out mice (t-bet favors Th1 development), suggesting a role of IL-17 in cardiomyopathy. ${ }^{20}$

Taken together, sustained depletion of macrophages could contribute to the deterioration of cardiac function because of the loss of the beneficial aspects of macrophage function and/or deleterious effects of enhanced CD4p T-cell infiltration. 


\section{Conclusions}

The results of the current study show a potentially important role of macrophages in ventricular remodeling in hypertension. Macrophage activation may be important in repair processes and debris clearance and hence depletion of macrophages may not necessarily be an effective strategy in prevention of HF. However, larger studies may be needed to evaluate the role of modest modulation of macrophage function.

\section{Acknowledgments}

This study was supported by a VIDI grant (016.036.346) from the Netherlands Organization for Scientific Research (NWO) to Dr Y. M. Pinto. We acknowledge the technical assistance of Ms Maria Vroomen. 


\section{References}

1. Vasan RS, Sullivan LM, Roubenoff R, et al. Framingham Heart Study. Inflammatory markers and risk of heart failure in elderly subjects without prior myocardial infarction: the Framingham Heart Study. Circulation. 2003;107:1486-1491.

2. Weber KT, Gerling IC, Kiani MF, et al. Aldosteronism in heart failure: a proinflammatory/fibrogenic cardiac phe- notype. Search for biomarkers and potential drug targets. Curr Drug Targets. 2003;4:505-516.

3. Mann DL. Inflammatory mediators and the failing heart: past, present, and the foreseeable future. Circ Res. 2002;91:988-998.

4. Shioi T, Matsumori A, Kihara Y, et al. Increased expres- sion of interleukin-1 beta and monocyte chemotactic and activating factor/monocyte chemoattractant protein-1 in the hypertrophied and failing heart with pressure overload. Circ Res. 1997;81:664-671.

5. Sharma UC, Pokharel S, van Brakel TJ, et al. Galectin-3 marks activated macrophages in failure-prone hypertro- phied hearts and contributes to cardiac dysfunction. Circulation. 2004;110:3121-3128.

6. Chung ES, Packer M, Lo KH, Fasanmade AA, Willerson JT. Randomized, double-blind, placebo- controlled, pilot trial of infliximab, a chimeric monoclo- nal antibody to tumor necrosis factor-alpha, in patients with moderate-to-severe heart failure: results of the anti-TNF Therapy Against Congestive Heart Failure (ATTACH) trial. Circulation. 2003;107:3133-3140.

7. Mann DL, McMurray JJ, Packer M, et al. Targeted antic- ytokine therapy in patients with chronic heart failure: results of the Randomized Etanercept Worldwide Evaluation (RENEWAL). Circulation. 2004;109:1594-1602.

8. Danenberg HD, Fishbein I, Gao J, et al. Macrophage depletion by clodronate-containing liposomes reduces neointimal formation after balloon injury in rats and rabbits. Circulation. 2002;106:599-605.

9. Van Rooijen N, Sanders A. Liposome mediated depletion of macrophages: mechanism of action, preparation of liposomes and applications. J Immunol Methods. 1994;174:83-93.

10. Alves-Rosa F, Stanganelli C, Cabrera J, van Rooijen N, Palermo MS, Isturiz MA. Treatment with liposomeencapsulated clodronate as a new strategic approach in the management of immune thrombocytopenic purpura in a mouse model. Blood. 2000;96:2834-2840.

11. van Amerongen MJ, Harmsen MC, van Rooijen N, Petersen AH, van Luyn MJ. Macrophage depletion impairs wound healing and increases left ventricular remodeling after myocardial injury in mice. Am J Pathol.2007;170:818-829.

12. Dai W, Wold LE, Dow JS, Kloner RA. Thickening of the infarcted wall by collagen injection improves left ventricular function in rats: a novel approach to preserve cardiac function after myocardial infarction. J Am Coll Cardiol. 2005;46:714-719.

13. van den Borne S, Isobe S, Verjans J, et al. Molecular imaging of post-infarction cardiac remodeling and effects of anti-angiotensin therapy. Circulation. 2007;116-II:1409.

14. Torre-Amione G, Kapadia S, Lee J, Bies RD, Lebovitz R, Mann DL. Expression and functional significance of tumor necrosis factor receptors in human myocardium. Circulation. 1995;92:1487-1493.

15. Bilate AM, Salemi VM, Ramires FJ, et al. TNF blockade aggravates experimental chronic Chagas disease cardiomyopathy. Microbes Infect. 2007;9:1104-1113.

16. Huber SA, Feldman AM, Sartini D. Coxsackievirus B3 induces T regulatory cells, which inhibit cardiomyopathy in tumor necrosis factor-alpha transgenic mice. Circ Res. 2006;99:1109-1116.

17. Bettelli E, Oukka M, Kuchroo VK. T(H)-17 cells in the circle of immunity and autoimmunity. Nat Immunol. 2007;8:345-350.

18. Harrington LE, Hatton RD, Mangan PR, et al. Interleu- kin 17-producing CD4p effector T cells develop via a lineage distinct from the T helper type 1 and 2 lineages. Nat Immunol. 2005;6:1123-1132.

19. Kolls JK, Linden A. Interleukin-17 family members and inflammation. Immunity. 2004;21:467-476.

20. Rangachari M, Mauermann N, Marty RR, et al. T-bet negatively regulates autoimmune myocarditis by sup- pressing local production of interleukin 17. J Exp Med. 2006;203:2009-2019. 


\section{CHAPTER 4}

\section{Early and late macrophage depletion in acute myocardial infarction}

H.R. Zandbergen, U.C. Sharma, N. Bitsch, J.W.H. Verjans, J. Slenter, M.E. Kooi, J.G. Maessen, L. Hofstra, J. Narula 


\section{Abstract}

Monocyte macrophage infiltration contributes to infarct healing by removal of necrotic debris and replacement fibrosis in the aftermath of myocardial infarction. The latter evolves secondary to myofibroblastic proliferation and collagen deposition. Expansion in interstitial volume in the remote regions may influence the clinical outcomes adversely. It is hypothesized that modulation of macrophage infiltration may influence replacement and interstitial fibrosis and hence outcome. We have used clodronate liposome administration to selectively deplete macrophages early and late after infarction and evaluate the impact on the evolution of left ventricular function. 
The role of macrophages in wound healing in acute myocardial infarction (MI) has been frequently debated. ${ }^{1,2}$ It has been considered to be a double-edge sword. The macrophage infiltration is deemed necessary to scavenge the necrotic debris from the infarct site, its intimate interaction with myofibroblasts and collagen production is both beneficial and deleterious. Whereas collagen laid down in the infarcted zone provides strength to the scar and restricts infarct expansion, gradual fibrosis in the peri-infarct and remote zones leads to ventricular remodeling. It is therefore important that the role of macrophages is clarified by selective depletion of macrophage infiltration in the infarcted myocardium.

For this purpose, we injected liposomal clodronate (LC) intravenously for selective elimination of macrophages. ${ }^{3,4}$ Plain liposomes (PL) were used as treatment control. Liposomal clodronate is phagocytosed by circulating monocytes. Free clodronate does not cross cell membranes and has an extremely short half-life in the circulation. Liposomal clodronate is removed from the circulation by the renal system and has no major adverse effects on cell types that do not phagocytose the liposomes. ${ }^{3-5}$ In our previous experiment we analyzed peripheral blood monocytes and CD4+ and CD8+ T cells in Ren2 rats at 2 and 7 days after intravenous infusion of LC or PL by flow cytometry. ${ }^{6}$ Rphycoerythrin (RPE)-conjugated anti-CD4, CD8, and CD68 antibodies (Serotec, Dusseldorf, Germany) and isotype controls. Baseline peripheral blood monocyte proportion in PL-treated rats was $2.28 \% \pm 0.15 \%$. Two days after LC injection the number of monocytes sharply reduced to $0.81 \% \pm 0.21 \%$ whereas $\mathrm{CD} 4$ and $\mathrm{CD} 8$ count remained unchanged (CD4: LC, 43.6\% $\pm 3.5 \%$ vs. PL, 45\% $\pm 4.6 \%$; CD8: LC, 5\% $\pm 2.5 \%$ vs. PL, $4.8 \% \pm 1.9 \%, P=N S$ ). However, the monocyte count returned to baseline levels ( $1.8 \pm 0.38)$ by 7 days.

We depleted macrophages in early and late phases of infarct healing with the help of intravenously administered clodronate-liposomes (CL). ${ }^{6}$ The outcome of CL-related effect of macrophage depletion was evaluated by magnetic resonance imaging based left ventricular functional assessment; plain-liposomes $(\mathrm{PL})$ were used in a treatment control group.

Experimental $\mathrm{MI}$ was produced in 12 Wistar rats. Nine animals with $\mathrm{Ml}$ received $\mathrm{CL}$ and the remaining 3 received $\mathrm{PL}$. CL-treated animals were divided in 3 groups based on the length of $\mathrm{CL}$ treatment (Table 1); animals received $\mathrm{CL}$ throughout 3-week follow-up period, only in the first 12 days or only in the last 12 days. $0.2 \mathrm{ml}$ of CL or PL was administered intravenously every other day through the tail vein. In addition to 3 treatment control rats, no manipulation or surgery was performed in 2 animals that were used as disease control animals.

The myocardial healing in the presence of PL resulted in a 50\% MR-verified reduction in LVEF at 3 weeks after MI. If macrophages were depleted all across the healing period i.e. 
for 28 days, the LVEF improved substantially. The EF remained similarly improved if the macrophages were only depleted in the first 12 days after MI but were allowed to recruit macrophages at day 12 . On the other hand only late depletion of macrophages did not restore LV function (Table 1 ).

Table 1. Administration of Clodronate and Empty Liposoms During Different Time Points Post MI, Left Ventricular Ejection Fraction, Left Ventricular End Diastolic Volume, Left Ventricular End Systolic Volume

\begin{tabular}{lllll}
\hline $\begin{array}{l}\text { Group CL and PL } \\
\text { administration }(d)\end{array}$ & $N$ & $\begin{array}{l}\text { LVEF (\%) } \\
\text { (\%, std error) }\end{array}$ & $\begin{array}{l}\text { LVEDV } \\
\text { (std error) }\end{array}$ & $\begin{array}{l}\text { LVESV } \\
\text { (std error) }\end{array}$ \\
\hline no MI & 2 & $52.37 \pm 1.73^{*} \#+\ddagger ¥$ & $0.28 \pm 0.025$ & $0.13 \pm 0.010$ \\
PL 0-21 & 3 & $26.68 \pm 0.85 * \S$ & $0.37 \pm 0.170$ & $0.27 \pm 0.123$ \\
CL 13-21 & 3 & $25.06 \pm 1.82 * \ddagger \S$ & $0.42 \pm 0.032$ & $0.32 \pm 0.021$ \\
CL 0-10 & 3 & $34.49 \pm 2.13^{*}+¥$ & $0.29 \pm 0.038$ & $0.19 \pm 0.032$ \\
CL 0-21 & 3 & $34.91 \pm 1.02 * \#+\ddagger$ & $0.31 \pm 0.019$ & $0.20 \pm 0.010$ \\
\hline
\end{tabular}

Data are presented as mean \pm Standard error

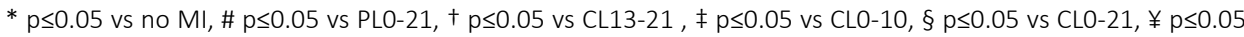
vs CLO-7

In $\mathrm{CL}$ treatment experiments, the functional deterioration was more pronounced when macrophages were present in the initial 2 weeks after infarction and demonstrated improvement if they were depleted early. The results are intriguing, and it is reasonable to suggest that a reduction in macrophage infiltration early after MI may be of some benefit. Although macrophages are necessary for the scavenger function ${ }^{2}$, accompanying cytokine activity may delay the healing process. ${ }^{7}$ Since macrophages are only partially obviated it is conceivable that the partial reduction in macrophage number may not adversely affect interaction with myofibroblasts and collagen deposition. 


\section{References}

1. van den Borne, S. W., S. Isobe, J. W. Verjans, et al. (2008). Molecular imaging of interstitial alterations in remodeling myocardium after myocardial infarction. J Am Coll Cardiol 52(24): 2017-28.

2. van den Borne, S. W., J. Diez, W.M. Blankesteijn, et al. (2010). Myocardial remodeling after infarction: the role of myofibroblasts. Nat Rev Cardiol 7(1): 30-7.

3. Danenberg, H.D., I. Fishbein, J. Gao, et al. (2002). Macrophage depletion by clodronate-containing liposomes reduces neointimal formation after balloon injury in rats and rabbits. Circulation 106:599-605.

4. van Rooijen, N., A. Sanders (1994). Liposome mediated depletion of macrophages: mechanism of action, preparation of liposomes and applications. J Immunol Methods 174:83-93.

5. Alves-Rosa, F., C. Stanganelli, J. Cabrera, et al. (2000). Treatment with liposome- encapsulated clodronate as a new strategic approach in the management of immune thrombocytopenic purpura in a mouse model. Blood 96:2834-2840.

6. Zandbergen, H. R., U. C. Sharma, S. Gupta, et al. (2009). Macrophage depletion in hypertensive rats accelerates development of cardiomyopathy. J Cardiovasc Pharmacol Ther 14(1): 68-75.

7. Frangogiannis, N.G., C.W. Smith, M.L. Entman (2002). The inflammatory response in myocardial infarction. Cardiovasc Research 53(1): 31-47 



\section{CHAPTER 5}

\section{Geometrical models for cardiac MRI in}

rodents: comparison of quantification of left ventricular volumes and function by various geometrical models with a full-volume MRI data set in rodents 


\section{Abstract}

MRI has been proven to be an accurate method for noninvasive assessment of cardiac function. One of the current limitations of cardiac MRI is that it is time consuming. Therefore, various geometrical models are used, which can reduce scan and postprocessing time. It is unclear how appropriate their use is in rodents. Left ventricular (LV) volumes and ejection fraction (EF) were quantified based on 7.0 Tesla cine-MRI in 12 wild-type (WT) mice, 12 adipose triglyceride lipase knockout $\left(\mathrm{ATGL}^{-/-}\right)$mice (model of impaired cardiac function), and 11 rats in which we induced cardiac ischemia. The LV volumes and function were either assessed with parallel short-axis slices covering the full volume of the left ventricle (FV, gold standard) or with various geometrical models [modified Simpson rule (SR), biplane ellipsoid (BP), hemisphere cylinder (HC), singleplane ellipsoid (SP), and modified Teichholz Formula (TF)]. Reproducibility of the different models was tested and results were correlated with the gold standard (FV). All models and the FV data set provided reproducible results for the LV volumes and EF, with interclass correlation coefficients $\geq 0.87$. All models significantly over- or underestimated $E F$, except for SR. Good correlation was found for all volumes and EF for the SR model compared with the FV data set $\left(R^{2}\right.$ ranged between $0.59-0.95$ for all parameters). The $\mathrm{HC}$ model and BP model also predicted EF well $\left(R^{2} \geq 0.85\right)$, although proved to be less useful for quantitative analysis. The SP and TF models correlated poorly with the FV data set $\left(R^{2} \geq 0.45\right.$ for $E F$ and $R^{2} \geq 0.29$ for $E F$, respectively). For the reduction in acquisition and postprocessing time, only the SR model proved to be a valuable method for calculating LV volumes, stroke volume, and EF.

Cardiovascular diseases still are one of the main causes of death in Western countries, and the incidence and prevalence of cardiovascular diseases are still increasing $(28,29)$. Therefore, many studies have focused on the prevention, treatment, and etiology of these diseases. Rodent models of cardiac disease have played a critical role in this area of expertise and hence models to assess rodent cardiac function have become increasingly important over the last decades $(12,24)$. MRI has been proven to be an accurate method for noninvasive assessment of cardiac function in rodents $(22,23,25)$ and has therefore become an important tool in studies of rodent models of cardiac disease $(7,14)$. With MRI, systolic function can be assessed by calculating the ejection fraction (EF) from sequential multislice short-axis cine-MRI covering the complete volume of the left ventricle (25). However, this requires an acquisition of at least six to eight slices (depending on the size of the heart), which can be quite time consuming, especially in studies requiring high spatial resolution. Also manual or semiautomatic analysis of these slices can demand a substantial amount of time in high-throughput applications (31). Furthermore, it might be desirable to be able to allow quick assessment of the cardiac function, as a secondary parameter, without increasing scan time too much. The acquisition duration is not only demanding in terms of scan time but also prolongs the duration of anesthesia and hence increases stress in the animals under investigation $(5,11)$. In 
specific cardiovascular compromised genotypes, such as the adipose triglyceride lipase knockout $\left(\mathrm{ATGL}^{-/}\right)$mouse, which was used in our studies (10), prolonged anesthesia can in some cases increase mortality rate.

To circumvent this, several studies have implemented geometrical models for assessment of cardiac function in rodents, rather than acquiring multiple parallel slices with multislice cine-MRI to determine the full volume of the left ventricle (4, $8,13,15,18,26,30)$. These models estimate left ventricular (LV) volumes based on single or perpendicular biplane or even triplane slices (6) and therefore allow quick assessment of $L V$ volumes and $E F$, as they only require acquisition of one up to three instead of six to eight slices for mice. For rats, due to the larger size of the left ventricle, the reduction in number of slices is even larger, i.e., 1-3 instead of 6-10 slices. This would substantially reduce total scan time with 10-30 min for mice and even 20-45 min for rats. A typical total examination time during which the mice and rats are kept under anesthesia for assessment of cardiac function is $40-45$ and $45-60 \mathrm{~min}$, respectively. Additionally, acquisition of images required for the geometrical models instead of a full volume data set would reduce the number of slices to be segmented to calculate the LV volumes and EF with a factor of 2-3 for mice and 2-4 for rats, thereby also reducing time spent on the assessment of cardiac function in rodent models of cardiac disease.

Although these models have been compared with ultrasound (4) and have been validated in humans (6), to our knowledge no study has determined which of these geometrical models is most accurate in determining cardiac function compared with the gold standard, i.e., cine-MRI of the complete left ventricle by full volume imaging in rodents. Therefore, the aim of the present study was to evaluate reproducibility and validity of these geometrical models in mice compared with the gold standard, cine-MRI of the complete left ventricle. After testing the validity of these geometrical models in wild-type mice with anticipated normal cardiac function, we tested applicability of these models in mice with anticipated cardiac failure and in rats in which we induced myocardial infarction.

\section{Methods}

\section{Animals}

Mice and rats were housed under standard conditions at $25^{\circ} \mathrm{C}$ with a $14: 10$-h light-dark cycle with ad libitum access to water and standard chow diet. The Maastricht University Medical Centre Institutional Ethics Committee on Animal Welfare approved all experiments. Twelve wild-type C57BL/6 mice with anticipated normal cardiac function were imaged for validation of the geometrical models. In addition, we extended the validation to a model of severe cardiac dysfunction by using 12 adipose triglyceride lipase knockout mice ( $\mathrm{ATGL}^{-/-}$, age 8-12 wk). ATGL ${ }^{-/-}$mice were generated on a mixed genetic back- 
ground (50\% C57BL/6 and 50\% 129/Ola) as previously described (9). The targeted ATGL allele was then backcrossed onto the $\mathrm{C} 57 \mathrm{BL} / 6$ background strain for $>10$ generations. Furthermore, myocardial infarction (MI) was induced in 11 male Wistar-rats (300-500 g) by ligation of the left anterior descending coronary artery using a 6-0 Prolene suture, as described previously $(17,19)$. Subsequently, the rats were scanned between 7 and 21 days post-MI.

Before MRI, mice were anesthetized using 1-2\% isoflurane (Abbott Laboratories, Queensborough, UK) in medical air. Neonatal ECG electrodes (3M, St. Paul, MN) were placed on the paws of the right front leg and left hind leg and connected to an MR compatible small animal monitoring system (SA Instruments, Stony Brook, NY). Animals were placed on a warm waterbed. Respiratory rate was continuously monitored.

\section{MRI protocol}

MRI was performed on a 7 Tesla Bruker Biospec 70/30 USR (Bruker Biospin, Ettlingen, Germany) using the BGA12-S mini-imaging gradient (maximum gradient strength: 400 $\mathrm{mTm}^{-1}$, slew rate: $5,000 \mathrm{Tm}^{-1} \cdot \mathrm{s}^{-1}$, and linear inductive rise time: 5-95\% on all axis, $80 \mu \mathrm{s}$ ), interfaced to an AVANCE II console. First, a bright blood cine image with 10 cardiac phases was recorded in horizontal 4-chamber view $(4 \mathrm{CH})$ using a retrospectively selfgated protocol (IntraGate, Bruker Biospin; imaging time: $\approx 2-4 \mathrm{~min}$, for the mice: $2.56 \times$ $2.56 \mathrm{~cm}^{2}$ field of view, $164 \times 164$ matrix size; for the rats: $5.0 \times 5.0 \mathrm{~cm}^{2}$ field of view, 128 $\times 128$ matrix size see Fig. $1, /$ and $J)$. This $4 \mathrm{CH}$ orientation was used to plan a long axis view (LA) perpendicular to the $4 \mathrm{CH}$ view with the same self-gated protocol (see Fig. 1, $A$, $B, E$, and $F$ ). The orientation was defined as a sagittal plane through the mitral valve and the apex. Perpendicular to this $L A$ and $4 \mathrm{CH}$ view and the septum, a short axis view (SA) was positioned (see Fig. 1, $C, D, G, H, K$, and $L$ ). The slice was planned at half height of the left ventricle, 1-2 $\mathrm{mm}$ below the leaflets of the mitral valve. This image was acquired ECG and respiratory gated, using a bright blood gradient echo sequence with the following parameters for the mice studied: repetition time: $11.6 \mathrm{~ms}$, echo time: $2.5 \mathrm{~ms}$, flip angle: $50^{\circ}$, one slice, $1-\mathrm{mm}$ thickness, eight signal averages, field of view: $25.6 \times 25.6$ $\mathrm{mm}$, and matrix-size: $164 \times 164$, resulting in an in-plane resolution: $0.16 \times 0.16 \mathrm{~mm}^{2}$ and acquisition time: $\approx 4 \mathrm{~min}$. The SA images of the rats were acquired with the following parameters: repetition time: $7 \mathrm{~ms}$, echo time: $2.8 \mathrm{~ms}$, flip angle: $50^{\circ}, 1$ slice, $1.263-\mathrm{mm}$ thickness, one signal average, field of view: $50 \times 50 \mathrm{~mm}$, and matrix-size: $192 \times 192$, resulting in an in-plane resolution: $0.26 \times 0.26 \mathrm{~mm}^{2}$ and acquisition: time $\approx 5 \mathrm{~min}$. For image analysis of the complete LV volumes, five to nine additional slices were planned parallel to the short axis slice with a slice thickness of $1 \mathrm{~mm}$ in the mice and $1.2 \mathrm{~mm}$ in the rats, without gap between slices. The slices covered the whole left ventricle, from the apex to the base. The images were acquired using the same pulse sequence. The average time for acquiring the images needed to assess EF with a full volume data, using eight slices, is $\approx 50 \mathrm{~min}$. 

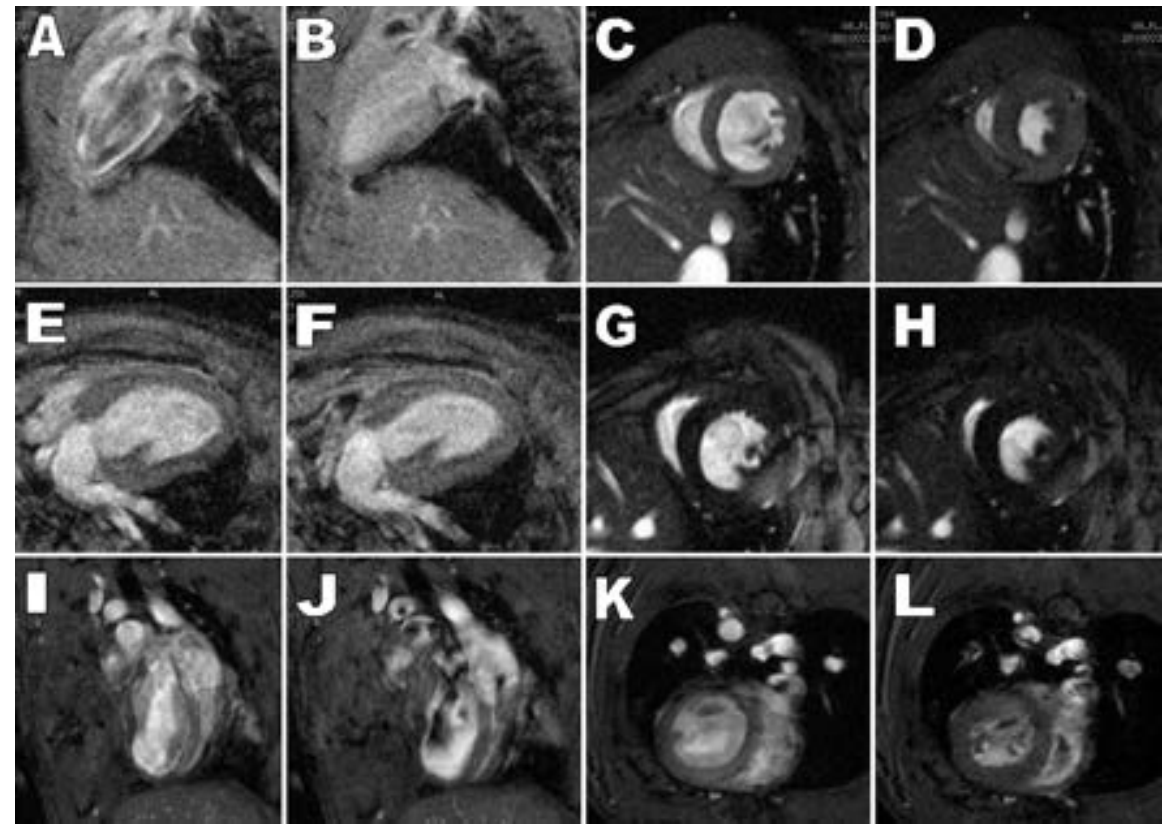

Fig. 1. Long axis (LA), 4-chamber view $(4 \mathrm{CH})$, and short axis (SA) images of adipose triglyceride lipase knockout mice $\left(\mathrm{ATGL}^{-/-}\right.$) and wild-type (WT) mice and a rat model of myocardial infarction. Depicted are the LA-plane images of the WT mice. A: in diastole. B: in systole. E: LA-plane image in diastole of a ATGL ${ }^{-/-}$mice. F: LA-plane image in a $\mathrm{ATGL}^{-/-}$mice in systole. Here a plane through the mitral valve and the apex was chosen to assess left ventricular (LV) length and volume. I: $4 \mathrm{CH}$ view is depicted of a rat heart in diastole. J: 4CH view of a rat heart in diastole, after myocardial infarction. $C$ and $D$ : SA images are depicted in wild-type (WT) mice. $G$ and $H$ : SA images are depicted of a $\mathrm{ATGL}^{-/-}$mice. $K$ and $L$ : SA images are depicted of a rat heart after myocardial infarction in diastole and systole, respectively. SA view was positioned perpendicular to the LA plane and 4CH view for the quantification of LV volumes. Furthermore, in these images an impaired systolic contraction and a dilated and thickened ventricular wall is visible in the $\mathrm{ATGL}^{-/-}$mice (middle), as well as an infracted area in the left ventricle and slight dilatation of the rat heart (bottom).

\section{Image analysis}

All images were analyzed in Osirix (Dicom viewer, version 3.5; Pixmeo, Geneva, Switzerland). The end-diastolic volumes (EDV) and end-systolic volumes (ESV) of the left ventricle were considered the largest and the smallest areas, respectively, of the LV cavity in each slice. For the analysis of cine-MRI, the window width and level were manually adjusted to recognize the internal ventricular morphologic characteristics. For the measurements of LV volumes, the whole LV cavity was selected with semiautomated segmentation parameters in OsiriX. The papillary muscle was excluded from the LV volume during analysis. The diameter $(D)$ of the LV volume on the short axis was measured as the longest distance between the septum and the ventricular wall. Length $(L)$ of the LV on the LA view was defined as the longest distance from the apex to the valves. On average, the time needed to analyze one slice was $3 \mathrm{~min}$. 


\section{Data analysis}

In all animals, EDV, ESV, stroke volume (SV = EDV - ESV), and EF (EF = SV/EDV) were calculated from the EDV and ESV volumes based on either the multislice short axis cineimages of the complete left ventricle (gold standard) or based on the different geometrical models as described by Dulce et al. 1993 (6). The algorithms and required imaging slices for these models can be found in Fig. 2 .

\section{Full volume data set (FV)} $\mathrm{LWV}=\mathrm{S}_{1}+\mathrm{S}_{2}+\mathrm{S}_{3}+\ldots$

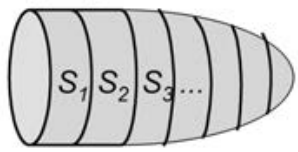

Biplane Ellipsoid model (BP) $\operatorname{LV}=\pi / 6 \times L \times\left(4 / \pi \times A_{m} / D\right) \times\left(4 / \pi \times A_{i} / L\right)$

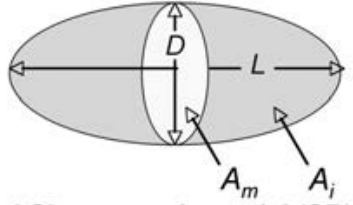

Modified Simpson rule model (SR) $\mathrm{LVV}=A_{m} \times L / 3+\left(A_{m}+A_{p}\right) / 2 \times L / 3+1 / 3 \times A_{p} \times L / 3$

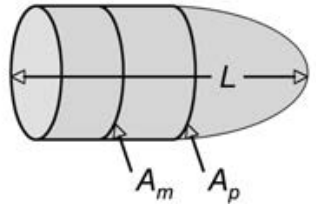

Hemisphere cylinder model (HC)

$\operatorname{LV}=A_{m} \times L / 2+2 / 3 \times A_{m} \times L / 2=5 / 6 \times A_{m} \times L$

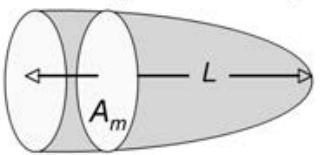

Single-plane Ellipsoid model (SP)

$\operatorname{LW}=\left(8 \times A_{i} \times A_{i}\right) /(3 \pi \times L)=0.85 \times\left(A_{i} \times A_{i}\right) / L$

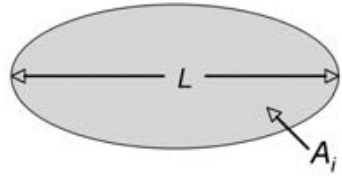

Modified Teichholz Formula (TF) $\mathrm{LW}=[7 /(2.4+D)] \times D \times D \times D$

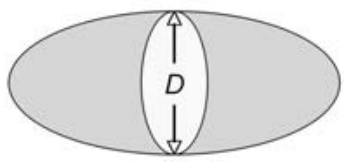

Fig. 2. Algorithms and formula's for calculation of LV volumes with a full volume MRI data set of the complete left ventricle or geometrical models based on a few MRI slices. Top: algorithms for full volume data set and the various geometrical models for the determination of LV volumes and ejection fraction (EF) are presented. $A i$, cross-sectional area of LV cavity in the LA plane; $A m$, cross-sectional area of the LV cavity in the SA plane, $\approx 1-2$ $\mathrm{mm}$ below the mitral valve; $A p$, cross-sectional area of the LV cavity in the SA plane, approximately at the base of the papillary muscles; $D$, diameter of the LV cavity in the short axis plane, $\approx 1-2 \mathrm{~mm}$ below the mitral valve; $L$, longest length of LV cavity in the LA plane; LVV, LV volumes [end-diastolic volume (EDV) and end-systolic volume (ESV), respectively]; S1, S2, S3, .., 1-mm SA slices from the apex to the base of the left ventricle.

\section{Statistical analysis}

Measurements of LV volumes and EF from cine MRI in both the wild type animals and the transgenic $\mathrm{ATGL}^{-/-}$mice are presented as mean values $\pm \mathrm{SD}$. The correlations of LV volumes and $E F$, as measured with the various geometric models vs. the full volume data set of the left ventricle (gold standard), were assessed by linear regression analysis. $R^{2}, P$ values, and 95\% individual confidence intervals (also known as prediction intervals) are reported for all regression analysis. Differences in LV volumes and EF between the geometric models and the gold standard were analyzed for statistical significance with a 
one-way ANOVA. Also, differences in outcome measures between the different genotypes were assessed with a one-way ANOVA for each model separately. $P$ value $<0.05$ was considered statistically significant. Reproducibility was tested by calculating the interclass correlation for two consecutive measurements for all geometrical models and the gold standard in five wild-type mice. For measuring the two consecutive measurements, mice were taken out of the scanner in-between the measurements, where after they were repositioned and all preparatory steps (including shimming and acquisition of scout-images) were repeated.

\section{Results}

\section{Reproducibility}

The interclass correlation coefficients for each model for the LV volumes and EF are summarized inTable 1 . The interclass correlation coefficient was $>0.85$ for all parameters in each model indicating a good reproducibility for all models.

Table 1. Interclass correlation coefficients of LV volumes and ejection fraction in wild-type animals

\begin{tabular}{lllll}
\hline \multicolumn{5}{c}{ Wild-type animals-interclass correlation coefficients } \\
\cline { 2 - 4 } & EDV & ESV & SV & EF \\
\hline FV & 0.944 & 0.915 & 0.981 & 0.995 \\
BP & 0.991 & 0.994 & 0.986 & 0.975 \\
SR & 0.995 & 0.981 & 0.986 & 0.955 \\
HC & 0.979 & 0.997 & 0.961 & 0.996 \\
SP & 0.997 & 0.989 & 0.976 & 0.939 \\
TF & 0.983 & 0.893 & 0.887 & 0.866 \\
\hline
\end{tabular}

Values were obtained with geometric models and a complete imaging set of the left ventricle (LV) acquired with cine-MRI. Presented are the interclass correlation coefficients of a repeated measurement in 5 mice. EDV and ESV, end-diastolic and end-systolic volume; SV, stroke volume; EF, ejection fraction; FV, full volume data set; BP, biplane ellipsoid model; SR, modified Simpson rule model; HC, hemisphere cylinder model; SP, single plane ellipsoid model; TF, modified Teichholz formula model.

\section{Validation of $L V$ volumes and $E F$}

The values for LV volumes and EF in the various animal groups are summarized in Table 2. Only the modified Simpson rule model gave statistically indifferent values for both the LV volumes and the EF compared with the full volume data set for all study groups. All models could pick up the marked differences in LV volumes and EF found between wildtype and transgenic mice ( $P<0.05$, one-way ANOVA test, data not shown). 
Table 2. LV volumes and EF

\begin{tabular}{|c|c|c|c|c|}
\hline & EDV, $\mu l$ & ESV, $\mu \mathrm{l}$ & SV, $\mu l$ & $E F, \%$ \\
\hline \multicolumn{5}{|c|}{ Wild-type mice $(n=12)$} \\
\hline FV & $52.7 \pm 3.3$ & $14.8 \pm 1.0$ & $37.9 \pm 2.8$ & $71.5 \pm 1.5$ \\
\hline $\mathrm{BP}$ & $84.8 \pm 6.0^{*}$ & $18.0 \pm 1.8(\mathrm{NS})$ & $66.8 \pm 4.6^{*}$ & $79.0 \pm 1.1^{*}$ \\
\hline SR & $60.3 \pm 2.7$ (NS) & $14.7 \pm 1.4$ (NS) & $45.6 \pm 2.1$ (NS) & $75.9 \pm 1.8$ (NS) \\
\hline $\mathrm{HC}$ & $71.2 \pm 3.6^{\dagger}$ & $14.3 \pm 1.4$ (NS) & $56.9 \pm 2.7^{*}$ & $80.2 \pm 1.3^{*}$ \\
\hline $\mathrm{SP}$ & $58.0 \pm 5.0(\mathrm{NS})$ & $22.2 \pm 2.2$ (NS) & $35.9 \pm 3.7$ (NS) & $61.4 \pm 2.6^{*}$ \\
\hline $\mathrm{TF}$ & $149.5 \pm 15.5^{*}$ & $45.4 \pm 7.5^{*}$ & $104.1 \pm 9.6^{*}$ & $71.1 \pm 3.0(\mathrm{NS})$ \\
\hline \multicolumn{5}{|c|}{ Transgenic mice $(n=12)$} \\
\hline FV & $53.4 \pm 2.7$ & $28.6 \pm 3.3$ & $20.6 \pm 3.8$ & $47.6 \pm 4.2$ \\
\hline $\mathrm{BP}$ & $89.3 \pm 4.9^{*}$ & $41.0 \pm 5.8$ (NS) & $48.3 \pm 3.3^{*}$ & $55.9 \pm 4.9(\mathrm{NS})$ \\
\hline SR & $55.0 \pm 4.8(\mathrm{NS})$ & $27.1 \pm 4.7$ (NS) & $27.9 \pm 1.6(N S)$ & $53.4 \pm 4.8(\mathrm{NS})$ \\
\hline $\mathrm{HC}$ & $74.2 \pm 5.1$ (NS) & $35.2 \pm 4.9$ (NS) & $39.1 \pm 2.2^{*}$ & $54.7 \pm 4.2(\mathrm{NS})$ \\
\hline $\mathrm{SP}$ & $63.8 \pm 5.7(\mathrm{NS})$ & $48.0 \pm 7.0$ (NS) & $15.8 \pm 2.1(\mathrm{NS})$ & $28.6 \pm 5.2^{*}$ \\
\hline \multirow[t]{2}{*}{$\mathrm{TF}$} & $180.8 \pm 20.3^{*}$ & $135 \pm 18.6^{*}$ & $45.8 \pm 9.6^{*}$ & $26.8 \pm 4.7^{*}$ \\
\hline & $\mathrm{EDV}, \mathrm{ml}$ & $\mathrm{ESV}, \mathrm{ml}$ & $\mathrm{SV}, \mathrm{ml}$ & $E F, \%$ \\
\hline \multicolumn{5}{|c|}{ Rat ischemic heart model $(n=11)$} \\
\hline FV & $0.36 \pm 0.05$ & $0.23 \pm 0.04$ & $0.13 \pm 0.02$ & $35.2 \pm 5.7$ \\
\hline $\mathrm{BP}$ & $0.36 \pm 0.07$ (NS) & $0.24 \pm 0.06(\mathrm{NS})$ & $0.12 \pm 0.04$ (NS) & $33.6 \pm 11.9$ (NS) \\
\hline $\mathrm{SR}$ & $0.36 \pm 0.05$ (NS) & $0.23 \pm 0.04$ (NS) & $0.13 \pm 0.03$ (NS) & $36.1 \pm 6.4$ (NS) \\
\hline $\mathrm{HC}$ & $0.33 \pm 0.05^{+}$ & $0.21 \pm 0.05$ (NS) & $0.11 \pm 0.03$ (NS) & $35.4 \pm 7.8(N S)$ \\
\hline $\mathrm{SP}$ & $0.24 \pm 0.11^{*}$ & $0.17 \pm 0.11^{*}$ & $0.07 \pm 0.03^{*}$ & $34.7 \pm 18.4(\mathrm{NS})$ \\
\hline $\mathrm{TF}$ & $0.39 \pm 0.29$ (NS) & $0.23 \pm 0.17$ (NS) & $0.16 \pm 0.16(N S)$ & $41.3 \pm 23.6^{\dagger}$ \\
\hline
\end{tabular}

Presented above are the averages and SD of the LV volumes and EF \pm SD per group (wild-type mice, adipose triglyceride lipase knockout mice $\left(\mathrm{ATGL}^{-/-}\right.$) mice, and rats with cardiac ischemia) and per geometrical model. Values were obtained with geometric models and the complete imaging set of the entire left ventricle acquired with cine-MRI. * $P<0.05 ;+P<0.10$; NS, not significant, when compared with the gold standard (FV).

\section{Linear regression analysis}

The scatter plots with individual regression lines of the EF quantified by the models vs. the gold standard (quantification based on full volume data set) are provided in Fig. 3 . Pearson's correlation coefficients $(r)$, individual 95\% confidence intervals and $R^{2}$ can also be found in Fig. 3. As the modified Simpson rule model was the only model that provided the correct absolute values for EF and LV volumes, we also present the scatter plots of the LV volumes and SV with the full volume data set for this model in Fig. 4 (for mice and rats the data are presented separately). 
A

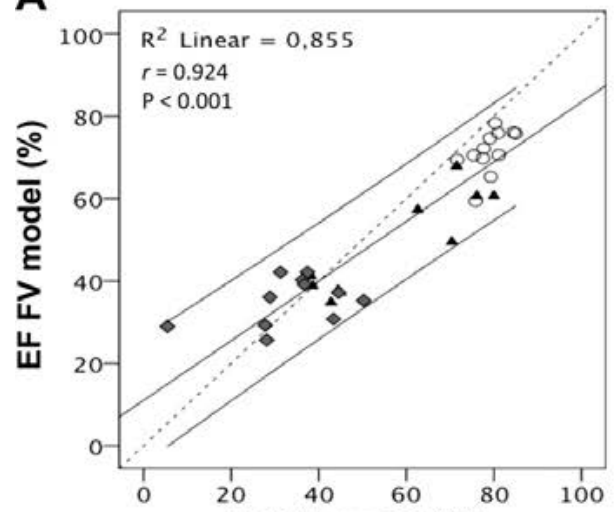

C

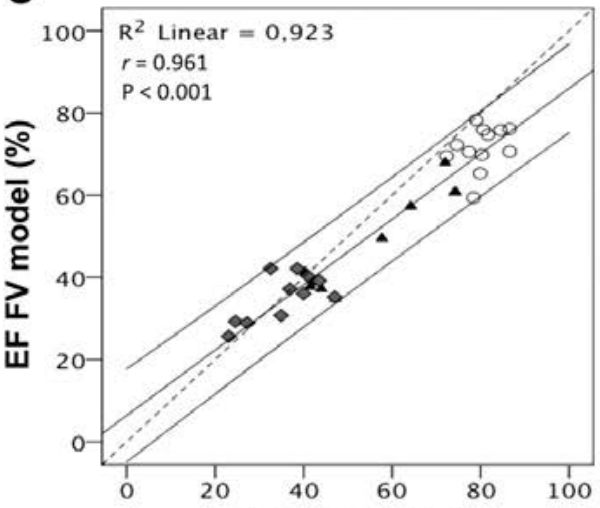

E

EF HC model (\%)

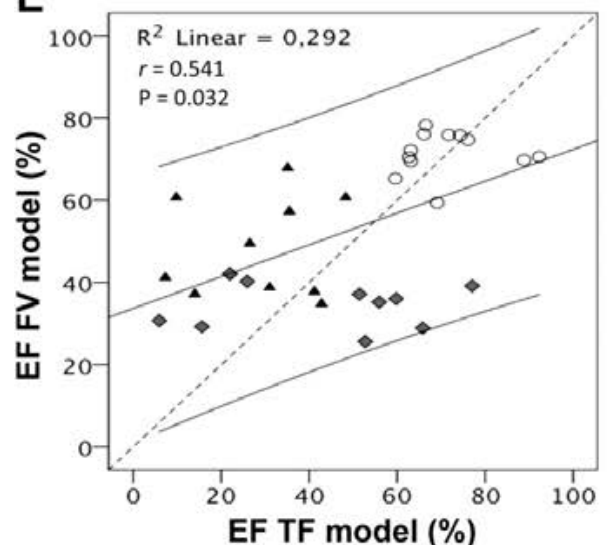

B

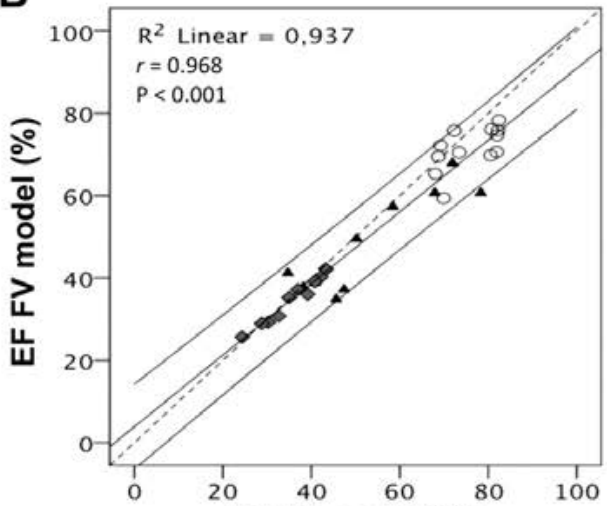

EF SR model (\%)

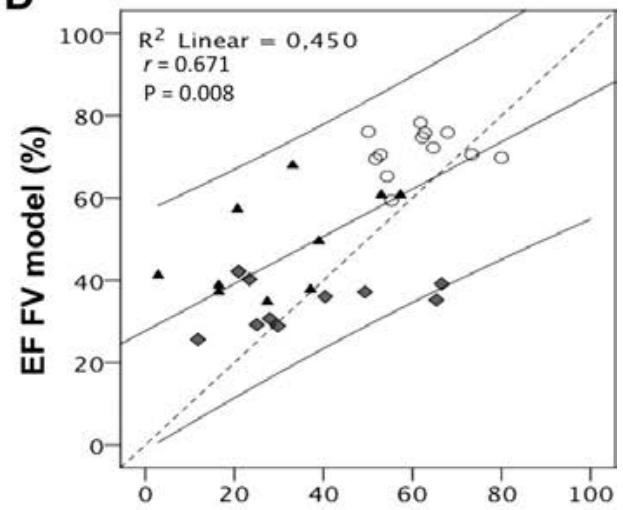

EF SP model (\%)

Fig. 3. Scatter plots of EF. Scatter plots of EFs of the various models vs. full volume data set for $A$. B: biplane ellipsoid model. $C$ : modified Simpson rule model. $C$ : hemisphere cylinder model. $D$ : single plane ellipsoid model. E: modified Teichholz Formula. In all scatter plots, the regression line is given with a $95 \%$ confidence interval. $R$ squares and Pearson correlation coefficients (with according $P$ values) can also be found in the graph for each fit. 

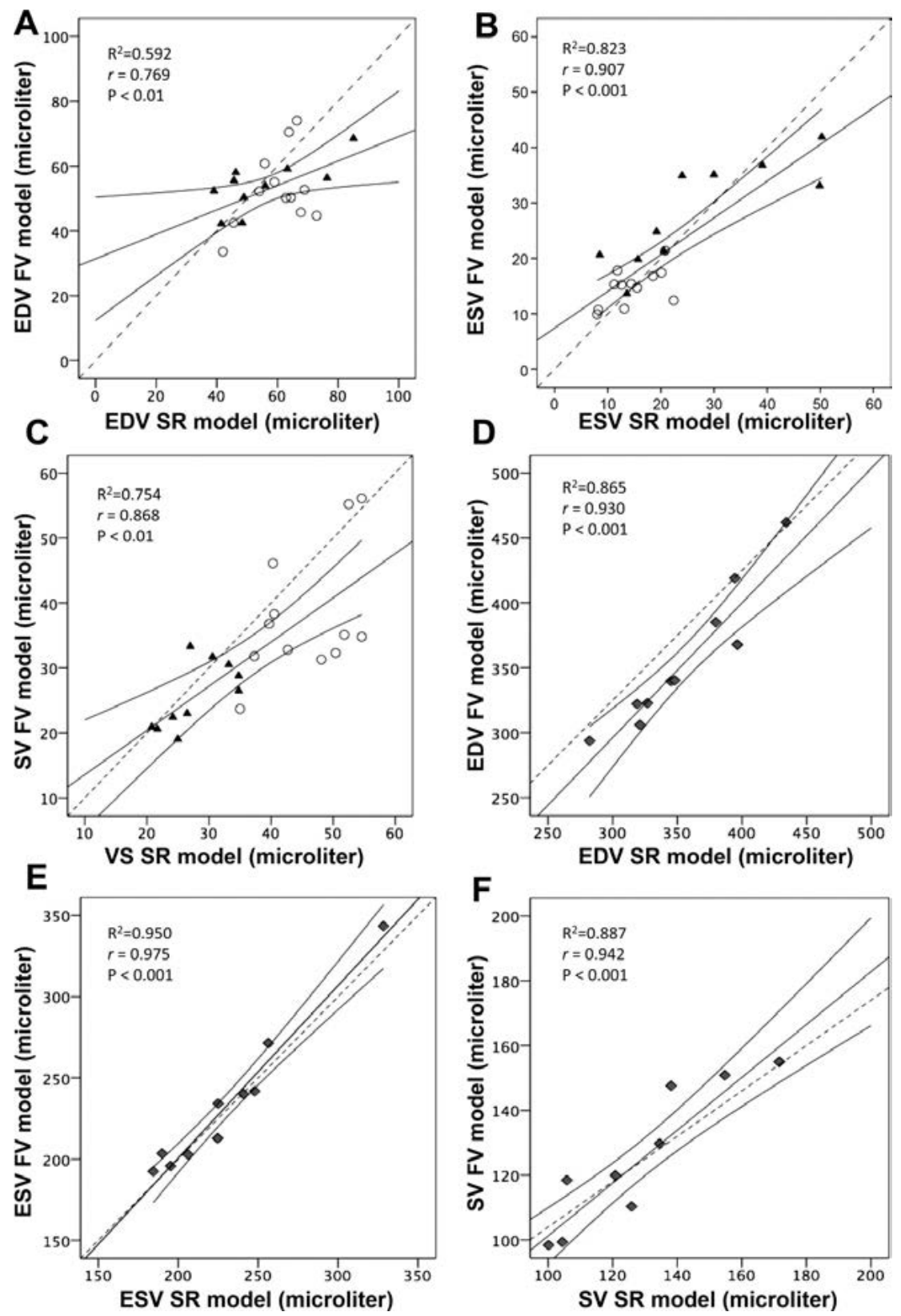

Fig. 4. Scatter plots of LVV for the modified Simpson rule vs. the full volume data set. Scatter plots of LVV and stroke volume for the modified Simpson rule model (SR) vs. full volume data set. $A$ : scatter plot for the EDV in WT (O) and $\mathrm{ATGL}^{-/-}(\mathbf{\Delta})$ mice. B: scatter plot for the ESV in WT and $\mathrm{ATGL}^{-/-}$mice. C: scatter plot for the stroke volumes in $\mathrm{WT}$ and $\mathrm{ATGL}^{-/-}$mice. D: scatter plot for the EDV in rats with myocardial infarction (MI) ( $\left.\downarrow\right)$. E: scatter plot for the ESV in rats with MI. F: scatter plot for the stroke volumes in rats with MI. In all scatter plots, the regression line is given with a 95\% confidence interval. Dotted line in the graph represents $x=y$. $R$ squares and Pearson correlation coefficients (with according $P$ values) can also be found in the graph for each fit. 
Although all correlations were significant, only the hemisphere cylinder model, the modified Simpson rule model and the biplane ellipsoid model showed a linear regression with a Pearson's correlation coefficient for EF $>0.85$. The other two models showed a poor correlation with correlation coefficients $<0.7$ and $R^{2}$ values $<0.45$. The $95 \%$ confidence interval was smallest for the modified Simpson rule model, as the $R^{2}$ and $r$-values were the highest for this model $\left(R^{2}=0.937, r=0.968\right.$, and $\left.P<0.001\right)$. The LV volumes and SV, as calculated with the modified Simpson rule model, correlated with the full volume data set both in the mouse models as well as in the rat model. However, it should be noticed that the correlations were stronger for the rat model. The $R^{2}$ values ranged between $0.59-0.95$ with $P$ values $<0.01$ for all correlations (also see Fig. 4).

\section{Discussion}

In this study, we aimed to assess the validity of common geometrical models compared with the gold standard, a full volume data set, for quantification of LV volumes and EF with cine-MRI in rodents. We showed that the reproducibility of all models was good, with higher variations in the Teichholz formula model. When the models were compared with the full volume data set, only the modified Simpson rule model showed no significant differences with the gold standard for all the calculated parameters. The values found for the LV volumes, SV, and EF with the modified Simpson rule model correlated well with the full volume data set (gold standard). Also the EF found with the hemisphere cylinder and biplane ellipsoid model showed high correlation coefficients with the full volume data set. The two remaining models were poor predictors of EF.

Although these findings are new for MRI applications, previous research using other imaging modalities already pointed in this direction. For instance, it has been shown that the Teichholz formula model does not give comparable results in MRI compared with ultrasound (4). These results also did not correlate with values found with the modified Simpson rule model in this study either (4). The discrepancy in absolute values compared with the gold standard for all models except the modified Simpson rule points out that care should be taken when cardiac function is compared between studies using different methodology.

By using the modified Simpson rule, one needs to acquire and assess only three imaging planes, thereby reducing total examination time by $10-45 \mathrm{~min}$ compared with total examination time including positioning of the animal of 30-60 min in the present study. In addition, segmentation time is reduced by two to threefold (depending on the animal species used). The modified Simpson rule model is the only model that divides the heart into three different compartments, based on three imaging planes. This may explain why it is more accurate in estimating the LV volumes, and therefore it might also be more sensitive for nonsymmetrical pathologies (like myocardial infarction, for instance). The other models are based on only one (modified Teichholz formula and single 
plane ellipsoid model) or two (Hemisphere and biplane ellipsoid models) imaging planes. The most simplified models, using only one plane, showed indeed the weakest correlations, whereas the two plane models did better. Although the EF as determined by these two plane models correlated well with the EF calculated from the full volume data set, they significantly over- or underestimated the real volumes of the left ventricle. Therefore, only the modified Simpson rule can be applied in rodent studies of cardiac disease.

For high throughput studies, parallel imaging or simultaneous scanning of multiple animals might by used as an alternative to reduce scan time. These methods will, however, not lead to a reduction in segmentation time. Scanning multiple animals simultaneously is still a quite new technique, which requires specific system requirements (1-3). Parallel imaging leads to a reduction of the signal-to-noise ratio and therefore may not always be appropriate $(16,20,21,27)$. The Simpson rule model could be a good alternative, and combining parallel imaging with the Simpson rule model will even further increase the throughput.

One of the limitations of this study is that the mouse model of transient cardiac disease, namely the $\mathrm{ATGL}^{-/-}$as a model for cardiomyopathy, is quite a rare model. Nonetheless, we choose to use this model as their cardiac function is severely impaired and the progression of their disease is rather extreme (9), which introduces variation in the main outcome parameters as can be found in Table 2. This variation is not due to a poor reproducibility in this specific group, rather it is due to variation of the disease stage as cardiac function drastically drops with age. A greater variation in the outcome parameters gives a larger spread in the data and makes these data more suited for linear regression analysis. Therefore, this model was ideal for looking at correlations in EF. In addition to the $\mathrm{ATGL}^{-/-}$mice, we also included wild-type mice and rats with myocardial infarction in the present study, since these are more commonly used.

Introducing the rat model of myocardial infarction in this study not only introduced a more common model of cardiac disease but also increased the number of animals for linear regression analysis of EF. One drawback might be that we did handle two different kinds of species in the same analysis. Therefore, we analyzed the correlations between the modified Simpson rule model and full volume data set for the LV volumes and SV for the different animal species separately. The LV volumes seemed to correlate better in the rat model than in the mouse model. The differences in the correlations found are most likely due to the better estimation of the LV volumes in the segmentation analysis of the rat hearts due to relatively higher resolution of the left ventricle (as the LV is quite a bit bigger in rats). Also, the spread of the EDV in the rat hearts was greater, resulting in a better spread of the data suitable for regression analysis.

In conclusion, the modified Simpson rule is a good alternative to a full volume data set and reduces scan and postprocessing time. Comparing the absolute values as determined by different geometrical models should be done with caution as they give variable results. 


\section{Grants}

This work was supported by the Center for Translational Molecular Medicine and the Netherlands Heart Foundation, Dutch Diabetes Research Foundation, and Dutch Kidney Foundation (PREDICCt). V. B. Schrauwen-Hinderling is supported by a VENI grant from the Netherlands Organisation for Scientific Research (NWO).

\section{Disclosures}

No conflicts of interest, financial or otherwise, are declared by the author(s).

Copyright (C) 2012 the American Physiological Society 


\section{References}

1. Bishop J, Feintuch A, Bock NA, Nieman B, Dazai J, Davidson L, Henkelman RM . Retrospective gating for mouse cardiac MRI. Magn Reson Med 55: 472-477, 2006.

2. Bock NA, Konyer NB, Henkelman RM . Multiple-mouse MRI. Magn Reson Med 49: 158-167, 2003.

3. Bock NA, Nieman BJ, Bishop JB, Henkelman MR. In vivo multiple-mouse MRI at 7 Tesla. Magn Reson Med54: 1311-1316, 2005.

4. Bunck AC, Engelen MA, Schnackenburg B, Furkert J, Bremer C, Heindel W, Stypmann J, Maintz D .Feasibility of functional cardiac MR imaging in mice using a clinical 3 Tesla whole body scanner. Invest Radiol44: 749-756, 2009.

5. Cottrell JE . We care, therefore we are: anesthesia-related morbidity and mortality: the 46th Rovenstine Lecture. Anesthesiology 109: 377-388, 2008.

6. Dulce MC, Mostbeck GH, Friese KK, Caputo GR, Higgins CB . Quantification of the left ventricular volumes and function with cine MR imaging: comparison of geometric models with three-dimensional data. Radiology188: 371-376, 1993.

7. Epstein FH . MR in mouse models of cardiac disease. NMR Biomed 20: 238-255, 2007.

8. Franco F, Dubois SK, Peshock RM, Shohet RV. Magnetic resonance imaging accurately estimates LV mass in a transgenic mouse model of cardiac hypertrophy. Am J Physiol Heart Circ Physiol 274: H679H683,1998.

9. Haemmerle G, Lass A, Zimmermann R, Gorkiewicz G, Meyer C, Rozman J, Heldmaier G, Maier R, Theussl C, Eder S, Kratky D, Wagner EF, Klingenspor M, Hoefler G, Zechner R . Defective lipolysis and altered energy metabolism in mice lacking adipose triglyceride lipase. Science 312: 734-737, 2006.

10. Haemmerle G, Moustafa T, Woelkart G, Buttner S, Schmidt A, van de Weijer T, Hesselink M, Jaeger D, Kienesberger PC, Zierler K, Schreiber R, Eichmann T, Kolb D, Kotzbeck P, Schweiger M, Kumari M, Eder S, Schoiswohl G, Wongsiriroj N, Pollak NM, Radner FP, Preiss-Landl K, Kolbe T, Rulicke T, Pieske B, Trauner M, Lass A, Zimmermann R, Hoefler G, Cinti S, Kershaw EE, Schrauwen P, Madeo F, Mayer B, Zechner R . ATGL-mediated fat catabolism regulates cardiac mitochondrial function via PPAR-alpha and PGC-1. Nat Med 17:1076-1085.

11. Hanusch C, Hoeger S, Beck GC. Anaesthesia of small rodents during magnetic resonance imaging.Methods 43: 68-78, 2007.

12. Hasenfuss $\mathrm{G}$. Animal models of human cardiovascular disease, heart failure and hypertrophy.Cardiovasc Res 39: 60-76, 1998.

13. Heijman E, Aben JP, Penners C, Niessen P, Guillaume R, van Eys G, Nicolay K, Strijkers GJ . Evaluation of manual and automatic segmentation of the mouse heart from CINE MR images. J Magn Reson Imaging 27:86-93, 2008.

14. Hiller KH, Waller C, Haase A, Jakob PM . Magnetic resonance of mouse models of cardiac disease. Handb Exp Pharmacol: 245-257, 2008.

15. Iltis I, Kober F, Dalmasso C, Cozzone PJ, Bernard M. Noninvasive characterization of myocardial blood flow in diabetic, hypertensive, and diabetic-hypertensive rats using spin-labeling MRI. Microcirculation 12:607-614, 2005.

16. Jakob PM, Griswold MA, Edelman RR, Manning WJ, Sodickson DK. Accelerated cardiac imaging using the SMASH technique. J Cardiovasc Magn Reson 1: 153-157, 1999.

17. Kenis H, Zandbergen HR, Hofstra L, Petrov AD, Dumont EA, Blankenberg FD, Haider N, Bitsch N, Gijbels M, Verjans JW, Narula N, Narula J, Reutelingsperger CP. Annexin A5 uptake in ischemic myocardium: demonstration of reversible phosphatidylserine externalization and feasibility of radionuclide imaging. J Nucl Med 51: 259-267, 2010.

18. Oostendorp M, Douma K, Wagenaar A, Slenter JM, Hackeng TM, van Zandvoort MA, Post MJ, BackesWH . Molecular magnetic resonance imaging of myocardial angiogenesis after acute myocardial infarction.Circulation 121: 775-783, 2010. 
19. Palojoki E, Saraste A, Eriksson A, Pulkki K, Kallajoki M, Voipio-Pulkki LM, Tikkanen I . Cardiomyocyte apoptosis and ventricular remodeling after myocardial infarction in rats. Am J Physiol Heart Circ Physiol 280:H2726-H2731, 2001.

20. Pruessmann KP, Weiger M, Scheidegger MB, Boesiger P. SENSE: sensitivity encoding for fast MRI.Magn Reson Med 42: 952-962, 1999.

21. Roemer PB, Edelstein WA, Hayes CE, Souza SP, Mueller OM . The NMR phased array. Magn Reson Med 16: 192-225, 1990.

22. Ross AJ, Yang Z, Berr SS, Gilson WD, Petersen WC, Oshinski JN, French BA. Serial MRI evaluation of cardiac structure and function in mice after reperfused myocardial infarction. Magn Reson Med 47: 1158-1168,2002.

23. Ruff J, Wiesmann F, Hiller KH, Voll S, von Kienlin M, Bauer WR, Rommel E, Neubauer S, Haase A Magnetic resonance microimaging for noninvasive quantification of myocardial function and mass in the mouse. Magn Reson Med 40: 43-48, 1998.

24. Russell JC, Proctor SD. Small animal models of cardiovascular disease: tools for the study of the roles of metabolic syndrome, dyslipidemia, and atherosclerosis. Cardiovasc Pathol 15: 318-330, 2006.

25. Schneider JE, Cassidy PJ, Lygate C, Tyler DJ, Wiesmann F, Grieve SM, Hulbert K, Clarke K, NeubauerS . Fast, high-resolution in vivo cine magnetic resonance imaging in normal and failing mouse hearts on a vertical 11.7 T system. J Magn Reson Imaging 18: 691-701, 2003.

26. Shohet RV, Kisanuki YY, Zhao XS, Siddiquee Z, Franco F, Yanagisawa M . Mice with cardiomyocyte-specific disruption of the endothelin-1 gene are resistant to hyperthyroid cardiac hypertrophy. Proc Natl Acad Sci USA 101: 2088-2093, 2004.

27. Sodickson DK. Tailored SMASH image reconstructions for robust in vivo parallel MR imaging. Magn Reson Med 44: 243-251, 2000.

28. Uemura K, Pisa Z . Recent trends in cardiovascular disease mortality in 27 industrialized countries. World Health Stat Q 38: 142-162, 1985.

29. Uemura K, Pisa Z . Trends in cardiovascular disease mortality in industrialized countries since 1950.World Health Stat Q 41: 155-178, 1988.

30. Wiesmann F, Frydrychowicz A, Rautenberg J, Illinger R, Rommel E, Haase A, Neubauer S. Analysis of right ventricular function in healthy mice and a murine model of heart failure by in vivo MRI. Am J Physiol Heart Circ Physiol 283: H1065-H1071, 2002.

31. Young AA, Barnes H, Davison D, Neubauer S, Schneider JE . Fast left ventricular mass and volume assessment in mice with three-dimensional guide-point modeling. J Magn Reson Imaging 30: 514-520, 2009. 



\section{CHAPTER 6}

\section{Annexin A5 uptake in ischemic}

\section{myocardium: Demonstration of reversible}

phosphatidylserine externalization and

\section{feasibility of radionuclide imaging}

H. Kenis, H.R. Zandbergen, L. Hofstra, A.D. Petrov, E. A. Dumont, F.D. Blankenberg,

N. Haider, N. Bitsch, M. Gijbels, J.W.H. Verjans, N. Narula, J. Narula and C.P.M. Reutelingsperger 


\section{Abstract}

Ischemic insult to the myocardium is associated with cardiomyocyte apoptosis. Because apoptotic cell death is characterized by phosphatidylserine externalization on cell membrane and annexin-A5 (AA5) avidly binds to phosphatidylserine, we hypothesized that radiolabeled AA5 should be able to identify the regions of myocardial ischemia.

Methods: Models of brief myocardial ischemia by the occlusion of the coronary artery for $10 \mathrm{~min}$ (I-10) and reperfusion for $180 \mathrm{~min}$ (R-180) for the detection of phosphatidylserine exteriorization using ${ }^{99 \mathrm{~m}} \mathrm{Tc}$-labeled AA5 and $\gamma$-imaging were produced in rabbits. ${ }^{99 \mathrm{~m}}$ Tc-AA5 uptake after brief ischemia was compared with an I-40/R-180 infarct model. Histologic characterization of both myocardial necrosis and apoptosis was performed in ischemia and infarct models. Phosphatidylserine exteriorization was also studied in a mouse model, and the dynamics and kinetics of phosphatidylserine exposure were assessed using unlabeled recombinant AA5 and AA5 labeled with biotin, Oregon Green, or Alexa 568. Appropriate controls were established.

Results: Phosphatidylserine exposure after ischemia in the rabbit heart could be detected by radionuclide imaging with ${ }^{99 \mathrm{~m}} \mathrm{Tc}-\mathrm{AA} 5$. Pathologic characterization of the explanted rabbit hearts did not show apoptosis or necrosis. Homogenization and ultracentrifugation of the ischemic myocardial tissue from rabbit hearts recovered two thirds of the radiolabeled AA5 from the cytoplasmic compartment. Murine experiments demonstrated that the cardiomyocytes expressed phosphatidylserine on their cell surface after an ischemic insult of 5 min. Phosphatidylserine exposure occurred continuously for at least $6 \mathrm{~h}$ after solitary ischemic insult. AA5 targeted the exposed phosphatidylserine on cardiomyocytes; AA5 was internalized into cytoplasmic vesicles within 10-30 min. Twenty-four hours after ischemia, cardiomyocytes with internalized AA5 had restored phosphatidylserine asymmetry of the sarcolemma, and no detectable phosphatidylserine remained on the cell surface. The preadministration of a pan-caspase inhibitor, zVAD-fmk, prevented phosphatidylserine exposure after ischemia.

Conclusions: After a single episode of ischemia, cardiomyocytes express phosphatidylserine, which is amenable to targeting by AA5, for at least $6 \mathrm{~h}$. Phosphatidylserine exposure is transient and internalized in cytoplasmic vesicles after AA5 binding, indicating the reversibility of the apoptotic process.

\section{Keywords}

programmed cell death, radionuclide imaging, caspase, apoptosis, ischemic memory 
Although it has been traditionally believed that most heart muscle cells during the ischemic insult to the myocardium are lost by necrosis, it is now being increasingly recognized that apoptosis plays an important role in the cell death. Apoptosis may either occur concurrently or precede the process of necrotic cell death (1). Unlike necrosis, apoptosis is associated with a genetically programmed cascade of intracellular events and may be amenable to intervention. Spontaneous recovery from an active cell death program has been reported in the nematode Caenorhabditis elegans, and the recovery may occur even after the shrinkage of the cells and activation of CED-3, a caspase-3 homolog (2). Although no experimental evidence is available, a similar recovery has been hypothesized in mammalian cell systems (3), and the use of caspase inhibitors in myocardial ischemia has resulted in the substantial salvage of cardiomyocyte death. The use of caspase inhibitors is associated with the reduction in the extent of both apoptosis and necrosis and suggests that these 2 components of cell death are interrelated (4). Thus, the feasibility of the noninvasive detection of apoptosis should help maximize the potential of salvage.

Caspase-3 activation during mammalian cell apoptosis is associated with phosphatidylserine expression on apoptotic cells (5). Annexin-A5 (AA5), which has a nanomolar affinity for binding to phosphatidylserine, has been used for the in vitro $(6,7)$ and in vivo $(8,9)$ detection of apoptosis. Radiolabeled AA5 has been successfully used, both clinically and experimentally, for the localization of apoptosis in the myocardial infarcts. This study evaluated whether apoptosis was initiated in brief episodes of myocardial ischemia and whether AA5 would be useful for the detection of ischemia. For this purpose, we performed molecular imaging with ${ }^{99 \mathrm{~m}}$ Tc-labeled AA5 in an experimental rabbit model of brief coronary occlusion. Because myocardial ischemia is a brief event, it was important to define the time window after the initial ischemic insult for the possible detection of apoptosis by imaging. As such, for the characterization of the length and duration of phosphatidylserine exposure we used AA5 labeled with biotin or fluorophores in mice after brief occlusion of a coronary artery.

\section{Materials and methods}

\section{Preparation of AA5 probes for in vivo use}

Human AA5 was produced by expression in Escherichia coli (10). The recombinant AA5 retained its phosphatidylserine binding activity equivalent to the native AA5. In addition to the AA5, 2 mutants were used for the control experiments, one that had lost its phosphatidylserine binding capacity and another that did not internalize after surface phosphatidylserine binding. The cDNA of AA5 mutant M1234 (E72D, D144N, E228A, and D303N) (a kind gift by Dr. Françoise Russo-Marie) has 4 defective calcium binding sites (11). The AA5 mutant M23 was generated by subcloning the mutations D144N and 
E228A into the AA5 CDNA. The recombinant AA5 and the mutants M23 and M1234 were labeled with either Oregon Green or Alexa 568-succinimidylester according to the manufacturer's protocol (Molecular Probes). The labeled proteins, with 1:1 stoichiometry, were purified from the mixtures by MonoQ chromatography with Acta Explorer (Amersham-Pharmacia). In addition, hydrazinonicotinamide-derivatized AA5 was prepared as previously described, without affecting phosphatidylserine binding activity, for radiolabeling with ${ }^{99 \mathrm{~m}} \mathrm{Tc}$ (12). The radiolabeled material retained specific activity, ranging from 3,700 to $7,400 \mathrm{kBq}(100$ to $200 \mu \mathrm{Ci}$ ) of protein per microgram. The AA5 tracers used in this study are shown in Table 1, and protocols are shown in Table 2.

TABLE 1. Recombinant AA5 Tracers

\begin{tabular}{lccll}
\hline AA5 type & $\begin{array}{c}\text { Phosphatidylserine } \\
\text { binding capability }\end{array}$ & $\begin{array}{c}\text { Internalization } \\
\text { capability }\end{array}$ & Label & Dose \\
\hline AA5 & + & + & Biotin & $25 \mathrm{mg} / \mathrm{kg}$ \\
AA5 & + & + & Alexa 568 & $2.5 \mathrm{mg} / \mathrm{kg}$ \\
AA5 & + & + & Oregon Green & $2.5 \mathrm{mg} / \mathrm{kg}$ \\
AA5 M23 & + & - & Alexa 568 & $2.5 \mathrm{mg} / \mathrm{kg}$ \\
AA5 M1234 & - & - & FITC & $2.5 \mathrm{mg} / \mathrm{kg}$ \\
AA5 Hynic & + & + & $99 \mathrm{~m} \mathrm{Tc}$ & $30-40 \mu \mathrm{mg} / \mathrm{kg}$ \\
\hline
\end{tabular}

TABLE 2. Experimental Protocols and Conclusions

\begin{tabular}{|c|c|c|c|c|c|}
\hline $\begin{array}{l}\text { Exposure } \\
\text { [model] }\end{array}$ & $\begin{array}{l}\text { AA5 } \\
\text { and tracer }\end{array}$ & $\begin{array}{l}\text { Time of } \\
\text { injection }\end{array}$ & $\begin{array}{l}\text { Ischemia } \\
\text { (min) }\end{array}$ & $\begin{array}{l}\text { Reperfusion } \\
\text { (min) }\end{array}$ & Conclusion \\
\hline \multicolumn{6}{|c|}{${ }^{99 m}$ Tc-AA5-based imaging of externalized PS in ischemic myocardium [rabbit] } \\
\hline 1 & ${ }^{99 \mathrm{~m}} \mathrm{Tc}-\mathrm{AA} 5$ & At reperfusion & 10 & $180 \mathrm{~min}$ & $\begin{array}{l}\text { Brief ischemia results in detectable PS } \\
\text { exposure but no apoptosis or necrosis } \\
\text { seen histopathologically }\end{array}$ \\
\hline 2 & ${ }^{99 m}$ Tc-AA5 & At reperfusion & 40 & $180 \mathrm{~min}$ & $\begin{array}{l}\text { Prolonged ischemia results in } \\
\text { detectable PS exposure, apoptosis, } \\
\text { and necrosis }\end{array}$ \\
\hline
\end{tabular}

\begin{tabular}{lllll}
\hline \multicolumn{2}{l}{ Cardiomyocytes expose PS after brief ischemia [mouse] } \\
1 & BA5 & 5 & $90 \mathrm{~min}, 24 \mathrm{~h}$ & $\begin{array}{l}\text { Brief ischemia results in PS exposure } \\
\text { but no TUNEL positivity }\end{array}$ \\
2 & AA5 & Before ischemia & $30 \quad 90 \mathrm{~min}, 24 \mathrm{~h}$ & $\begin{array}{l}\text { Prolonged ischemia results in PS } \\
\text { exposure and TUNEL positivity }\end{array}$ \\
\hline
\end{tabular}

PS and AA5 are internalized in cardiomyocytes [mouse]

1 AA5 Oregon At reperfusion $\quad 5 \quad 90 \mathrm{~min} \quad$ Brief ischemia results in PS exposure Green on cardiomyocytes

AA5 Alexa 30 min before that bind and internalize AA5

568 sacrifice 


\begin{tabular}{|c|c|c|c|c|c|}
\hline $\begin{array}{l}\text { Exposure } \\
\text { [model] }\end{array}$ & $\begin{array}{l}\text { AA5 } \\
\text { and tracer }\end{array}$ & $\begin{array}{l}\text { Time of } \\
\text { injection }\end{array}$ & $\begin{array}{l}\text { Ischemia } \\
\text { (min) }\end{array}$ & $\begin{array}{l}\text { Reperfusion } \\
\text { (min) }\end{array}$ & Conclusion \\
\hline \multicolumn{6}{|c|}{ Characterization of PS-dependent internalization [mouse] } \\
\hline 1 & $\begin{array}{l}\text { AA5 M23- } \\
\text { Alexa } 568\end{array}$ & Before ischemia & 5 & $90 \mathrm{~min}$ & $\begin{array}{l}\text { 2-dimensional crystallization of AA5 is } \\
\text { mandatory for internalization }\end{array}$ \\
\hline 2 & $\begin{array}{l}\text { AA5 M1234- } \\
\text { FITC }\end{array}$ & - Before ischemia & 5 & $90 \mathrm{~min}$ & $\begin{array}{l}\text { PS binding of AA5 is mandatory for } \\
\text { internalization }\end{array}$ \\
\hline \multicolumn{6}{|c|}{ Cardiomyocytes expose PS up to $6 \mathrm{~h}$ after brief ischemia [mouse] } \\
\hline 1 & AA5-biotin & $\begin{array}{l}10 \text { min before } \\
\text { sacrifice }\end{array}$ & 5 & $\begin{array}{l}90 \mathrm{~min}, 3 \mathrm{~h}, 6 \mathrm{~h} \text {, } \\
24 \mathrm{~h}\end{array}$ & $\begin{array}{l}\text { Brief ischemia results in caspase- } \\
\text { dependent PS exposure }\end{array}$ \\
\hline \multicolumn{6}{|c|}{ PS exposure is a transient process [mouse] } \\
\hline \multirow[t]{2}{*}{1} & $\begin{array}{l}\text { AA5 Oregon } \\
\text { Green }\end{array}$ & At reperfusion & 5 & $90 \mathrm{~min}, 6 \mathrm{~h}, 24 \mathrm{~h}$ & $\begin{array}{l}\text { Brief ischemia results in PS exposure } \\
\text { that is transient and persists up to } 6 \mathrm{~h}\end{array}$ \\
\hline & $\begin{array}{l}\text { AA5 Alexa } \\
568\end{array}$ & $\begin{array}{l}10 \text { min before } \\
\text { sacrifice }\end{array}$ & & & \\
\hline
\end{tabular}

PS = phosphatidylserine.

M23 and M1234 are AA5 mutants.

\section{Surgical induction of brief ischemia in rabbits and mice}

Myocardial ischemia and reperfusion models were produced in rabbits and mice as reported previously $(13,14)$. In the New Zealand White rabbits, the heart was exposed by mid sternotomy. Brief myocardial ischemia was produced by ligation of the lateral branch of the left coronary artery for $10 \mathrm{~min}$ (I-10), followed by reperfusion for $180 \mathrm{~min}$ (R-180). The myocardial infarction model was created for comparison to brief ischemia by a 40-min occlusion of the coronary artery, followed by reperfusion for $180 \mathrm{~min}$ (I40/R-180) (positive control). In addition, sham-operated animals were used as negative controls for imaging experiments.

In the mouse model, the heart was exposed by left thoracotomy. The left coronary artery was ligated for $5 \mathrm{~min}$ (I-5) and reperfusion was established for $90 \mathrm{~min}$ (R-90), $3 \mathrm{~h}$ (R-180), 6 h (R-360), or 24 h (R-24 h) (Table 2). Ischemia and reperfusion were performed once only for all experiments, and no preconditioning or recurrent ischemia experiments were undertaken. For positive control, infarction was produced by coronary arterial occlusion for 30 min with reperfusion times of $90 \mathrm{~min}$ and $24 \mathrm{~h}$ (I-30/R-90 or I30/R-24 h). In a subset of animals, pan-caspase inhibitor zVAD-fmk ( $3 \mathrm{mg} / \mathrm{kg}$; BIOMOL) was injected through an intravenous line into the jugular vein $10 \mathrm{~min}$ before ischemia to assess whether the cascade of apoptosis could be aborted.

Imaging of Rabbit Myocardial Ischemia with ${ }^{99 \mathrm{~m}}$ Tc-AA5 and Pathologic Characterization

To investigate the potential of imaging myocardial ischemia, ${ }^{99 \mathrm{~m}}$ Tc-labeled AA5 was intravenously administered to the rabbits subjected to brief myocardial ischemia (I-10/R$180 ; n=6)$. Concurrent studies were performed in the prolonged ischemia (I/R-40/180; $n$ 
= 6) model for positive controls and sham-operated (I-0/R-0; $n=4)$ hearts for negative controls. ${ }^{99 \mathrm{~m}} \mathrm{Tc}-\mathrm{AA} 5\left(30-40 \mu \mathrm{g}\right.$ of AA5/kg; $444 \pm 37 \mathrm{MBq}$ of $\left.{ }^{99 \mathrm{~m}} \mathrm{Tc}[12 \pm 1 \mathrm{mCi}]\right)$ was administered at the time of reperfusion through the marginal vein of the ear. Animals were killed after $3 \mathrm{~h}$ with an overdose of pentobarbital, and explanted hearts were subjected to ex vivo planar imaging (X-SPECT; Gamma Medica, Inc.) for $15 \mathrm{~min}$ in a $128 \times 128 \mathrm{ma}$ trix using a 1-mm pinhole collimator. Quantitative uptake of ${ }^{99 \mathrm{~m}}$ Tc-AA5 was determined by well-type $\gamma$-counter (PerkinElmerWallac Inc.) and reflected as percentage injected dose per gram (\%ID/g). Tissue from the ischemic zone and remote myocardium were subjected to ultracentrifugal isolation of the cell membrane, cytoplasm, and other subcellular organelles to localize the ${ }^{99 \mathrm{~m}}$ Tc-AA5 uptake, based on the methodology described previously (14). In addition to subcellular analysis, myocardial tissue sections from the ischemic (or infarcted) and remote myocardial regions were stained by hematoxylin and eosin for standard histology. Tissue sections were also processed for the identification of apoptosis by terminal deoxynucleotidyl transferase-mediated dUTP nick-end labeling (TUNEL) (in situ cell death detection kit, POD; Roche) as described previously (15).

Targeting of Phosphatidylserine Exposure in Murine Models of Ischemia and Pathologic Characterization

For the characterization of surface phosphatidylserine exposure in response to ischemia, unlabeled recombinant human AA5 or fluorescent recombinant human AA5 was administered through a line inserted into the jugular vein of mice. The data were collected for 3 mice in each experimental group. To confirm the phosphatidylserine exteriorization, AA5 was used in I-5/R-90 and I-5/R-24 h mouse models. To assess the length of time for which the phosphatidylserine was expressed, I-5/R-90, I-5/R-180, I-5/R-360, I5/R-24 h, I-30/R-90, and I-30/R-24 h models were studied with AA5-biotin injected at the onset of reperfusion. Conversely, to evaluate the fate of expressed phosphatidylserine, AA5-Oregon Green (2.5 mg/kg) and AA5-Alexa $568(2.5 \mathrm{mg} / \mathrm{kg})$ were used at 2 different time points after reperfusion, at the onset of reperfusion and $10 \mathrm{~min}$ before sacrifice. The hearts were removed and snap-frozen in liquid nitrogen for the histologic localization of exogenously administered AA5 and apoptosis. To study the internalization of AA5 occurring in vivo, a mutant of AA5 M23-Alexa 568, which binds to phosphatidylserine but does not induce its internalization, was injected before reperfusion. To test whether the internalization of AA5 was phosphatidylserine-dependent, a mutant of AA5 M1234-Oregon Green, which does not bind to phosphatidylserine, was injected before reperfusion. Both experiments with mutant AA5 were performed in the I-5/R-90 model ( $n=3$ each).

For the localization of the fluorescent AA5, frozen 7- $\mu$ m tissue sections were obtained, dried overnight, and mounted in 4,6-diamino-2-phenylindole-containing medium (Vector Laboratories) and examined by fluorescence microscopy. Intravenously administered human recombinant AA5 was tracked by immunohistochemical staining of frozen tissue sections. The tissue sections were dried overnight and fixed in dry acetone 
for 10 min at room temperature. After the blocking of endogenous peroxidase, sections were stained with an antibody against AA5 (rabbit polyclonal antihuman AA5; Hyphen Biomed). AA5 was detected by polyclonal horseradish peroxidase-labeled goat antirabbit antibody (Powervision peroxidase detection system; Immunovision Technologies). In contrast, biotin-AA5 was tracked by direct avidin/biotin conjugate peroxidase staining without the need for anti-AA5 antibody. Frozen tissue sections were also stained with hematoxylin and eosin for standard histology and stained by TUNEL (in situ cell death detection kit, POD; Roche) for the identification of apoptosis, as described previously (15).

\section{RESULTS}

\section{Radionuclide imaging of brief myocardial ischemia is feasible}

To explore whether phosphatidylserine exposure could be used potentially to detect recent ischemic events, ${ }^{99 \mathrm{~m}} \mathrm{Tc}-\mathrm{AA} 5$ was administered intravenously in the $1-10 / \mathrm{R}-180$ rabbit model at the time of reperfusion, and imaging of the explanted hearts was performed after $3 \mathrm{~h}$ (14). Significant radiolabeled ${ }^{99 \mathrm{~m}} \mathrm{Tc}-\mathrm{AA} 5$ uptake in the zone of initial ischemia was observed (Fig. 1A). ${ }^{99 m}$ Tc-AA5 uptake in the ischemic region was $9 \pm 3$-fold higher than in the normal myocardium $(0.27 \pm 0.16 \% \mathrm{ID} / \mathrm{g}$ vs. $0.03 \pm 0.01 \% \mathrm{ID} / \mathrm{g})$. Further, at least $85 \%$ of the radioactivity was recovered from the ultracentrifugally isolated cytoplasmic and organelle compartment of the cells, suggesting that ${ }^{99 \mathrm{~m}} \mathrm{Tc}$-AA5 bound to phosphatidylserine was internalized. Although enhanced uptake of ${ }^{99 \mathrm{~m}}$ Tc-AA5 had occurred in the ischemic regions, no histologic evidence of apoptosis or necrosis in the hearts was observed (Fig. 1B). Prolonged ischemia and reperfusion (I-40/R-180) was associated with prominent ${ }^{99 \mathrm{~m}} \mathrm{Tc}-\mathrm{AA} 5$ uptake $(0.55 \pm 0.3 \% \mathrm{ID} / \mathrm{g})$ (Fig. 1A), and apoptosis and necrosis (Fig. 1B). The ${ }^{99 m}$ Tc-AA5 uptake in the sham-operated animals $(0.042 \pm$ $0.012 \%(\mathrm{D} / \mathrm{g}$ ) was similar to the uptake in the remote nonischemic myocardium. The data from the rabbit model indicated that the phosphatidylserine exposure occurred after a brief episode of ischemia and may serve as a marker of ischemic memory in the myocardium. Data also suggested that ${ }^{99 \mathrm{~m}} \mathrm{Tc}-\mathrm{AA} 5$ accumulated intracellularly in ischemic cells. These data confirmed our previous in vitro observations (16) that AA5 mediated internalization of phosphatidylserine-positive membrane patches. To characterize the phosphatidylserine exposure observed in the rabbit imaging experiments, multiple sets of mouse experiments were conducted. 

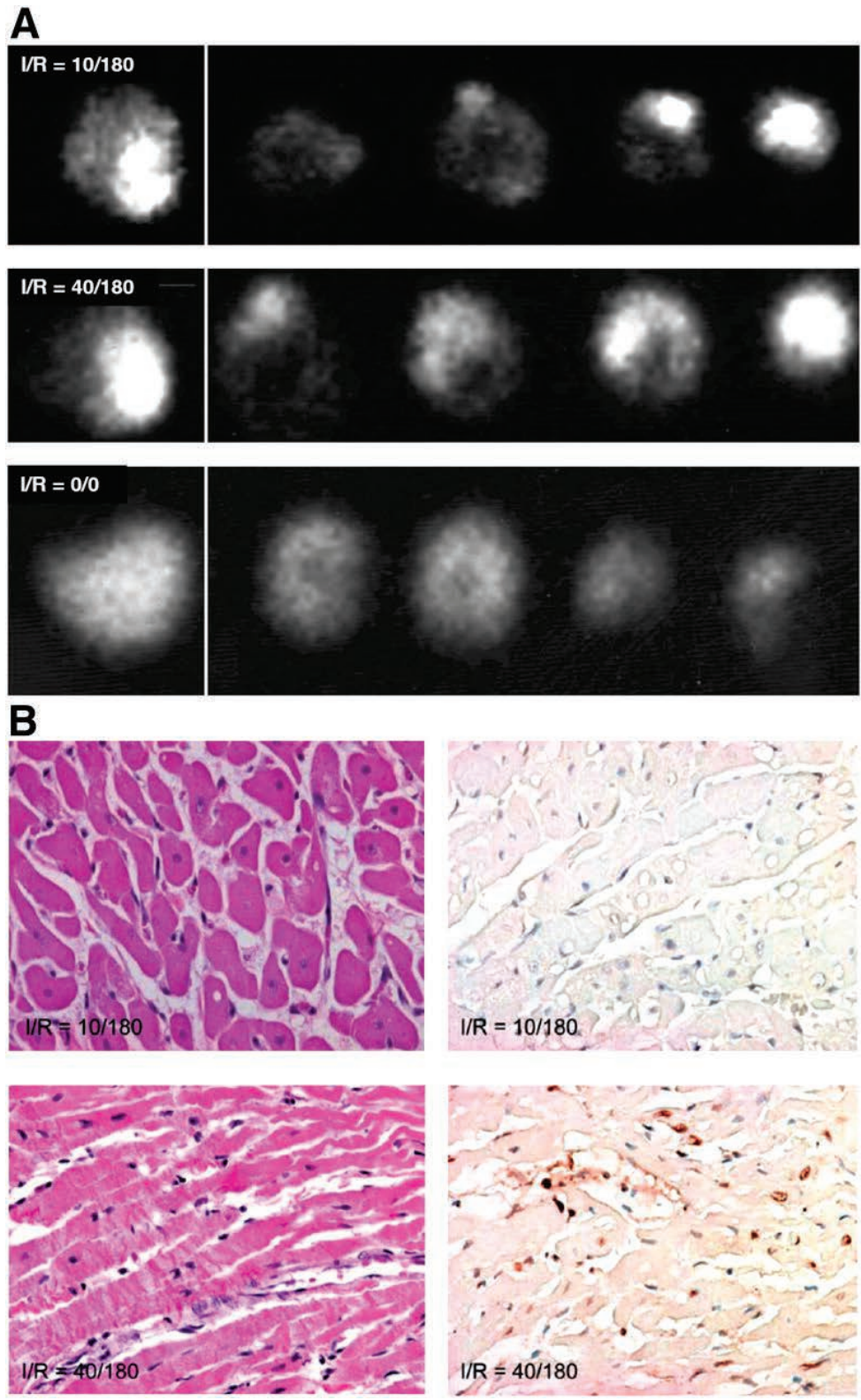

FIGURE 1. ${ }^{99 \mathrm{~m}}$ Tc-AA5-based imaging of externalized phosphatidylserine in ischemic myocardium. (A, top) In I10/R-180 model in rabbit, radiolabeled AA5 clearly delineates ischemic zone with 9-fold higher uptake than in remote myocardium. Uptake is seen in $\gamma$-image of whole heart and slices. ( $B$, top) On tissue sections, no necrosis (left) or apoptosis (right) was observed in ischemic zone. In prolonged ischemic model (I-40/R-180) (A, middle), intense ${ }^{99 \mathrm{~m}} \mathrm{Tc}-\mathrm{AA} 5$ uptake is seen; pathologic characterization reveals ( $\mathrm{B}$, bottom) extensive evidence of necrosis (left) and apoptosis (right). Necrosis is represented by contraction bands and apoptosis by TUNEL staining. No ${ }^{99 \mathrm{~m}}$ Tc-AA5 uptake is seen in sham-operated animals (A, bottom). This experiment provides preliminary data generating hypothesis that hot-spot imaging of ischemia by radiolabeled AA5 is feasible. 


\section{Phosphatidylserine exposure occurs after brief ischemia but not cell death}

To establish the phosphatidylserine exposure characteristics after brief episodes of ischemia observed in the rabbit model, a series of experiments was performed in mice. In the first set of experiments, I-5 hearts received human recombinant AA5 before coronary occlusion, and its localization was traced by immunoperoxidase staining using antihuman AA5 antibody in myocardial sections (13). AA5-positive cardiomyocytes were observed in the ischemic area of the hearts in $1-5$ animals that were subjected to reperfusion for $90 \mathrm{~min}$ and $24 \mathrm{~h}$ (Fig. 2). Cardiomyocytes of the nonischemic area and cardiomyocytes from sham-operated animals did not bind AA5. The AA5 was localized to the intracellular compartment. On TUNEL staining, only rare apoptotic cells were observed in the ischemic area of I-5/R-90 animals (Fig. 2). These findings suggested that the apoptosis program was initiated, phosphatidylserine was exposed that was available for targeting by AA5, and AA5 was internalized. However, because TUNEL-positive cells were not seen even after phosphatidylserine exposure had occurred, the apoptotic process was aborted on reperfusion after brief ischemia. As compared with the I-5/R-90 ischemia model, significantly more myocytes were AA5-positive in I-30/R-90 mice, and many AA5positive cardiomyocytes demonstrated TUNEL-positive nuclei. This set of experiments confirmed the data obtained in the rabbit model that the phosphatidylserine externalization had occurred after brief episodes of ischemia and that AA5 ended up being internalized. 
AA5
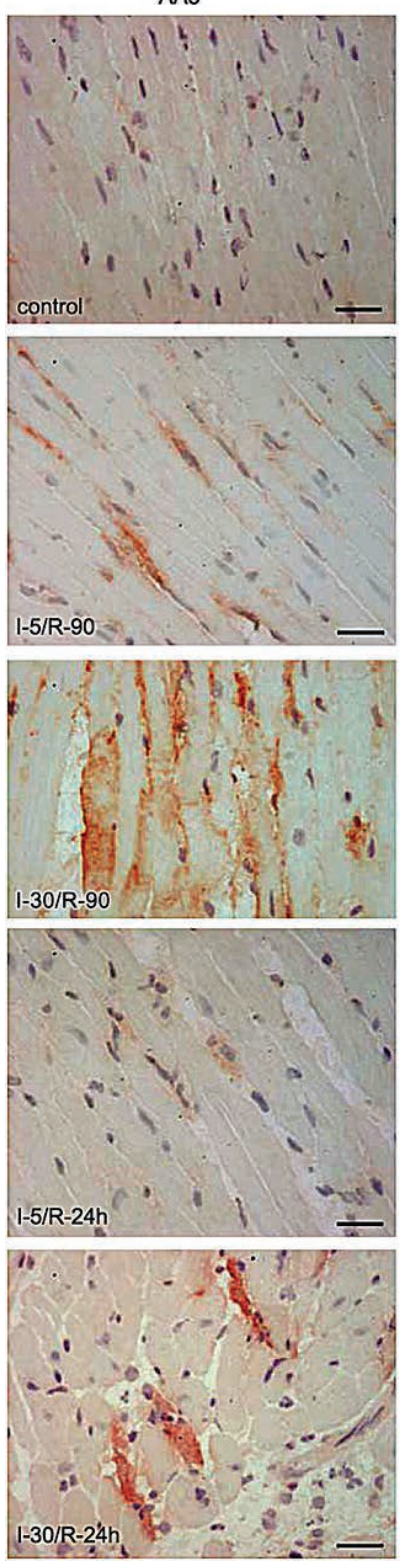

TUNEL
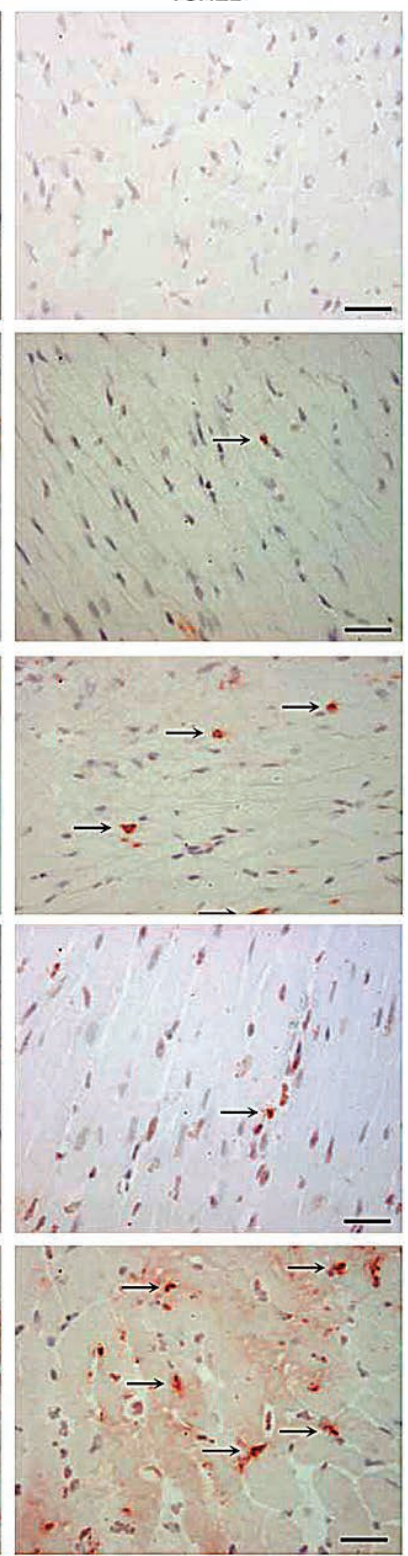

FIGURE 2. Cardiomyocytes expose phosphatidylserine after brief ischemia. Immunostaining for AA5 $(\times 400)$ (left) and apoptosis detection with TUNEL staining ( $\times 400)$ (right) was performed on frozen tissue sections of mouse hearts subjected to different ischemia/reperfusion (I/R) time points. Scale bars represent $25 \mu \mathrm{m}$. AA5 was injected before coronary occlusion in I-5/R-90, I-5/R-24 h, I-30/R-90, and I-30/R-24 h mouse models. Note intracellular location of AA5, more so in prolonged ischemia. Rare apoptotic cells are seen in brief ischemia, unlike abundant apoptotic cells in infarcts. In I-5/R-90 model, preadministration of pan-caspase inhibitor zVADfmk prevents phosphatidylserine exteriorization. Arrows (right panels) refer to TUNEL-positive nuclei. 
Apoptosis assays in vitro have shown that phosphatidylserine exposure is downstream of critical events in the cell death program such as the release of cytochrome c from mitochondria and the activation of caspase-3 $(17,18)$. To evaluate the relationship between brief ischemia and caspase activation, immunoblotting of caspase-3 was undertaken in I5/R-90 hearts. The presence of 12- and 17-kDa split active fragments of caspase-3 indicated the activation of caspase-3; densitometric analysis confirmed a 4-fold increase in activation of caspase-3 (data not shown). Increased densitometric intensity of activated caspase-3 was seen after longer ischemia.

Phosphatidylserine Is Expressed After Brief Ischemia and Internalized After AA5 Binding

From the rabbit heart ultracentrifugation data and the first sets of mouse experiments, it was presumed that AA5 after targeting of the phosphatidylserine was internalized by the ischemic cells. To evaluate the kinetics of the internalization process in vivo after ischemia or reperfusion injury, we performed the second set of experiments. For this purpose, we injected AA5-Oregon Green at the beginning of reperfusion in the 15/R-90 model. As expected, the green AA5 was localized intracellularly. In two separate groups of these I-5/R-90 animals that had already received AA5-Oregon Green at the start of reperfusion, AA5-Alexa 568 was injected either 10 or 30 min before the end of the reperfusion (or sacrificing the animal). AA5-Alexa 568 injected 10 min before sacrifice showed binding almost exclusively to the cardiomyocyte membrane; green AA5 was entirely intracellular (Fig. 3A). However, the injection of AA5-Alexa 56830 min before sacrifice showed colocalization of AA5-Alexa 568 with AA5-Oregon Green within the cytoplasmic compartment of the cell (Fig. 3B), indicating that after AA5 targeting of the phosphatidylserine on the cell surface, the phosphatidylserine-AA5 complex was internalized between 10 and $30 \mathrm{~min}$. These in vivo data confirmed our previously reported in vitro data that AA5 mediates the internalization of phosphatidylserine-positive membrane patches as endocytic vesicles, which detach from the plasma membrane and follow cytoskeleton-dependent trafficking into the cytosol (16). 


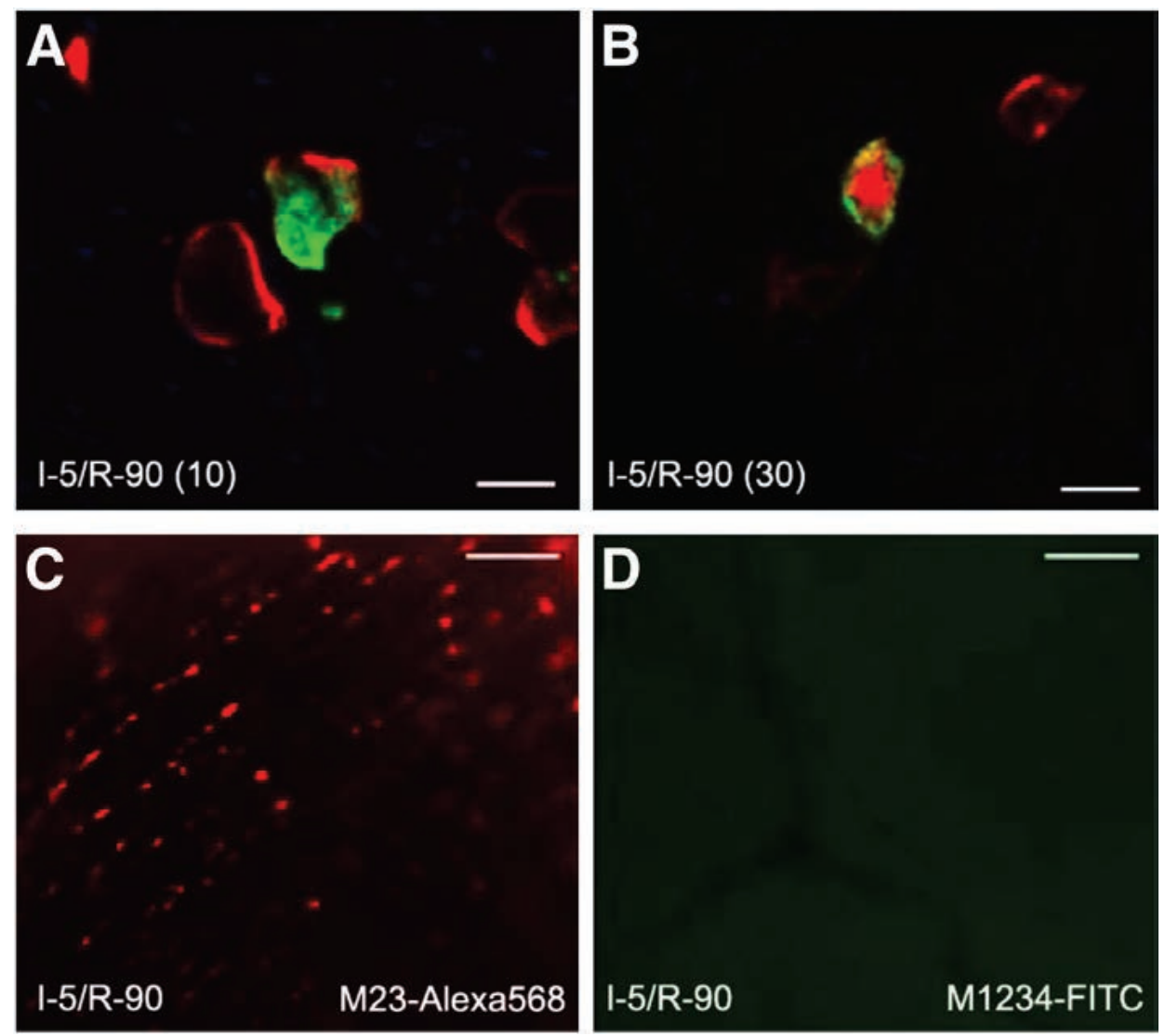

FIGURE 3. Phosphatidylserine and AA5 are internalized in cardiomyocytes. (A) Tissue section $(\times 400)$ of mouse hearts injected with AA5-Oregon Green at beginning of reperfusion and AA5-Alexa 56810 min before end of reperfusion episode in I-5/R-90 model show surface binding and internalization of AA5-Oregon Green. In contrast, only surface binding but no internalization of AA5-Alexa 568 is seen. Parenthesis entry of 10 following I-5/R-90 denotes time of AA5-Alexa 568 administration before the end of R-90 reperfusion period. (B) Injection of AA5-Alexa 56830 min before completion of reperfusion showed colocalization of AA5-Alexa 568 with AA5-Oregon Green within cytoplasmic compartment of cell. Scale bars represent $25 \mu \mathrm{m}$. (C) Ex vivo image $(\times 160)$ of heart of mouse injected with M23-Alexa 568, before 5-min ischemia and 90-min reperfusion (I-5/R90), demonstrates binding to cell membrane but no internalization. (D) Ex vivo image $(\times 160)$ of heart of mouse injected with M1234-FITC before I-5/R-90 shows neither binding nor internalization. Scale bars represent 25 $\mu \mathrm{m}$.

\section{AA5 binding to phosphatidy/serine is necessary for phosphatidy/serine internalization}

To demonstrate that the AA5 targets phosphatidylserine and is subsequently internalized, we conducted the third set of experiments with 2 fluorescently labeled mutant species of AA5. The AA5 mutant M23, by prediction from the available structural data (19), binds to phosphatidylserine but lacks the ability to crystallize on the phosphatidyl- 
serine-expressing membrane surface, the step necessary before internalization. $\mathrm{M} 23-$ Alexa 568 was injected before reperfusion in the I-5/R-90 model. The mutant M23 was observed targeting the cell membrane of the cardiomyocytes in the ischemic zone but was not seen intracellularly (Fig. 3C). Furthermore, to test the phosphatidylserine dependence of the internalization process in vivo, we used yet another AA5 mutant M1234 that lacked the ability to bind to phosphatidylserine. M1234-fluorescein isothiocyanate (FITC) was injected before reperfusion in the I-5/R-90 model. The mutant M1234 did not bind to the cardiomyocyte surface, nor was fluorescence observed intracellularly (Fig. 3D).

Continuous Phosphatidylserine Externalization-Internalization Represents Ischemic Memory

The internalization experiments of the second and third sets raised another important possibility. If phosphatidylserine exposure occurred as early as 5 min after ischemia and internalized within $30 \mathrm{~min}$ after AA5 binding, then cell surface binding of AA5 injected at $80 \mathrm{~min}$ of reperfusion (10 min before the end of a 90-min reperfusion) would suggest an ongoing, continuous, and fresh expression of phosphatidylserine after a solitary initial episode of brief ischemia. To evaluate this possibility, a fourth and fifth sets of experiments were performed with AA5 administration at various time points after brief ischemia up to $24 \mathrm{~h}$.

To demonstrate the length of phosphatidylserine exposure after brief ischemic insult, in the fourth set of experiments, I-5 animals were reperfused for 90, 180, and 360 min and 24 h. AA5-biotin was injected 10 min before sacrifice. Phosphatidylserine exposure was detectable up to $6 \mathrm{~h}$ after the ischemic insult (Fig. 4). No AA5 uptake was observed in the area at risk at $24 \mathrm{~h}$ of reperfusion. It seems that as long as apoptotic machinery remains active after the brief ischemic episode, phosphatidylserine continues being exposed and is amenable to removal from the cell surface, if AA5 is available. This concept was further substantiated by the pretreatment of animals with zVAD-fmk before the induction of brief ischemia (Fig. 4). In the pretreated I-5/R-90 mice, no AA5 binding was seen in these animals, suggesting that the inhibition of caspase successfully prevented phosphatidylserine externalization in response to brief ischemic insult. All experiments indicate that cardiomyocytes recovered and phosphatidylserine exposure resolved if reperfusion was restored in a timely manner or if caspase inhibition was applied. 

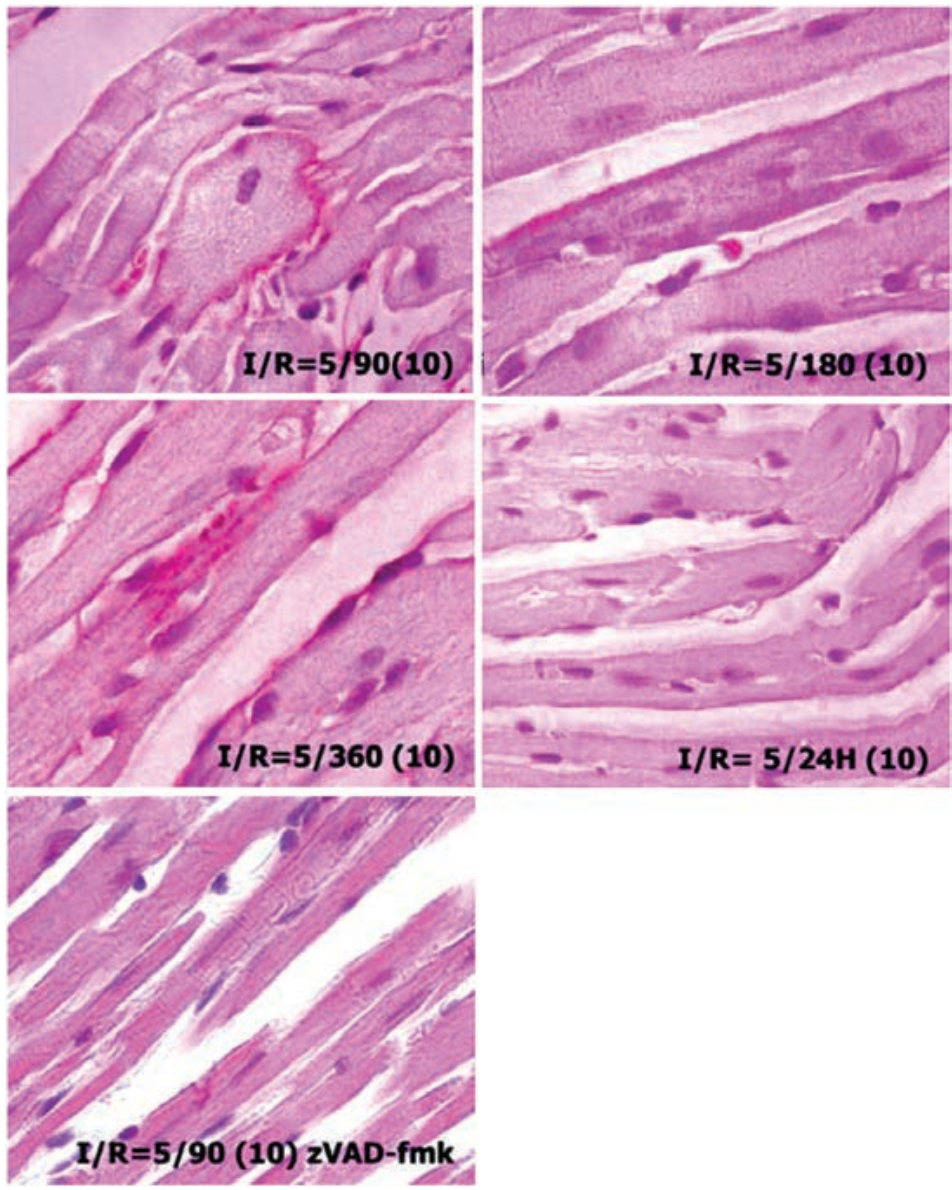

FIGURE 4. Cardiomyocytes expose phosphatidylserine up to $6 \mathrm{~h}$ after brief ischemia. Mouse hearts subjected to $5 \mathrm{~min}$ of ischemia and 90, 180, and $360 \mathrm{~min}$ and $24 \mathrm{~h}$ of reperfusion received $A A 5-$ biotin injected $10 \mathrm{~min}$ before sacrifice (parentheses entry). Cardiomyocytes in ischemic zone demonstrate AA5 binding up to $6 \mathrm{~h}$; cardiomyocytes in I-5/R-24 h do not bind AA5-biotin ( $25 \mathrm{mg} / \mathrm{kg}$ ) when injected $10 \mathrm{~min}$ before sacrifice. zVAD (3 mg/kg) was injected intravenously before application of ischemia in l-5/R-90 model.

To further demonstrate whether phosphatidylserine exposure was a continuous process and the fresh phosphatidylserine was available until $6 \mathrm{~h}$ after the initial brief ischemic episode, we again subjected I-5 mice to R-90, R-360, and R-24 h reperfusion (Fig. 5). AA5-Oregon Green was administered at the onset of reperfusion; green fluorescence was exclusively localized intracellularly. The administration of AA5-Alexa 56810 min before sacrifice revealed that $90 \mathrm{~min}$ and $6 \mathrm{~h}$ after the brief ischemic insult cardiomyocytes with internalized AA5-Oregon Green still bound AA5-Alexa 568 on their cell surface. When AA5-Alexa 568 was injected $24 \mathrm{~h}$ after brief ischemia, the cardiomyocytes with internalized AA5-Oregon Green did not bind AA5-Alexa 568, indicating that these 
cardiomyocytes had already restored phosphatidylserine asymmetry. Although enhanced uptake of AA5 had occurred in the ischemic regions, no histologic evidence of apoptosis or necrosis in the hearts was observed. The data suggested that phosphatidylserine exposure occurred continuously at least up to $6 \mathrm{~h}$ after the ischemic episode and was amenable to AA5 targeting. These sets of experiments further highlighted the possibility of phosphatidylserine expression as a sign of ischemic memory.
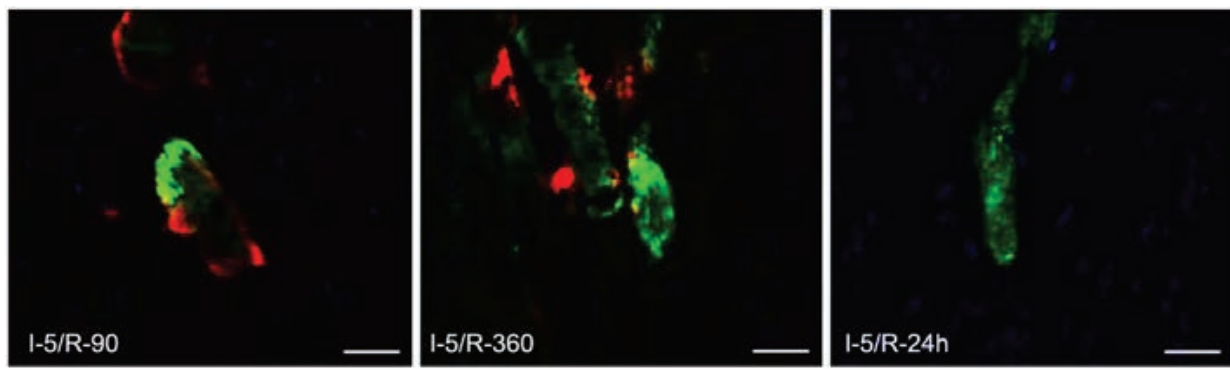

FIGURE 5. Phosphatidylserine exposure and internalization are transient processes. Tissue sections $(\times 400)$ of I-5/R-90, R-360, and R-24 h mouse hearts, injected with AA5-Oregon Green at beginning of reperfusion, followed by injection of AA5-Alexa 56810 min before completion of reperfusion. There is binding of AA5-Alexa 568 to plasma membrane of (internalization) AA5-Oregon Green-positive cells after 90 min and 6 h of reperfusion. After 24 h of reperfusion, AA5-Oregon Green-positive cells do not bind AA5-Alexa 568. This experiment suggests that after brief episode of ischemia, phosphatidylserine exposure, although transient, is continuously exposed for $6 \mathrm{~h}$. Scale bars represent $25 \mu \mathrm{m}$.

\section{Discussion}

Our data show that brief episodes of ischemia result in phosphatidylserine exposure that normally lasts for at least $6 \mathrm{~h}$ after the ischemic insult and offers a target for ${ }^{99 \mathrm{~m}} \mathrm{Tc}$-AA5 for after-the-fact imaging of ischemia (ischemic memory). Phosphatidylserine exposure occurs continuously for the duration because the AA5-phosphatidylserine complex is continuously removed from the cell surface by internalization and new phosphatidylserine is exteriorized. Because the AA5-phosphatidylserine complex is internalized, the signal stays in situ after ${ }^{99 \mathrm{~m}} \mathrm{Tc}-\mathrm{AA} 5$ administration and may concentrate radiolabeled AA5 in the ischemic cells. This explains the possibility of imaging even if phosphatidylserine exposure may not be extensive or intense after brief ischemia. These data deliver another important message. Because phosphatidylserine exposure and caspase activation are the hallmarks of apoptosis (20), it appears that ischemia-induced apoptosis in cardiomyocytes is interrupted by reperfusion such that the cardiomyocytes survive. An escape from activated apoptosis has been reported previously in Caenorhabditis elegans $(2,3)$. During developmental apoptosis, engulfment genes act in concert with CED-3 in cells destined to die in the anterior pharynx. In the absence of activation of the engulfment machinery, such cells show all the features of apoptosis, but cell death does not occur; 
these cells recover completely and remain viable thereafter. Our data confirm recovery from apoptosis in mammalian cells in vivo, despite activation of one of the key executioner caspases, caspase-3. Reversible phosphatidylserine exposure not associated with the execution of apoptosis has been observed in vitro with myocytic cell lines $(21,22)$. Other examples include the activation of B-cell lymphoma cells, which in response to cross-linking of the membrane immunoglobulin receptor stained positive for AA5 but were able to restore their phospholipid asymmetry and resume growth on withdrawal of the stimulus (23). It seems that the phenomenon of recovery from apoptosis may be evolutionarily conserved. However, in contrast to $C$. elegans the absence of engulfment gene activity may not be critical in the recovery of cell death in the heart, because phagocytes enter the ischemic zone only after $24 \mathrm{~h}$. An interesting aspect of the binding of AA5 to externalized phosphatidylserine is that this may offer the cells protection from being recognized by "professional" phagocytes, because externalized phosphatidylserine is necessary for the engulfment of apoptotic cells and serves as an "eat me" signal (20). Clinical studies have shown that plasma AA5 levels are elevated in patients with acute myocardial infarction (24). These data suggest that AA5 may serve as an acute-phase reactant, which may save cells that are susceptible to engulfment. It is also justified to hypothesize that exogenously administered AA5 may accelerate the internalization of externalized phosphatidylserine. The data on the internalization of phosphatidylserine in the presence of AA5 indicate that internalization is not the result of the inhibition of scrambling of phosphatidylserine molecules but results from invagination and vesicle formation of the patches of plasma cell membrane that expose phosphatidylserine (16). Together, our data suggest a novel model of phosphatidylserine exposure and internalization wherein phosphatidylserine exposure is continuously driven by caspase activation and phosphatidylserine internalization is mediated by AA5-aided formation of the endocytic vesicle (16). Cytoplasmic uptake of exogenous AA5 has been previously reported in animal studies of apoptotic cell death during embryogenesis (25), tooth development (26), and stroke (27). The investigators in these studies concluded that the intracellular uptake of exogenous AA5 was due to the ingestion of apoptotic vesicles coated with AA5 by neighboring cells. This mechanism in our model seems unlikely given the lack of apoptotic or necrotic cells in the hearts of animals subjected to brief ischemia. Our unpublished in vitro data further indicate that the perinuclear vesicles in an increased hypercalcemic intracellular milieu may prevent cytochrome c release from mitochondria, supporting the antiapoptotic role of AA5 at least in ischemic heart muscle cells. The reversible phosphatidylserine exposure constitutes an attractive target for the noninvasive detection of myocardial ischemia as a hot-spot imaging agent in addition to traditional stress myocardial perfusion imaging. Furthermore, persistence of phosphatidylserine externalization for at least $6 \mathrm{~h}$ after ischemic stress may allow retrospective diagnosis of cardiac chest pain and support the concept of ischemic memory. This concept would be somewhat similar to the metabolic stunning of myocytes after a myocardial ischemic episode, wherein fatty acid use is interrupted in favor of glucose use. These 2 metabolic 
alterations have been well characterized by the absence of ${ }^{123}$ I-BMIPP uptake (28) and enhanced ${ }^{18}$ F-FDG uptake (29) in the ischemic myocardial territories.

\section{Conclusion}

Our data demonstrate that caspase-3 is activated and phosphatidylserine is externalized in mammalian cells in response to brief ischemia of the heart. Cardiomyocytes destined to die recover from the initiation of the cell death program on restoration of blood flow. The transient exposure of phosphatidylserine by cardiomyocytes recovering from apoptosis may be exploited for diagnostic imaging. Further, AA5 binding to phosphatidylserine leads to the internalization of AA5 into vesicles. Although logically protective, the role of AA5-phosphatidylserine internalization remains to be understood.

\section{Acknowledgments}

The study was supported in part by Joseph DiPalma and Isadore Brodsky Research Funds to Dr. Narula; Dutch Heart Foundation grants NHS2000-D35 and 98.195; Dutch Science Foundation (NWO) grants 902-26-184, 014-80-103, and 912-03-013 to Dr. Reutelingsperger; and the Wynand Pon Foundation grant to Dr. Dumont. 


\section{References}

1. Narula J, Strauss HW. Invited commentary: P.S.* I love you-implications of phosphatidyl serine (PS) reversal in acute ischemic syndromes. J Nucl Med.2003;44:397-399.

2. Hoeppner DJ, Hengartner MO, Schnabel R. Engulfment genes cooperate with ced-3 to promote cell death in Caenorhabditis elegans. Nature.2001;412:202-206.

3. Reddien PW, Cameron S, Horvitz HR. Phagocytosis promotes programmed cell death in C. elegans. Nature. 2001;412:198-202.

4. Yaoita H, Ogawa K, Maehara K, Maruyama Y. Attenuation of ischemia/reperfusion injury in rats by a caspase inhibitor. Circulation.1998;97:276-281.

5. Narula J, Haider N, Arbustini E, Chandrashekhar Y. Mechanisms of disease: apoptosis in heart failureseeing hope in death. Nat Clin Pract Cardiovasc Med. 2006;3:681-688.

6. Vermes I, Haanen C, Steffens-Nakken H, Reutelingsperger C. A novel assay for apoptosis: flow cytometric detection of phosphatidylserine expression on early apoptotic cells using fluorescein labelled annexin V.J Immunol Methods. 1995;184:39-51.

7. van Genderen $H$, Kenis $H$, Lux $P$, et al. In vitro measurement of cell death with the annexin A5 affinity assay. Nat Protoc. 2006;1:363-367.

8. Hofstra L, Liem IH, Dumont EA, et al. Visualisation of cell death in vivo in patients with acute myocardial infarction. Lancet. 2000;356:209-212.

9. Narula J, Acio ER, Narula N, et al. Annexin-V imaging for noninvasive detection of cardiac allograft rejection. Nat Med. 2001;7:1347-1352.

10. Maurer-Fogy I, Reutelingsperger CP, Pieters J, Bodo G, Stratowa C, Hauptmann R. Cloning and expression of cDNA for human vascular anticoagulant, a Ca2+-dependent phospholipid-binding protein. Eur J Biochem. 1988;174:585-592.

11. Mira JP, Dubois T, Oudinet JP, Lukowski S, Russo-Marie F, Geny B. Inhibition of cytosolic phospholipase $\mathrm{A} 2$ by annexin $\mathrm{V}$ in differentiated permeabilized HL-60 cells: evidence of crucial importance of domain I type II Ca2+-binding site in the mechanism of inhibition. J Biol Chem.1997;272:10474-10482.

12. Blankenberg FG, Katsikis PD, Tait JF, et al. In vivo detection and imaging of phosphatidylserine expression during programmed cell death. Proc Natl Acad Sci USA. 1998;95:6349-6354.

13. Dumont EA, Hofstra L, van Heerde WL, et al. Cardiomyocyte death induced by myocardial ischemia and reperfusion: measurement with recombinant human annexin- $\mathrm{V}$ in a mouse model. Circulation.2000;102:1564-1568.

14. Narula J, Petrov A, Pak KY, Lister BC, Khaw BA. Very early noninvasive detection of acute experimental nonreperfused myocardial infarction with ${ }^{99 m}$ Tc-labeled glucarate. Circulation. 1997;95:1577-1584.

15. Kolodgie FD, Petrov A, Virmani R, et al. Targeting of apoptotic macrophages and experimental atheroma with radiolabeled annexin $\mathrm{V}$ : a technique with potential for noninvasive imaging of vulnerable plaque.Circulation. 2003;108:3134-3139.

16. Kenis H, van Genderen H, Bennaghmouch A, et al. Cell surface-expressed phosphatidylserine and annexin A5 open a novel portal of cell entry. J Biol Chem. 2004;279:52623-52629.

17. Martin SJ, Reutelingsperger CP, McGahon AJ, et al. Early redistribution of plasma membrane phosphatidylserine is a general feature of apoptosis regardless of the initiating stimulus: inhibition by overexpression of Bcl-2 and Abl. J Exp Med. 1995;182:1545-1556.

18. Goldstein JC, Waterhouse NJ, Juin P, Evan GI, Green DR. The coordinate release of cytochrome c during apoptosis is rapid, complete and kinetically invariant. Nat Cell Biol. 2000;2:156-162.

19. Huber R, Schneider M, Mayr I, Romisch J, Paques EP. The calcium binding sites in human annexin $V$ by crystal structure analysis at 2.0 A resolution: implications for membrane binding and calcium channel activity. FEBS Lett.1990;275:15-21.

20. Savill J, Fadok V. Corpse clearance defines the meaning of cell death.Nature. 2000;407:784-788.

21. van den Eijnde SM, van den Hoff MJ, Reutelingsperger CP, et al. Transient expression of phosphatidylserine at cell-cell contact areas is required for myotube formation. J Cell Sci. 2001;114:3631-3642. 
22. Kaspar P, Dvorak M. Involvement of phosphatidylserine externalization in the down-regulation of c-myb expression in differentiating C2C12 cells.Differentiation. 2008;76:245-252.

23. Hammill AK, Uhr JW, Scheuermann RH. Annexin $V$ staining due to loss of membrane asymmetry can be reversible and precede commitment to apoptotic death. Exp Cell Res. 1999;251:16-21.

24. Kaneko N, Matsuda R, Hosoda S, Kajita T, Ohta Y. Measurement of plasma annexin V by ELISA in the early detection of acute myocardial infarction. Clin Chim Acta. 1996;251:65-80.

25. van den Eijnde SM, Luijsterburg AJ, Boshart L, et al. In situ detection of apoptosis during embryogenesis with annexin V: from whole mount to ultrastructure. Cytometry. 1997;29:313-320.

26. Bronckers AL, Goei SW, Dumont E, et al. In situ detection of apoptosis in dental and periodontal tissues of the adult mouse using annexin-V-biotin. Histochem Cell Biol. 2000;113:293-301.

27. Mari C, Karabiyikoglu M, Goris ML, Tait JF, Yenari MA, Blankenberg FG. Detection of focal hypoxicischemic injury and neuronal stress in a rodent model of unilateral MCA occlusion/reperfusion using radiolabeled annexin V.Eur J Nucl Med Mol Imaging. 2004;31:733-739.

28. Dilsizian V, Bateman TM, Bergmann SR, et al. Metabolic imaging with beta-methyl-p- $\left.{ }^{123} \mathrm{I}\right]$-iodophenylpentadecanoic acid identifies ischemic memory after demand ischemia. Circulation. 2005;112:21692174.

29. He ZX, Shi RF, Wu YJ, et al. Direct imaging of exercise-induced myocardial ischemia with fluorine-18labeled deoxyglucose and Tc-99m-sestamibi in coronary artery disease. Circulation. 2003;108:12081213. 



\section{CHAPTER 7}

\section{Molecular imaging for efficacy of} pharmacologic intervention in myocardial remodeling

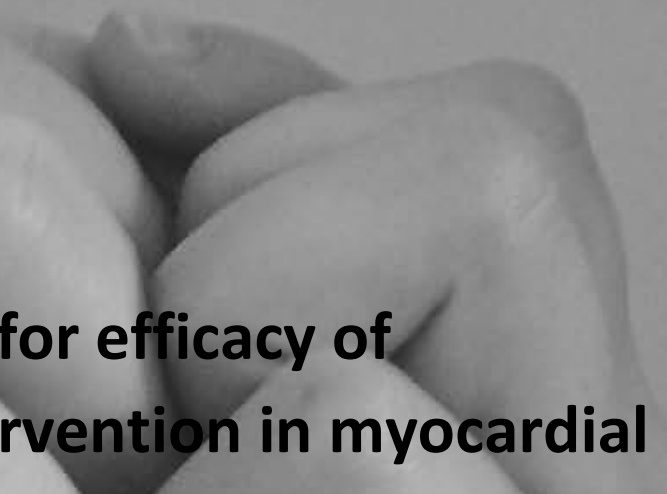

\footnotetext{
S.W.M. van den Borne, S. Isobe, H.R. Zandbergen, P. Li, A.D. Petrov, N.D. Wong,

S. Fujimoto, A. Fujimoto, D. Lovhaug, J.F.M. Smits, M.J.A.P. Daemen, W.M. Blankesteijn,

C. Reutelingsperger, F. Zannad, N. Narula, M.A. Vannan, B. Pitt, L. Hofstra, J. Narula
} 


\section{Abstract}

Objectives. Using molecular imaging techniques, we examined interstitial alterations during postmyocardial infarction (MI) remodeling and assessed the efficacy of antiangiotensin and antimineralocorticoid intervention, alone and in combination.

Background. The antagonists of the renin-angiotensin-aldosterone axis restrict myocardial fibrosis and cardiac remodeling after $\mathrm{MI}$ and contribute to improved survival. Radionuclide imaging with technetium-99m-labeled Cy5.5 RGD imaging peptide (CRIP) targets myofibroblasts and indirectly allows monitoring of the extent of collagen deposition post-MI.

Methods. CRIP was intravenously administered for gamma imaging after 4 weeks of $\mathrm{Ml}$ in 63 Swiss-Webster mice and in 6 unmanipulated mice. Of 63 animals, 50 were treated with captopril $(C)$, losartan $(\mathrm{L})$, spironolactone $(\mathrm{S})$ alone, or in combination $(\mathrm{CL}$, $\mathrm{SC}, \mathrm{SL}$, and SCL), 8 mice received no treatment. Echocardiography was performed for assessment of cardiac remodeling. Hearts were characterized histopathologically for the presence of myofibroblasts and thick and thin collagen fiber deposition.

Results. Acute MI size was similar in all groups. The quantitative CRIP percent injected dose per gram uptake was greatest in the infarct area of untreated control mice $(2.30$ $\pm 0.14 \%)$ and decreased significantly in animals treated with 1 agent $(C$, L, or $S ; 1.71 \pm$ $0.35 \% ; p=0.0002)$. The addition of $2(C L, S C$, or SL $1.31 \pm 0.40 \% ; p<0.0001)$ or 3 agents $(\mathrm{SCL} ; 1.16 \pm 0.26 \% ; \mathrm{p}<0.0001)$ demonstrated further reduction in tracer uptake. The decrease in echocardiographic left ventricular function, strain and rotation parameters, as well as histologically verified deposition of thin collagen fibers, was significantly reduced in treatment groups and correlated with CRIP uptake.

Conclusions. Radiolabeled CRIP allows for the evaluation of the efficacy of neurohumoral antagonists after $\mathrm{MI}$ and reconfirms superiority of combination therapy. If proven clinically, molecular imaging of the myocardial healing process may help plan an optimal treatment for patients susceptible to heart failure.

Key Words. Collagen; remodeling; radionuclide imaging; angiotensin receptors; angiotensin-converting enzyme; mineralocorticoids; heart failure 
Up regulation of the renin-angiotensin-aldosterone axis after myocardial infarction (MI) contributes to the process of cardiac remodeling and hence the evolution of heart failure (1-4). Although this remodeling process is initiated by myocyte loss, interstitial and myocytic alterations continue to occur inexorably even after the initial injury has abated (5-7). Antiangiotensin (8-10) and antialdosterone (11) treatments effectively control this process and delay or prevent the development of heart failure (12-15). These therapeutic interventions reduce the extent of interstitial collagen deposition, which is considered to be the basis of their efficacy ( 1 and 2 ). The combination of these agents has been shown to be more effective than the solitary agents $(16,17)$.

The efficacy of pharmacologic intervention in a clinical milieu is variable, and it is desirable to define optimal use of antiremodeling agents for individual patients. Although pharmacogenomics has been proposed as an important determinant of such a strategy $(18,19)$, the role of phenotypic characterization by novel imaging methods is also being explored (4). It has recently been reported that the extent of new collagen deposition can be assessed by molecular imaging employing technetium-99m-labeled Cy5.5-RGD-imaging peptide (99mTc-CRIP) (20). Through Arg-Gly-Asp (RGD)-based targeting, 99mTc-CRIP binds to the myofibroblasts in the healing infarct and correlates with the extent of new collagen deposition after experimental MI (20). In our preliminary experiment we proposed that such imaging strategy may have a role in identifying the impact of a therapeutic intervention on collagen deposition and hence the efficacy of therapy. In the present study, we evaluated the effect of an angiotensin-converting enzyme inhibitor (ACE-I), an angiotensin receptor blocker (ARB), and a selective aldosterone receptor antagonist (SARA), individually and in combination, on infarct healing by using 99mTc-CRIP imaging.

\section{Methods}

\section{Study protocol}

The present study was performed in 69 Swiss-Webster mice. Of these, MI was produced in 63 mice by occlusion of the left coronary artery. Echocardiograms were obtained before and after coronary occlusion to demonstrate the basal left ventricular (LV) dimensions and function in addition to the infarct size. Of the 63 infarcted mice, 20 mice received a single agent intervention $(1 \mathrm{Rx}) ; 8,6$, and 6 animals were started on spironolactone (S), captopril (C), and losartan (L), respectively. A total of 22 of 63 animals received dual therapy $(2 \mathrm{Rx}) ; 6,8$, and 8 animals were started on a combination of captopril + losartan (CL), spironolactone + captopril (SC), or spironolactone + losartan (SL), respectively, and 8 of 63 post-MI animals received a combination of 3 neurohumoral antagonists (3Rx, spironolactone + captopril + losartan [SCL]) (Table 1). Eight post-MI animals did not receive any pharmacologic agent and served as treatment controls (No Rx), and 5 
post-MI animals received a scrambled version of the imaging peptide (SCRIP) to serve as tracer controls. In addition to 63 infarcted mice, 6 unmanipulated animals were included in the imaging protocol as disease control animals. After 4 weeks, all animals again underwent echocardiographic examination for the characterization of the remodeling process and received 99mTc-labeled CRIP intravenously. After $3.5 \mathrm{~h}$ of CRIP administration, hearts were explanted and ex vivo imaging was performed with a microSPECT camera. The hearts were then sectioned into 3 cross-sectional bread-loaf slices to represent apical (predominantly infarct), middle, and the basal (predominantly remote) areas. All samples were gamma-counted for quantitative 99mTc-CRIP (and SCRIP) uptake. Subsequently, all myocardial specimens were processed for histopathological characterization, particularly the extent of myofibroblast proliferation and thick and thin collagen fiber deposition.

Table 1. Various Treatment Groups, Dosage Schedules, and Quantitative CRIP Uptake

\begin{tabular}{|c|c|c|c|c|c|c|c|c|}
\hline \multirow[t]{2}{*}{ Group } & \multicolumn{3}{|l|}{ Dose } & \multirow[t]{2}{*}{$n$} & \multicolumn{2}{|l|}{ Infarct } & \multicolumn{2}{|l|}{ Remote } \\
\hline & Spironolactone & Captopril & Losartan & & $\%$ ID/g & p Value & $\% \mathrm{ID} / \mathrm{g}$ & $\mathrm{p}$ Value \\
\hline Control & - & - & - & 6 & $0.590 \pm 0.19$ & + & $0.677 \pm 0.162$ & NS \\
\hline No Rx & - & - & - & 8 & $2.302 \pm 0.136$ & $\square$ & $0.921 \pm 0.218$ & NS \\
\hline S & 20 mg/kg/day & - & - & 8 & $1.798 \pm 0.437$ & $\square_{+}$ & $0.903 \pm 0.221$ & NS \\
\hline C & - & 60 mg/kg/day & - & 6 & $1.718 \pm 0.296$ & $\square_{+}$ & $0.867 \pm 0.234$ & NS \\
\hline$L$ & - & - & 20 mg/kg/day & 6 & $1.576 \pm 0.279$ & $\square_{+}$ & $0.752 \pm 0.114$ & NS \\
\hline $\mathrm{CL}$ & - & 30 mg/kg/day & 10 mg/kg/day & 6 & $1.374 \pm 0.549$ & $a_{+}$ & $0.783 \pm 0.272$ & NS \\
\hline SC & 20 mg/kg/day & 60 mg/kg/day & - & 8 & $1.280 \pm 0.408$ & $\square_{+}$ & $0.786 \pm 0.168$ & NS \\
\hline$S L$ & $20 \mathrm{mg} / \mathrm{kg} /$ day & - & 20 mg/kg/day & 8 & $1.293 \pm 0.316$ & $\square_{\dagger}$ & $0.821 \pm 0.364$ & NS \\
\hline $\mathrm{SCL}$ & 20 mg/kg/day & 60 mg/kg/day & 20 mg/kg/day & 8 & $1.157 \pm 0.261$ & $\square_{\dagger}$ & $0.796 \pm 0.196$ & NS \\
\hline
\end{tabular}

The quantitative CRIP uptake in control animals was obtained from the myocardial specimen corresponding to infarct and remote regions of post-MI animals. 99mTc-CRP = technetium-99m-labeled Cy5.5-RGD imaging peptide; $\mathrm{MI}=$ myocardial infarction; NS = nonsignificant.

$\mathrm{p}<0.05$ to $<0.0001$ compared with control animals.

t $p<0.05$ to $<0.0001$ compared with No Rx-treated animals.

\section{Experimental $\mathrm{Ml}$ in mice}

This experimental protocol was approved by the Institutional Animal Care and Use Committee of the University of California Irvine School of Medicine. In 63 adult Swiss Webster male mice (age, 3 months; body weight, $\approx 50 \mathrm{~g}$ ), the lateral branch of the left coronary artery was ligated under pentobarbital $(75 \mathrm{mg} / \mathrm{kg}$ intraperitoneally) and gas anesthesia (2.0 to $3.0 \%$ isoflurane) to induce $\mathrm{MI}$, as per previous description (21). Successful ligation was verified by visual inspection of myocardial blanching and akinesis or dyskinesis of the apical segment. The chest was closed, and animals were gradually 
weaned from the respirator. Six of the 69 mice were left unmanipulated as disease control mice for comparison with the post-MI mice.

\section{Treatment protocols after $\mathrm{MI}$ in mice}

Captopril (Sigma, St. Louis, Missouri) and losartan (kind gift of Merck, Rahway, New Jersey) were administered in the drinking water, 60 and $20 \mathrm{mg} / \mathrm{kg} /$ day, respectively. Spironolactone (Innovative Research of America, Sarasota, Florida) was administered via a subcutaneous pellet, releasing $1 \mathrm{mg} /$ day, placed under the skin on the back immediately after $\mathrm{MI}$ surgery. The animals received the intervention for 4 weeks beginning immediately after surgery (Table 1 ).

\section{Echocardiography}

Echocardiographic studies were performed in all mice for the evaluation of left ventricular dimensions and function, as reported previously (20). All animals underwent 3 echocardiographic studies; one performed at baseline level, one immediately after $\mathrm{MI}$, and one 4 weeks after MI, just before CRIP imaging. Mice were anesthetized with $2 \%$ isoflurane, and echocardiograms were made (Sequoia, Siemens, Mountain View, California) with a 14-MHz linear probe (15L8, Acuson). An advanced high frame rate imaging technique (Paragon, Siemens) was adopted to increase temporal resolution at a frame rate of $120 \mathrm{fps}$. B-mode images of LV parasternal long-axis, parasternal short-axis, and apical views were digitally acquired at 2 to 3 cardiac cycle lengths. Images of LV short-axis were standardized at 3 levels: base, mid, and apex. M-mode imaging was unified according to the American Society of Echocardiography guidelines for measurements of wall thickness, chamber dimensions, and functional parameters (22). Infarction area and LV area were measured by tracing relative endocardial borders respectively on the long-axis images. Myocardial infarction percentage was calculated as: infarction area/LV area $\times 100$. Left ventricular cavity dimensions, ejection fraction, strain, and rotation were calculated (23).

\section{Radionuclide imaging with ${ }^{99 m} T c-C R I P$}

The technetium-99m-labeled Cy5.5-RGD imaging peptide ( $\mathrm{AH} 110863$, kind gift of GE Healthcare, Oslo, Norway) is a $2.5-\mathrm{kDa}$ peptide conjugated to both a fluorescent cyanine dye Cy5.5 and the chelating agent cPN216, which is linked for radiolabeling of the peptide with $99 \mathrm{mTc}$. The peptide comprises 10 amino acids, contains an RGD motif, and has a bicyclic structure formed by a disulfide and a thioether bridge. The dye moiety and the chelating agent are conjugated to the peptide by the side-chains of lysine residues at the $\mathrm{N}$ - and C-terminal ends, respectively. The technetium-99m-labeled Cy5.5-RGD imaging peptide, by RGD-based targeting, binds to myofibroblasts (20) and is labeled with 99mTc for radionuclide imaging. For radiolabeling, $50 \mu \mathrm{g}$ of $99 \mathrm{mTc}-\mathrm{CRIP}$ was dissolved in $50 \mu \mathrm{l}$ 
of methanol and then added to the freeze-dried kit. A total of $1.0 \mathrm{ml}$ of $99 \mathrm{mTcO} 4-$ was added to the compound and left at room temperature for 20 to $30 \mathrm{~min}$. Instant thinlayer chromatography confirmed radiopurity of more than $90 \%$. Then, $3.7 \pm 0.2 \mathrm{mCi}$ (137 $\pm 7.4 \mathrm{MBq}$ ) of $99 \mathrm{mTc}-\mathrm{CRIP}$ was injected intravenously through the tail vein. At $3.5 \mathrm{~h}$ after 99mTc-CRIP administration, mice were sacrificed with an overdose of pentobarbital (150 $\mathrm{mg} / \mathrm{kg}$ intraperitoneally). Hearts were carefully dissected and planar images of ex vivo hearts were acquired for 15 min in $128 \times 128$ matrix with the use of a low-energy, highresolution pinhole collimator, mounted in a dual-head micro single-photon emission computed tomography gamma camera (X-SPECT, Gamma Medica, Inc., Northridge, California). Thereafter, hearts were cut into apical, middle, and basal slices. The quantitative CRIP uptake was determined with a gamma scintillation counter (1480 Wizard 3", Wallace \& Co., Monrovia, California). We also determined CRIP biodistribution and radiation burden to lung, liver, spleen, and kidney.

\section{Histological evaluation of myocardial specimens}

After completion of the nuclear studies, the 3 bread-loaf slices were washed in phosphate-buffered saline, fixed in $4 \%$ paraformaldehyde in phosphate-buffered saline for 24 $h$, processed, and embedded in paraffin; then, 4- $\mu \mathrm{m}$ sections were prepared. After deparaffinization and rehydration, sections were stained with hematoxylin \& eosin, Masson's trichrome, and picro-Sirius red, as reported previously (20). In picro-Sirius-stained sections, the relative collagen areas in the infarcted, and remote areas were determined under a microscope $(\times 200)$ coupled to a computerized morphometry system (Quantimet, Leica, Cambridge, United Kingdom). The quality of collagen fibers was investigated with the use of Sirius red polarization microscopy, allowing quantitation of the thick, tightly packed, more mature orange/red fibers and the newly formed, thin, loosely assembled yellow/green fibers (24 and 25). Approximately 4 to 5 fields per section were analyzed in the myocardium. Finally, alpha smooth muscle actin (ASMA) was used to determine myofibroblasts in the infarct area, as reported earlier (20). Alpha smooth muscle actin area was quantified by Quantimet analysis in 4 to 5 fields per section; ASMA uptake was only counted for myofibroblasts, and care was taken not to include ASMA uptake observed in vasculature.

\section{Statistical analysis}

Quantitative radiotracer uptake was calculated as percent total injected dose per gram (\%ID/g) of the tissue. Radiotracer uptake and echocardiographic measurements were expressed as mean \pm standard deviation. In our previous study (20), CRIP uptake was reduced by $>25 \%$ in the captopril group compared with the untreated group and in combination treatment (losartan plus captopril) compared with single therapy (captopril alone) group; as such, we propose that having at least 6 mice per group is sufficient to 
detect, with at least $90 \%$ power and alpha $=0.01$, reductions in the uptake of CRIP of at least $25 \%$ in treated compared with untreated animals, or 2 versus 1 agent groups. This calculation confirms that our current study is well-powered. To determine the statistical significance of differences among treatment groups, we used 1-way analysis of variance followed by post-hoc analysis for multiple comparisons. Because we had a limited number of pre-specified pairwise comparisons, namely comparison of control and untreated animals with uptake among 1, 2, or 3 Rx-treated animals, we used the Bonferroni's method for post-hoc analysis. The correlation between the quantitative tracer uptake and echocardiographic parameters or histological characteristics was evaluated by linear regression analysis. $p$ Values $<0.05$ were considered as statistically significant.

\section{Results}

\section{Uptake of ${ }^{99 m} T c-C R I P$ and pharmacologic intervention}

Maximum CRIP uptake was observed in the infarct zone of the untreated post-MI mice; CRIP uptake was substantially reduced in the treated animals (Table 1, Fig. 1A); no CRIP uptake was observed in the unmanipulated hearts. Scrambled CRIP, which has a deranged $R G D$ sequence, did not show uptake in the infarcted myocardium and demonstrated that CRIP uptake is RGD dependent and specific.

The quantitative CRIP uptake in the myocardial tissue specimens was calculated and represented as percent of injected CRIP dose per gram (\%ID/g) of myocardial tissue. We observed CRIP uptake in the infarcted area to be the greatest in the untreated MI group (No Rx; $2.30 \pm 0.14 \%$ ). There was a significant reduction in CRIP uptake in the infarct area in all treated animals $(1.46 \pm 0.42 \%)$ compared with the untreated animals $(p<0.0001)$. The reduction of uptake was significant even when the animals were treated with a single neurohumoral antagonist (1Rx, $1.71 \pm 0.35 \%, p=0.0002)$ (Fig 1B). Each agent was independently effective; spironolactone $(1.80 \pm 0.44 \% ; p=0.0011)$, captopril (1.72 \pm $0.30 \% ; p=0.0059)$, and losartan (1.58 $\pm 0.28 \% ; p<0.0001$ ) (Table 1$)$.

The use of 2 neurohumoral antagonists $(2 \mathrm{Rx}, 1.31 \pm 0.40 \%)$ influenced the CRIP uptake to a greater extent than a single agent $(1 R x, 1.71 \pm 0.35 \%)$ treatment $(p=0.0005)$ (Fig 1B). Uptake with various combinations was as follows; $\mathrm{CL}(1.37 \pm 0.55 \% ; \mathrm{p}<0.0001)$, SC $(1.28 \pm 0.41 \% ; p<0.0001)$, and SL $(1.29 \pm 0.32 \% ; p<0.0001)$ (Table 1). The combination of all 3 agents further reduced the CRIP uptake (3Rx, $1.16 \pm 0.26 \%)$ in the infarct area (Fig. 1B). The decrease in CRIP uptake in triple-treated animals was significantly lower than 1 -agent treatment $(p=0.0001)$ but was statistically insignificantly reduced compared with 2-agent therapy ( $p=0.33$ ) (Fig. 1).

We found that CRIP uptake in the remote myocardium tended to be increased, although not statistically significant, in the untreated post-MI animals $(0.92 \pm 0.22)$ compared with the unmanipulated control animals with no infarcts $(0.68 \pm 0.16, p=0.077)$ (Fig. 1B). 


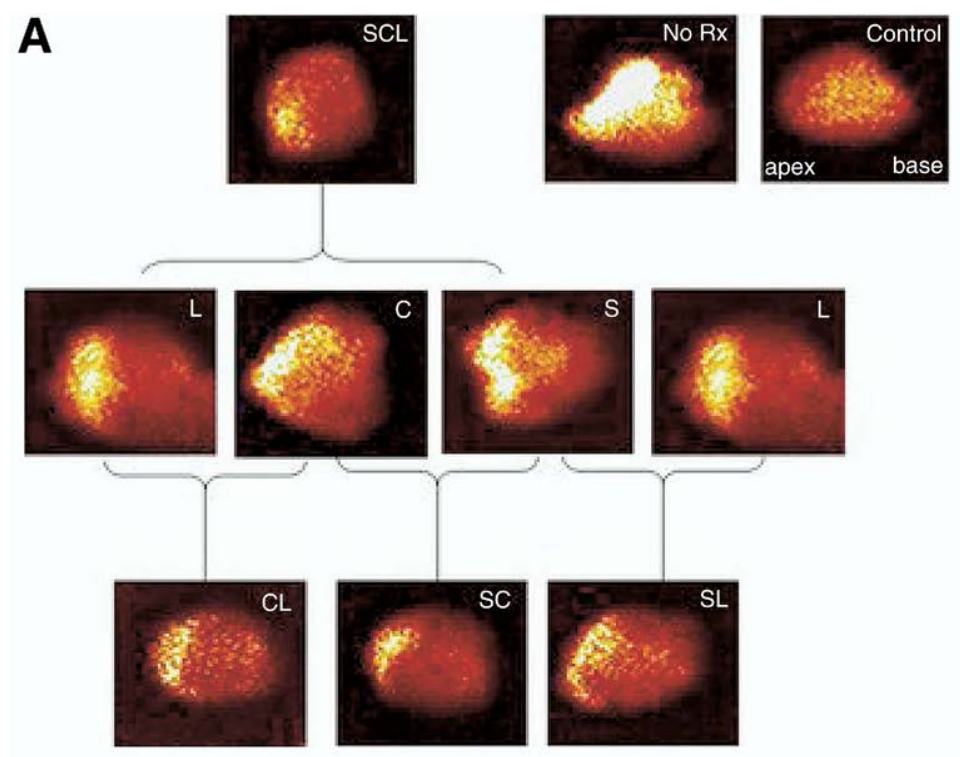

B
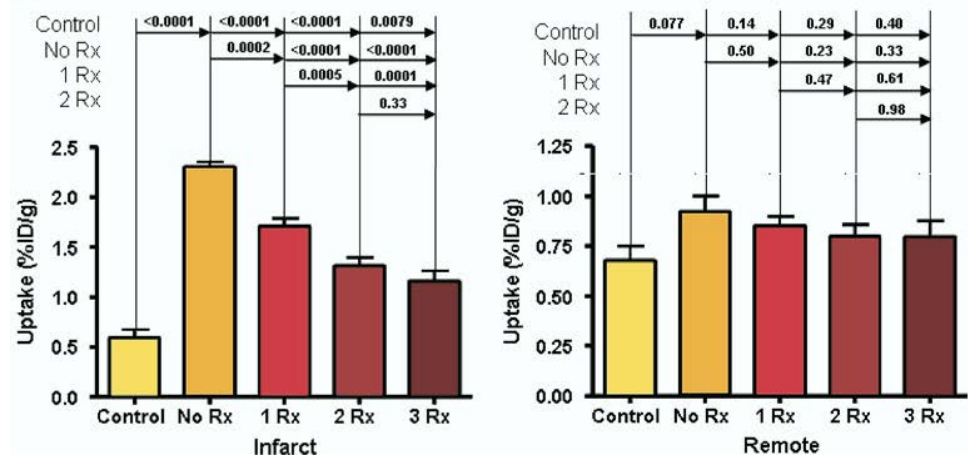

Figure 1. Radiolabeled CRIP Uptake After Neurohumoral Antagonist Treatment in Post-MI Animals

(A) Disease control (unmanipulated) heart with RGD probe shows no radiotracer uptake in ex vivo image of the heart. However, intense ${ }^{99 \mathrm{~m}}$ Tc-CRIP uptake is found in 4-week untreated (no Rx) post-MI animals (upper panel, right). The uptake in the infarcted area is reduced after neurohumoral treatment with solitary agents and combination therapy (middle and lower panels). Losartan ( $L$ ) images are displayed twice (middle panel) for the convenience of comparison. $\mathrm{C}=$ captopril; $\mathrm{S}=$ spironolactone. (B)Quantitative ${ }^{99 \mathrm{~m}} \mathrm{Tc}-\mathrm{CRIP}$ uptake in the infarct (apex, above) and remote (base, below) areas. The\%ID/g uptake in the infarct area is greatest in untreated mice, followed by treatment with $1(1 R x), 2(2 R x)$, and $3(3 R x)$ agents, respectively. On the other hand, the uptake in the remote area shows no significant differences between treated and untreated animals. Quantitative data confirmed the findings of ex vivo images. Data are presented as mean \pm SEM. ${ }^{99 \mathrm{~m}} \mathrm{Tc}$-CRIP $=$ technetium-99m-labeled Cy5.5-RGD imaging peptide; $\mathrm{MI}=$ myocardial infarction;\%ID/g = percent of injected CRIP dose per gram; RGD = Arg-Gly-Asp. 


\section{Echocardiographic examination and remodeling characteristics}

As shown in Table 2, initial MI size was similar in all animal groups. During the 4-week follow-up period, there was a reduction in LV ejection fraction in untreated post-MI (30 $\pm 8 \%$ ) animals compared with unmanipulated disease control animals (64 $\pm 4 \%$, $p<$ 0.0001 ). Neurohumoral antagonists significantly prevented the functional loss (ejection fraction $44 \pm 10 \%$ ), compared with untreated animals (30 $\pm 8 \% ; p=0.0016$ ). Similar to LV ejection fraction, there was a significant decrease in the myocardial strain development in untreated animals (-4.59 $\pm 1.85 \mathrm{U} \%)$ compared with unmanipulated $(-12.00 \pm 2.31$ $\mathrm{U} \%$ ) animals ( $<0.0001)$; loss of myocardial strain was significantly prevented by treatment with neurohumoral agents $(-8.04 \pm 2.4 \mathrm{U} \%, \mathrm{p}=0.0005)$. The strain development in $1 R x(-7.17 \pm 2.28 \cup \%, p=0.0126), 2 R x(-8.28 \pm 2.51 \cup \%, p=0.0011)$, and $3 R x(-9.33 \pm$ $1.7 \cup \%, p<0.0001)$ groups was better preserved compared with the untreated control group. However, the preservation of strain development did not reach statistical significance on comparison of $1 R x$ versus $2 R x(p=0.13)$ or $2 R x$ versus $3 R x(p=0.25)$ agents; $3 R x$ was statistically significantly better than the $1 R x$ intervention $(p=0.0168)$. The results of loss of apical rotation or counterclockwise twist of the LV were similar to the loss of strain development. The rotation in all $\left(0.55 \pm 0.18^{\circ}, \mathrm{p}<0.0001\right), 1 \mathrm{Rx}\left(0.49 \pm 0.17^{\circ}, \mathrm{p}\right.$ $=0.0006), 2 \mathrm{Rx}\left(0.57 \pm 0.19^{\circ}, \mathrm{p}<0.0001\right)$, and $3 \mathrm{Rx}\left(0.63 \pm 0.17^{\circ}, \mathrm{p}<0.0001\right)$ groups was preserved compared with the untreated control group. However, once again, the prevention of rotational loss did not reach statistical significance on comparison of $1 \mathrm{Rx}$ versus $2 R x(p=0.17$ ) or $2 R x$ versus $3 R x(p=0.36$ ) agents; $3 R x$ was statistically significantly better than the $1 R x$ intervention $(p=0.048)$. The LV ejection fraction, strain development, and rotation correlated with\%ID/g CRIP uptake ( $r 2=0.309,=0.397$, and $=0.469$, and $p<0.0001,<0.0001$, and $<0.0001$, respectively) (Fig. 2). 


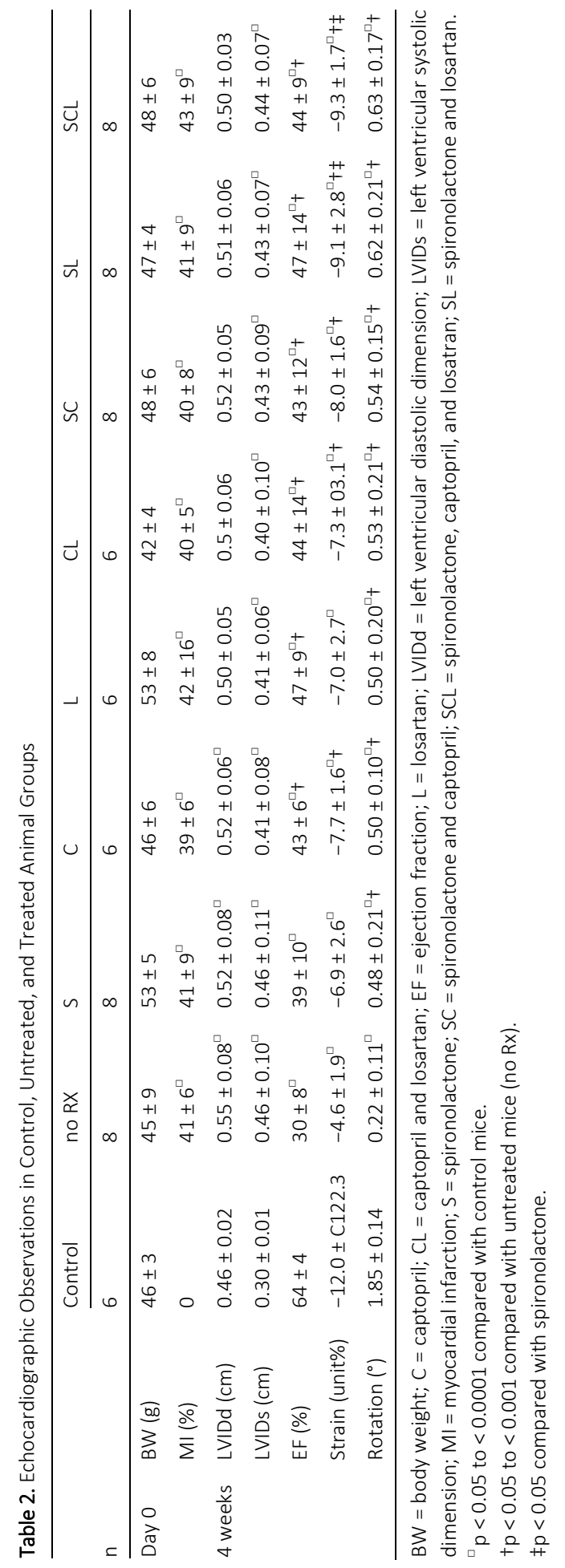


A

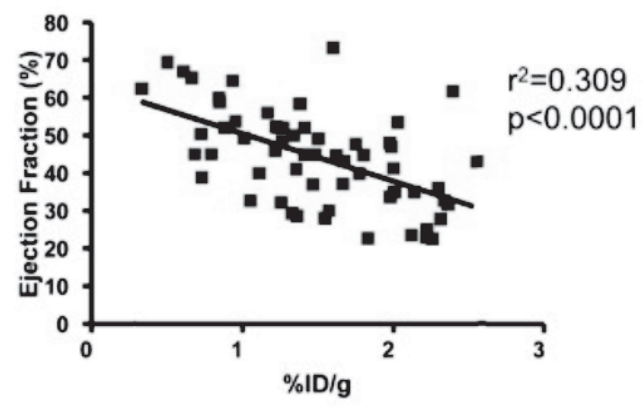

B

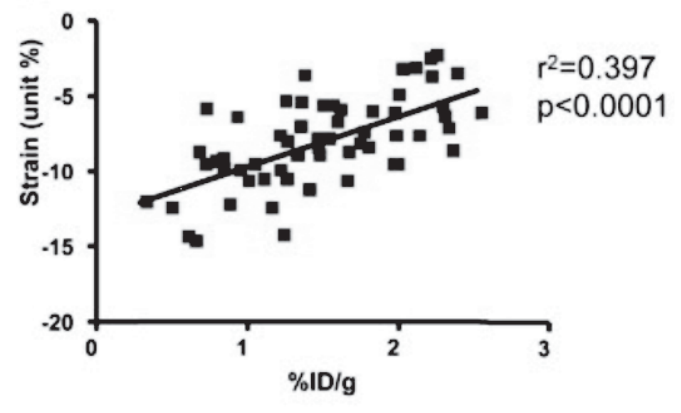

C

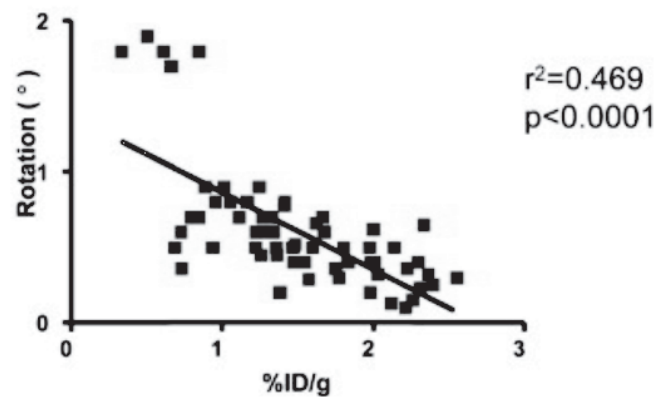

Figure 2. Correlation of radiolabeled ${ }^{99 \mathrm{~m}} \mathrm{TC}$-CRIP Uptake and echocardiographic parameters of remodeling an all animals A significant correlation between CRIP uptake and left ventricular ejection fraction (A), myocardial strain (B), and apical counterclockwise rotation (C) is observed. Abbreviations as in Figure 1. 
Histologic characterization of infarct and remote myocardium and correlation with ${ }^{99 m}$ Tc-CRIP uptake

Masson's trichrome and picro Sirius red staining (Fig. 3) of the myocardial slices allowed the assessment of total collagen content and the composition in thick and thin collagen fibers. The percent collagen content in the infarcted area in untreated animals was $79 \pm$ $4 \%$, which reduced substantially in the treated animals (66 $\pm 8 \%, p<0.0001)$ (Fig. $4 A$,Table 3). The collagen content decreased in animals treated with $1 \mathrm{Rx}(71 \pm 7 \%, \mathrm{p}=$ $0.006), 2 R x(64 \pm 9 \%, p<0.0001)$, or $3 R x(65 \pm 4 \%, p=0.0008)$ agents (Fig 4A). The results were similar for the yellow-green collagen fiber deposition in the infarcted myocardium, suggesting a decrease in new or thin collagen fiber synthesis (Fig. 4C). The new, thin collagen fibers correlated with CRIP uptake in the infarct $(r 2=0.5475, p<0.0001)$ (Fig. 4E). In the remote region, the overall collagen deposition was markedly lower compared with the infarct region. The collagen content of the untreated remote myocardium (No Rx, $2.1 \pm 0.6 \%$ ) was significantly greater than the unmanipulated hearts $(0.73 \pm$ $0.2 \%, p<0.0001$ ) (Fig 4B). A marked decrease in collagen content and yellow-green collagen fiber deposition was observed in the remote region after treatment (Fig. 4D, Table 3); collagen content in treated animals was not different from unmanipulated animals. There was a direct correlation between tracer uptake and thin collagen fiber deposition in the remote region ( $r 2=0.347 ; p<0.0001$ ) (Fig. 4F). 
Control

A
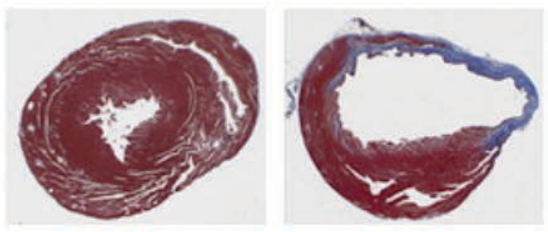

Infarct region

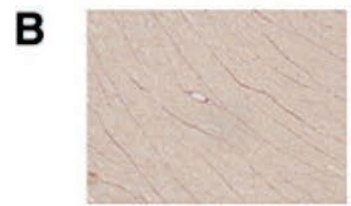

C
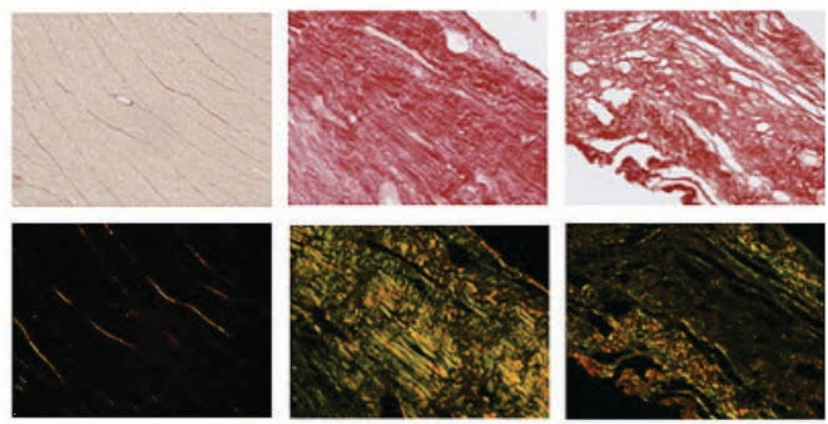

\section{Remote region}
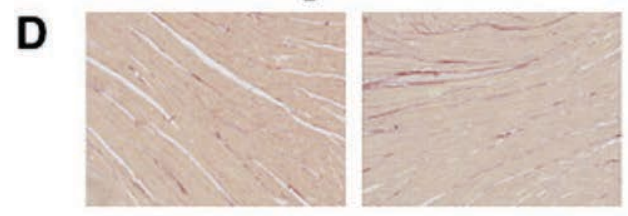

E
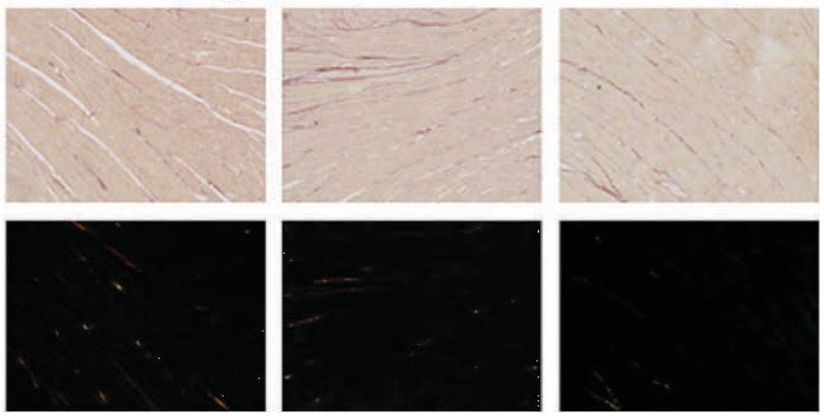

Figure 3. Histopathological Characterization of the Treated and Untreated Animals Masson's trichrome staining was performed for infarct localization in control, untreated, and treated animals (A). In addition, histological staining was performed with picro Sirius red to demonstrate collagen deposition, which on polarized light allowed identification of the quality of collagen, in the infarct ( $B$ and $C$ ) and remote regions ( $D$ and $E$ ). The left column presents control animals, the middle column shows untreated animals (no $\mathrm{Rx}$ ), and the right column shows animals treated with $3 R x$ neurohumoral antagonists (SCL). The remote region shows minimal fibrosis and collagen deposition (D). On the other hand, infarct region shows significant wall thinning $(A)$ and fibrosis with evidence of substantial collagen deposition in untreated animals ( $B$, No Rx). 3Rx-treated animals show reduced fibrosis $(A, S C L)$ and collagen deposition $(B, S C L)$ in the infarcted area. The Sirius staining under polarized light provides distinction of thick red-orange and thin yellow-green collagen fibers in the infarct region (C). SCL = spironolactone, captopril, and losartan. 
A

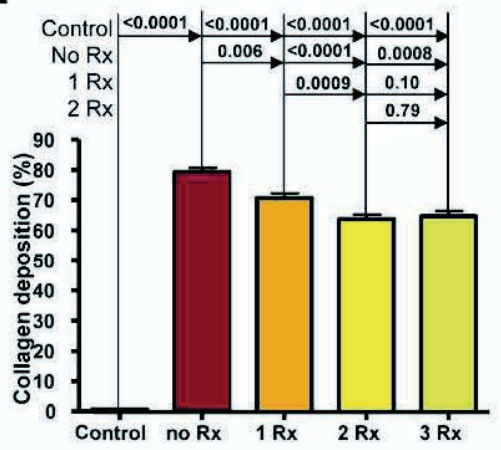

C

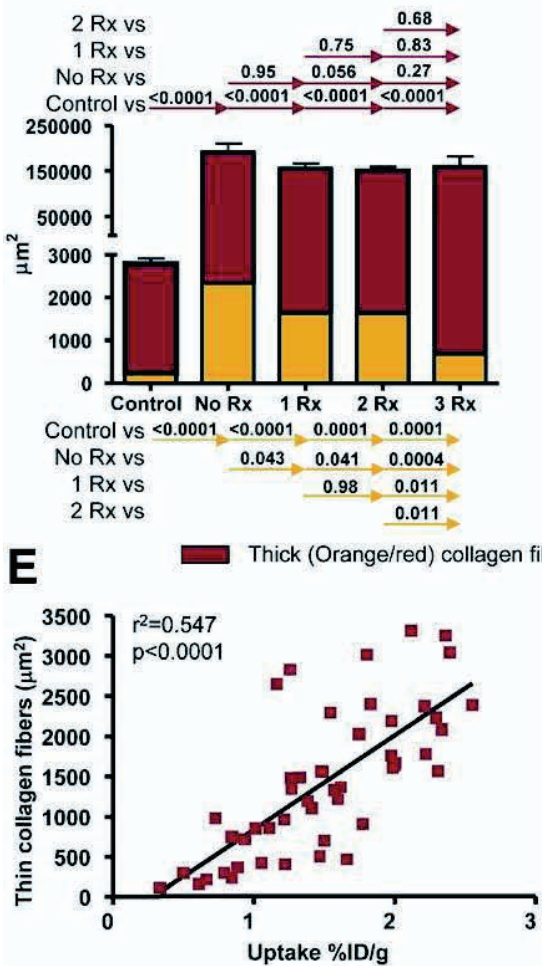

B

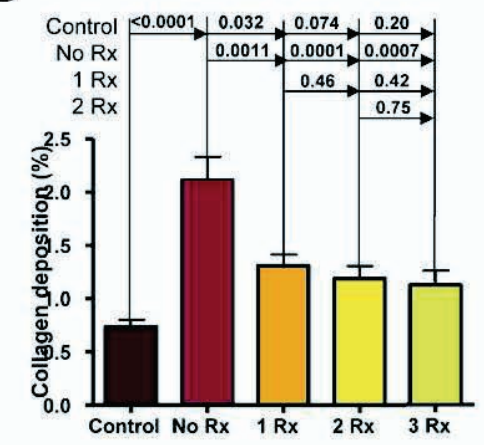

D

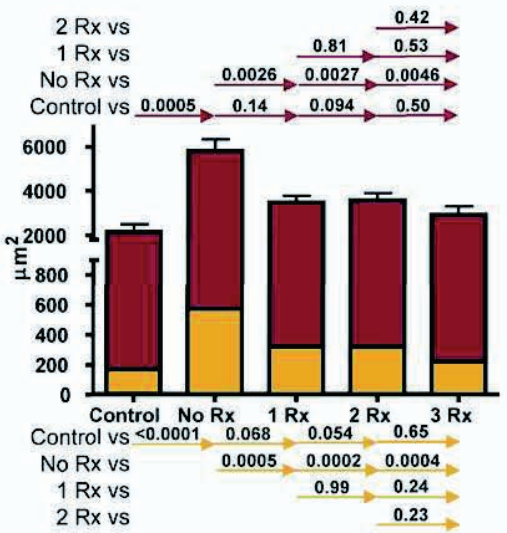

Thin (Yellow/green) collagen fibers

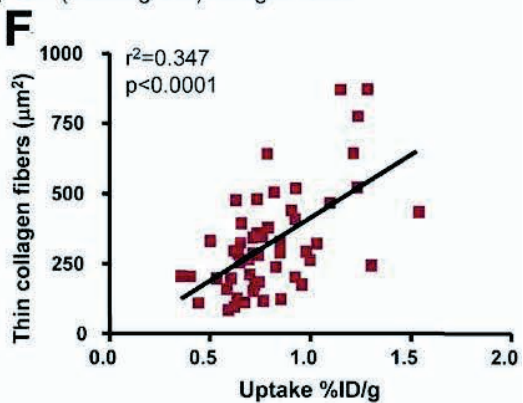

Figure 4. Collagen Fiber Deposition in Infarct and Remote Regions

The total collagen content decreases with treatment in the infarcted (A), as well as the remote (B) areas. Further characterization of the collagen fibers by polarization reveals that the thin or new yellow-green collagen fibers decreased both in the infarct (C) and remote (D) regions after treatment. This indicates cessation of new collagen production and maturation of the collagen fibers after treatment. The prevalence of new collagen fibers paralleled the CRIP uptake and demonstrated a significant direct correlation, both in infarct $(E)$ and remote $(F)$ regions. 
Table 3. The Extent of Collagen Deposition and Myofibroblast Count in Various Animal Groups

\begin{tabular}{|c|c|c|c|c|c|c|c|}
\hline \multirow[t]{2}{*}{ Group } & \multicolumn{4}{|c|}{ Infarct } & \multicolumn{3}{|c|}{ Remote } \\
\hline & Collagen (\%) & $Y G\left(m^{2}\right)$ & $\mathrm{OR}\left(\mathrm{mm}^{2}\right)$ & $\operatorname{ASMA}\left(\mathrm{mm}^{2}\right)$ & Collagen (\%) & $Y G\left(m^{2}\right)$ & $\mathrm{OR}\left(\mathrm{mm}^{2}\right)$ \\
\hline Control & $0.90 \pm 0.14^{\dagger}$ & $221 \pm 79+$ & $2430 \pm 402+$ & $\mathrm{O}^{+}$ & $0.73 \pm 0.18^{\dagger}$ & $170 \pm 66+$ & $2052 \pm 670+$ \\
\hline No $R x$ & $79.3 \pm 4.0^{\square}$ & $2343 \pm 564^{\square}$ & $190026 \pm 47815^{\square}$ & $6.1 \pm 5.2^{\square}$ & $2.11 \pm 0.63^{\square}$ & $571 \pm 235^{\square}$ & $5234 \pm 1491^{\square}$ \\
\hline S & $70.9 \pm 3.8^{\square}+$ & $1452 \pm 505$ & $157337 \pm 24731^{\square}$ & $4.0 \pm 2.8$ & $1.32 \pm 0.78+$ & $323 \pm 268+$ & $3181 \pm 1948+$ \\
\hline C & $71.3 \pm 8.2^{\square}+$ & $1591 \pm 342^{\square}$ & $165676 \pm 31450^{\square}$ & $6.1 \pm 2.0^{\square}$ & $1.29 \pm 0.47 \dagger$ & $328 \pm 111^{\dagger}$ & $3139 \pm 1489+$ \\
\hline $\mathrm{L}$ & $70.1 \pm 8.4^{\square}+$ & $1849 \pm 774^{\square}$ & $145112 \pm 75498^{\square}$ & $4.5 \pm 0.5$ & $1.31 \pm 0.29 \dagger$ & $304 \pm 108^{\dagger}$ & $3155 \pm 889+$ \\
\hline $\mathrm{CL}$ & $68.2 \pm 9.2^{\square}+$ & $2304 \pm 827^{\square}$ & $137198 \pm 46884^{\square}+$ & $4.2 \pm 4.8$ & $1.17 \pm 1.04 \dagger$ & $298 \pm 248+$ & $2950 \pm 2678+$ \\
\hline SC & $61.3 \pm 8.5^{\square}+$ & $1545 \pm 1064^{\square}$ & $154679 \pm 41481^{\square}$ & $1.3 \pm 1.4$ & $1.12 \pm 0.43^{\dagger}$ & $313 \pm 133^{\dagger}$ & $3197 \pm 1575^{\dagger}$ \\
\hline$S L$ & $62.2 \pm 7.3^{\square}+$ & $1248 \pm 937 \dagger$ & $168002 \pm 27358^{\square}$ & $0.7 \pm 0.2+$ & $1.32 \pm 0.33 \dagger$ & $342 \pm 79+$ & $3873 \pm 1150+$ \\
\hline$S C L$ & $64.7 \pm 3.8^{\square}+$ & $689 \pm 249+$ & $159914 \pm 49453^{\square}$ & $1.5 \pm 0.9 \dagger$ & $1.12 \pm 0.41 \dagger$ & $291 \pm 160 \dagger$ & $3321 \pm 1499+$ \\
\hline
\end{tabular}

ASMA = alpha smooth muscle cells; OR = orange-red, thick, crosslinked collagen fibers; YG = yellow-green, thin, new collagen fibers; other abbreviations as in Table 2.

$\square<0.05$ compared with control animals.

$+p<0.05$ compared with No Rx-treated animals.

We performed ASMA staining for quantitation of the myofibroblasts (Fig. 5A). The ASMA-positive area was substantially larger in the untreated animals (No Rx, $6.11 \pm$ $5.17 \mu \mathrm{m} 2)$ compared with the unmanipulated animals $(0.007 \pm 0.01 \mu \mathrm{m} 2, p=0.013)$. Treatment with $1 \mathrm{Rx}(4.91 \pm 2.12 \mu \mathrm{m} 2, \mathrm{p}=0.51), 2 \mathrm{Rx}(2.22 \pm 3.30 \mu \mathrm{m} 2, \mathrm{p}=0.037)$, or $3 \mathrm{Rx}$ $(1.54 \pm 0.92 \mu \mathrm{m} 2, p=0.039)$ interventions substantially restricted the myofibroblast prevalence (Fig. 5B, Table 3). There was a directly proportional relationship between CRIP uptake and ASMA-positive area ( $r 2=0.582, p<0.0001$ ) (Fig. 5C). 
A

Infarct

Remote

B

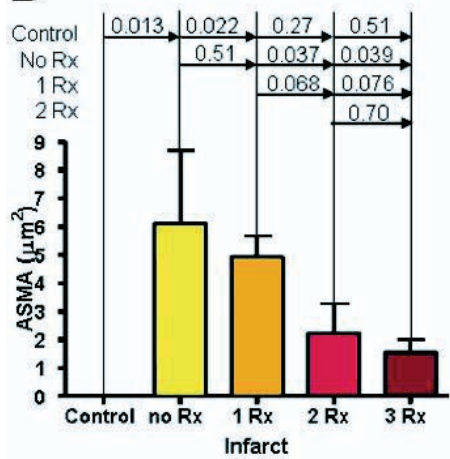

No $\mathrm{Rx}$
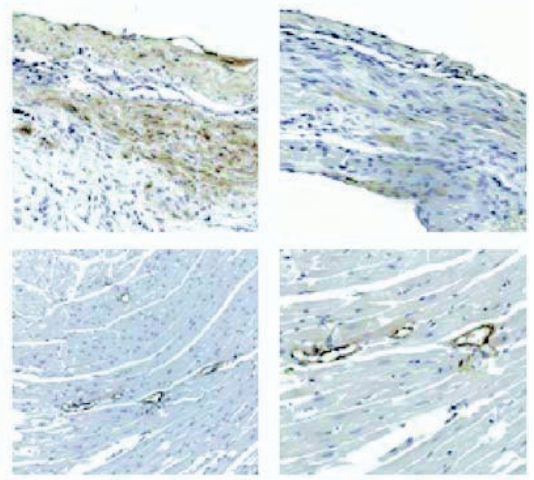

$3 \mathrm{Rx}$

\section{C}

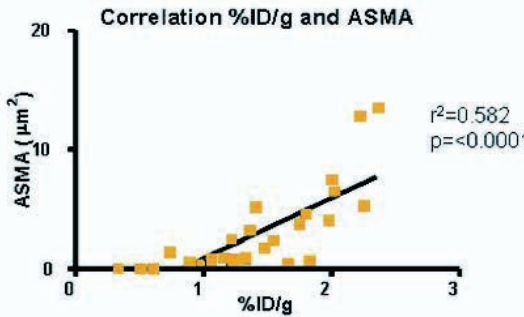

Figure 5. Radiolabeled CRIP Uptake and the Prevalence of ASMA-Verified Myofibroblasts

Immuohistochemical staining with ASMA antibody allowed quantitative assessment of myofibroblasts infiltration in the infarct and remote regions (A). The left column presents control animals, the middle column shows untreated animals (no $\mathrm{Rx}$ ), and the right column shows 3Rx-animals $(\mathrm{SCL})$. There is a marked decrease in number of myofibroblasts in the infarct region in $2 \mathrm{Rx}$ and $3 \mathrm{Rx}$ groups (B). There is a significant direct correlation between the extent of myofibroblasts and the CRIP uptake in infarct region (C). ASMA = alpha smooth muscle actin; other abbreviations as in Figure 1 and Figure 3.

\section{Discussion}

\section{Molecular imaging in myocardial remodeling}

The present study used molecular imaging of myofibroblasts during infarct healing. The radiotracer, CRIP, contains an RGD sequence and binds to myofibroblasts through activated avB3/5 integrins with an affinity of 1 to $3 \mathrm{nmol} / \mathrm{l}$ (20); RGD is also known to commonly target the integrin expression associated with neoangiogenesis early after $\mathrm{MI}$ and 
macrophage influx during infarct healing. We have observed that after 2 weeks of infarct healing, myofibroblasts comprise a predominant target for RGD probes (20). In addition, a specific noncovalent interaction of CRIP also was observed with custom-made DDXsequence contained within pro-collagen I. The previous study using 99mTc-CRIP had demonstrated the feasibility of noninvasively imaging the extent of myofibroblasts prevalence in a post-MI mouse model (20). The CRIP uptake receded over time in the infarct region with substantial resolution over the course of 3 months after the myocardial injury. This previous report also demonstrated that the uptake was significantly reduced at 4 weeks upon treatment with captopril alone or in combination with losartan and proposed that CRIP uptake could monitor the efficacy of neurohumoral antagonists and help identify the optimum level of therapeutic intervention (20). A simultaneously conducted clinical study, using RGD-seeking peptide demonstrated the feasibility of molecular imaging early after $\mathrm{MI}$ (26). The radiotracer uptake after MI predicted the extent of eventual magnetic resonance imaging-verified fibrosis after 1 year. On the basis of these results, the present study investigated the effect of various neurohumoral antagonists, alone or in combination, on the extent of myocardial interstitial alterations in a 4-week post-MI mouse model.

This study confirmed the efficacy of antiangiotensin and antialdosterone intervention on interstitial alterations and demonstrated that combination of 2 neurohumoral antagonists was superior to an individual agent; 3 agents decreased the radiotracer uptake further, albeit not significantly. The radiotracer uptake correlated closely with parameters of depressed myocardial mechanics, the quantitative prevalence of myofibroblasts, and thin fiber collagen deposition, suggesting that treatment with neurohumoral antagonists results in decreased new collagen production. The data obtained in the present study corroborate with information provided by the estimation of collagen synthesis and fragmentation markers in the serum (27- 29). Procollagen type III aminoterminal peptide is decreased in chronic heart failure patients using antiangiotensin and anti-aldosterone treatment $(30,31)$.

\section{What is the optimum therapy?}

The clinical role of ACE-I, ARB, and SARA has been conclusively demonstrated in trials confirming the reduction in morbidity and mortality in patients with overt heart failure (8-11,17,32). These agents also decrease the likelihood of the development of heart failure in patients with asymptomatic decrease in LV ejection fraction $(13,14)$, as well as those predisposed to development of heart failure but normal LV ejection fraction (12, 33). It is also established that antiangiotensin agents in combination are superior to any agent used alone. The combination of ACE-I with ARB in the presence of beta-blockers \pm aldosterone inhibitors reduces the combined end point of mortality (16) and hospitalization for worsening of heart failure (17). The experimental data from the present study confirm the superior effect of combination of 2 agents. However, the addition of the 
third agent did not decrease the uptake significantly. It is encouraging to observe the efficacy of an imaging agent to identify difference in infarct healing, which could be of translational clinical benefit.

\section{Is lack of collagen deposition favorable?}

The present study raises an important question about the relevance of decrease in collagen deposition in the post-infarct patients. Total abolition of collagen deposition, which has been evaluated in clodronate liposome-treated animals (wherein infarct healing becomes defective with minimal collagen deposition and inefficient necrotic myocyte removal) (34), leads to greater tendency for aneurysmal formation and infarct rupture. On the other hand, direct myocardial injection of collagen in experimental infarcts leads to dense collagen deposition and prevents myocardial remodeling (35). Such experiments suggest that the collagen deposition may not necessarily be harmful. However, the use of ACE-I or SARA allows the reduction of collagen deposition as demonstrated by circulating collagen degradation products (30). The present study demonstrated a decrease in collagen deposition with suppression of angiotensin-aldosterone axis. Because neurohumoral antagonists lead to favorable clinical outcomes, conceivably reduced collagen deposition should be of benefit. Although the present study is not capable of explaining these paradoxical observations, it is possible that predominant decrease of collagen content observed in the remote myocardium in the present study, and the collagen fiber maturation (to thick fibers) observed in the infarct region may contribute to a more balanced healing.

\section{Conclusions}

The present study demonstrates that 99mTc-CRIP imaging allows for the evaluation of the efficacy of antiremodeling therapy. It also reconfirms the superiority of combination therapy over a solitary use of neurohumoral antagonists. If proven clinically, molecular imaging of the remodeling processes could facilitate individualizing the treatment for patients susceptible to heart failure.

\section{Acknowledgments}

The imaging agents (99mTc-CRIP and sCRIP) were kindly provided by GE Healthcare, and losartan was a kind gift of Merck \& Co. 


\section{References}

1. K.T. Weber, C.G. Brilla. Pathological hypertrophy and cardiac interstitium: Fibrosis and renin-angiotensinaldosterone system. Circulation, 83 (1991), pp. 1849-1865

2. K.T. Weber, P. Anversa, P.W. Armstrong, et al. Remodeling and reparation of the cardiovascular system. J Am Coll Cardiol, 20 (1992), pp. 3-16

3. B. Pitt. Aldosterone blockade in patients with systolic left ventricular dysfunction. Circulation, 108 (2003), pp. 1790-1794

4. J. Shirani, J. Narula, W.C. Eckelman, N. Narula, V. Dilsizian. Early imaging in heart failure: exploring novel molecular targets. J Nucl Cardiol, 14 (2007), pp. 100-110

5. P.G. Volders, I.E. Willems, J.P. Cleutjens, J.W. Arends, M.G. Havenith, M.J. Daemen. Interstitial collagen is increased in the non-infarcted human myocardium after myocardial infarction. J Mol Cell Cardiol, 25 (1993), pp. 1317-1323

6. J.P. Cleutjens, W.M. Blankesteijn, M.J. Daemen, J.F. Smits. The infarcted myocardium: simply dead tissue, or a lively target for therapeutic interventions. Cardiovasc Res, 44 (1999), pp. 232-241

7. P. Anversa, J.M. Capasso. Cardiac hypertrophy and ventricular remodeling. Lab Invest, 64 (1991), pp. 441-445

8. B. Pitt, P.A. Poole-Wilson, R. Segal, et al. Effect of losartan compared with captopril on mortality in patients with symptomatic heart failure: randomised trial-the Losartan Heart Failure Survival Study ELITE II. Lancet, 355 (2000), pp. 1582-1587

9. The CONSENSUS Trial Study Group. Effects of enalapril on mortality in severe congestive heart failure: Results of the Cooperative North Scandinavian Enalapril Survival Study (CONSENSUS). N Engl J Med, 316 (1987), pp. 1429-1435

10. J.N. Cohn, G. Johnson, S. Ziesche, et al. A comparison of enalapril with hydralazine-isosorbide dinitrate in the treatment of chronic congestive heart failure. N Engl J Med, 325 (1991), pp. 303-310

11. B. Pitt, F. Zannad, W.J. Remme, et al. The effect of spironolactone on morbidity and mortality in patients with severe heart failure: Randomized Aldactone Evaluation Study Investigators. N Engl J Med, 341 (1999), pp. 709-717

12. S. Yusuf, P. Sleight, J. Pogue, J. Bosch, R. Davies, G. Dagenais. Effects of an angiotensin-convertingenzyme inhibitor, ramipril, on cardiovascular events in high-risk patients: The Heart Outcomes Prevention Evaluation Study Investigators. N Engl J Med, 342 (2000), pp. 145-153

13. M.A. Pfeffer, E. Braunwald, L.A. Moye, The SAVE Investigators, et al. Effect of captopril on mortality and morbidity in patients with left ventricular dysfunction after myocardial infarction: Results of the survival and ventricular enlargement trial. N Engl J Med, 327 (1992), pp. 669-677

14. The SOLVD Investigators. Effect of enalapril on survival in patients with reduced left ventricular ejection fractions and congestive heart failure. N Engl J Med, 325 (1991), pp. 293-302

15. B. Pitt, W. Remme, F. Zannad, et al. Eplerenone, a selective aldosterone blocker, in patients with left ventricular dysfunction after myocardial infarction. N Engl J Med, 348 (2003), pp. 1309-1321

16. M.A. Pfeffer, K. Swedberg, C.B. Granger, et al. Effects of candesartan on mortality and morbidity in patients with chronic heart failure: the CHARM-Overall programme. Lancet, 362 (2003), pp. 759-766

17. J.N. Cohn, G. Tognoni. A randomized trial of the angiotensin-receptor blocker valsartan in chronic heart failure. N Engl J Med, 345 (2001), pp. 1667-1675

18. D.M. McNamara, R. Holubkov, K. Janosko, et al. Pharmacogenetic interactions between beta-blocker therapy and the angiotensin-converting enzyme deletion polymorphism in patients with congestive heart failure. Circulation, 103 (2001), pp. 1644-1648

19. K.M. Small, L.E. Wagoner, A.M. Levin, S.L. Kardia, S.B. Liggett. Synergistic polymorphisms of beta1- and alpha2C-adrenergic receptors and the risk of congestive heart failure. N Engl J Med, 347 (2002), pp. 1135-1142

20. S.W.M. van den Borne, S. Isobe, J. Verjans, et al. Molecular imaging of interstitial alterations in remodeling myocardium after myocardial infarction. J Am Coll Cardiol, 52 (2008), pp. 2017-2028 
21. J.W.H. Verjans, D. Lovhaug, N. Narula, et al. Noninvasive imaging of angiotensin receptores after myocardial infarction. J Am Coll Cardiol Cardiovasc Imaging, 1 (2008), pp. 354-362

22. N.B. Schiller, P.M. Shah, M. Crawford, et al. Recommendations for quantitation of the left ventricle by two-dimensional echocardiography: American Society of Echocardiography Committee on Standards, Subcommittee on Quantitation of Two-Dimensional Echocardiograms. J Am Soc Echocardiogr, 2 (1989), pp. 358-367

23. G. Tonti, J. Verjans, G. Pedrizzetti, et al. Measurement of apical torsion in mitochrondrial cardiomyopathy using a novel B-mode, automated tracking algorithm. J Am Coll Cardiol, 45 (2005), p. 305A

24. P. Whittaker, R.A. Kloner, D.R. Boughner, J.G. Pickering. Quantitative assessment of myocardial collagen with picrosirius red staining and circularly polarized light. Basic Res Cardiol, 89 (1994), pp. 397-410

25. D.A. MacKenna, J.H. Omens, A.D. McCulloch, J.W. Covell. Contribution of collagen matrix to passive left ventricular mechanics in isolated rat hearts. Am J Physiol, 266 (1994), pp. H1007-H1018

26. J.W. Verjans, S.L. Wolters, M. Lax, et al. Imaging avb3/b5 Integrin Upregulation in Patients After Myocardial Infarction. Circulation, 116 (2007), p. 113288

27. S.H. Poulsen, N.B. Host, S.E. Jensen, K. Egstrup. Relationship between serum amino-terminal propeptide of type III procollagen and changes of left ventricular function after acute myocardial infarction. Circulation, 101 (2000), pp. 1527-1532

28. M. Cicoira, A. Rossi, S. Bonapace, et al. Independent and additional prognostic value of aminoterminal propeptide of type III procollagen circulating levels in patients with chronic heart failure. J Card Fail, 10 (2004), pp. 403-411

29. J. Radovan, P. Vaclav, W. Petr, et al. Changes of collagen metabolism predict the left ventricular remodeling after myocardial infarction. Mol Cell Biochem, 293 (2006), pp. 71-78

30. F. Zannad, F. Alla, B. Dousset, A. Perez, B. Pitt, Rales Investigators. Limitation of excessive extracellular matrix turnover may contribute to survival benefit of spironolactone therapy in patients with congestive heart failure: insights from the randomized aldactone evaluation study (RALES). Circulation, 102 (2000), pp. 2700-2706

31. F. Zannad, A. Radauceanu. Effect of MR blockade on collagen formation and cardiovascular disease with a specific emphasis on heart failure. Heart Fail Rev, 10 (2005), pp. 71-78

32. J.J. McMurray, J. Ostergren, K. Swedberg, et al. Effects of candesartan in patients with chronic heart failure and reduced left-ventricular systolic function taking angiotensin-converting-enzyme inhibitors: the CHARM-Added trial. Lancet, 362 (2003), pp. 767-771

33. S. Yusuf, K.K. Teo, J. Pogue, et al. Telmisartan, ramipril, or both in patients at high risk for vascular events. N Engl J Med, 358 (2008), pp. 1547-1559

34. M.J. van Amerongen, M.C. Harmsen, N. van Rooijen, A.H. Petersen, M.J. van Luyn. Macrophage depletion impairs wound healing and increases left ventricular remodeling after myocardial injury in mice. Am J Pathol, 170 (2007), pp. 818-829

35. W. Dai, L.E. Wold, J.S. Dow, R.A. Kloner. Thickening of the infarcted wall by collagen injection improves left ventricular function in rats: a novel approach to preserve cardiac function after myocardial infarction. J Am Coll Cardiol, 46 (2005), pp. 714-719 


\section{CHAPTER 8}

\section{Noninvasive molecular imaging of cell death in myocardial infarction using}

\section{${ }^{111}$ in-GSAO}

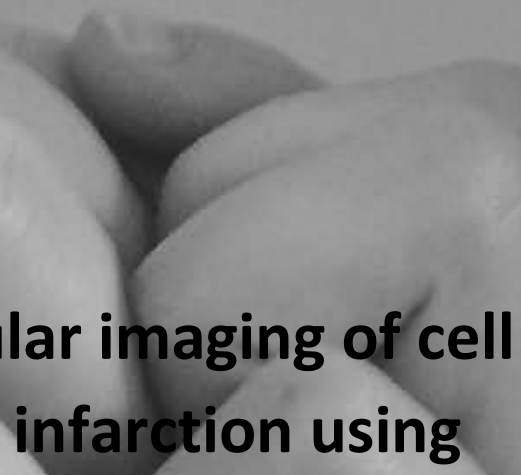

N. Tahara*, H.R. Zandbergen*, H.J. de Haas*, A.D. Petrov, R. Pandurangi, T. Yamaki,

J. Zhou, T. Iamizumi, R.H.J.A. Slart, M. Dyszlewski, T. Scarabelli, A. Kini,

C. Reutelingsperger, N. Narula, V. Fuster and J. Narula

*contributed equally

Nature - Scientific Reports, October 2014 

Acute insult to the myocardium is associated with substantial loss of cardiomyocytes during the process of myocardial infarction. In this setting, apoptosis (programmed cell death) and necrosis may operate on a continuum. Because the latter is characterized by the loss of sarcolemmal integrity, we propose that an appropriately labeled tracer directed at a ubiquitously present intracellular moiety would allow non-invasive definition of cardiomyocyte necrosis. A trivalent arsenic peptide, GSAO (4-(N-(S-glutathionylacetyl)amino)phenylarsonous acid), is capable of binding to intracellular dithiol molecules such as HSP9O and filamin-A. Since GSAO is membrane impermeable and dithiol molecules abundantly present intracellularly, we propose that myocardial localization would represent sarcolemmal disruption or necrotic cell death. In rabbit and mouse models of myocardial infarction and post-infarct heart failure, we employed In-111labelled GSAO for noninvasive radionuclide molecular imaging. ${ }^{111}$ In-GSAO uptake was observed within the regions of apoptosis seeking agent- ${ }^{99 \mathrm{~m}} \mathrm{Tc}$-Annexin A5 uptake, suggesting the colocalization of apoptotic and necrotic cell death processes.

Cell death plays a central role in various cardiovascular diseases. Two morphologically distinct modes of cell death - apoptosis (a programmed process characterized by enzymatic degradation and clean removal of the cell) and necrosis (an uncontrolled process characterized by cell swelling, membrane rupture and spill of its contents) have been reported to contribute to the myocardial tissue loss. It is being increasingly realized that apoptosis and necrosis, rather than being entirely independent, may operate on a continuum, at least in response to noxious stimuli. ${ }^{1-3}$

Numerous strategies have been proposed for the detection of cell death early after onset of ischemia within the time window amenable to intervention. Most experience for the recognition of apoptosis has been obtained with single photon emission computed tomography (SPECT) imaging using ${ }^{99 \mathrm{~m}} \mathrm{Tc}$-Annexin A5 (AA5). ${ }^{99 \mathrm{~m}} \mathrm{Tc}-\mathrm{AA} 5$ targets externalized phosphatidylserine (PS) on membranes of cells with active apoptotic signaling ${ }^{4}$ and the clinical feasibility of ${ }^{99 \mathrm{~m}} \mathrm{Tc}-\mathrm{AA} 5$ imaging has been demonstrated in the setting of myocardial infarction ${ }^{5,6}$, transplant rejection ${ }^{7}$ and heart failure. ${ }^{8}$ On the other hand, several radiotracers targeting necrotic cells through membrane disruption have been developed. ${ }^{9-11}$ Notably, antimyosin antibody imaging has been successfully employed for the detection of myocardial necrosis associated with myocardial infarction ${ }^{9}$, myocarditis $^{9}$, heart failure ${ }^{12}$ and cardiac allograft rejection. ${ }^{12}$ However due to technical disadvantage of prolonged wait time for imaging, the necrosis-avid radiotracers have not become popular.

4-(N-(S-glutathionylacetyl)amino)phenylarsonous acid (GSAO) GSAO labeled with fluorophores and radionuclides has been used for targeting of cell death in culture, in tumor-bearing mice, and in mice with experimental brain trauma. ${ }^{13-15}$ The principle behind GSAO cell death targeting is displayed in figure 1. GSAO is a tripeptide with a trivalent arsenic moiety. The arsenic group binds to dithiols, which are abundantly present in intracellular milieu and virtually absent from the extracellular space. ${ }^{16}$ GSAO cannot reach its intracellular target molecules in intact cells, because it is not able to negotiate 
across the cell membrane. ${ }^{17}$ Cell membrane $y$-glutamyl transferase (GGT) is upregulated during ischemic stress and other situations, splice off GSAO's glutamyl residue and allow cellular entrance of GSAO's metabolite GCAO (4-(N-(S-cysteinylglycylacetyl)amino) phenylarsonous acid). ${ }^{17}$ However, when radioactive or fluorescent reporter molecules are attached to glutamyl residue of GSAO it is not able to bind to GGT, rendering the molecule membrane impermeable. ${ }^{13}$ After the necrotic process sets in and the sarcolemmal integrity is lost; and GSAO gains free entry to the intracellular microenvironment. Of numerous dithiol-bearing intracellular targets including filamin A, eukaryotic translation elongation factor 2, and protein disulfide isomerase (PDI), HSP90 is most widely present. ${ }^{13}$ HSP9O comprises approximately $2 \%$ of the intracellular protein content and increases by two-threefold in response to acute stress including ischemia. ${ }^{18}$

a

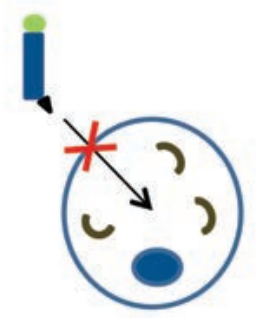

Intact GSAO is membrane impermeable

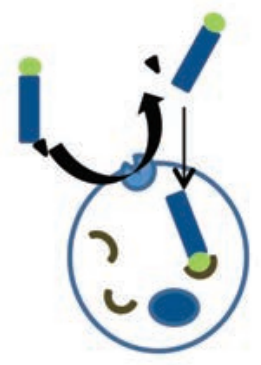

Membrane bound GGT (on proliferating and stressed cells) splices off glutamyl residue and allows cell entrance of metabolite GCAO
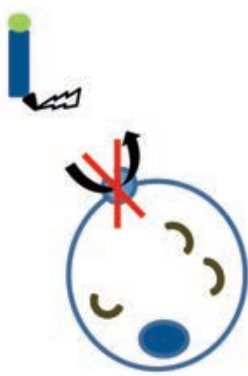

Binding of fluorescent or radioactive reporter molecules to GSAO prevents binding to GGT and thereby prevents all access over intact membrane b

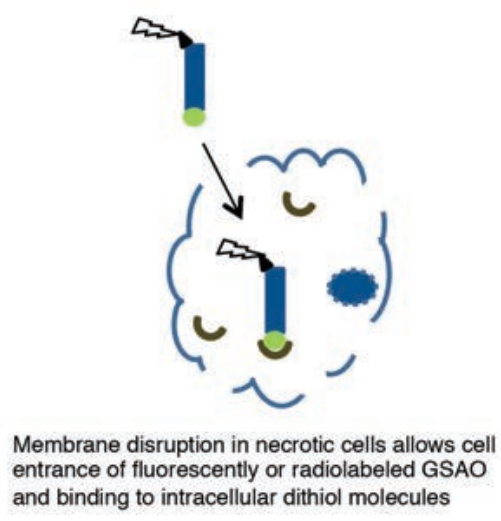

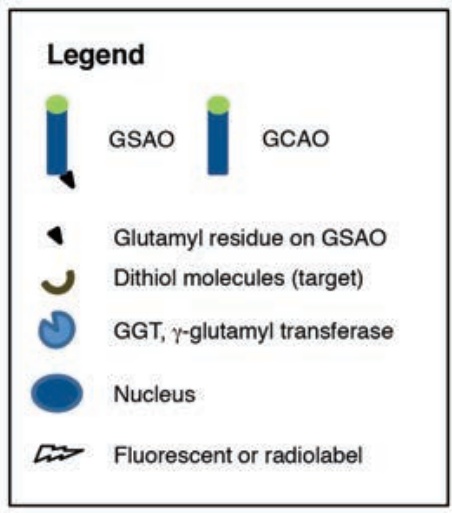

Figure 1. Targeting mechanism of GSAO.

GSAO binds to dithiol molecules, which are abundant in the intracellular space and virtually absent in the extracellular space. When labelled with radioactive or fluorescent reporter molecules, GSAO cannot negotiate across the cell membrane of healthy cells (a) but gains free access through disrupted membranes of dying cells (b). Thus, GSAO accumulates in dying cells. 
In this study we evaluated feasibility of cell death imaging using ${ }^{111}$ In-GSAO in mouse and rabbit models of acute $\mathrm{MI}$ and a mouse model of chronic MI. In addition, a subgroup of animals, serial SPECT/CT imaging using ${ }^{99 \mathrm{~m}}$ Tc-AA5 and ${ }^{111} \mathrm{In}-\mathrm{GSAO}$ was performed to determine the relationship between these two modes of cell death in the setting of myocardial ischemia and reperfusion. Fluorescent GSAO and AA5 were employed in another subgroup of animals for pathological characterization of the mode of cell death. In addition, ${ }^{111}$ In-GSCA (4-(N-(S-glutathionylacetyl)amino)benzoic acid), which is identical to ${ }^{111}$ In-GSAO except for replacement of the arsenic group with a carboxylic acid group, was used as a negative control compound in radionuclide experiments.

\section{Results}

\section{Fluorescence microscopic characterization of GSAO uptake}

Uptake characteristics of GSAO were evaluated and compared with AA5 in mice with acute $\mathrm{MI}$ and sham-operated mice. Experimental acute $\mathrm{MI}$ was induced by 30-minute coronary ligation followed by 30 minutes of reperfusion. Sham procedure was identical, except that coronary ligation was not performed. Mice with acute $\mathrm{MI}(\mathrm{n}=3)$ and shamoperated mice ( $n=3$ ) were injected Cy5.5-labelled GSAO and Oregon Green-labeled AA5. Qualitative fluorescent microscopic analysis of heart sections showed that cardiomyocytes of sham-operated mice were positive neither for GSAO nor for AA5. In contrast, in ischemia/reperfusion-injured hearts, cardiomyocytes positive for GSAO and AA5 were observed (Fig. 2a-c). Interestingly, all GSAO-positive cardiomyocytes bound AA5 (Fig. 2ac), suggesting that necrosis in the reperfused myocardium coexists with apoptotic signaling. Not all AA5 positive cardiomyocytes had taken up GSAO (Fig. 2c). 

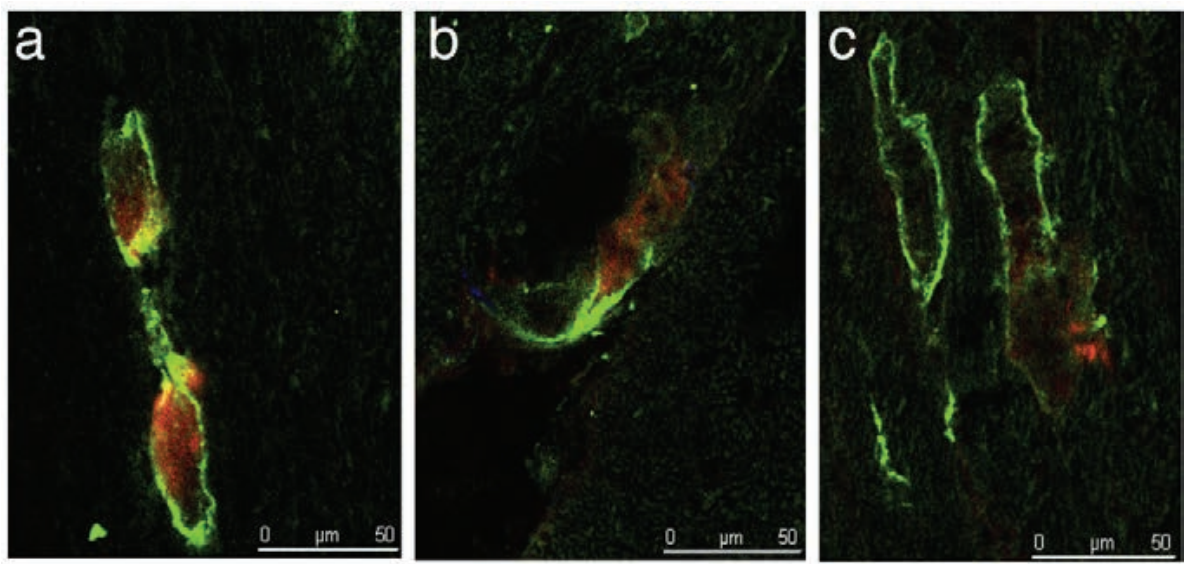

Figure 2. Characterization of target binding of GSAO.

Tissue sections of acute MI mice, injected fluorescently labeled GSAO and AA5. (a,b) GSAO accumulation (red) was only observed in cells with AA5-positive cell membranes (green). (c) All cells AA5-positive cells were not GSAO positive.

\section{Radiolabelled GSAO imaging in acute myocardial infarction}

Acute $\mathrm{MI}$ was induced in rabbits and mice by coronary ligation for 40 and 30 minutes, respectively. Reperfusion was achieved by removal of the suture and ${ }^{111}$ In-GSAO was administered thirty minutes later. Three hours thereafter, in vivo micro-SPECT/micro-CT imaging was performed, animals were sacrificed and hearts were explanted. Next, ex vivo SPECT/CT and planar cardiac imaging were performed. Then, rabbit hearts were cut into 32 small pieces and mice hearts were sectioned in three short axis slices: basal (remote area), middle (border zone) and apical (infarct). Radiotracer uptake was quantified by $\mathrm{p}$-counting, and myocardial pieces were histopathologically characterized.

In vivo SPECT/CT imaging in MI rabbits $(n=10)$ revealed intense uptake of ${ }^{111}$ In-GSAO in the apical area (Fig. 3a). Ex vivo SPECT/CT and planar imaging (Fig 3b) of explanted hearts confirmed intense apical radiotracer uptake. $\gamma$-counting confirmed that the ${ }^{111} \mathrm{In}$ GSAO uptake in the myocardial infarct was markedly higher than the ${ }^{111}$ In-GSAO uptake in the remote region $(1.10 \pm 0.45 \% \mathrm{ID} / \mathrm{g}$ vs. $0.03 \pm 0.01 \% \mathrm{ID} / \mathrm{g}, \mathrm{P}=0.005, \mathrm{Fig}$. 3c). 
a
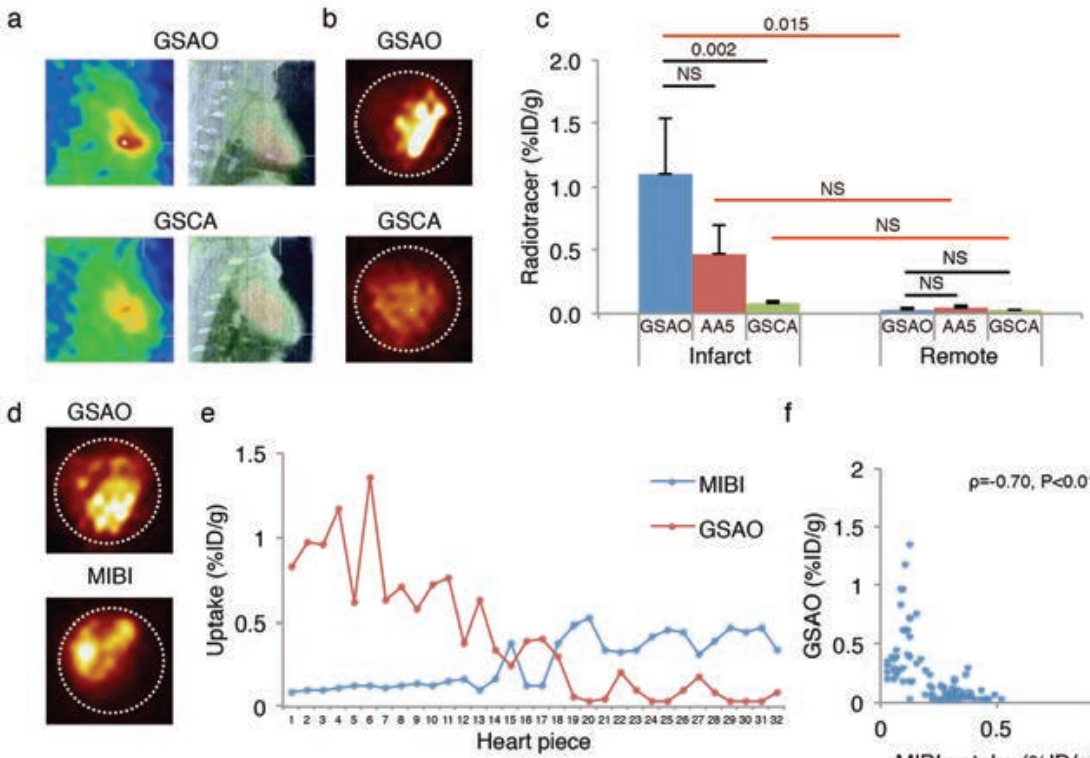

f
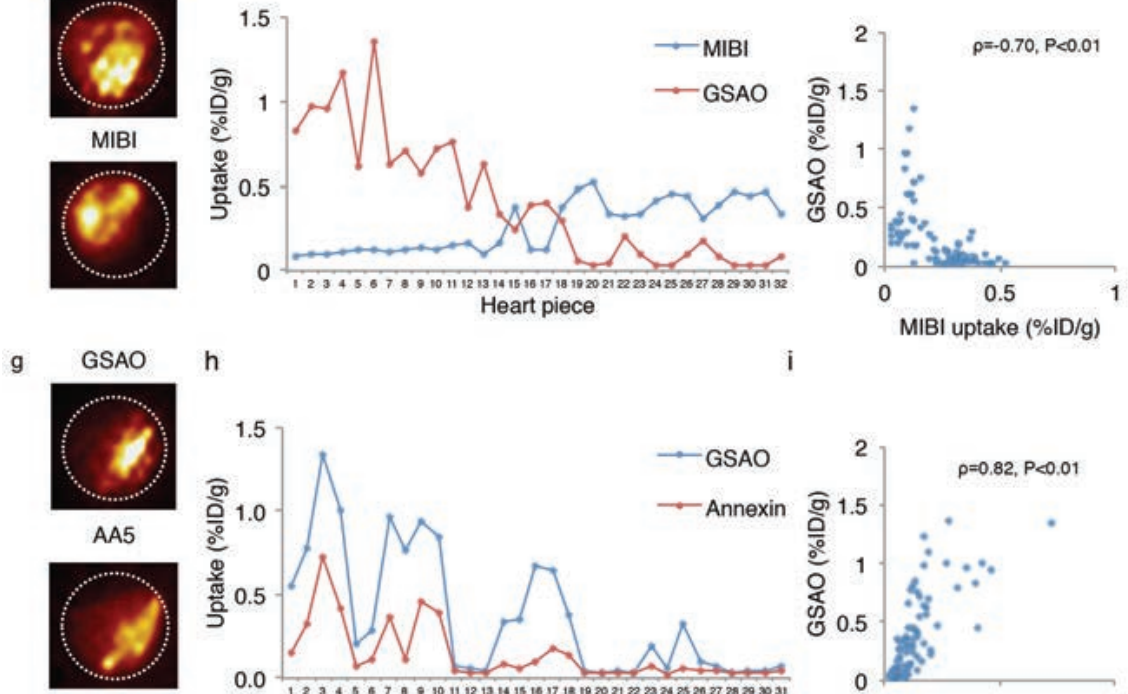

h

\section{i}
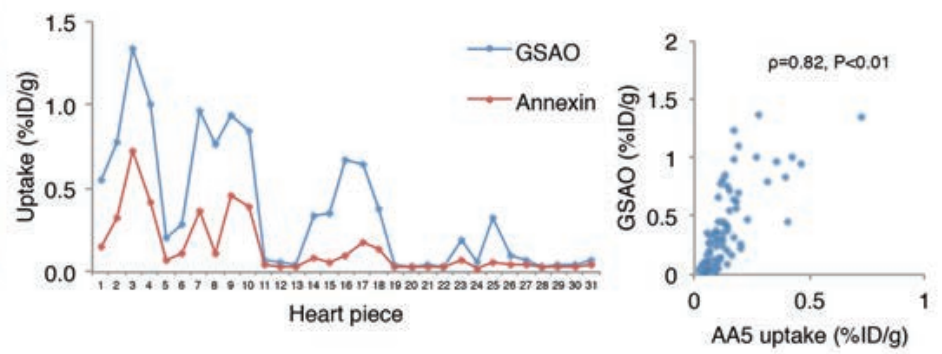

Figure 3. ${ }^{111}$ In-GSAO imaging, quantification and comparison with ${ }^{99 \mathrm{~m}} \mathrm{Tc}-$ Annexin A5 and ${ }^{99 \mathrm{~m}} \mathrm{Tc}-\mathrm{MIBI}$ in rabbits with acute myocardial infarction.

(a) In vivo SPECT (left panels), and fused SPECT/CT images (right panels) in acute MI rabbits revealed intense ${ }^{111}$ In-GSAO uptake (top) and only modest uptake of radiotracer control compound ${ }^{111}$ In-GSCA (bottom). (b) Ex vivo planar images confirmed intense ${ }^{111}$ In-GSAO uptake (top) and modest ${ }^{111}$ In-GSCA (bottom) uptake. (c) $\gamma$ counting of myocardial sections confirmed ${ }^{111}$ In-GSAO uptake in infarct was higher than in remote area and higher than ${ }^{111}$ In-GSCA uptake in infarct. Although low, ${ }^{111}$ In-GSCA uptake in infarct was higher than remote. Difference Between ${ }^{111}$ In-GSAO and ${ }^{99 m}$ Tc-AA5 uptake did not reach significant uptake. Black horizontal lines denote Kruskal-Wallis ANOVAs + Bonferroni correction, and red lines denote Wilcoxon signed rank tests + Bonferroni correction. (d) Serial imaging revealed that ${ }^{111}$ In-GSAO uptake (top) was predominantly localized in the region of the ${ }^{99 \mathrm{~m}} \mathrm{Tc}-\mathrm{MIBI}$ perfusion defect (bottom). (e) $\mathrm{\gamma}$-counting of myocardial sections showed high ${ }^{111}$ In-GSAO uptake in sections with low MIBI uptake and vice versa. (f) A significant inverse Spearman's correlation between uptake of ${ }^{111} \mathrm{In}-\mathrm{GSAO}$ and ${ }^{99 \mathrm{~m}} \mathrm{Tc}-\mathrm{MIBI}$ was observed. (g) Serial planar imaging in acute MI rabbit demonstrates similar uptake region and higher uptake of ${ }^{111} \mathrm{In}$-GSAO (top) when compared with ${ }^{99 \mathrm{~m}} \mathrm{Tc}$-AA5 (bottom). (h) $\mathrm{y}$-counting of myocardial sections showed high ${ }^{111}$ In-GSAO uptake in sections with high ${ }^{99 \mathrm{~m}} \mathrm{TC}-\mathrm{AA} 5$ uptake and vice versa (i). A significant Spearman's correlation between uptake of ${ }^{111} \mathrm{In}-\mathrm{GSAO}$ and ${ }^{99 \mathrm{~m}} \mathrm{Tc}-\mathrm{AA5}$ was observed. 
To study the specificity of ${ }^{111}$ In-GSAO, the myocardial uptake of negative control compound GSCA was evaluated in acute MI rabbits $(n=5)$. In vivo (Fig. 3a) and ex vivo SPECT/CT imaging and planar imaging (Fig. $3 \mathrm{~b}$ ) revealed very low cardiac uptake of GSCA; only slightly increased infarct uptake was observed. This was confirmed by $\gamma$ counting; when compared with ${ }^{111}$ In-GSAO, ${ }^{111}$ In-GSCA uptake in the infarct area was significantly lower $(0.07 \pm 0.03 \% \mathrm{ID} / \mathrm{g}, \mathrm{P}=0.002$, Fig $3 \mathrm{c})$. Although low, GSCA uptake in the infarct area was higher than in the remote area $(0.03 \pm 0.01 \% \mathrm{ID} / \mathrm{g}, \mathrm{P}=0.043)$. This demonstrated that the trivalent arsenical group was necessary for targeting of dying cells. Moreover, myocardial ${ }^{111}$ In-GSAO uptake in an unmanipulated control animal was similar to ${ }^{111}$ In-GSAO uptake in the spared myocardium of the MI rabbits $(0.01 \%$ ID/g vs. $0.03 \pm$ $0.01 \% \mathrm{ID} / \mathrm{g})$, further supporting specificity of GSAO.

To evaluate localization of cardiac ${ }^{111}$ In-GSAO uptake, three of ten animals receiving ${ }^{111}$ In-GSAO also received the myocardial perfusion tracer ${ }^{99 \mathrm{~m}}$ Tc-sestamibi (MIBI), immediately after coronary ligation. Serial in vivo SPECT/CT imaging confirmed that ${ }^{111}$ In-GSAO uptake was localized in the infarct zone as identified by ${ }^{99 \mathrm{~m}} \mathrm{Tc}-\mathrm{MIBI}$ perfusion defect. Similarly, ex vivo serial SPECT/CT imaging and serial planar imaging (Fig. 3d) revealed uptake of ${ }^{111}$ In-GSAO in the infarct zone as shown by ${ }^{99 m}$ Tc-MIBI perfusion defect. In each animal, $y$-Counting of ${ }^{99 m} \mathrm{Tc}-\mathrm{MIBI}$ and ${ }^{111}$ In-GSAO in myocardial sections confirmed increased uptake of ${ }^{111}$ In-GSAO in the perfusion defects as sections with low MIBI uptake

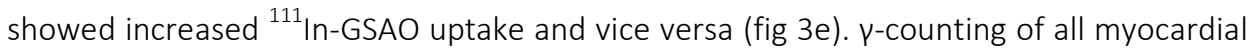
sections from the animals receiving both ${ }^{111} \mathrm{In}-\mathrm{GSAO}$ and ${ }^{99 \mathrm{~m}} \mathrm{Tc}-\mathrm{MIBI}$ revealed an inverse correlation between ${ }^{111}$ In-GSAO uptake and ${ }^{99 \mathrm{~m}} \mathrm{Tc}-\mathrm{MIBI}$ uptake ( $\rho=-0.70, p<0.01$, Fig 3f).

Of ten animals receiving ${ }^{111} \mathrm{In}-\mathrm{GSAO}$, three also received ${ }^{99 \mathrm{~m}}$ Tc-AA5. Serial in vivo SPECT/CT imaging with ${ }^{111} \mathrm{In}-\mathrm{GSAO}$ and ${ }^{99 \mathrm{~m}}$ Tc-AA5 revealed similar area of increased uptake; ${ }^{111}$ In-GSAO uptake was more intense than ${ }^{99 \mathrm{~m}} \mathrm{Tc}-\mathrm{AA} 5$ uptake This was confirmed by ex vivo serial SPECT/CT imaging and ex vivo serial planar imaging (Fig. 3g). Quantitative tracer uptake paralleled the imaging data. In each animal, in sections with increased 99mTc-AA5 uptake, ${ }^{111}$ In-GSAO uptake was also increased (fig. 3h). Using all myocardial sections in all animals receiving ${ }^{111} \mathrm{In}-\mathrm{GSAO}$ and ${ }^{99 \mathrm{~m}} \mathrm{Tc}-\mathrm{AA} 5$, a strong correlation between uptake of the radiotracers was observed $(\rho=0.82, p<0.01$, Fig 3i). Like the fluorescence experiments, this supports that secondary necrosis after apoptotic signaling may be the dominant mode of cell death in myocardial infarction. The ${ }^{111}$ In-GSAO uptake in the infarct zone was higher than ${ }^{99 \mathrm{~m}} \mathrm{Tc}$-AA5 uptake, although the results did not reach statistical significance, due to the small sample size $(1.10 \pm 0.45 \%$ ID/g vs. $0.47 \pm 0.23 \%, P=0.47)$. Uptake in the remote area was low for both tracers $(0.03 \pm 0.01 \%$ ID/g vs. $0.04 \pm 0.02 \%$ $\mathrm{ID} / \mathrm{g}, \mathrm{P}=\mathrm{NS}$ ).

After $y$-counting myocardial pieces from infarct, border and remote zones were histologically characterized. Representative examples of the stainings are given in figure 4a. H\&E staining revealed hypereosinophilic change and contraction band necrosis in the myocardial sections from the infarct area; the morphological changes were less frequent in the border zone. The remote area was morphologically normal. The rate of apoptosis 
as shown by terminal deoxyribonucleotide transferase TdT-mediated nick-end labeling (TUNEL) staining (\%area positive, \%AP) was higher in the infarct $(2.83 \pm 1.42 \% \mathrm{AP})$ and border zone $(2.55 \pm 1.84 \% \mathrm{AP})$ than in the remote zone $(0.02 \pm 0.0 \% \mathrm{AP}, \mathrm{P}<0.001$ for both). The \%caspase positive area was higher in the infarct $(1.04 \pm 0.48 \% \mathrm{AP})$ than in the remote zone $(0.46 \pm 0.48 \% A P, P=0.054) .{ }^{111}$ In-GSAO uptake in the myocardial sections showed a direct correlation with TUNEL ( $\rho: 0.743, P<0.001$, Fig. $4 b)$ and Caspase-3 $(\rho=0.533$, $P<0.001$, Fig. 4c) stains, further supporting that necrosis as shown by GSAO is secondary after apoptotic signaling.
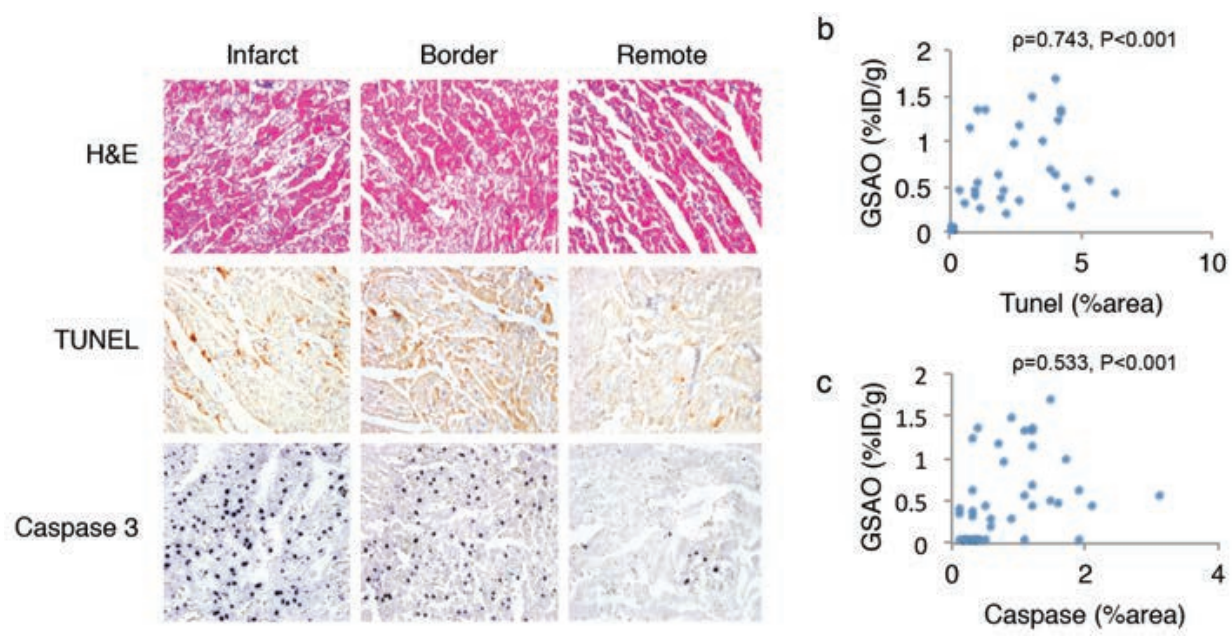

Figure 4. Histopathological characterization of ${ }^{111}$ In-GSAO uptake in rabbit heart after experimental acute myocardial infarction.

H\&E staining demonstrates clear signs of tissue damage in infarct and border zone, whereas the remote zone is morphologically normal. TUNEL and Caspase-3 stains demonstrate substantial apoptosis in the infarct zone, whereas positive cells are rarely seen in the remote area. Significant Spearman's Rank correlations between ${ }^{111}$ In-GSAO uptake and apoptotic signaling as shown by TUNEL (b) and Caspase-3 (c) stains were observed.

The non-target organ distribution of ${ }^{111}$ In-GSAO and ${ }^{111}$ In-GSCA demonstrated kidney to be the major organ of radiation burden and urine major route of excretion; all other organs revealed minimum burden for both radiotracers (Supplementary Fig.1a). Serial blood samples from six animals revealed bi-exponential blood clearance with an initial fast component $T_{1 / 2} \alpha$ of $5.3 \mathrm{~min}$ followed by a slower component $T_{1 / 2} \beta$ of $9.7 \mathrm{~h}$; the plateau phase was approached at 15 min (Supplementary Fig. 1b).

In vivo SPECT/CT imaging in mice after acute $\mathrm{MI}(\mathrm{n}=6)$ demonstrated high ${ }^{111}$ In-GSAO uptake; the use of $\mathrm{CT}$ allowed precise localization of the radioactivity in the infarcted region of the heart (Fig. 5a,b). Specific apical uptake was confirmed by ex vivo SPECT/CT and planar imaging (Fig. 5c). Imaging experiments revealed absence of specific ${ }^{111} \mathrm{In}$ GSAO uptake in control animals. ${ }^{111}$ In-GSAO uptake in the infarct area $(2.56 \pm 2.75 \% \mathrm{ID} / \mathrm{g})$ was markedly higher than in remote myocardium $(0.48 \pm 0.22 \% \mathrm{ID} / \mathrm{g}, \mathrm{P}=0.028)$ and 20 -fold 
higher than apical uptake in unmanipulated control animals $(n=5,0.13 \pm 0.02 \% \mid \mathrm{D} / \mathrm{g}$; $p=0.004$, Fig. $5 d)$.

a

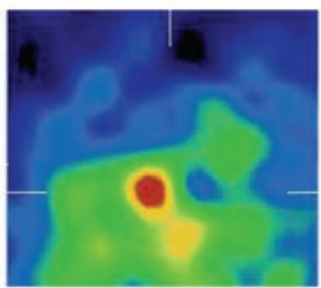

b

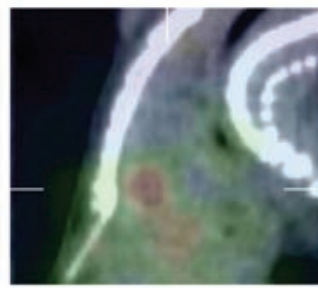

0.028
C

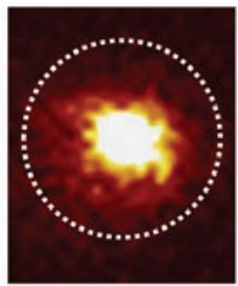

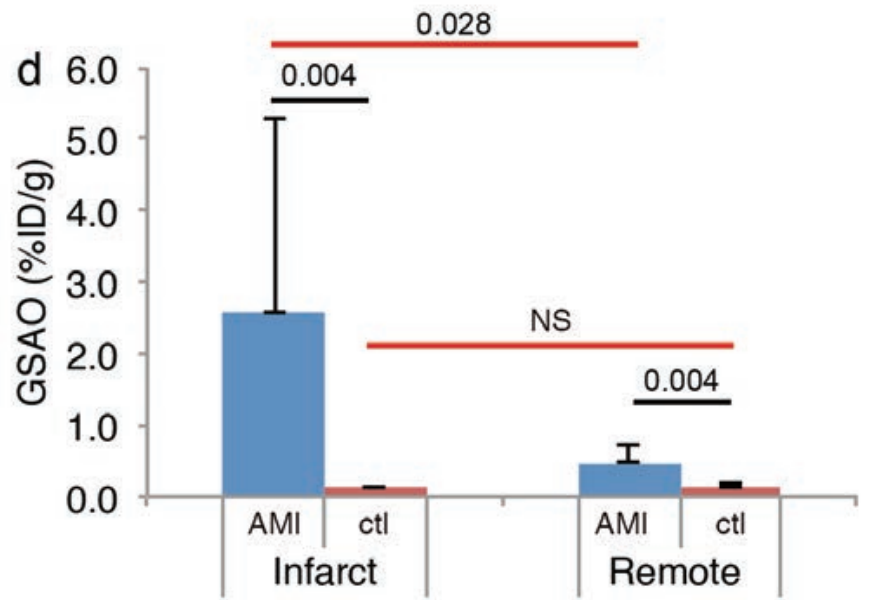

Figure 5. In vivo and ex vivo ${ }^{111}$ In-GSAO imaging and quantification in mice with acute myocardial infarction.

In vivo SPECT (a), and fused SPECT/CT images (b) in acute $\mathrm{MI}$ rabbits revealed intense cardiac ${ }^{111}$ In-GSAO uptake. (c) Ex vivo planar images confirmed intense ${ }^{111}$ In-GSAO uptake. (d) $p$-counting of myocardial sections confirmed ${ }^{111}$ In-GSAO uptake in infarct (apex) was higher than manyfold higher than in remote area and higher than apical ${ }^{111}$ In-GSAO uptake in unmanipulated control animals. Black horizontal lines denote Mann-Whitney $U$ tests and red lines denote Wilcoxon signed rank tests.

\section{Radionuclide imaging in a post-MI HF model}

${ }^{111}$ In-GSAO uptake was also evaluated in post-MI HF mouse model, in which the LCA territory was not reperfused. Subgroups underwent imaging experiments at different time points. Also, a disease control group consisting of animals that did not undergo infarction surgery before ${ }^{111}$ In-GSAO imaging, and a radiotracer control group consisting of animals receiving negative control compound ${ }^{111}$ In-GSCA imaging at 2 weeks post-MI were used. 
${ }^{111}$ In-GSAO uptake in the HF mice was markedly lower than in AMI mice, and could not be detected by in vivo SPECT/CT imaging. Quantification of radionuclide uptake in short axis slices confirmed that infarct uptake of ${ }^{111}$ In-GSAO in mice at 2 weeks post-MI $(n=6)$ was higher than in the five disease control animals that did not undergo infarction surgery $(0.42 \pm 0.17 \% \mathrm{ID} / \mathrm{g}$ vs. $0.13 \pm 0.02 \% \mathrm{ID} / \mathrm{g}, \mathrm{P}=0.025$, Fig 6$)$; remote uptake was not statistically different $\left(0.25 \pm 0.12 \% \mathrm{ID} / \mathrm{g}\right.$ vs. $0.14 \pm 0.04 \% \mathrm{ID} / \mathrm{g}, \mathrm{P}=1.0$, Fig 6). Moreover, ${ }^{111} \mathrm{In}$ GSAO uptake at $2 \mathrm{w}$ post-MI was significantly higher than uptake of the negative control compound ${ }^{111} \mathrm{In}-\mathrm{GSCA}$ in the infarct and remote area $(0.04 \pm 0.02 \% \mathrm{ID} / \mathrm{g}, \mathrm{P}<0.001$ and $0.04 \pm 0.03 \% \mathrm{ID} / \mathrm{g}, \mathrm{P}=0.004$, respectively)

Moreover, when compared with uptake at 2 weeks post-MI, ${ }^{111}$ In-GSAO uptake showed a trend of decline at 4 weeks $(n=6$, infarct $0.28 \pm 0.09 \% \mathrm{ID} / \mathrm{g}, P=1.0$; remote $0.22 \pm 0.04 \% \mathrm{ID} / \mathrm{g}, \mathrm{P}=1.0$, Fig 6 ) and 12 weeks ( $=8$, infarct: $0.19 \pm 0.07 \% \mathrm{ID} / \mathrm{g}, \mathrm{P}=0.332$; remote: $0.15 \% \pm 0.1 \% \mathrm{ID} / \mathrm{g}, \mathrm{P}=1.0$, Fig 6 ) although differences were not statistically significant.

Infarct

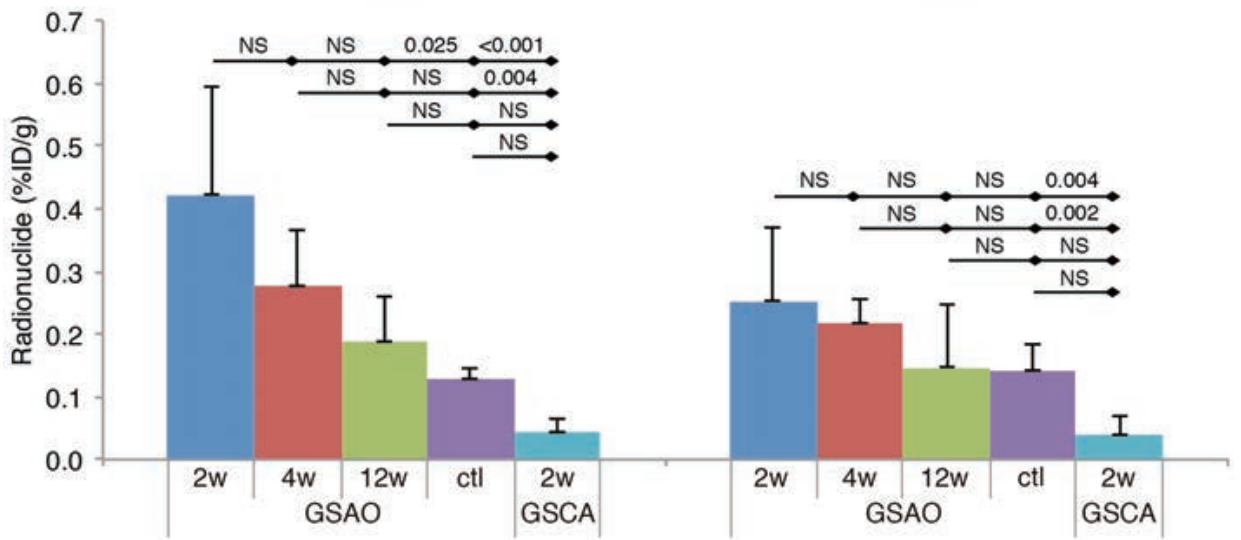

Figure 6. Quantification of ${ }^{111}$ In-GSAO uptake in mice with chronic myocardial infarction.

$\Gamma$-counting revealed ${ }^{111}$ In-GSAO uptake in the infarct area at 2 weeks; uptake was significantly higher than ${ }^{111}$ InGSAO uptake in unmanipulated control animals and ${ }^{111}$ In-GSCA uptake at $2 \mathrm{w}$ post-MI in controls. A trend of declining ${ }^{111}$ In-GSAO uptake over time was observed, but did not reach statistical significance. ${ }^{111}$ In-GSAO uptake in remote area was higher than ${ }^{111}$ In-GSCA uptake at $2 \mathrm{w}$ post-MI. Results of Kruskal-Wallis ANOVAs + Bonferroni correction are shown.

\section{Discussion}

Here we demonstrate cell death imaging in acute myocardial infarction using ${ }^{111}$ In-GSAO. The specificity of the radiotracer was confirmed by the localization of ${ }^{111}$ In-GSAO in the infarct zone as shown by ${ }^{99 \mathrm{~m}} \mathrm{Tc}-\mathrm{MIBI}$. This was further supported by the lack of uptake of fluorescently labeled GSAO in sham-operated animals outside of the regions directly 
damaged by the suture. The lack of GSCA uptake in our radionuclide studies confirmed that the trivalent arsenic group on GSAO is responsible for its targeting characteristics. ${ }^{15,29,30}$ The radiotracer showed favorable pharmacokinetic profile with rapid blood clearance and low background uptake in most organs.

In the 1990's evidence of apoptotic signaling in myocardial infarction accumulated ${ }^{19-}$ ${ }^{22}$, sparking the discussion over the relative importance of the apoptotic and necrotic forms of cell death. In 2000, ${ }^{99 m}$ Tc-AA5 SPECT in patients with acute myocardial infarction revealed intense uptake in the entire region of the perfusion defect ${ }^{5}$. This observation was provocative as ${ }^{99 m}$ Tc-AA5 was believed to identify apoptotic cell death but the infarct area was traditionally expected to be necrotic. It was subsequently proposed that the ischemic insult may be initiated as apoptosis but conclude with secondary necrosis. Ischemic loss of ATP production during myocardial infarction would preclude completion of the energy-dependent apoptosis program. ${ }^{23}$ Restoration of blood flow by reperfusion may either interrupt the apoptotic process to allow cell salvage ${ }^{6,24}$ or resume the process of apoptosis in critically damaged cells. ${ }^{20,22}$ The latter may even be augmented further by production of radical oxygen species ${ }^{25}$, or intracellular calcium overload. ${ }^{26}$ These changes may also contribute to secondary necrosis by opening of the mitochondrial permeability transition pore. ${ }^{27-30}$ It is conceivable that the apoptotic process initiated by noxious stimuli may not follow classical picture of physiologic apoptosis observed during normal turnover of skin or mucosal cells.

The feasibility of cell death imaging using fluorescently labeled and radiolabelled GSAO has recently been demonstrated in tumor-bearing mice and mice with experimental brain trauma. ${ }^{13-15}$ GSAO accumulation was characterized by fluorescent microscopy in various cell models of apoptotic and in explanted brains and tumors after in vivo GSAO administration. GSAO was observed intracellularly and colocalized with propidium iodide and Sytox blue, standard markers of membrane disruption, which supported GSAO uptake in secondarily necrotic cells. Similarly, our fluorescent experiments showed that GSAO accumulated intracellularly and only occurred in AA5+ cells, thereby supported the notion that necrosis followed apoptotic signaling in the setting of acute myocardial infarction.

In vivo and ex vivo ${ }^{111}$ In-GSAO imaging in rabbits and mice with acute myocardial infarction showed intense dual serial imaging with ${ }^{99 m}$ Tc-AA5 and ${ }^{111}$ In-GSAO in rabbits after acute myocardial ischemia and reperfusion revealed the same area of uptake. At a segment level, ${ }^{111}$ In-GSAO uptake showed the same pattern as ${ }^{99 m}$ Tc-AA5 and a strong correlation between uptakes of the two tracers was shown. In addition, ${ }^{111}$ In-GSAO correlated with presence of Caspase-3 and TUNEL staining, classic markers of apoptosis. This shows that not only does secondary necrosis after apoptotic signaling occur in myocardial ischemia and reperfusion, it may play a dominant role. As in rabbits, high ${ }^{111} \mathrm{In}$ GSAO uptake was seen in mice with acute myocardial infarction. However, ${ }^{111}$ In-GSAO uptake in post-myocardial infarction HF model in mice was too low to be detected by micro-SPECT/CT imaging. The most important explanation for this is the lower rate of 
necrotic cell death in heart failure. ${ }^{31-33}$ It is tempting to speculate that the more benign circumstances such as lesser energy depletion and ROS cause lower transition from apoptosis to necrosis in this setting. In fact, the apoptotic process may remain suspended in chronic heart failure. ${ }^{34}$

A number of imaging tracers for myocardial necrosis have been clinically evaluated. Like ${ }^{111}$ In-GSAO, most tracers exploited membrane disruption, the hallmark of necrosis as a target. Tc-99 labelled antimyosin antibody was the most widely studied necrosis tracer. Antimyosin antibody imaging has been successfully employed for the detection of myocardial necrosis associated with myocardial infarction ${ }^{9}$, myocarditis ${ }^{9}$, heart failure ${ }^{12}$ and cardiac allograft rejection. ${ }^{12}$ However, because of long circulation time of the radiolabelled antibody, imaging was not feasible for up to 6-12 hours after administration of the agent. ${ }^{99 \mathrm{~m}}$ Tc-pyrophosphate on the other hand, showed maximal myocardial uptake at 24-72 hours after injection, although necrosis imaging at 3 hours post-administration was feasible, maximum myocardial uptake was at 24-72h. Also, this tracer required residual blood flow, which precluded uptake in the infarct center. ${ }^{35} 99 \mathrm{~m}$ Tc-glucarate can be used to necrotic cells in myocardial infarction ${ }^{11,36}$, and has good imaging characteristics but in the setting of myocardial infarction, use is limited to a clinical window of $>9$ hours after onset as its target, histone bodies, quickly wash out of the tissue. ${ }^{11}$ Late gadolinium-enhanced MRI has also been used to assess cardiac cell death; the contrast medium accumulates in necrotic cells through the disrupted membranes. However, its specificity is limited, as in all instances of increased extracellular space such as cardiac edema, fibrosis, sarcoidosis and amyloidosis. ${ }^{37-39}{ }^{111}$ In-GSAO appears to have advantages over the previously evaluated cell death imaging techniques. First, ${ }^{111}$ In-GSAO requires membrane disruption, the hallmark of necrotic or late-apoptotic cell death, for reaching its intracellular targets. Therefore it does not suffer from the lack of specificity of lategadolinium enhanced MRI. Moreover, its rapid blood clearance results in feasibility of early imaging of cell death using ${ }^{111}$ In-GSAO. This gives ${ }^{111}$ In-GSAO an advantage over ${ }^{99 \mathrm{~m}}$ Tc-antimyosin and ${ }^{99 \mathrm{~m}}$ Tc-pyrophosphate. HSP90, the main target of GSAO, functions in complexes. This may allow for targeting over a longer time period, giving ${ }^{111}$ In-GSAO an edge over ${ }^{99 \mathrm{~m}} \mathrm{Tc}$-glucarate. However, this has to be evaluated in follow-up studies.

Micro-SPECT/CT imaging using ${ }^{111}$ In-GSAO can be used to visualize necrotic cell death in acute myocardial infarction. The uptake in the HF model was too low to allow imaging. Because the GSAO uptake reflects membrane permeabilization, and occurs predominantly in AA5 positive cells, we propose that secondary necrosis is a dominant mode of cell death in the setting of myocardial ischemia and reperfusion. 


\section{Methods}

\section{Materials}

Cy5.5 GSAO and DTPA-GSAO were prescribed as described previously ${ }^{13}$ and were a kind gift of Covidien Imaging Solutions (Hazelwood, Missouri, United States). DTPA-GSAO and DTPA-GSCA were labeled with In-111 as described previously. ${ }^{13}$ The other used materials were of standard analytical grade.

\section{Ethical statement}

The experimental protocols followed the Guidelines for the Care and Use of Laboratory Animals established by the National Institutes of Health (NIH Publication No. 85-23, revised 1996) and was approved by the Institutional Laboratory Animal Care and Use Committees at the University of California, Irvine and University of Maastricht, Maastricht, The Netherlands.

\section{Experimental myocardial infarction in mice}

For fluorescence experiments, C57BI6/j (Age: 3 months, weight: 50g, Jackson Laboratories, Sacramento, CA) and for radionuclide studies Swiss-Webster mice (Age: 3 months, weight $\sim 50 \mathrm{~g}$, Charles River, Wilmington, MA) were used. MI was induced under isoflurane anesthesia (2-3\%) using a stereomicroscope (Leica MZ FL III, Leica, Switzerland) as described previously. ${ }^{40}$ Animals were placed on a heating pad in the supine position, intubated under direct laryngoscopy, and mechanically ventilated using a small animal respirator (tidal volume, $1.0 \mathrm{ml}$; rate, 120 breaths/min, Harvard Apparatus, Holliston, MA). After a minimum thoracotomy, the anterior descending branch of the left coronary artery was ligated with a 6.0-silk suture 3 to $4 \mathrm{~mm}$ below the tip of the left atrium. Successful ligation was verified by visual inspection of the LV apex for myocardial blanching, indicating interruption in coronary flow. For induction of acute $\mathrm{Ml}$ experiments, the suture was removed after 30 minutes to induce reperfusion. The sham operation was identical, but the coronary artery was not ligated. Acute $\mathrm{MI}$ and sham animals remained under anesthesia for ensuing radionuclide imaging or fluorescence experiments. For induction of chronic MI, the ligation was not released. In these animals, the chest cavity was closed in layers with 6.0-silk and the skin was closed with 4.0-silk sutures. Thereafter, animals were gradually weaned from the respirator and put back into their cages until imaging procedure. 


\section{Experimental acute myocardial infarction in rabbits}

Acute experimental MI was induced by the occlusion of left anterior descending coronary artery (LAD) in New Zealand White male rabbits (weight, 3.0-3.5kg) as described previously. ${ }^{24,41}$ All rabbits were anesthetized with a mixture of ketamine and xylazine (100 mg/ml, 10:1 vol/vol; 2.0 to $3.0 \mathrm{ml}$ subcutaneously). Surgical tracheostomy was performed, and ventilation was maintained with a volume-cycled rodent respirator (Harvard Apparatus, Holliston, MA) provided positive pressure ventilation at $50 \mathrm{~mL} / \mathrm{cycle}$ and a respiratory rate of $50 \mathrm{cycles} / \mathrm{min}$. After surgical procedure, anesthesia was maintained on $3-4 \%$ isoflurane Briefly, the heart was exposed through parasternal thoracotomy, and the pericardium was fenestrated. The LAD was identified and a monofilament suture was placed at the site. The LAD was occluded by tightening the snare created by passing suture through a polyethylene tube. The snare was removed after 40 minutes of occlusion to induce reperfusion. Lead II or III of the electrocardiogram was continuously monitored during the experiments to confirm myocardial ischemia. Animals remained anesthetized for ensuing radionuclide imaging experiments.

\section{Fluorescence experiments in mice}

Acute MI mice and sham-operated mice were injected with Cy5.5-labelled GSAO at 5 minutes before ischemia and 1 hour before sacrifice, respectively. All mice received Oregon Green-labeled AA5 10 minutes before sacrifice. Of each fluorescent probe a dosage of $2.5 \mathrm{mg} / \mathrm{kg}$ was used. Acute $\mathrm{Ml}$ animals were sacrificed after $30 \mathrm{~m}$ reperfusion, sham mice at 1 hour after sacrifice hearts were snap-frozen in liquid nitrogen, $7 \mu \mathrm{m}$ thick frozen sections were obtained, dried and mounted in 4,6-diamino-2-phenylindole (DAPI) containing medium and examined with a confocal scanning laser microscope (Bio-Rad) equipped with a krypton/argon mixed gas laser (Ion Laser Technology).

\section{In vivo and ex vivo imaging protocols mice and rabbits}

For mice and rabbits, the same imaging procedure was followed. In vivo SPECT imaging was performed at 3 hours after radiotracer administration using a dual-head microSPECT $\gamma$-camera combined with micro-CT (X-SPECT, Gamma Medica, Inc., Northridge, CA) under isoflurane anesthesia. SPECT images of the heart were acquired in a $64 \times 64$ matrix at 32 steps at 60 seconds per step with a $247 \mathrm{keV}$ photo peak of ${ }^{111}$ In with $15 \%$ windows using a medium energy parallel-hole collimator. In the animals also undergoing ${ }^{99 \mathrm{~m}}$ Tc-MIBI ${ }^{99 \mathrm{~m}}$ Tc-AA5, this was followed by imaging using the same protocol, at a 140 keV photo peak of ${ }^{99 \mathrm{~m}} \mathrm{Tc}$ with $15 \%$ windows using a low-energy, high-resolution parallelhole collimator. Gating was not performed. After SPECT imaging acquisition, a CT scan was acquired using an X-ray tube operating at $50 \mathrm{kVp}$ and $0.6 \mathrm{~mA}$. Images were acquired for 2.5 seconds per view for 256 views in $360^{\circ}$ rotation. After transferring to $256 \times 256$ 
matrix, the SPECT images and CT studies were fused. After imaging, anesthetized animals were terminated by heart explantation and ex vivo SPECT/CT excised heart was performed using the same protocols but at 45 seconds per step for SPECT. Thereafter, planar ${ }^{111}$ In-GSAO imaging of the hearts was performed for 15 minutes, followed by

${ }^{99 \mathrm{~m}}$ Tc-MIBI or ${ }^{99 \mathrm{~m}}$ Tc-AA5 planar imaging in GSAO-MIBI and GSAO-AA5 animals. After ex vivo imaging, rabbit hearts were cut into 4 short axis slices and further divided into 31-32 pieces. Mice hearts were cut in 3 short-axis slices. All sections were weighed, and $y$ counted in an automatic well-type $\gamma$-counter (Perkin Elmer Wallac Inc., Gaithersburg, $\mathrm{MD})$ for calculation of the percent total injected dose per tissue weight (\%ID/g) uptake. Tissue samples of the main organs were used for calculation of the \%ID/g uptake to evaluate the bio distribution. To correct for the radioactive decay and permit calculation of the concentration of radioactivity as a fraction of the administered dose, aliquots of the injected dose were counted simultaneously. For evaluation of infarct ${ }^{111}$ In-GSAO uptake in rabbits, the section with the highest ${ }^{111}$ In-GSAO uptake in the infarct area was used of each animal, and for evaluation of remote ${ }^{111}$ In-GSAO uptake, the section with the lowest ${ }^{111}$ In-GSAO uptake in the remote area was selected. In all mice, the apical slice was used for evaluation of infarct uptake, the middle slice for uptake in the border zone and the basal slice for remote uptake.

\section{Pharmacokinetic studies}

The pharmacokinetic parameters were derived using the KINFIT module of the MW/PHARM computer program package (Version 3.60, MediWare, Groningen, The Netherlands). ${ }^{42}$ The data, consisting of the blood concentration of ${ }^{111}$ In radioactivity or GSAO versus time, were analyzed by non-linear regression analysis using a least-squares weighted simplex algorithm, with data weighted with the reciprocal of the observed value.

\section{Histological, immunohistochemical evaluation}

After $\gamma$-counting of ${ }^{111}$ In-GSAO uptake, myocardial pieces of seven rabbits were processed for histopathologic characterization. Of each animal, two pieces from each region (infarct, border zone, remote) were selected.

The myocardial pieces were fixed overnight $4 \%$ paraformaldehyde in PBS $(\mathrm{pH} 7.4$ at $4^{\circ} \mathrm{C}$ ), and stored in PBS with $0.02 \%$ sodium azide at $4^{\circ} \mathrm{C}$ until used. The specimens were further processed by dehydration in a graded series of ethanol for paraffin-embedding. The blocks were cut in 5- $\mu \mathrm{m}$ thick sections, floated on a water bath containing deionized water $\left(43^{\circ} \mathrm{C}\right)$, and transferred to vectabond (Vector Laboratories Burlingame, California) reagent-treated slides (Vector SP-1800, Vector Laboratories), dried overnight, and stored until ready for use. Sections were deparaffinised by heating ( 25 minutes at $56^{\circ} \mathrm{C}$ ) and dehydration using xylene and graded series of ethanol. Tissue sections were stained 
with standard haematoxylin \& eosin and Masson's trichrome staining. For immunohistochemical characterization, adjacent sections were incubated with primary antibodies against Caspase-3. After washing with PBS, sections were incubated with a biotinylated secondary antibody. The presence of apoptotic cells was further evaluated using TUNEL staining as described previously. Briefly: exposed DNA fragments were labeled with biotinylated nucleotides (dNTPs) and TdT for $1 \mathrm{~h}$ at $37^{\circ} \mathrm{C}$ after blocking of endogenous perosidase activity using $0.3 \%$ hydrogen peroxide and incubation with proteinase $\mathrm{K}^{41}$ For color reactions, sections were incubated with diaminobenzidine. For the assessment of the immunopositive area, stained tissue sections were observed under appropriate magnification (Carl Zeiss, Thornwood, New York), and the images were captured with a high-resolution digital camera (Axiocam, 1,300 × 1,030 pixels, Carl Zeiss) using Axiovision 3.1 software. Digital images were analyzed using Image-Pro Plus 5.0 (Media Cybernetics, Bethesda, Maryland).

\section{Statistical analysis}

All results are presented as the mean \pm SD. In most cases, the data did not meet the assumptions of parametric tests. For consistency, non-parametric tests were used for all comparisons. Related samples were compared using Wilcoxon Signed Rank test (two groups) or Friedman's Two Way ANOVA followed by post-hoc Wilcoxon Signed-Rank tests and Bonferroni correction for pair-wise significance (more than two groups). Unrelated samples were compared using Mann Whitney $U$ tests (two groups) or KruskalWallis ANOVA followed by Mann-Whitney $U$ tests and Bonferroni Correction for pairwise significance (more than two groups). When the Kruskal-Wallis test revealed no significant differences among groups, no post-hoc tests were performed and P=NS was reported. Bonferroni correction was also applied to results of multiple Wilcoxon signed rank tests regarding the uptake of radiotracers in infarct vs. remote areas in rabbits (fig 3c). To assess the correlation between ${ }^{111} \mathrm{In}$-GSAO uptake and ${ }^{99 \mathrm{~m}} \mathrm{Tc}$-AA5 uptake, ${ }^{99 \mathrm{~m}} \mathrm{Tc}$ MIBI uptake and histologic findings (caspase-3 and TUNEL), Spearman's $\rho$ s were calculated. $P$ values of $<0.05$ were considered statistically significant.

\section{Acknowledgements}

This research was supported by a research grant from the International Research Fund for Subsidy of Kyusyu University School of Medicine Alumni and the Banyu Fellowship Program sponsored by Banyu Life Science Foundation International to N. Tahara, and research grants from the foundation "De Drie Lichten" and the Dutch Heart Association (Dr. E. Dekker student Grant) to H. de Haas. DTPA-GSAO, DTPA-GSCA, Cy5.5-GSAO and Cy5.5-GSCA were supplied by Covidien Imaging Solutions. We thank H.H. Boersma for performing blood clearance analysis. For excellent statistical support we thank E. Bagiella, supported by National Institutes of Health Grant UL1TR000067. 


\section{Author contributions}

J.N. conceived the study; R.P. developed and produced the targeting agent; M.D. developed the radiolabeling protocols; C.R. conceived the mouse fluorescence experiments; H.Z. conducted the mouse fluorescence experiments; H.Z. and A.P. conducted the mouse imaging experiments; N.T., A.P., T.Y. and T.S. conducted rabbit experiments; J.Z. conducted and analyzed the immunohistochemistry; N.T., H.H., A.P., J.N. performed data analysis; N.T. and H.H. performed statistical analysis; N.T., H.H., and J.N. prepared figures; N.T., H.H. and J.N. wrote the manuscript; J.N. funded the project; HZ, AP, RP, TY, JZ, $\mathrm{TI}, \mathrm{RS}, \mathrm{MD}, \mathrm{TS}, \mathrm{AK}, \mathrm{CR}, \mathrm{NN}, \mathrm{VF}$ helped in manuscript writing and editing.

\section{Additional Information}

Accessions Codes: NA

Supplementary Information: For additional data regarding biodistribution and blood clearance please refer to supplementary Fig. 1.

Competing Financial Interests: Dr. Dyszlewski is an employee of Covidien Imaging Solutions, Hazelwood, Missouri. Dr. Panduranghi was an employee of Covidien when the project was being conducted. The other authors claim no conflict of interest. 


\section{References}

1 Konstantinidis, K., Whelan, R. S. \& Kitsis, R. N. Mechanisms of cell death in heart disease. Arterioscler Thromb Vasc Biol 32, 1552-1562 (2012).

2 Nikoletopoulou, V., Markaki, M., Palikaras, K. \& Tavernarakis, N. Crosstalk between apoptosis, necrosis and autophagy. Biochim Biophys Acta 1833, 3448-3459 (2013).

3 Nicotera, P. \& Melino, G. Regulation of the apoptosis-necrosis switch. Oncogene 23, 2757-2765 (2004).

4 Boersma, H. H. et al. Past, present, and future of annexin A5: from protein discovery to clinical applications. J Nucl Med 46, 2035-2050 (2005).

5 Hofstra, L. et al. Visualisation of cell death in vivo in patients with acute myocardial infarction. Lancet 356, 209-212 (2000).

6 Thimister, P. W. et al. In vivo detection of cell death in the area at risk in acute myocardial infarction. $J$ Nucl Med 44, 391-396 (2003).

$7 \quad$ Narula, J. et al. Annexin- $\mathrm{V}$ imaging for noninvasive detection of cardiac allograft rejection. Nat Med 7, 1347-1352 (2001).

8 Kietselaer, B. L. et al. Noninvasive detection of programmed cell loss with 99mTc-labeled annexin A5 in heart failure. J Nucl Med 48, 562-567 (2007).

9 Khaw, B. A. \& Narula, J. Non-invasive detection of myocyte necrosis in myocarditis and dilated cardiomyopathy with radiolabelled antimyosin. Eur Heart J 16 Suppl O, 119-123 (1995).

10 Corbett, J. R. et al. 99mTc-pyrophosphate imaging in patients with acute myocardial infarction: comparison of planar imaging with single-photon tomography with and without blood pool overlay. Circulation 69, 1120-1128 (1984).

11 Mariani, G. et al. Detection of acute myocardial infarction by $99 \mathrm{mTc}$-labeled D-glucaric acid imaging in patients with acute chest pain. J Nucl Med 40, 1832-1839 (1999).

12 Narula, J. et al. Antimyosin uptake and myofibrillarlysis in dilated cardiomyopathy. J Nucl Cardiol 2, 470 477 (1995).

13 Park, D. et al. Noninvasive imaging of cell death using an Hsp90 ligand. J Am Chem Soc 133, 2832-2835 (2011).

14 Park, D. et al. Optical imaging of treatment-related tumor cell death using a heat shock protein-90 alkylator. Mol Pharm 10, 3882-3891 (2013).

15 Xie, B. W. et al. Optical imaging of cell death in traumatic brain injury using a heat shock protein-90 alkylator. Cell death \& disease 4, e473 (2013).

16 Donoghue, N., Yam, P. T., Jiang, X. M. \& Hogg, P. J. Presence of closely spaced protein thiols on the surface of mammalian cells. Protein Sci 9, 2436-2445 (2000).

17 Dilda, P. J., Ramsay, E. E., Corti, A., Pompella, A. \& Hogg, P. J. Metabolism of the tumor angiogenesis inhibitor 4-(N-(S-Glutathionylacetyl)amino)phenylarsonous acid. J Biol Chem 283, 35428-35434 (2008).

18 Goetz, M. P., Toft, D. O., Ames, M. M. \& Erlichman, C. The Hsp90 chaperone complex as a novel target for cancer therapy. Ann Oncol 14, 1169-1176 (2003).

19 Scarabelli, T. M. et al. Clinical implications of apoptosis in ischemic myocardium. Curr Probl Cardiol 31, 181-264 (2006).

20 Fliss, H. \& Gattinger, D. Apoptosis in ischemic and reperfused rat myocardium. Circ Res 79, 949-956 (1996).

21 Freude, B. et al. Apoptosis is initiated by myocardial ischemia and executed during reperfusion. J Mol Cell Cardiol 32, 197-208 (2000).

22 Gottlieb, R. A., Burleson, K. O., Kloner, R. A., Babior, B. M. \& Engler, R. L. Reperfusion injury induces apoptosis in rabbit cardiomyocytes. J Clin Invest 94, 1621-1628 (1994).

23 Narula, J. \& Strauss, H. W. Invited commentary: P.S.* I love you: implications of phosphatidyl serine (PS) reversal in acute ischemic syndromes. J Nucl Med 44, 397-399 (2003).

24 Kenis, $\mathrm{H}$. et al. Annexin A5 uptake in ischemic myocardium: demonstration of reversible phosphatidylserine externalization and feasibility of radionuclide imaging. J Nucl Med 51, 259-267 (2010). 


\section{CHAPTER 8}

25 Li, C. \& Jackson, R. M. Reactive species mechanisms of cellular hypoxia-reoxygenation injury. Am J Physiol Cell Physiol 282, C227-241 (2002).

26 Orrenius, S., Zhivotovsky, B. \& Nicotera, P. Regulation of cell death: the calcium-apoptosis link. Nat Rev Mol Cell Biol 4, 552-565 (2003).

27 Halestrap, A. P. Calcium, mitochondria and reperfusion injury: a pore way to die. Biochem Soc Trans 34, 232-237 (2006).

28 Halestrap, A. Biochemistry: a pore way to die. Nature 434, 578-579 (2005).

29 Halestrap, A. P., Gillespie, J. P., O'Toole, A. \& Doran, E. Mitochondria and cell death: a pore way to die? Symp Soc Exp Biol 52, 65-80 (2000).

30 Arbustini, E. \& Narula, J. Cyclosporin a in reperfusion injury: not opening to cell death knocking at the door? Ann Thorac Surg 89, 1349-1351 (2010).

31 Yue, T. L. et al. Possible involvement of stress-activated protein kinase signaling pathway and Fas receptor expression in prevention of ischemia/reperfusion-induced cardiomyocyte apoptosis by carvedilol. Circ Res 82, 166-174 (1998).

32 Condorelli, G. et al. Increased cardiomyocyte apoptosis and changes in proapoptotic and antiapoptotic genes bax and bcl-2 during left ventricular adaptations to chronic pressure overload in the rat. Circulation 99, 3071-3078 (1999).

33 Li, Z., Bing, O. H., Long, X., Robinson, K. G. \& Lakatta, E. G. Increased cardiomyocyte apoptosis during the transition to heart failure in the spontaneously hypertensive rat. Am J Physiol 272, H2313-2319 (1997).

34 Narula, J., Haider, N., Arbustini, E. \& Chandrashekhar, Y. Mechanisms of disease: apoptosis in heart failure--seeing hope in death. Nat Clin Pract Cardiovasc Med 3, 681-688 (2006).

35 De Saint-Hubert, M., Prinsen, K., Mortelmans, L., Verbruggen, A. \& Mottaghy, F. M. Molecular imaging of cell death. Methods (San Diego, Calif.) 48, 178-187 (2009).

36 Narula, J., Petrov, A., Pak, K. Y., Lister, B. C. \& Khaw, B. A. Very early noninvasive detection of acute experimental nonreperfused myocardial infarction with 99mTc-labeled glucarate. Circulation 95, 15771584 (1997).

37 Perugini, E. et al. Non-invasive evaluation of the myocardial substrate of cardiac amyloidosis by gadolinium cardiac magnetic resonance. Heart 92, 343-349 (2006).

38 Shimada, T. et al. Diagnosis of cardiac sarcoidosis and evaluation of the effects of steroid therapy by gadolinium-DTPA-enhanced magnetic resonance imaging. Am J Med 110, 520-527 (2001).

39 de Haas, H. J., Arbustini, E., Fuster, V., Kramer, C. M. \& Narula, J. Molecular imaging of the cardiac extracellular matrix. Circ Res 114, 903-915 (2014).

40 van den Borne, S. W. et al. Molecular imaging of interstitial alterations in remodeling myocardium after myocardial infarction. J Am Coll Cardiol 52, 2017-2028 (2008).

41 Kolodgie, F. D. et al. Targeting of apoptotic macrophages and experimental atheroma with radiolabeled annexin V: a technique with potential for noninvasive imaging of vulnerable plaque. Circulation 108, 3134-3139 (2003).

42 Proost, J. H. \& Meijer, D. K. MW/Pharm, an integrated software package for drug dosage regimen calculation and therapeutic drug monitoring. Computers in biology and medicine 22, 155-163 (1992). 
CHAPTER 9

Discussion 

Myocardial infarction (MI) remains a leading cause of morbidity and mortality worldwide. Myocardial infarction occurs when myocardial ischemia, a diminished blood supply to the heart, exceeds a critical threshold and overwhelms myocardial cellular repair mechanisms designed to maintain normal operating function and homeostasis. Ischemia at this critical threshold level for an extended period results in irreversible myocardial cell damage or death (1).

Cell death is often divided into two distinctive processes, apoptosis and necrosis; however, there is increasing evidence for additional pathways such as autophagy, mitotic catastrophe, and senescence. Apoptosis is characterized by classical morphology changes such as cytoplasm shrinkage, cell detachment, chromatin condensation, nuclear fragmentation and the formation of apoptotic bodies. In contrast, necrotic cells exhibit increased cytoplasmic vacuolation, organelle degeneration, condensation of chromatin into irregular patches, and an increase in cell volume that results in irreversible rupture of the plasma membrane.

The myocardium reacts to an ischemic challenge with a complex series of changes in cellular and extracellular components, characterized at the tissue level by altered wall structure, chamber geometry, and pump function, a process termed left ventricular remodelling (2-4). Furthermore, the lack of oxygen supply induces necrosis in the cardiac myocytes, which stimulates the complement cascade and initiates an inflammatory response (5). The inflammatory component is primarily composed of neutrophil and macrophage infiltration. In particular, the macrophage is a primary responder cell type that is involved in the regulation of post-MI wound healing at multiple levels. Indeed, following $\mathrm{Ml}$ circulating blood monocytes respond to chemotactic factors, migrate into the infarcted myocardium, and differentiate into macrophages. At the injury site, macrophages remove necrotic cardiac myocytes and apoptotic neutrophils; secrete cytokines, chemokines, and growth factors (6) and modulate phases of the angiogenic response (7). Moreover, the timing and rate of macrophage infiltration is regulated by a wide range of cytokines and chemokines (8) and plasminogen activation appears to be a clinical link between the activation of macrophages and collagen production in the heart in response to injury (9). In addition, as demonstrated in animal models, monocytes/macrophages dominate the cellular infiltrate for the first 2 weeks after $\mathrm{MI}$ and participate in infarct wound healing (10-14). According to recent studies, different subsets of macrophages are responsible for these different activities; during the early inflammatory phase (phase 1), proinflammatory macrophages dominate the injury site and phagocytose apoptotic/necrotic myocytes and other debris, whereas during inflammation resolution (phase 2 ), the dominant subsets are the reparative macrophages, which propagate infarct repair $(15,16)$. The duration and extent of the early inflammatory phase have major implications on infarct size and ventricle remodelling (17). Finally, impaired monocyte/macrophage function appears to be an unrecognized new pathophysiological mechanism for left ventricular thrombus development after MI (18). 
A significant number of $\mathrm{Ml}$ patients (3-40\%, depending on the study) will undergo adverse remodeling of the left ventricle (LV), leading to LV dilation and compromised LV function that drives the constellation of signs and symptoms indicative of congestive heart failure (CHF)(19). Hence MI predisposes patients to CHF, a significant long-term complication and leading cause of mortality in post-MI patients (3). Identifying vulnerable patients early in the pathogenesis, therefore, will allow better risk stratification postMI (20-22). Since the macrophage regulates multiple aspects of the post-MI wound healing response, it represents a likely candidate for investigation and intervention. Nonetheless, while many studies correlate macrophage infiltration with changes in LV remodeling parameters, direct causal links between macrophages and many of the above-mentioned processes remains to be experimentally established and the effect of monocyte/macrophage recruitment after MI remains not fully defined.

Therefore one of the main aim of our research was to study the role of macrophages during $\mathrm{Ml}$ and to establish the correlation between this role and the complex processes leading to collagen deposition, fibrosis, remodeling which end with ireeversible cardiac failure.

Molecular imaging is a highly active research discipline that has seen tremendous growth over the past decade. It has broadened our understanding of oncologic, neurologic, and cardiovascular diseases by providing new insights into the in vivo biology of disease progression and therapeutic interventions. As it allows for the longitudinal evaluation of biological processes, it is ideally suited for monitoring treatment response. In $\mathrm{MI}$ and heart failure three biological features represents attractive targets for molecular imaging: 1) apoptosis, a form of programmed cell death which has been shown to play a key role in the process of gradual loss of pump function in heart failure, 2) The reninangiotensin pathway which plays a pivotal role in the development of post-MI heart failure and 3) The biological processes of angiogenesis and fibrogenesis which are considered key processes in myocardial scar formation and post-MI ventricular remodeling.

\section{Main findings}

\section{New insights into the role of macrophages after myocardial infarction}

Despite previous demonstration that macrophages may contribute to myocardial dysfunction by producing inflammatory and profibrotic mediators (23) our experience suggests that the depletion of macrophages results in accelerated development of endstage cardiomyopathy and CHF. In addition, our research poses an important question on the effective role of the inflammation on the entire process. In fact, while the importance of the collagen framework in healing from myocardial injury has been widely demonstrated (24), and reduction of collagen deposition by neurohumoral antagonists is a well known mechanism to prevent ventricular remodelling (25), on the other hand it is 
undoubtedly clear from our findings that lack of collagen deposit in the infarct zone allows accelerated ventricular remodeling. Indeed, we observed that after depletion of macrophages, the complete abolition of the fibrotic process including fibroblast and collagen deposition was observed. Therefore, it is reasonable to think that the complete abolition of fibrosis in absence of macrophages is a negative factor leading to CHF. This is in accordance with previous reports (26) and confirms the findings of published studies showing prevention of LV remodelling following intra-myocardial injection of collagen (27).

Macrophages can directly influence disease progression by altering the balance between matrix synthesis and degradation through protease and cytokine secretion $(28,29)$. Duffield et al. (30) demonstrated in transgenic mice, in which macrophage depletion is temporally regulated, that macrophage depletion during active remodelling reduced matrix deposition, whereas their removal during resolution phase exacerbated matrix deposition. However, in cardiac disease models, reduced monocyte/macrophage infiltration is accompanied by a substantial reduction in fibrosis (31). Interaction between mineralcorticoid receptors (MR) signalling and macrophages appear to be important in inflammatory conditions in the heart although the role of macrophage MR signalling in mediating these responses is unknown. Rickard et al. (31) demonstrated that selective deletion of MR from macrophages protected against mineralocorticoidmediated cardiac fibrosis, despite normal macrophage recruitment. In the macrophage MR-null mice, the reduced cardiac damage appears to be due to altered macrophage function following loss of MR signalling from these cells. In contrast, the sustained macrophage recruitment is thought to be consequence of MR signalling in other cell types in the cardiovascular system following mineralocorticoid/salt treatment. This is not in contrast with our findings since we can postulate that the development of cardiomyopaty secondary to macrophage depletion can be due to over expression of MR on the surface of these cells. However, this aspect was not explored and deserves further investigation. It will be object of ongoing studies.

A variety of cytokines have been implicated in cardiac remodeling as well as in healing such as interleukin (IL)-1 $\beta$ and tumor necrosis factor alpha (TNF- $\alpha$ ). The expression of TNF can have multiple effects yet extracellular matrix remodeling and it seems to be of major importance (32). Moreover, mice with over expression of TNF develop LV dilatation and changes in collagen content prevented by a matrix metalloproteinase (MMP) inhibitor (33), further highlighting a potential importance of excessive activation of proinflammatory cytokines on matrix remodeling and wound healing in the heart. Nonetheless, Bilate and co-workers (34) showed that TNF $\alpha$ /LT-alpha blockade with etanercept enhances left ventricular dysfunction in trypanosoma cruzi-induced chronic cardiomyopathy. This is in accordance with our findings since TNF- $\alpha$ is produced primarily by macrophages and TNF- $\alpha$ signaling may be cardioprotective, preventing cardiomyocite apoptosis after myocardial infarction (35). Interestingly, macrophage-deleted hearts showed abundant compensatory T-lymphocyte infiltration, mainly composed by Th1 and Th17. 
We can hypotize that deleterious effects of CD4 T-cell infiltration plays an important role and contributes to deterioration of cardiac function after sustained depletion of macrophages.

Another interesting finding of our research is that functional deterioration of the heart was more pronounced when macrophages were present in the initial two weeks after MI whereas an improvement was observed if they were depleted early suggesting that a reduction in macrophage infiltration early after $\mathrm{Ml}$ might be beneficial. It is presumable that, although macrophages are necessary to scavenge the necrotic debris from the infarct size (36), the accompanying cytokine activity may delay the healing process (37).

We also explored the transdifferentiation from macrophage to fibroblast during MI. We demonstrated that macrophages derived from $\mathrm{CD} 14^{+}$human monocytes gradually transformed into cells of fibroblast-like phenotype. These cell expressed all fibroblast markers most of which increased during the conversion process. Furthermore, and more importantly, these cells were able to secrete collagen I into the culture medium. This was confirmed by our experimental model of $\mathrm{Ml}$ in transgenic mice marked with yellow florescent protein (YFP): YFP-expressing-macrophages were easily identified in the tissue sections and showed fibroblast markers indicating a transaction from macrophage to fibroblast-like cell.

We believe that a pro-fibrotic environment might induce this transdifferentiation that results in fibroblasts which, differently from "normal fibroblasts" have less "competence" in maintaining the collagen homeostasis, resulting in uncontrolled collagen and extracellular matrix deposition responsible of disease progression. Mechanical tension, TGF- $\beta 1$ and fibronectin induce cardiac fibroblasts trans-differentiate into myofibroblasts, predominant cell types that synthesize the extracellular matrix $(38,39)$. Furthermore, cathepsins (Cat K-L-S) are potent proteases which are able to degrade collagen and fibronectin (40) and, in a recent study Chen et al (41) demonstrated that cathepsin S regulates myofibroblast trans-differentiation and myocardium extracellular matrix protein differentiation. Interestingly, proinflammatory macrophages and T-cells express high levels of cathepsins $(42,43)$ therefore cathepsins apart from mediating extracellular matrix degradation and participating importantly in post-MI left ventricular remodeling, might also have a role in transdifferentiation from macrophage to fibroblast during $\mathrm{MI}$. This finding might have important repercussions on new anti remodeling therapies with cathepsin-inhibitors. However, macrophage to fibroblast transdifferentiation is a major new frontier in cardiovascular biology. Successful manipulation of this pathway is likely to have a positive impact on myocardial fibrosis, adverse myocardial remodeling and heart failure. 


\section{From cellular biology to molecular imaging}

The balance between proliferation and programmed cell death-apoptosis-is essential for multicellular organisms which use apoptosis to regulate and maintain the number and type of their cells during embryogenesis, growth and homeostasis (44). Increased cell proliferation or enhanced cell loss can be caused by dysregulated apoptosis and are observed in various diseases such as myocardial infarction. Therefore, non-invasive imaging of apoptosis is of great clinical interest as patients would clearly benefit from the diagnosis of cell loss post infarction. Several biochemical transformations occur in apoptotic cells offering different biological targets for the development of specific molecular biomarkers of apoptosis. Key steps that occur during apoptosis have already been evaluated; among these are the externalisation of phospholipid phosphatidylserine to the outer leaflet of the cell membrane, which can be visualized by labeled annexin-A5 (AA5) and the activation of caspases, especially effector caspase-3, which can be addressed by labeled enzyme substrates or synthetic caspase inhibitors.

We demonstrated that ischemia-induced apoptosis in cardiomyocytes is interrupted by reperfusion such that cardiomyocytes survive. Therefore, our data confirm recovery from apoptosis in vivo, despite activation of caspase 3. Reversible phosphatidylserine exposure not associated with the execution of apoptosis was previously demonstrated in myocitic cell lines and B-cell lymphoma cells (45-47).

Another interesting finding of our research is that the binding of AA5 to externalized phosphatidylserine has a "protective" function avoiding the complex being recognized by phagocytes which distinguish the externalized phosphatidylserine as signal for the engulfment of apoptotic cells (48). Hence, AA5 my serve as an acute-phase reactant which may save cells susceptible to engulfment and we can assume that exogenously administered AA5 may accelerate the internalization of externalized phosphatidylserine. Therefore, our data suggest a novel model of phosphatidylserine exposure and internalization in which the exposure is continuously driven by caspase activation whereas internalization is mediated by AA5-aided formation of the endocytic vesicle (49). The reversible phosphatidylserine exposure confirmed to be an attractive target for molecular imaging. The administration of radiolabel AA5 allows to get images even after brief ischemia and with a no extensive phosphatidylserine exposure since the complex AA5 phosphatidylserine is internalized and may concentrate radiolabeled AA5 in the ischemic cells. The transient exposure of phosphatidylserine and the persistence of phosphatidylserine exposure at least 6 hours after ischemic stress may be exploited in the clinical practice, for instance for retrospective diagnosis of cardiac chest pain.

Heat shock protein 90 (HSP90) is an ATP-dependent molecular chaperone responsible for protein quality control in cells. HSP9O has been shown to be overexpressed for instance in many human cancers. This has prompted extensive research on HSP9O inhibitors as novel anticancer agents and, more recently, the development of molecular probes for imaging HSP90 expression in vivo (50). In addition, the HSP90 is a cytoprotec- 
tive protein chaperone that participates in mitochondrial import of a number of proteins and it has been demonstrated that HSP90-mediated mitochondrial import of Protein kinase $\mathrm{C}$ epsilon $\left(\mathrm{PKC}_{\varepsilon}\right)$ plays an important role in the protection of the myocardium from ischemia-reperfusion injury in an animal model of heart ischemia since pharmacological inhibition of HSP90 prevented ischemia-reperfusion -induced interaction between $\mathrm{PKC}_{\varepsilon}$ and the translocase of the outer membrane (Tom20), reduced mitochondrial import of $\mathrm{PKC}_{\varepsilon}$, and increased necrotic cell death by $\approx 70 \%$ (51 ). Moreover, inducible nitric oxide synthase (iNOS) is an important key in the process of myocardial infarction, heart failure, and cardiac preconditioning (52). The expression of iNOS is mainly induced by inflammatory substances such as endotoxins and cytokines. Previous studies showed that HSP9O associates with iNOS and this association enhances iNOS activity. HSP9O is required for iNOS gene transactivation and intervening on it may represent a novel approach to modulate iNOS expression in myocardial infarction and cardiac preconditioning (53).

We exploited the capacity of radiolabeled trivalent arsenical peptide, 4-(N-(Sglutathionylacetyl) amino) phenylarsonous acid (GSAO) to target intracellular dithiol molecules like HSP90. This has been already employed for non-invasive identification of tumor cell death (54). HSP90 contains a pair of highly conserved cysteines (Cys719 and Cys 720) that can be selectively cross-linked by the GSAO probe. The high abundance of HSP90 makes it an attractive target for sensitive molecular imaging; however, it appears that the cell membrane must be partially compromised for GSAO entry. It is not precisely known when, and by how much, plasma membrane permeability changes as a cell progresses from early to late stage apoptosis. The plasma membrane of an apoptotic cell has high integrity and is permeable to only a select group of small molecules, including nucleotides and cyanine dyes (due in part to caspase-mediated activation of the membrane channel protein, pannexin (55). In contrast, the plasma membrane of a necrotic cell is completely disrupted with free access of small and large molecules to the cell interior $(56,57)$. We demonstrated the feasibility of cell death imaging in AMI using GSAO and the specificity of the radiotracer was confirmed by the localization of GSAO in the infracted zone. Our fluorescent experiments showed that GSAO accumulated intracellularly and this only occurred in AA5 positive cells supporting the notion that necrosis followed apoptotic signalling in the setting of acute myocardial infarction and it may play a dominant role.

This confirms also that often there is a crosstalk between multiple death processes that are occurring simultaneously and it must be considered that in cell death different death processes can induce the same change in one biomarkers. For example, phosphatidylserine exposure, reactive oxygen species (ROS) generation, and lysosomal membrane permeabilization (LMP) are biomarkers associated with both apoptosis and necrosis $(58-60)$.

It is well known that upregulation of the renin-angiotensin system (RAS) after myocardial infarction contributes to the process of the cardiac remodeling and hence the evolution of heart failure (61-64). Using ligands for the primary RAS mediator in myocar- 
dium, the angiotensin II type 1 receptor (AT1R), studies showed that regional AT1R upregulation can be visualized non-invasively in rodents after myocardial infarction $(65,66)$. But interspecies differences of RAS have been reported $(67,68)$, and the usefulness of cardiac AT1R imaging in large mammals and humans remains to be demonstrated although recent data confirm that targeted molecular imaging of cardiac AT1R expression is feasible using clinical PET/CT technology and that, in the future, this technique may provide unique insights into regional myocardial RAS activation in cardiac disease (69). Furthermore, it has been recently reported that studies on the use of a novel arginineglutamate-aspartate (RGD) peptide, technetium-99m-labeled Cy5.5-RGD-imaging peptide (CRIP) can be used for molecular imaging and to evaluate changes in collagen synthesis in mice that were treated with angiotensin and/or aldosterone antagonists, either alone and in combination. CRIP is a cyclic peptide that was originally developed to image integrins such as avB3/5, which are up regulated during angiogenesis (70). Subsequently, CRIP was shown to bind to integrins expressed in activated myofibroblasts as well as to correlate with new collagen synthesis within the infarct zone (71). We evaluated the effect of an angiotensin-converting enzyme inhibitor (ACE-I), an angiotensin receptor blocker (ARB), and a selective aldosterone receptor antagonist (SARA), individually and in combination, on infarct healing by using $99 \mathrm{mTC}$-CRIP imaging. The quantitative CRIP percent injected dose per gram uptake was greatest in the infarct area of untreated control mice and decreased significantly in animals treated with 1 or 2 agents. The addition of 3 agents demonstrated a further, although not statistically significant, reduction in tracer uptake. Moreover, the decrease in echocardiographic left ventricular function, strain and rotation parameters, as well as histologically verified deposition of thin collagen fibers, was significantly reduced in treatment groups and correlated with CRIP uptake. This demonstrates the efficacy of antiangiotensin and antialdosterone intervention on interstitial alterations and that the radiotracer uptake correlated closely with parameters of depressed myocardial mechanics, the quantitative prevalence of myofibroblasts, and thin fiber collagen deposition, suggesting that combined treatment with neurohumoral antagonists results in decreased new collagen production. Data obtained are in accordance with information provided by the estimation of collagen synthesis and fragmentation markers in the serum (72-74).

However, there are 2 potential limitations of this novel imaging approach that warrant further discussion. First, the in vivo data suggest that CRIP binds predominately to avB3/5 integrins on activated myofibroblasts. It bears emphasis that avB3/5 integrins are expressed on a variety of cell types, including macrophages, intimal and medial smooth cells, endothelial cells of microvessels, and platelets. Although CRIP binding was largely confined to the infarct zone this finding may have occurred because there was a predominance of myofibroblasts at the time radiolabeled CRIP was administered. Thus, the signal-to-noise ratio of this novel molecular imaging agent may become problematic in the clinical setting, wherein the timing of infarction is not as well known and/or patients are being administered anti-inflammatory agents (e.g., aspirin and statins) that decrease 
inflammatory signaling, which might decrease the conversion of fibroblasts to myofibroblasts. Second, it is unclear from studies in the literature in which the authors used acute coronary artery occlusions whether the signal from radiolabeled CRIP is sufficient to image myocardial fibrosis in smaller infarcts (e.g., non-ST-segment infarcts) and/or myocardial fibrosis after reperfusion injury, wherein there is an influx of inflammatory cells that express avB3/5 integrins. Notwithstanding these potential therapeutic limitations, the initial use of CRIP appears promising and offers a potentially exciting new "looking glass" for monitoring the development and progression of myocardial fibrosis during cardiac remodeling.

\section{Conclusions}

Following $\mathrm{MI}$, circulating blood monocytes respond to chemotactic factors, migrate into the infarcted myocardium, and differentiate into macrophages. At the injury site, macrophages remove necrotic cardiac myocytes and apoptotic neutrophils; secrete cytokines, chemokines, and growth factors; and modulate phases of the angiogenic response. As such, the macrophage is a primary responder cell type that is involved in the regulation of post-MI wound healing at multiple levels. Our studies correlated macrophage infiltration with changes in LV remodeling parameters, confirming the exciting results already seen in the macrophage biology arena revealing avenues for basic science research, in regards to macrophage function in the post-MI setting specifically and the wound-healing environment. Inhibition studies using anti-inflammatory strategies have taught us the importance of discerning beneficial and detrimental roles for the macrophage post-MI. The ability to isolate these opposing functions could potentially be used to derive novel therapeutic strategies.

In addition, the ongoing efforts of a large international group of biomedical researchers are greatly expanding the collective understanding of cell death and its role in a range of diseases and therapeutic treatments. Recent research advances have greatly increased our molecular understanding of cell death and its role in a range of diseases and therapeutic treatments. Central to these ongoing research and clinical efforts is the need for imaging technologies that can locate and identify cell death in a wide array of in vitro and in vivo biomedical samples with varied spatiotemporal requirements. Useful biomarkers for dead and dying cells have been identified to develop molecular probes that target these biomarkers for optical, radionuclear, or magnetic resonance imaging. Our experiences confirm that high contrast image can be achieved within a specific window in time that will depend on the specifics of the cell death phenomenon under study. Our findings require further larger studies to be confirmed. 


\section{References}

1. Fishbein MC, Maclean D, Maroko PR. The histopathologic evolution $f$ myocardial infarction. Chest 1978;73:843-9.

2. Lindsey ML. MMP induction and inhibition in myocardial infarction. Heart Fail Rev 2004;9:7-19.

3. Opie LH, Commerford PJ, Gersh BJ, Pfeffer MA. Controversies in ventricular remodelling. Lancet 2006;367:356-67.

4. Pfeffer MA, Braunwald E. Ventricular remodeling after myocardial infarction. experimental observations and clinical implications.Circulation 1990;81:1161-72.

5. Rossen RD, Michael LH, Kagiyama A, et al. Mechanism of complement activation after coronary artery occlusion: evidence that myocardial ischemia in dogs causes release of constituents of myocardial subcellular origin that complex with human C1q in vivo. Circ Res 1988;62:572-84.

6. Lambert JM, Lopez EF, Lindsey ML. Macrophage roles following myocardial infarction. Int J Cardiol. 2008 Nov 12;130(2):147-58.

7. Ren G. Inflammatory mechanisms in myocardial infarction. Curr Drug Targets Inflamm Allergy 2003;2:242-56.

8. Frangogiannis NG, Smith CW, Entman ML. The inflammatory response in myocardial infarction. Cardiovasc Res 2002;53:31-47.

9. Minami E, Castellani C, Malchodi L, Deem J, Bertko K, Meznarich J, Dishmon M, Murry CE, StempienOtero A.The role of macrophage-derived urokinase plasminogen activator in myocardial infarct repair: urokinase attenuates ventricular remodeling. J Mol Cell Cardiol. 2010 Sep;49(3):516-24.

10. Weihrauch D, Zimmermann R, Arras M, Schaper J. Expression of extracellular matrix proteins and the role of fibroblasts and macrophages in repair processes in ischemic porcine myocardium. Cell Mol Biol Res. 1994; 40: 105-116. Medline

11. Sun Y, Weber KT. Cells expressing angiotensin II receptors in fibrous tissue of rat heart. Cardiovasc Res. 1996; 31: 518-525.

12. Yang F, Liu YH, Yang XP, Xu J, Kapke A, Carretero OA. Myocardial infarction and cardiac remodelling in mice. Exp Physiol. 2002; 87: 547-555.

13. Frangogiannis NG, Mendoza LH, Ren G, Akrivakis S, Jackson PL, Michael LH, Smith CW, Entman ML. MCSF expression is induced in healing myocardial infarcts and may regulate monocyte and endothelial cell phenotype. Am J Physiol Heart Circ Physiol. 2003; 285: H483-H492.

14. Dewald O, Ren G, Duerr GD, Zoerlein M, Klemm C, Gersch C, Tincey S, Michael LH, Entman ML, Frangogiannis NG. Of mice and dogs: species-specific differences in the inflammatory response following myocardial infarction. Am J Pathol. 2004; 164: 665-677.

15. Nahrendorf M, Swirski FK, Aikawa E, Stangenberg L, Wurdinger T, Figueiredo JL, Libby P, Weissleder R, Pittet MJ.The healing myocardium sequentially mobilizes two monocyte subsets with divergent and complementary functions. J Exp Med 2007; 204 (12):3037-3047.

16. Troidl C, Möllmann H, Nef H, Masseli F, Voss S, Szardien S, Willmer M, Rolf A, Rixe J, Troidl K, Kostin S, Hamm C, Elsässer A.Classically and alternatively activated macrophages contribute to tissue remodelling after myocardial infarction. J Cell Mol Med 2009; 13 (9B):3485-3496.

17. Henson PM. Dampening inflammation. Nat Immunol 2005; 6:1179-1181.

18. Frantz S, Hofmann U, Fraccarollo D, Schäfer A, Kranepuhl S, Hagedorn I, Nieswandt B, Nahrendorf M, Wagner H, Bayer B, Pachel C, Schön MP, Kneitz S, Bobinger T, Weidemann F, Ertl G, Bauersachs J. Monocytes/macrophages prevent healing defects and left ventricular thrombus formation after myocardial infarction. FASEB J. $2013 ; 27(3): 871-81$.

19. Solomon SD, Glynn RJ, Greaves S, Ajani U, Rouleau JL, Menapace F, Arnold JM, Hennekens C, Pfeffer MA. Recovery of ventricular function after myocardial infarction in the reperfusion era: the healing and early afterload reducing therapy study. Ann Intern Med 2001;134 (6) :451-8.

20. Anavekar NS, McMurray JJ, Velazquez EJ, Solomon SD, Kober L, Rouleau JL, White HD, Nordlander R, Maggioni A, Dickstein K, Zelenkofske S, Leimberger JD, Califf RM, Pfeffer MA.Relation between renal dysfunction and cardiovascular outcomes after myocardial infarction. N Engl J Med 2004;351(13):1285-95. 
21. Sanz G. Risk stratification in acute coronary syndromes: an unresolved issue. Rev Esp Cardiol 2007;60 Suppl 3:23-30.

22. Bhatheja R, Mukherjee D. Acute coronary syndromes: unstable angina/non-ST elevation myocardial infarction. Crit Care Clin 2007;23 (4):709-35.

23. Sharma UC, Pokharel S, van Brakel TJ, van Berlo JH, Cleutjens JP, Schroen B, André S, Crijns HJ, Gabius HJ, Maessen J, Pinto YM.Galectin-3 marks activated macrophages in failure-prone hypertrophied hearts and contributes to cardiac dysfunction. Circulation. 2004;110 (19) :3121-3128.

24. Frangogiannis NG, Smith CW, Entman ML. The inflammatory response in myocardial infarction. Cardiovasc Res. 2002 Jan;53(1):31-47.

25. van den Borne SW, Isobe S, Verjans JW, Petrov A, Lovhaug D, Li P, Zandbergen HR, Ni Y, Frederik P, Zhou J, Arbo B, Rogstad A, Cuthbertson A, Chettibi S, Reutelingsperger C, Blankesteijn WM, Smits JF, Daemen MJ, Zannad F, Vannan MA, Narula N, Pitt B, Hofstra L, Narula J.Molecular imaging of interstitial alterations in remodeling myocardium after myocardial infarction. J Am Coll Cardiol. 2008 ;52(24):2017-28.

26. van Amerongen MJ, Harmsen MC, van Rooijen N, Petersen AH, van Luyn MJ. Macrophage depletion impairs wound healing and increases left ventricular remodeling after myocardial injury in mice. Am J Pathol. 2007;170:818-829.

27. Dai W, Wold LE, Dow JS, Kloner RA. Thickening of the infarcted wall by collagen injection improves left ventricular function in rats: a novel approach to preserve cardiac function after myocardial infarction. J Am Coll Cardiol. 2005;46:714-719.

28. Lupher ML Jr, Gallatin WM. Regulation of fibrosis by the immune system. Advances in Immunology 2006; 89 245-288.

29. Wynn TA. Cellular and molecular mechanisms of fibrosis. Journal of Pathology 2008; 214 199-210.

30. Duffield JS, Forbes SJ, Constandinou CM, Clay S, Partolina M, Vuthoori S, Wu S, Lang R \& Iredale JP. Selective depletion of macrophages reveals distinct, opposing roles during liver injury and repair. Journal of Clinical Investigation 2005; 115 56-65.

31. Rickard AJ, Young MJ. Corticosteroid receptors, macrophages and cardiovascular disease. Mol Endocrinol .2009; 42: 449-459

32. Pagani F.D., Baker L.S., Hsi C., Knox M., Fink M.P., Visner M.S.Left ventricular systolic and diastolic dysfunction after infusion of tumor necrosis factor-alpha in conscious dogs. J Clin Invest 1992; 90:389-398.

33. Diwan A, Dibbs Z, Nemoto S, Defreitas G, Carabello BA, Sivasubramanian N, Wilson EM, Spinale FG, Mann DL.Targeted overexpression of noncleavable and secreted forms of tumor necrosis factor provokes disparate cardiac phenotypes. Circulation 109 (2):262-268.

34. Bilate AM, Salemi VM, Ramires FJ, de Brito T, Russo M, Fonseca SG, Faé KC, Martins DG, Silva AM, Mady C, Kalil J, Cunha-Neto E. TNF blockade aggravates experimental chronic Chagas disease cardiomyopathy. Microbes Infect. 2007;9:1104-1113.

35. Kurrelmeyer KM, Michael LH, Baumgarten G, Taffet GE, Peschon JJ, Sivasubramanian N, Entman ML, Mann DL. Endogenous tumor necrosis factor protects the adult cardiac myocyte against ischemicinduced apoptosis in a murine model of acute myocardial infarction. Proc Natl Acad Sci U S A. 2000;97(10):5456-61.

36. van den Borne SW, Diez J, Blankesteijn WM, Verjans J, Hofstra L, Narula J. Myocardial remodeling after infarction: the role of myofibroblasts. Nat Rev Cardiol 2010; 7(1): 30-7.

37. Arslan F, Smeets MB, Riem Vis PW, Karper JC, Quax PH, Bongartz LG, Peters JH, Hoefer IE, Doevendans PA, Pasterkamp G, de Kleijn DP. Lack of fibronectin-EDA promotes survival and prevents adverse remodeling and heart function deterioration after myocardial infarction. Circ Res 2011;108(5):582-592.

38. Serini G, Bochaton-Piallat ML, Ropraz P, Geinoz A, Borsi L, Zardi L, Gabbiani G.The fibronectin domain ED$A$ is crucial for myofibroblastic phenotype induction by transforming growth factor-beta1. J Cell Biol 1998;142(3):873-881.

39. Chapman HA, Riese RJ, Shi GP. Emerging roles for cysteine proteases in human biology. Annu Rev Physiol 1997;59:63-88.

40. Taleb S, Cancello R, Cle'ment K, Lacasa D. Cathepsin s promotes human preadipocyte differentiation: possible involvement of fibronectin degradation. Endocrinology 2006; 147:4950-4959. 
41. Chen H, Wang J, Xiang MX, Lin Y, He A, Jin CN, Guan J, Sukhova GK, Libby P, Wang JA, Shi GP.Cathepsin Smediated fibroblast trans-differentiation contributes to left ventricular remodelling after myocardial infarction. Cardiovasc Res. 2013;100(1):84-94.

42. ShiGP,Munger JS, Meara JP, Rich DH,Chapman HA. Molecular cloning and expression of human alveolar macrophage cathepsin S, an elastinolytic cysteine protease. J Biol Chem 1992;267:7258-7262.

43. Kitamoto S, Sukhova GK, Sun J, Yang M, Libby P, Love V, Duramad P, Sun C, Zhang Y, Yang X, Peters C, Shi GP. Cathepsin $L$ deficiency reduces diet-induced atherosclerosis in low-density lipoprotein receptorknockout mice. Circulation 2007;115 (15):2065-2075.

44. Faust A, Hermann S, Wagner S, Haufe G, Schober O, Schäfers M, Kopka K. Molecular imaging of apoptosis in vivo with scintigraphic and optical biomarkers--a status report. Anticancer Agents Med Chem. 2009 ;9(9):968-85.

45. van den Eijnde SM, van den Hoff MJ, Reutelingsperger CP, van Heerde WL, Henfling ME, Vermeij-Keers C, Schutte B, Borgers M, Ramaekers FC. Transient expression of phosphatidylserine at cell-cell contact areas is required for myotube formation. J Cell Sci. 2001;114(pt20):3631-3642.

46. Kaspar P, Dvorak M. Involvement of phosphatidylserine externalization in the down-regulation of c-myb expression in differentiating C2C12 cells. Differentiation. 2008;76:245-252.

47. Hammill AK, Uhr JW, Scheuermann RH. Annexin V staining due to loss of membrane asymmetry can be reversible and precede commitment to apoptotic death. Exp Cell Res. 1999;251:16-21.

48. Savill J, Fadok V. Corpse clearance defines the meaning of cell death. Nature.2000;407:784-788.

49. Kenis H, van Genderen H, Bennaghmouch A, Rinia HA, Frederik P, Narula J, Hofstra L, Reutelingsperger CP. Cell surface-expressed phosphatidylserine and annexin A5 open a novel portal of cell entry. J Biol Chem. 2004;279:52623-52629.

50. Wuest F, Bouvet V, Mai B, LaPointe P.Fluorine- and rhenium-containing geldanamycin derivatives as leads for the development of molecular probes for imaging Hsp90. Org Biomol Chem. 2012;10(33):672431.

51. Budas GR, Churchill EN, Disatnik MH, Sun L, Mochly-Rosen D.Mitochondrial import of PK Cepsilon is mediated by HSP90: a role in cardioprotection from ischaemia and reperfusion injury. Cardiovasc Res. $2010 ; 88(1): 83-92$.

52. Vladic N, Ge ZD, Leucker T, Brzezinska AK, Du JH, Shi Y, Warltier DC, Pratt PF Jr, Kersten JR.Decreased tetrahydrobiopterin and disrupted association of Hsp90 with eNOS by hyperglycemia impair myocardial ischemic preconditioning. Am J Physiol Heart Circ Physiol. 2011;301(5):H2130-9.

53. Luo S, Wang T, Chen LL, Xia Y. The Obligatory Role of Heat Shock Protein 90 in iNOS Induction in Postischemic Hearts. Circulation. 2009;120:S1060.

54. Park D, Don AS, Massamiri T, Karwa A, Warner B, MacDondald J, Hemenway C, Naik A, Kuan KT, Dilda PJ, Wong JW, Camphausen K, Chinen L, Dyszlewski M, Hogg PJ. Noninvasive Imaging of Cell Death using an Hsp90 Ligand. J Am Chem Soc. 2011;133:2832-2835.

55. Chekeni FB, Elliot MR, Sandilos JK, Walk SF, Kinchen JM, Lazarowski ER, Armstrong AJ, Penuela S, Laird DW, Salvesen GS, Isakson BE, Bayliss DA, Ravichandran KS. Pannexin 1 Channels Mediate "Find-Me" Signal Release and Membrane Permeability During Apoptosis. Nature. 2010;467:863-867.

56. Smith BA, Smith BD. Biomarkers and Molecular Probes for Cell Death Imaging and Targeted Therapeutics. Bioconjug Chem. 2012 ; 23(10): 1989-2006.

57. Maiuri MC, Zalckvar E, Kimchi A, Kroemer G. Self-Eating and Self-Killing: Crosstalk Between Autophagy and Apoptosis. Nat Rev Mol Cell Biol. 2007;8:741-752.

58. Krysko O, de Ridder L, Cornelissen M. Phosphatidylserine Exposure During Early Primary Necrosis (Oncosis) in JB6 Cells as Evidenced by Immunogold Labeling Technique. Apoptosis. 2004;9:495-500.

59. Festjens N, Vanden Berghe T, Vandenabeele P. Necrosis, a Well- Orchestrated Form of Cell Demise: Signaling Cascades, Important Mediators and Concomitant Immune Response. Biochim Biophys Acta. 2006;1757:1371-1378.

60. Galluzzi L, Maiuri MC, Vitale I, Zischka H, Castedo M, Zitvogel L, Kroemer G. Cell Death Modalities: Classification and Pathophysiological Implications. Cell Death Differ. 2007;12:1237-1266. 
61. Weber KT, Brilla CG. Pathological hypertrophy and cardiac interstitium. Fibrosis and reninangiotensinaldosterone system. Circulation 1991; 83:1849-65.

62. Weber KT, Anversa P, Armstrong PW. Remodeling and reparation of the cardiovascular system. J Am Coll Cardiol 1992;20:3-16.

63. Pitt B. Aldosterone blockade in patients with systolic left ventricular dysfunction. Circulation 2003;108: 1790-4.

64. Shirani J, Narula J, Eckelman WC, Narula N, Dilsizian V. Early imaging in heart failure: exploring novel molecular targets. J Nucl Cardiol 2007;14:100 -10.

65. Higuchi T, Fukushima K, Xia J, Mathews WB, Lautamäki R, Bravo PE, Javadi MS, Dannals RF, Szabo Z, Bengel FM. Radionuclide imaging of angiotensin II type 1 receptor upregulation after myocardial ischemiareperfusion injury. J Nucl Med 2010;51(12):1956-61.

66. Verjans JW, Lovhaug D, Narula N, Petrov AD, Indrevoll B, Bjurgert E, Krasieva TB, Petersen LB, Kindberg GM, Solbakken M, Cuthbertson A, Vannan MA, Reutelingsperger CP, Tromberg BJ, Hofstra L, Narula J. Noninvasive imaging of angiotensin receptors after myocardial infarction. J Am Coll Cardiol Img 2008;1(3):354-62.

67. Gallagher AM, Bahnson TD, Yu H, Kim NN, Printz MP. Species variability in angiotensin receptor expression by cultured cardiac fibroblasts and the infarcted heart. Am J Physiol 1998;274:H801-9.

68. van Kats JP, Danser AH, van Meegen JR, Sassen LM, Verdouw PD, Schalekamp MA. Angiotensin production by the heart: a quantitative study in pigs with the use of radiolabeled angiotensin infusions. Circulation 1998;98:73-81.

69. Fukushima K, Bravo PE, Higuchi T, Schuleri KH, Lin X, Abraham MR, Xia J, Mathews WB, Dannals RF, Lardo $A C$, Szabo Z, Bengel FM. Molecular hybrid positron emission tomography/computed tomography imaging of cardiac angiotensin II type 1 receptors. J Am Coll Cardiol. 2012 Dec 18;60(24):2527-34.

70. van den Borne S.W.M., Isobe S., Zandbergen H.R. Molecular imaging for efficacy of pharmacologic intervention in myocardial remodeling. J Am Coll Cardiol Img. 2009; 2 (2)187-198.

71. van den Borne S.W., Isobe S., Verjans J.W.; Molecular imaging of interstitial alterations in remodeling myocardium after myocardial infarction. J Am Coll Cardiol.2008;52 (24)2017-2028.

72. Poulsen SH, Host NB, Jensen SE, Egstrup K. Relationship between serum amino-terminal propeptide of type III procollagen and changes of left ventricular function after acute myocardial infarction. Circulation 2000;101:1527-32.

73. Cicoira M, Rossi A, Bonapace S, Zanolla L, Golia G, Franceschini L, Caruso B, Marino PN, Zardini P. Independent and additional prognostic value of aminoterminal propeptide of type III procollagen circulating levels in patients with chronic heart failure. J Card Fail 2004;10 (5):403-11.

74. Radovan J, Vaclav P, Petr W, Jan C, Michal A, Richard P, Martina P.Changes of collagen metabolism predict the left ventricular remodeling after myocardial infarction. Mol Cell Biochem 2006;293:71-8. 


\section{Summary}

Myocardial infarction remains a leading cause of morbidity and mortality worldwide.

The myocardium reacts to an ischemic challenge with a complex series of changes in cellular and extracellular components. The inflammatory component is primarily composed of neutrophil and macrophage infiltration. In particular, the macrophage is a primary responder cell type that is involved in the regulation of post-MI wound healing at multiple levels. According to recent studies, different subsets of macrophages are responsible for these different activities; during the early inflammatory phase (phase 1), proinflammatory macrophages dominate the injury site and phagocytose apoptotic/necrotic myocytes and other debris, whereas during inflammation resolution (phase 2 ), the dominant subsets are the reparative macrophages, which propagate infarct repair.

The first main goal of our research was to study the role of macrophages during myocardial infarction and to establish the correlation between this role and the complex processes leading to collagen deposition, fibrosis, remodeling which may end with irreversible cardiac failure.

Molecular imaging is a highly active research discipline that has seen tremendous growth over the past decade. As it allows for the longitudinal evaluation of biological processes, it is ideally suited for monitoring treatment response. In myocardial infarction and heart failure three biological features represents attractive targets for molecular imaging: 1) apoptosis, a form of programmed cell death which has been shown to play a key role in the process of gradual loss of pump function in heart failure, 2) the reninangiotensin pathway which plays a pivotal role in the development of post-MI heart failure and 3) the biological processes of angiogenesis and fibrogenesis which are considered key processes in myocardial scar formation and post-MI ventricular remodeling.

The second aim of the thesis was to use molecular imaging techniques to gain insight in the (patho)physiological processes following ischemia; focusing on cell death and fibrosis. 
In Chapter 2 we reviewed and discussed the recent progresses made in molecular cardiac imaging, focusing mainly on the achievements made in the integrin alpha $v$ beta 3 (avB3) imaging, apoptosis imaging, and imaging of the activation of the renin-angiotensin system.

From this overview preclinical and clinical data obtained in molecular imaging of adverse left ventricular remodeling, the current outlook for the development of such an imaging technology is promising. For both the imaging of avB3 integrin and imaging of phosphatidylserine expression as a reflection of caspase-3 activation, the preliminary data provide a sufficient basis for the design and execution of novel studies. For imaging of the components of the renin-angiotensin system, the extent of uptake may be insufficient to form a basis for clinical applications. However, before one of these technologies could be adopted as diagnostic tests for routine clinical use, large clinical studies need to be conducted to address key questions. For instance, it is still unknown whether the uptake of avB3 targeting tracers is robust enough to uncover patients that are at the brink of developing adverse remodeling post myocardial infarction. In addition, it remains to be seen whether the extent of the uptake of the tracer can be modulated by treatment with different regimes of cardiac failure treating compounds.

For imaging phosphatidyl serine exposure in the heart using annexin-A5, as a reflection of caspase- 3 activation, the outlook depends largely on the availability of clinically graded annexin-A5 imaging diagnostic kits. With the availability of clinical annexin-A5 imaging kits, studies could be designed to further explore the predictive value of annexin-A5 in patients with failing hearts and to study the effect of therapeutic interventions.

In Chapter 3 we explored the inflammation contributes to the process of ventricular remodeling after acute myocardial injury. To investigate the role of macrophages in the chronic process of cardiac remodeling, they were selectively depleted by intravenous administration of liposomal clodronate in heart failure-prone hypertensive Ren-2 rats from the age of 7 until 13 weeks. Plain liposomes were used for comparison.

Liposomal clodronate treatment reduced the number of blood monocytes and decreased the number of macrophages in the myocardium. Compared to plain liposomes, liposomal clodronate treatment rapidly worsened left ventricular ejection function in hypertensive rats. Liposomal clodronate treated Ren-2 rat hearts showed areas of myocyte loss with abundant inflammatory cell infiltration, predominantly comprising CD4 positive T lymphocytes. Our study showed that lack of macrophages was associated with earlier development of myocardial dysfunction in hypertensive rats. Therefore, macrophages might have a potentially important role in ventricular remodelling in hypertension. Macrophage activation may be important in repair processes and debris clearance and hence depletion of macrophages may not necessarily be an effective strategy in prevention of heart failure. 
In Chapter 4 we used clodronate liposome administration to selectively deplete macrophages early and late after infarction and evaluate the impact on the evolution of left ventricular function.

In clodronate liposome treatment experiments, the functional deterioration was more pronounced when macrophages were present in the initial two weeks after infarction and demonstrated improvement if they were depleted early. The results are intriguing, and it is reasonable to suggest that a reduction in macrophage infiltration early after myocardial infarction may be of some benefit. Although macrophages are necessary for the scavenger function, accompanying cytokine activity may delay the healing process.

In Chapter 5 left ventricular volumes and ejection fraction were quantified based on 7.0 Tesla cine-magnetic resonance imaging in twelve wild-type mice, twelve adipose triglyceride lipase knockout (ATGL ${ }^{-1}$ ) mice (model of impaired cardiac function), and 11 rats in which we induced cardiac ischemia. The left ventricular volumes and function were either assessed with parallel short-axis slices covering the full volume of the left ventricle (gold standard procedure) or with various geometrical models (modified Simpson rule, biplane ellipsoid, hemisphere cylinder, single-plane ellipsoid, and modified Teichholz Formula). Reproducibility of the different models was tested and results were correlated with the gold standard (FV). All models and the FV data set provided reproducible results for the left ventricular volumes and ejection fraction, with interclass correlation coefficients $\geq 0.87$. All models significantly over- or underestimated EF, except for Simpson rule. Good correlation was found for all volumes and ejection fraction for the Simpson rule model compared with the full volume data set. The biplane ellipsoid and hemisphere cylinder models also predicted EF well although proved to be less useful for quantitative analysis. The single-plane ellipsoid and modified Teichholz Formula models correlated poorly with the full volume data set.

For the reduction in acquisition and post-processing time, only the Simpson rule model proved to be a valuable method for calculating left ventricular volumes, stroke volume, and ejection fraction.

In Chapter 6 we tested the hypothesis that that radiolabeled annexin-A5 could be able to identify the regions of myocardial ischemia since apoptotic cell death is characterized by phosphatidylserine externalization on cellmembrane and annexin-A5 avidly binds to phosphatidylserine.

Murine experiments demonstrated that the cardiomyocytes expressed phosphatidylserine on their cell surface after an ischemic insult of $5 \mathrm{~min}$. Phosphatidylserine exposure occurred continuously for at least $6 \mathrm{~h}$ after solitary ischemic insult. Annexin-A5 targeted the exposed phosphatidylserine on cardiomyocytes and it was internalized into cytoplasmic vesicles within 10-30 min. Twenty-four hours after ischemia, cardiomyocytes with internalized annexin-A5 had restored phosphatidylserine asymmetry of the 
sarcolemma, and no detectable phosphatidylserine remained on the cell surface. The pre-administration of a pan-caspase inhibitor prevented phosphatidylserine exposure after ischemia.

Our study demonstrated that after a single episode of ischemia, cardiomyocytes express phosphatidylserine, which is amenable to targeting by annexin-A5 for at least 6 h. Phosphatidylserine exposure is transient and internalized in cytoplasmic vesicles after annexin-A5 binding, indicating the reversibility of the apoptotic process.

In Chapter 7 we examined interstitial alterations during postmyocardial infarction remodeling and assessed the efficacy of antiangiotensin and antimineralocorticoid intervention, alone and in combination by using molecular imaging techniques.

Technetium-99m-labeled Cy5.5 RGD imaging peptide was intravenously administered for gamma imaging after 4 weeks of myocardial infarction in 63 Swiss-Webster mice and in 6 unmanipulated mice. Of 63 animals, 50 were treated with captopril, losartan, spironolactone alone, or in combination, 8 mice received no treatment.

Our findings showed that the quantitative Technetium-99m-labeled Cy5.5 RGD imaging peptide percent injected dose per gram uptake was greatest in the infarct area of untreated control mice and decreased significantly in animals treated with 1 agent. The addition of 2 or 3 agents demonstrated further reduction in tracer uptake proportional to the number of agents employed. The decrease in echocardiographic left ventricular function, strain and rotation parameters, as well as histologically verified deposition of thin collagen fibers, was significantly reduced in treatment groups and correlated with Technetium-99m-labeled Cy5.5 RGD imaging peptide uptake.

Therefore, in our experience, radiolabeled Technetium-99m-labeled Cy5.5 RGD imaging peptide allowed for the evaluation of the efficacy of neurohumoral antagonists after myocardial infarction and reconfirmed the superiority of combination therapy. If proven clinically, molecular imaging of the myocardial healing process may help plan an optimal treatment for patients susceptible to heart failure.

In Chapter 8 we demonstrated the effectiveness of cell death imaging in acute myocardial infarction using a trivalent arsenic peptide, 4-(N-(S-glutathionylacetyl)amino) phenylarsonous acid [GSAO]), which is capable of binding to intracellular dithiol molecules such as HSP9O and filamin-A. Since this peptide is membrane impermeable and dithiol molecules abundantly present intracellularly, we propose that myocardial localization would represent sarcolemmal disruption or necrotic cell death.

In this study we evaluated feasibility of cell death imaging using 111 In-GSAO in mouse and rabbit models of acute myocardial infarction and a mouse model of chronic myocardial infarction. In addition, a subgroup of animals, serial SPECT/CT imaging using 99mTc- annexin-A5 and $111 \mathrm{In}-$ GSAO was performed to determine the relationship between these two modes of cell death in the setting of myocardial ischemia and reperfu- 
sion. Fluorescent GSAO and annexin-A5 were employed in another subgroup of animals for pathological characterization of the mode of cell death.

This study confirmed that the trivalent arsenic group on GSAO is responsible for its targeting characteristics. Our fluorescent experiments showed that GSAO accumulated intracellularly and only occurred in annexin-A5 + cells, thereby supported the notion that necrosis followed apoptotic signalling in the setting of acute myocardial infarction. Hence we can postulate that secondary necrosis is a dominant mode of cell death in the setting of myocardial ischemia and reperfusion.

In our experience, micro-SPECT/CT imaging using 111 In-GSAO can be used to visualize necrotic cell death in acute myocardial infarction whereas the uptake in the heart failure model was too low to allow imaging.

Our studies correlated macrophage infiltration with changes in left ventricular remodeling parameters, confirming the exciting results already seen in the macrophage biology arena revealing avenues for basic science research, in regards to macrophage function in the post-infarction setting specifically and the wound-healing environment. Inhibition studies using anti-inflammatory strategies have taught us the importance of discerning beneficial and detrimental roles for the macrophage post-infarction. The ability to isolate these opposing functions could potentially be used to derive novel therapeutic strategies. Our experiences confirm that high contrast image can be achieved within a specific window in time that will depend on the specifics of the cell death phenomenon under study. Our findings require further larger studies to be confirmed. 



\section{Samenvatting (Summary in Dutch)}

Het myocard infarct (MI) is de belangrijkste doodsoorzaak wereldwijd. Na ischemie doen zich verscheidene veranderingen op cellulair en extracellulair niveau voor. In reactie op de cardiale schade treedt een immuunrespons op; macrofagen en neutrofielen infiltreren het aangedane gebied. De macrofaag speelt een centrale rol in de regulatie van de wondheling na MI. Recente studies hebben aangetoond dat in de verschillende fases van het herstelproces, verschillende subgroepen van macrofagen actief zijn. Tijdens de vroege inflammatie fase (fase 1) zijn pro-inflammatoire macrofagen aanwezig in het beschadigde myocard die apoptotische/necrotische myocyten en overig debris phagocyteren. In de tweede fase zijn met name reparatieve macrofagen die bijdragen aan reparatie van het infarctgebied dominant.

Een van doelstellingen van dit onderzoek was het bestuderen van de rol van macrofagen tijdens en na MI. Hierbij lag de focus op de rol van macrofagen in collageendepositie, fibrose en remodeling; factoren die kunnen bijdragen aan de ontwikkeling van hartfalen.

Moleculaire beeldvorming is een diagnostische techniek die de afgelopen jaren sterk is ontwikkeld. Met moleculaire beeldvorming kunnen biologische processen op moleculair en cellulair geëvalueerd worden. Bij MI en hartfalen zijn er drie biologische processen die middels moleculaire imaging kunnen worden geëvalueerd. 1) apoptose, een vorm van geprogrammeerde celdood die een belangrijke rol vervult in het proces van verlies van pompfunctie bij hartfalen, 2) activatie van het renine-angiotensine systeem dat een belangrijke rol speelt in remodeling en de ontwikkeling van hartfalen na MI en 3) fibrose.

Een tweede doelstelling van dit proefschrift is het toepassen van moleculaire beeldvorming om beter inzicht te krijgen in de (patho)fysiologische processen na het myocardinfarct. De focus lag hierbij op celdood en fibrose.

In hoofdstuk 2 gaven we een overzicht van de recente ontwikkelingen in moleculaire cardiale imaging, waarbij we ons met name richtten op de ontwikkelingen op het vlak 
van moleculaire beeldvorming van integrine alpha v beta 3 (avB3), apoptose en van de activatie van het renine-angiotensine systeem.

Uit onze evaluatie van zowel preklinische als klinische data bleek dat de ontwikkeling van deze beeldvormingstechnologie veelbelovend is. Voor zowel avB3 integrine imaging als imaging van fosfatidylserine expressie ten gevolge van caspase-3 activatie, bleken beschikbare studies reden te geven voor het opzetten van grotere studies. Het is bijvoorbeeld nog onduidelijk of de opname van avB3-gerichte markers voldoende is om patiënten met een hoog risico op het ontwikkelen van remodeling en hartfalen te identificeren. Daarnaast moet nog worden onderzocht of de mate van radiotracer opname gerelateerd kan worden aan de verschillende aspecten in de behandeling van hartfalen. De haalbaarheid van toepassing van annexine-A5 voor beeldvorming van fosfatidylserine expressie (een gevolg van caspase-3 activatie) is met name afhankelijk van de beschikbaarheid van klinische annexine-A5 diagnostische sets. Met behulp van deze klinische annexine-A5 kits zouden studies kunnen worden opgezet waarbij de voorspellende waarde van annexine-A5 in patiënten met hartfalen verder kan worden geëxploreerd alsmede het effect van therapeutische interventie. Voor wat betreft moleculaire beeldvorming van componenten van het renine-angiotensine systeem, blijkt de radiotracer opname voorlopig nog te laag voor het vormen van een basis voor klinische toepassingen.

In Hoofdstuk 3 werd het inflammatieproces dat bijdraagt aan het ontstaan van ventriculaire remodeling na acuut $\mathrm{Ml}$ onderzocht. Om de rol van macrofagen in het chronische proces van ventriculaire remodeling te kunnen onderzoeken, werden deze selectief uitgeschakeld door intraveneuze toediening van liposomaal Clodronate (LC) in hypertensieve Ren-2 ratten tussen de 7 en 13 weken oud. Lege liposomen werden als controle behandeling toegepast. In de hypertensieve ratten behandeld met LC ging de linkerkamerfunctie aanmerkelijk achteruit. Daarnaast lieten de harten van de met LC behandelde ratten gebieden met afname van myocyten en progressieve infiltratie van met name CD4 positieve T lymfocyten zien. Deze studie toonde aan dat een gebrek aan macrofagen gerelateerd was aan vroegere ontwikkeling van myocardiale dysfunctie in hypertensieve ratten; macrofagen spelen dus mogelijk een belangrijke rol in ventriculaire remodeling bij hypertensie. Macrofagen activatie kan van belang zijn voor het herstelproces en het phagocyteren van debris, echter, uitschakeling van macrofagen hoeft niet per definitie een effectieve strategie te zijn in de behandeling van hartfalen.

In hoofdstuk 4 hebben we de rol van macrofagen in de vroege en late fase na het MI onderzocht door macrofaaginfiltratie selectief uit te schakelen middels LC.

Uitschakeling van de macrofaaginfiltratie vanaf de vroege fase na het MI zorgde ervoor dat de linkerkamerfunctie deels behouden bleef.

Deze resultaten zijn fascinerend, en het is reëel om aan te nemen dat een vermindering van macrofagen vroeg na $\mathrm{Ml}$ een voordeel kan zijn. Echter, zoals we in hoofdstuk 3 
hebben laten zien, hebben macrofagen ook gunstige cardiale effecten. Verdere studies zijn daarom nodig om uit te sluiten dat complete uitschakeling van macrofagen na het infarct negatieve gevolgen heeft.

In hoofdstuk 5 werden met behulp van 7.0 Tesla cine-magnetische resonantie imaging (MRI) de linker ventrikel volumes en ejectiefractie bepaald in 12 wild-type muizen, 12 adipeuze triglyceride lipase knock-out $\left(\mathrm{ATGL}^{-/}\right.$) muizen (een model met verminderde cardiale functie) en 11 ratten waarbij cardiale ischemie werd geïnduceerd. De linker ventrikelvolumes en -functies werden gemeten met parallelle korte-as opnamen, waarbij het volledige volume van de linker ventrikel werd geëvalueerd ('gold standard' procedure) of middels verschillende geometrische modellen (gemodificeerde 'Simpson rule,' 'biplane ellipsoid,' 'hemisphere cylinder,' 'monoplane ellipsoid' en gemodificeerde Teichholz formule). De reproduceerbaarheid van de verschillende modellen werd getest en de resultaten werden gecorreleerd met de gold standard (VV). Alle modellen en de FV data set toonden reproduceerbare resultaten voor de linker ventrikel volumes en ejectiefractie met correlatie coëfficiënten van $\geq 0.87$. Alle modellen over- of onderschatten de EF significant, met uitzondering van de Simpson rule. Adequate correlatie werd gevonden voor alle volumes en ejectiefractie voor de Simpson rule vergeleken met de volledige volume data set. De biplane ellipsoid en hemisfeer cylinder modellen voorspelden ook een adequate ejectiefractie hoewel deze methode minder bruikbaar bleek voor kwantitatieve analyse. De monoplane ellipsoid en gemodificeerde Teichholz formule modellen correleerden nauwelijks met de volledige volume dataset. Alleen het Simpson rule model bleek een bruikbare methode voor het berekenen van linker ventrikel volume, slagvolume en ejectiefractie met betrekking tot de procestijd.

In hoofdstuk 6 testten we de hypothese dat gelabeld annexine-A5 de myocardiale ischemische gebieden kon identificeren daar 1) apoptotische cellen phosphatidylserine presenteren op de celmembraan en 2) annexine-A5 eenvoudig bindt aan phosphatidylserine. In een muizenmodel werd aangetoond dat cardiomyocyten phosphatidylserine laten zien op het celoppervlak na een ischemieduur van 5 minuten. Phosphatidylserine bleef continu aanwezig op het celoppervlak voor tenminste 6 uur na ischemie. Annexine-A5 bond zich aan het geëxposeerde phosphatidylserine op cardiomyocyten en werd vervolgens opgenomen in het cytoplasma binnen 10-30 minuten.

Vierentwintig uur na ischemie hadden de cellen de phosphatidylserine asymmetrie hersteld. Er werd geen phosphatidylserine meer aangetroffen op de celmembraan; alle phosphatidylserine was geherinternaliseerd.

De studie toonde aan dat phosphatidylserine wordt gepresenteerd op de celmembraan in cardiomyocyten na een episode van ischemie, en daarmee toegankelijk is voor annexine-A5 tot tenminste 6 uur na ischemie. Phosphatidylserine expressie is tijdelijk en wordt uiteindelijk weer opgenomen in het cytoplasma na binding aan annexine-A5, wat aangeeft dat apoptose reversibiliteit is. 
In Hoofdstuk 7 werd de mate van fibrose na Ml en het effect van anti-angiotensine en anti-aldosteron therapie middels moleculaire beeldvorming onderzocht. Voor deze studie is het myocardinfarct geïnduceerd in 63 muizen; zes ongemanipuleerde muizen werden gebruikt als controles. Van de $63 \mathrm{MI-muizen} \mathrm{werden} \mathrm{er} 50$ behandeld met captopril, losartan en spironolacton, solitair of in combinatie; 8 muizen werden niet behandeld. 4 weken na het MI werd de mate van fibrose geëvalueerd door gamma beeldvorming met technetium-99m-gelabeld Cy5.5 RGD imaging peptide. De radiotracer opname was het meest uitgesproken in het infarctgebied van onbehandelde controle muizen en significant verminderd in de behandelde muizen. Een combinatie van middelen bleek hierbij effectiever dan enkelvoudige therapie. De afname van middels echocardiografie aangetoonde linkerventrikelfunctie en de histologisch geobjectiveerde mate van fibrose, was significant verminderd in de behandelde groepen en correleerden met de opname van de Technetium-99m-gelabelde Cy5.5 RGD imaging peptide. Deze studie bevestigt de superioriteit van combinatietherapie en laat zien dat de effecten van therapieën geëvalueerd kan worden middels technetium-99m-gelabeld Cy5.5 RGD imaging. Indien klinisch bewezen, kan moleculaire beeldvorming van het myocardiale herstelproces helpen een optimaal behandelingstraject voor patiënten met hartfalen samen te stellen.

In hoofdstuk 8 hebben we in diermodellen van $\mathrm{Ml}$ onderzocht of celdood in beeld gebracht kan worden met Technetium-99m-4-(N-(S-glutathionylacetyl)amino) phenylarsonous acid (GSAO). GSAO is een peptide met een trivalente arseengroep dat specifiek bindt aan dithiol moleculen zoals HSP9O en filamine-A. Omdat 1) dithiolmoleculen vrijwel alleen intracellulair voorkomen, 2) deze radiotracer niet getransporteerd wordt over intacte celmembranen, 3) bij necrotische celdood disruptie van de celwand optreedt, was onze hypothese dat deze tracer specifiek zou worden opgenomen in dode cellen met een necrotisch fenotype

In deze studie werd 111 In-GSAO beeldvorming onderzocht in een muis- en konijnmodellen van het acute $\mathrm{Ml}$ aangetoond. Vervolgens werd in een subgroep dieren middels SPECT/CT imaging met zowel 99mTc-annexine-A5 en 111/n-GSAO de relatie tussen apoptose en necrose in het acute $\mathrm{MI}$ aangetoond. Fluorescent gelabeld GSAO en annexine-A5 werden in een andere subgroep toegepast voor immunofluorescentmicroscopische ondersteuning van deze bevindingen. The fluorescentie experimenten lieten zien dat GSAO in de intracellulaire ruimte van cellen wordt opgenomen; dit werd alleen geobserveerd in annexine-A5+ cellen. Dit bevestigt dat necrotische celdood in het acute myocardinfarct volgt op apoptotische celdood; wij postuleren dat deze vorm van secondaire necrose een dominante vorm van celdood is in het acute myocardinfarct. Deze studie bevestigde dat de trivalente arseengroep van GSAO verantwoordelijk is voor de binding aan dode cellen.

Op basis van deze resultaten kan worden geconcludeerd dat 111In-GSAO microSPECT/CT gebruikt kan worden om necrotische celdood in beeld te brengen in acuut MI; de opname in het hartfalenmodel was te laag was om in beeld gebracht te worden. 
We hebben de relatie tussen macrofaaginfiltratie en markers van linker ventrikel remodeling onderzocht. Hierbij werden de baanbrekende resultaten van eerder macrofaagbiologisch onderzoek, die implicaties hadden voor verder fundamenteel onderzoek naar de rol van macrofagen in infarctgenezing bevestigd. Studies waarbij de macrofaaginfiltratie werd geblokkeerd hebben aangetoond dat het belangrijk is de gunstige en ongunstige rollen van macrofagen evalueren. Verdere kennis over deze tegengestelde rollen kan gebruikt worden voor de ontwikkeling van nieuwe therapieën. Onze resultaten laten zien dat hoogcontrast beelden na het infarct gemaakt kunnen worden en dat het tijdsbestek waarbinnen dit mogelijk is afhankelijk is van het type celdood dat in beeld wordt gebracht. 



\section{Valorisatie}

\section{Sociale relevantie}

Myocardinfarct (MI) is de belangrijkste doodsoorzaak wereldwijd. Daarnaast ontwikkelt 40-60\% van de patienten na MI hartfalen. De gevolgen van hartfalen kunnen van grote impact zijn voor de patient en resulteren in hoge kosten binnen de gezondheidszorg. In dit proefschrift worden belangrijke processen, zoals bijvoorbeeld celdood en remodeling, alsmede de componenten welke cruciaal zijn in de ontwikkeling van hartfalen onderzocht.

Naast onderzoek op cellulair niveau naar collageendepositie en fibrose resulterend in remodeling hebben we middels moleculaire imaging getracht beter inzicht te krijgen in de (patho)fysiologische processen na een MI. Hierbij hebben we gebruik gemaakt van verscheidene biomarkers gericht op celdood.

\section{Doelgroepen}

De onderzoeksresultaten van dit proefschrift zijn naast behandelaars, zoals cardiologen en radiologen, ook van betekenis voor (cardiologische) patienten met hartfalen en/of een myocardinfarct. Voor wat betreft de behandelaars kunnen de uitkomsten van dit proefschrift van groot belang zijn met betrekking tot de gepersonaliseerde behandeling voor de patient. Middels de verkregen resultaten kunnen immers de gevolgen van behandeling op een unieke manier voor iedere patient persoonlijk in kaart worden gebracht. De patient heeft op zijn/haar beurt baat bij een adequate en gerichte strategie en behandeling.

\section{Activiteiten en producten}

Verscheidene moleculaire imaging tools, waarvan enkele beschreven in dit proefschrift, kunnen gebruikt worden in de diagnostiek naar het vroegtijdig detecteren van hartfalen en als leidraad dienen voor het behandelingstraject.

Welk moleculair proces het meest geschikt is voor beeldvorming van deze vroege veranderingen hangt af van factoren als beschikbaarheid van het target, gevoeligheid 
van de probe maar ook klinische toepasbaarheid en uiteindelijk beschikbaarheid in de kliniek. Hierbij speelt uiteraard de medische industrie een essentiele rol.

\section{Innovatie, planning, realisatie}

De potentie van moleculaire imaging blijkt uit dit proefschrift zeer waardevol. Immers, de detectie van hartfalen op cellulair niveau alvorens het ziektebeeld door symptomen of anatomische veranderingen tot uiting komt, is essentieel. De diagnostische alsmede de prognostische waarde om de ziekte in de vroege fase te detecteren, kan dankzij het preventieve karakter van moleculaire imaging op zowel maatschappelijk als economisch niveau van ongekende waarde zijn. Daarnaast biedt het de mogelijkheid de therapeutische behandeling te evalueren. Een belangrijke beperkende factor is in dit geval de kosten voor het detecteren van de juiste biomarkers alsmede de aanvullende diagnostiek/beeldvorming welke ervoor noodzakelijk is. Dit proefschrift heeft onder andere de toepasbaarheid van moleculaire imaging aangetoond om een beter beeld te vormen van de verschillende processen welke optreden na MI. 


\section{Dankwoord}

None of us got to where we are on our own. Whether the assistance, guidance or help I received was obvious or subtle, supporting or confronting, scientific or wise, to me acknowledging the impact of someone's help is an essential part of understanding the importance of saying thank you.

So: thank you for those who were there all the time. 



\section{Curriculum vitae}

De auteur van dit proefschrift werd geboren op 17 oktober 1978 te Deventer. Hij volgde het middelbare onderwijs op het Meander College te Zwolle waar hij in 1997 zijn Atheneum diploma haalde. Vanaf 1997 studeerde hij Geneeskunde aan de Universiteit Maastricht. Gedurende de studie was hij werkzaam als student-assistent bij de vakgroep cardiologie alwaar hij zijn eerste co-auteurschappen realiseerde. Het doctoraalexamen werd behaald in 2001. Na zijn co-schappen en behaalde artsexamen in 2004 begon hij als ANIOS bij de vakgroep cardiothoracale chirurgie in het Maastricht Universitair Medisch Centrum. In afwachting van de start van zijn opleiding tot cardiothoracaal chirurg verbleef hij van 2005 tot en met 2007 aan de University of California Irvine waar hij zijn onderzoek met betrekking tot moleculaire cardiologie voortzette. Alvorens hij in 2008 aan zijn vooropleiding Algemene Heelkunde in het Atrium Medisch centrum begon, was hij werkzaam als research assistent aan de Universiteit Maastricht en in het Mount Sinai Medical Center New York. Na het afronden van zijn opleiding tot cardiothoracaal chirurg in 2013 is hij tot op heden werkzaam binnen de staf cardiothoracale chirurgie in het Maastricht Universitair Medisch Centrum. 



\section{Publications}

Tahara N, Zandbergen HR, de Haas HJ, Petrov A, Pandurangi R, Yamaki T, Zhou J, Imaizumi T, Slart RH, Dyszlewski M, Scarabelli T, Kini A, Reutelingsperger C, Narula N, Fuster V, Narula J. Noninvasive Molecular Imaging of Cell Death in Myocardial Infarction using (111)In-GSAO. Sci Rep. 2014 Oct 29;4:6826.

Heemskerk J, Zandbergen HR, Keet SW, Martijnse I, van Montfort G, Peters RJ, Svircevic V, Bouwman RA, Baeten CG, Bouvy ND. Relax, It's Just Laparoscopy! A Prospective Randomized Trial on Heart Rate Variability of the Surgeon in Robot-Assisted versus Conventional Laparoscopic Cholecystectomy. Dig Surg. 2014;31(3):225-32.

van de Weijer T, van Ewijk PA, Zandbergen HR, Slenter JM, Kessels AG, Wildberger JE, Hesselink MK, Schrauwen P, Schrauwen-Hinderling VB, Kooi ME.

Geometrical models for cardiac MRI in rodents: comparison of quantification of left ventricular volumes and function by various geometrical models with a full-volume MRI data set in rodents. Am J Physiol Heart Circ Physiol. 2012 Feb 1;302(3):H709-15.

Kenis $H$, Zandbergen HR, Hofstra L, Petrov AD, Dumont EA, Blankenberg FD, Haider N, Bitsch N, Gijbels M, Verjans JW, Narula N, Narula J, Reutelingsperger CP. Annexin A5 uptake in ischemic myocardium: demonstration of reversible phosphatidylserine externalization and feasibility of radionuclide imaging. J Nucl Med. 2010 Feb;51(2):259-67.

Zandbergen HR, Schellings MW. Molecular imaging of ventricular remodeling. J Nucl Cardiol. 2009 May-Jun;16(3):456-65.

van den Borne SW, Isobe S, Zandbergen HR, Li P, Petrov A, Wong ND, Fujimoto S, Fujimoto A, Lovhaug D, Smits JF, Daemen MJ, Blankesteijn WM, Reutelingsperger C, Zannad F, Narula N, Vannan MA, Pitt B, Hofstra L, Narula J. Molecular imaging for efficacy of pharmacologic intervention in myocardial remodeling. JACC Cardiovasc Imaging. 2009 Feb;2(2):187-98. 
Zandbergen HR, Sharma UC, Gupta S, Verjans JW, van den Borne S, Pokharel S, van Brakel T, Duijvestijn A, van Rooijen N, Maessen JG, Reutelingsperger C, Pinto YM, Narula J, Hofstra L. Macrophage depletion in hypertensive rats accelerates development of cardiomyopathy. J Cardiovasc Pharmacol Ther. 2009 Mar;14(1):68-75.

van den Borne SW, Isobe S, Verjans JW, Petrov A, Lovhaug D, Li P, Zandbergen HR, Ni Y, Frederik P, Zhou J, Arbo B, Rogstad A, Cuthbertson A, Chettibi S, Reutelingsperger C, Blankesteijn WM, Smits JF, Daemen MJ, Zannad F, Vannan MA, Narula N, Pitt B, Hofstra $L$, Narula J. Molecular imaging of interstitial alterations in remodeling myocardium after myocardial infarction. J Am Coll Cardiol. 2008 Dec 9;52(24):2017-28.

Heemskerk J, Zandbergen R, Maessen JG, Greve JW, Bouvy ND. Advantages of advanced laparoscopic systems. Surg Endosc. 2006 May;20(5):730-3.

Palmen M, Daemen MJ, Bronsaer R, Dassen WR, Zandbergen HR, Kockx M, Smits JF, van der Zee $R$, Doevendans PA. Cardiac remodeling after myocardial infarction is impaired in IGF-1 deficient mice. Cardiovasc Res. 2001 Jun;50(3):516-24. 
